Acta Crystallographica Section B

Structural

Science

ISSN 0108-7681

Editor: Carolyn P. Brock

\title{
Structure refinement of $\boldsymbol{H}-\mathrm{Nb}_{2} \mathrm{O}_{5}$
}

K. Kato

This electronic document was scanned from an archival copy of material deposited to accompany a paper published in an IUCr journal. In many cases the only accessible copy was a microfilm of a poor-quality original. 
Structure Refinement of $\mathrm{H}^{-\mathrm{HO}_{2}} \mathrm{O}_{5}$

BY KATSUO KATO

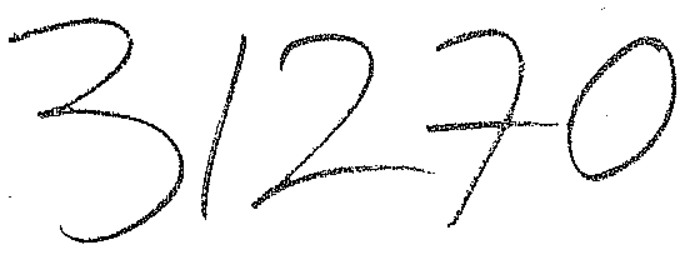

National Institute for Besoarches in Inorganic Materials

Kurakake, Sakura-mura, Niihari-gun, Ibaraki-ken 300-31, Japan

Abstract

The crystal structure of the hifiotemperature modification of ih $\mathrm{O}_{2}\left(\mathrm{i}-\mathrm{Nb}_{2} \mathrm{O}_{5}\right)$ was refined by the least-aquares method based on 70140 observod intensities. The crystal data of $\mathrm{H}-\mathrm{Nb}_{2} \mathrm{O}_{5}$ are $a=21.153(7), b=3.8233(5), c=19.356(5) R, B=119.80(2)^{\circ}$, $z=14, D x=4.548 \mathrm{~g} \mathrm{~cm}^{-3}$. The results agreo generally with those of the previons structure determination [Gatehouse, B. H. \& Wadsley, A. D. (1964). Acta Cryst. 17, 1545-1554] except for the positios of oce tetrahedraily coordinated niobium atom, which is ijistributed equally over $2(i) 0, \pm y, 0$ of $\mathrm{P} 2 / \mathrm{w}$ with $\mathrm{J}=0.2285(3)$. mhe final. $\mathrm{R}$ is 0.052 . $\mathrm{H}-\mathrm{No}_{2} \widehat{0}_{5}$ crystalizizes in the space group p2/ instead of $P 2$ which was assumed in the earlier work. 


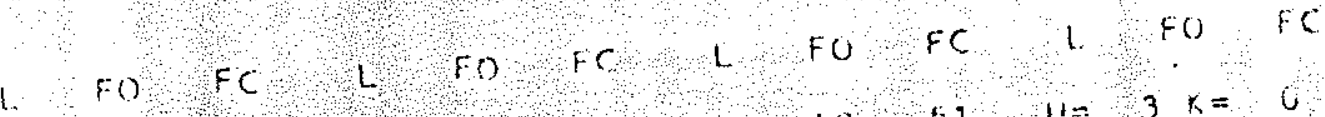

$=\begin{array}{ll}0= \\ 0\end{array}$

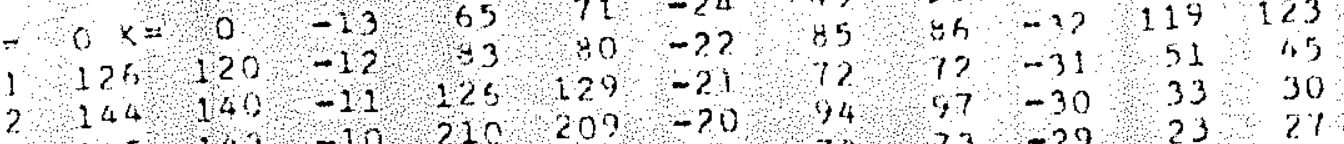

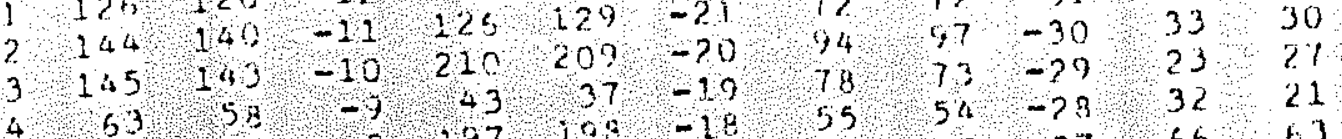

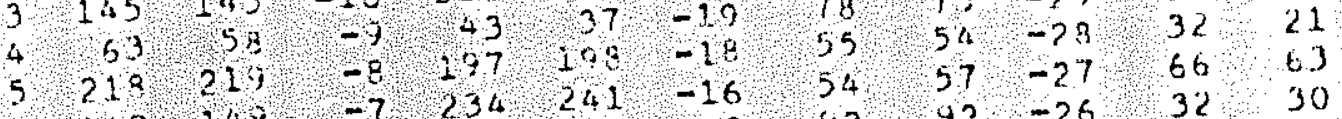

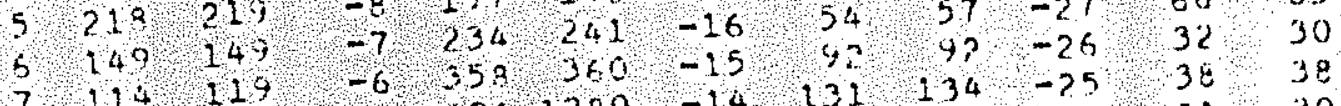

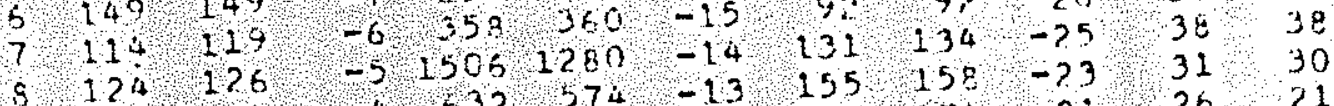

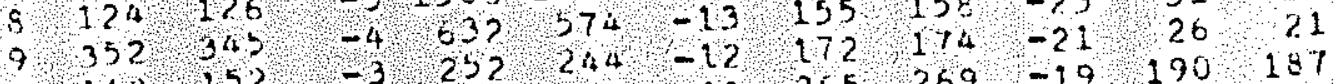

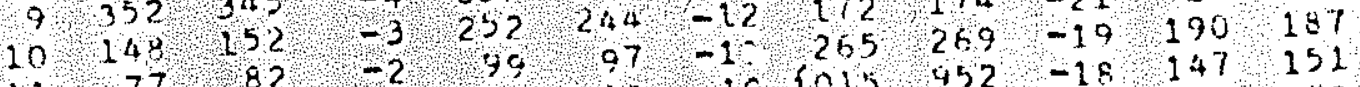

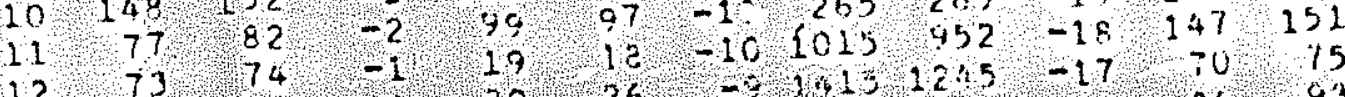

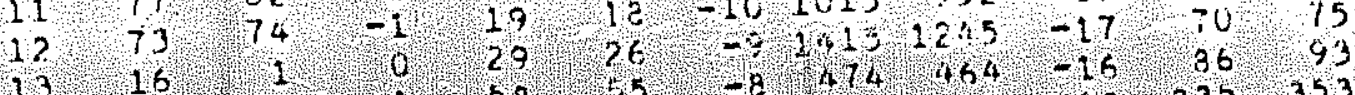

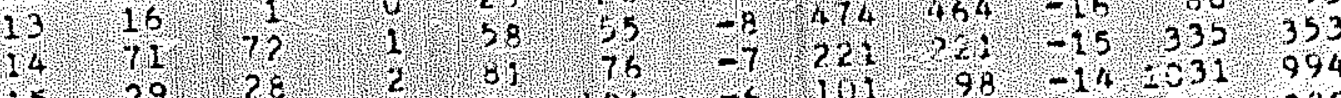

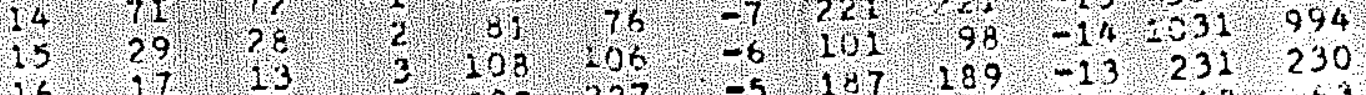

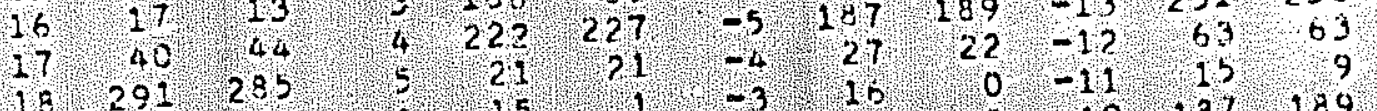

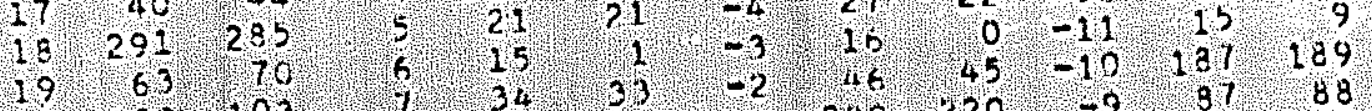

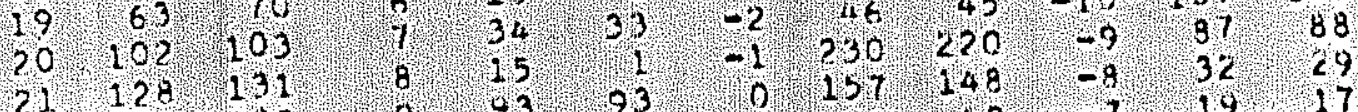

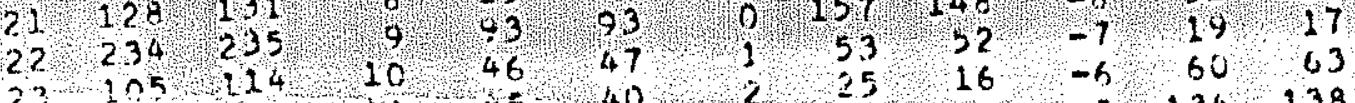

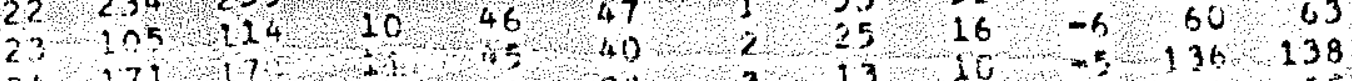

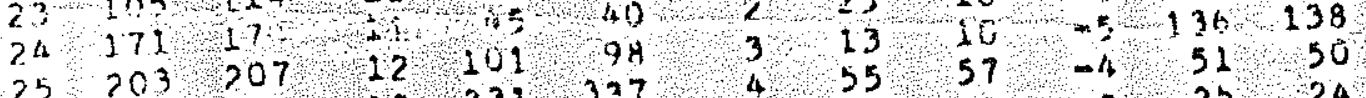

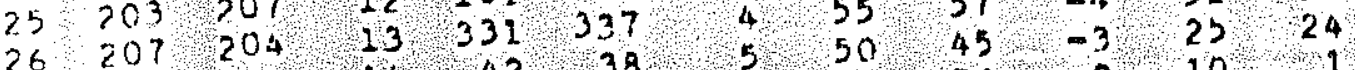

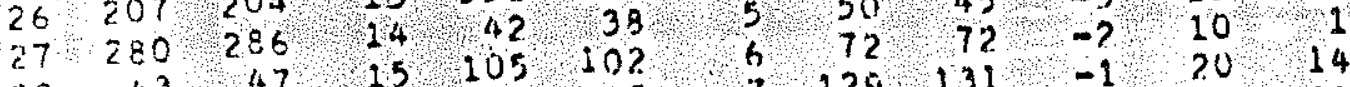

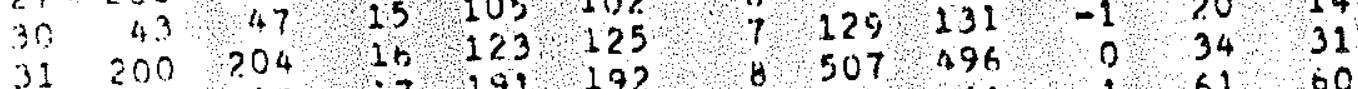

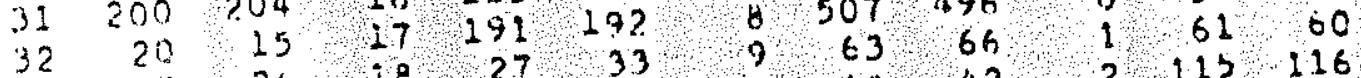

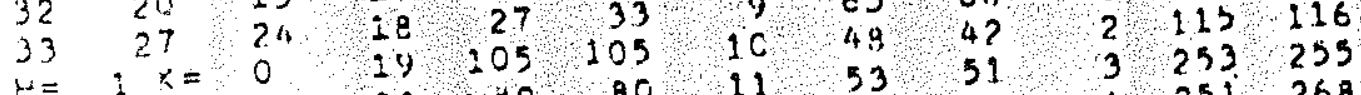

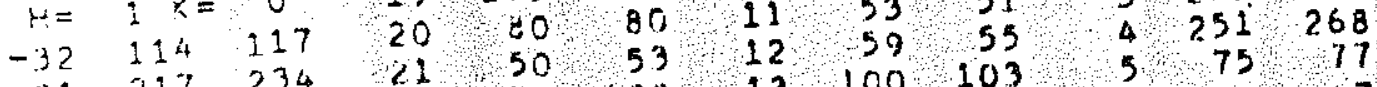

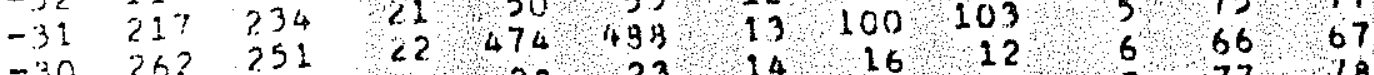

$\begin{array}{llllllll}-30 & 262 & 251 & 2 & 2\end{array}$

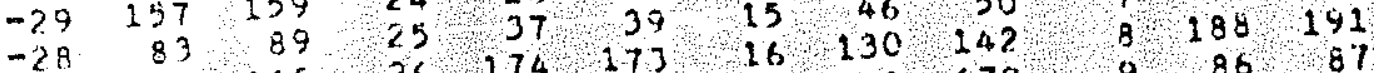

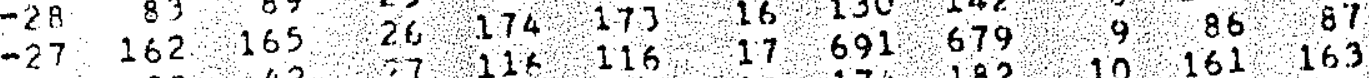

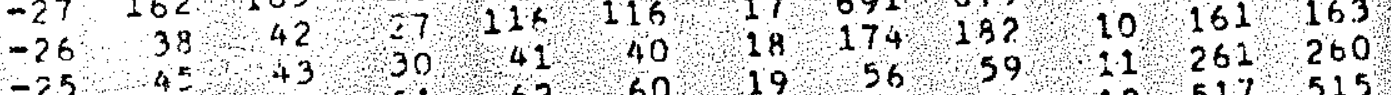

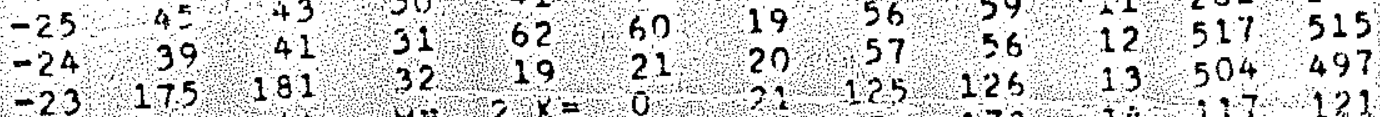

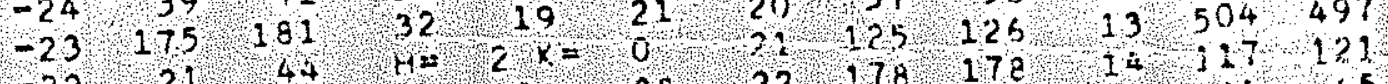

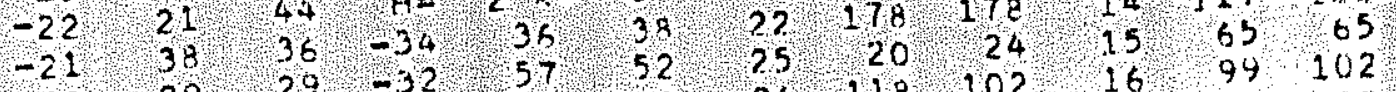

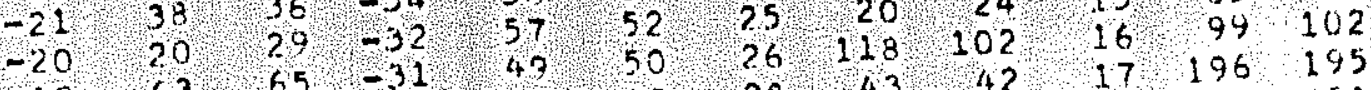

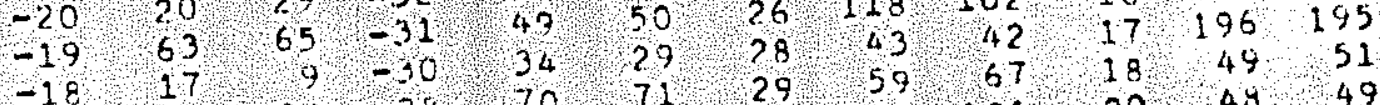

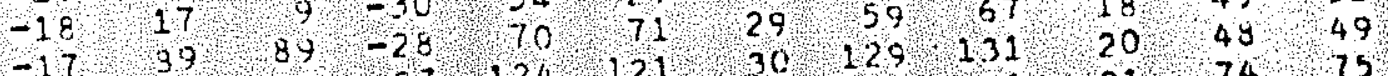

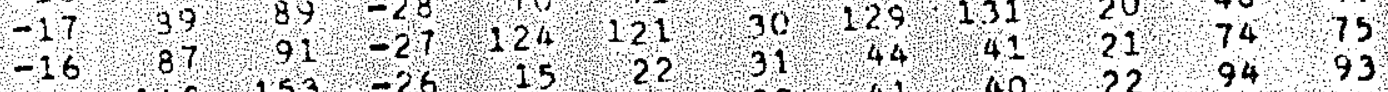

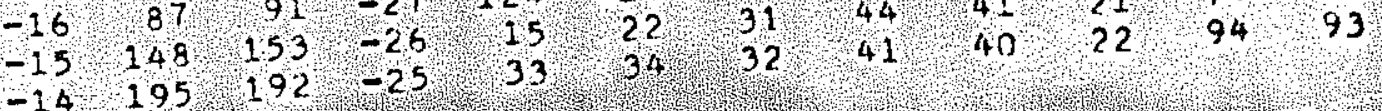


$L \mathrm{FO}$ FC L FO FC L L FO FC L L FO FC

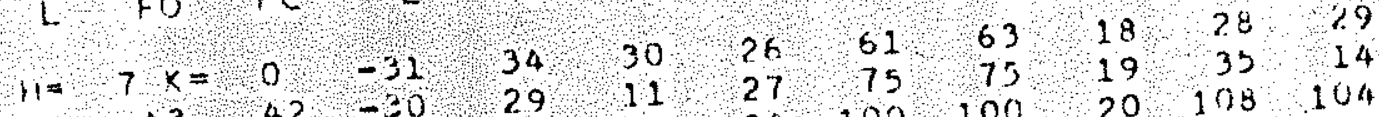

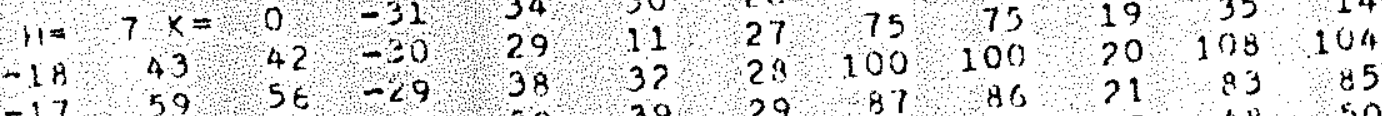

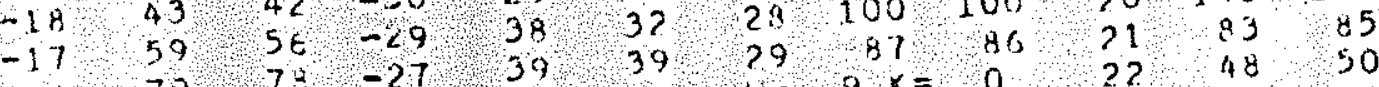

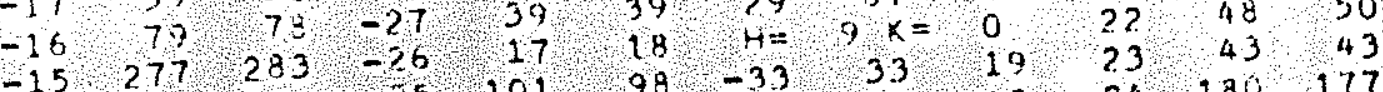

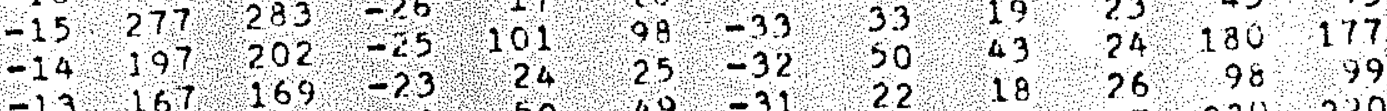

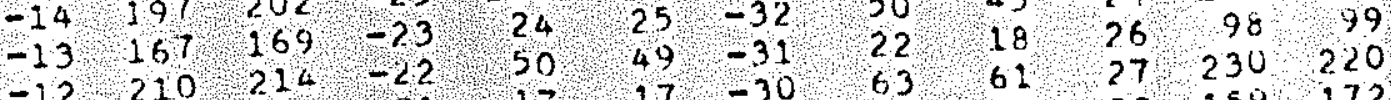

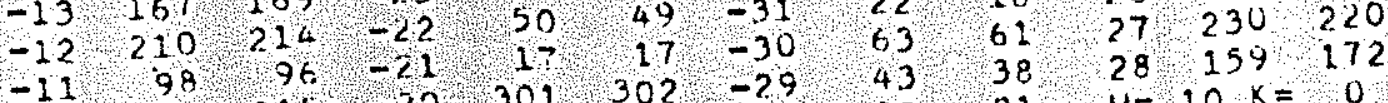

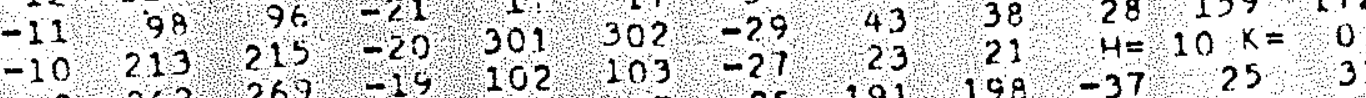

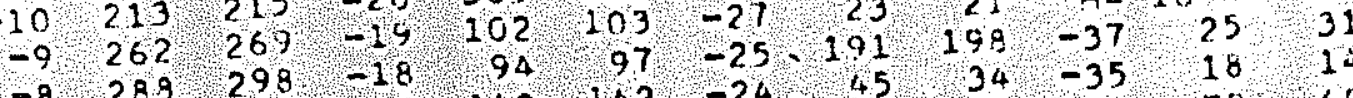

\begin{tabular}{lllllllllllll}
-8 & 289 & 298 & -17 & 140 & 142 & -24 & 45 & 34 & -35 & 18 \\
\hline
\end{tabular}

$\begin{array}{lllllllllll}-6 & 493 & 506 & -76 & 34 & 29 & -22 & 20 & 107 & -33 & 30\end{array}$

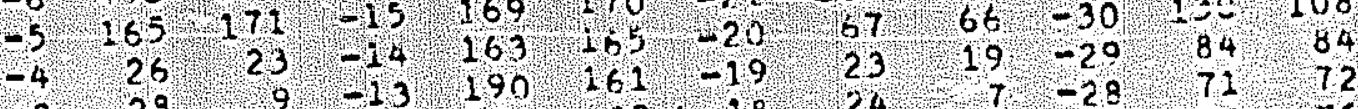

$\begin{array}{lllllllllllllll}-3 & 29 & 9 & -13 & 190 & 161 & -19 & 2 & 7 & -28 & 71 & 72 \\ -2 & 38 & 39 & -12 & 473 & 492 & -18 & 24 & 125 & -27 & 73 & 73\end{array}$

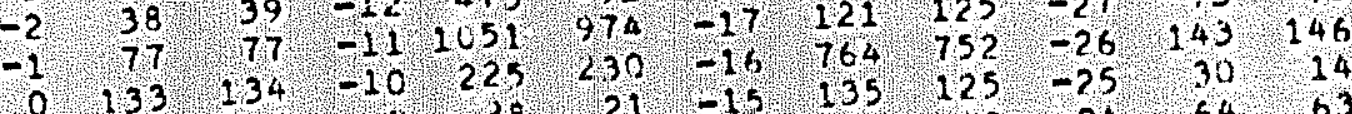

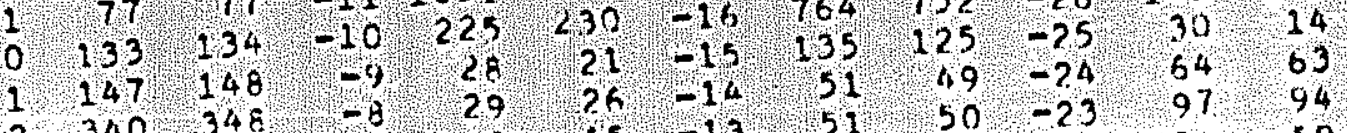

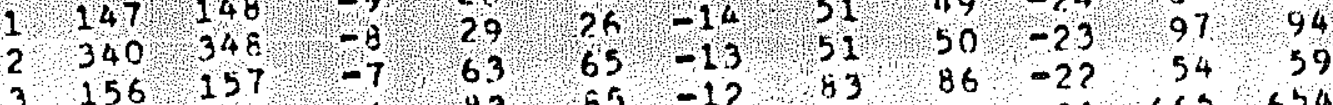

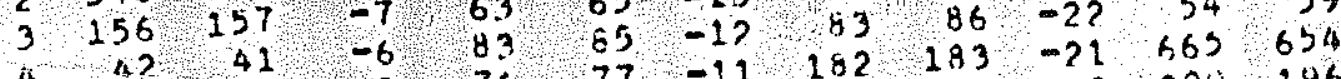

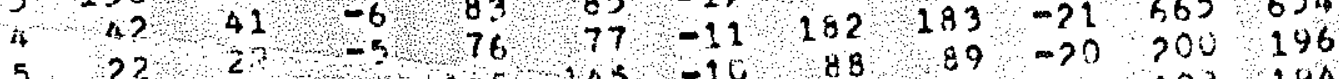

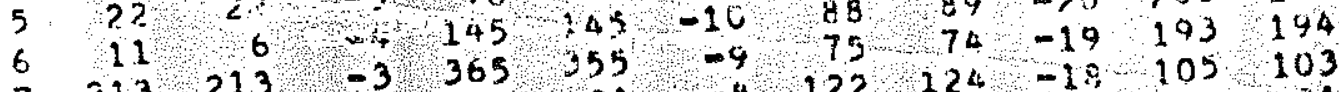

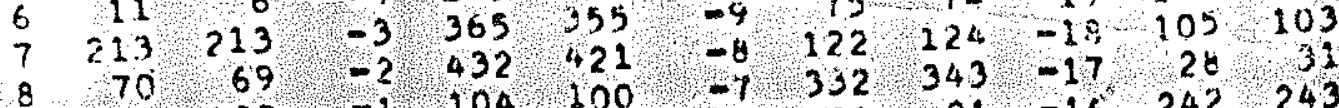

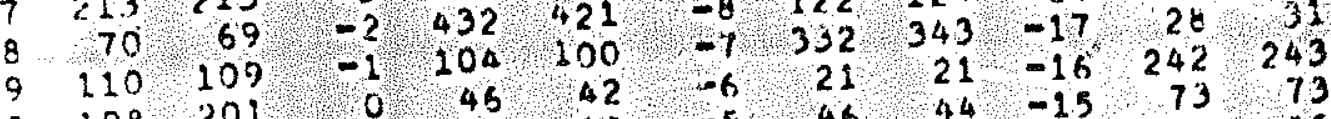

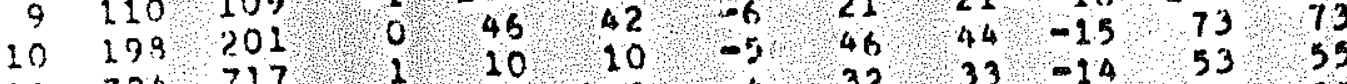

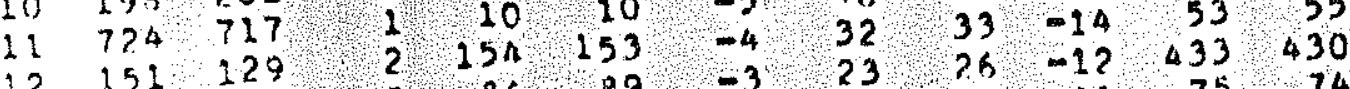

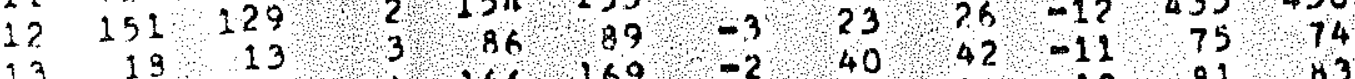

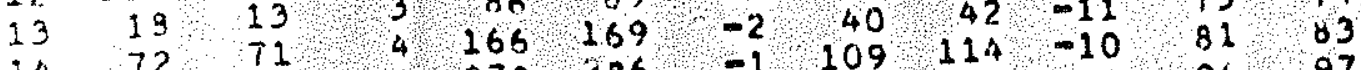

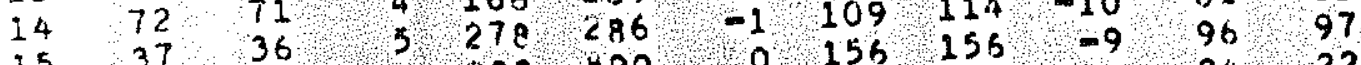

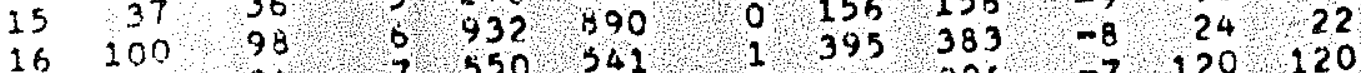

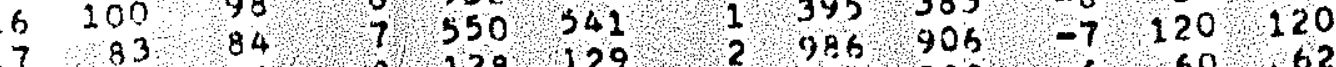

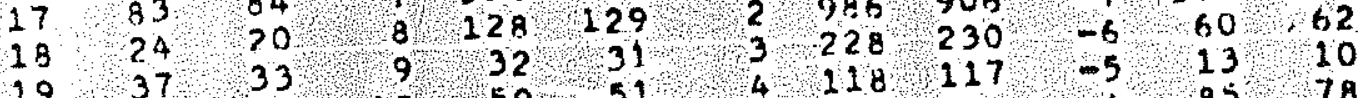

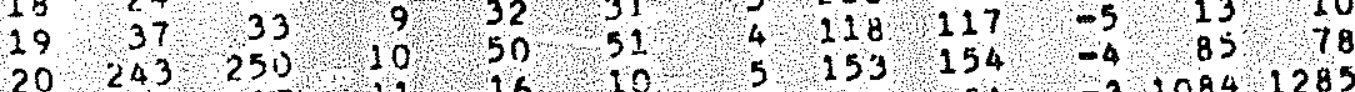

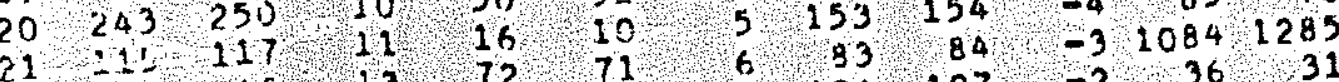

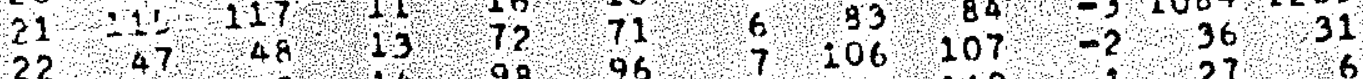

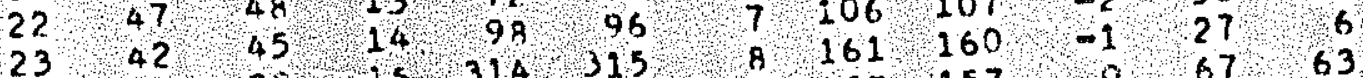

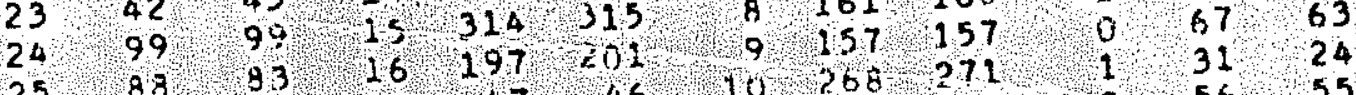

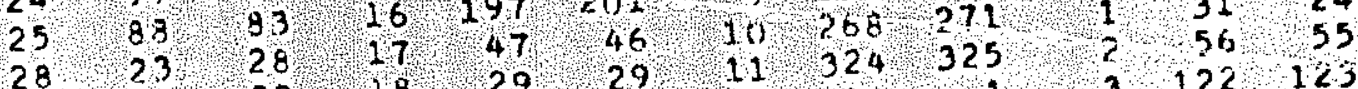

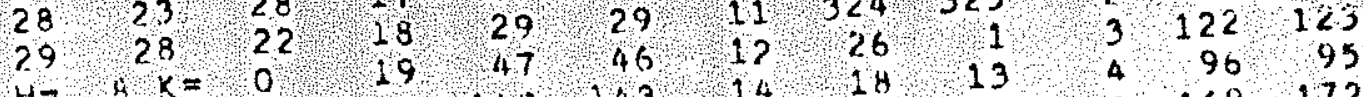

$\begin{array}{llllllll}H= & 8 \\ -35\end{array}$

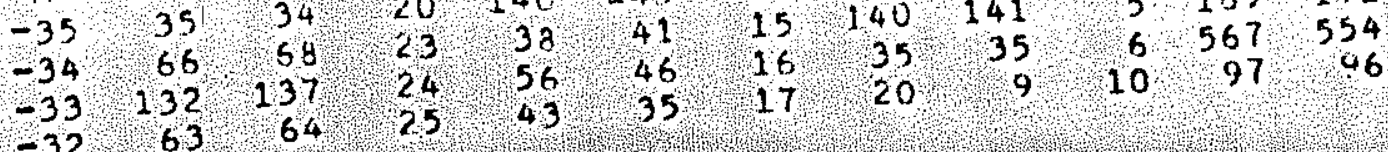




\begin{tabular}{|c|c|c|c|c|c|c|c|c|c|c|c|}
\hline 1 & $\mathrm{FO}$ & $\mathrm{FC}$ & L & $F_{0} O$ & $F C$ & L & $\mathrm{FO}$ & FC & L & $\mathrm{FO}$ & $F C$ \\
\hline$r_{1}=$ & $10 k=$ & 0 & 6 & 137 & 139 & -3 & 241 & 240 & -10 & 202 & 203 \\
\hline ד & 101 & 95 & 7 & 57 & 54 & -1 & 91 & 91 & -9 & 453 & 453 \\
\hline 12 & 35 & 37 & 8 & 74 & 73 & 0 & 86 & 87 & -8 & 371 & 344 \\
\hline & 22 & $2 \%$ & 9 & 90 & 94 & 1 & 150 & 162 & -7 & 19 & 19 \\
\hline 1 & 193 & 190 & 10 & 231 & 224 & 2 & 89 & 90 & -6 & 50 & \\
\hline 1 & 72 & 770 & 11 & 29 & 1 & 3 & 006 & 14 & -5 & 25 & 65 \\
\hline 1 & 20 & 17 & 12 & 52 & 49 & 4 & 117 & 116 & -4 & 96 & 95 \\
\hline & 209 & 209 & 13 & 53 & 55 & 5 & 250 & 244 & -3 & 108 & 10 \\
\hline 2 & 894 & 35 & 14 & 174 & 175 & 6 & 84 & 19 & -2 & 127 & 126 \\
\hline & 141 & 137 & 15 & 1200 & 101 & 7 & 46 & 43 & -1 & 107 & 104 \\
\hline & $12 B$ & 114 & 16 & $3 n$ & 28 & 8 & 32 & 30 & 0 & 125 & 183 \\
\hline & 325 & 327 & 17 & 30 & 8 & 4 & 66 & 66 & 1 & 139 & 136 \\
\hline & 64 & 53 & 18 & 167 & 150 & 10 & 188 & 191 & 3 & 394 & 6 \\
\hline 26 & 45 & 39 & 19 & 504 & 15 & 12 & 45 & 45 & 4 & 96 & \\
\hline 48 & $11 \mathrm{k}=$ & 0 & 20 & 40 & 36 & 12 & 103 & 104 & 5 & 265 & 265 \\
\hline & 50 & 58 & 21 & 32 & 33 & 13 & 203 & 203 & 6 & 104 & 101 \\
\hline & $8 ?$ & 94 & $2 ?$ & 48 & 79 & 14 & 673 & 665 & 7 & 206 & 206 \\
\hline & 94 & 85 & 23 & 43 & 39 & 15 & 103 & 93 & 8 & 290 & 29 \\
\hline & 57 & 50 & 24 & 145 & 144 & 16 & 22 & 3 & 9 & 470 & 486 \\
\hline & 68 & 70 & 25 & $9 ?$ & 95 & 17 & 38 & 34 & 10 & 370 & 37 \\
\hline & 89 & 79 & 26 & 77 & 72 & 18 & 23 & 22 & 11 & 73 & 73 \\
\hline & 26 & 29 & (1) & 107 & 106 & 19 & 158 & 164 & 17 & 31 & \\
\hline & 56 & 53 & $+4=$ & $12 x=$ & 0 & 20 & 82 & 83 & 13 & 34 & 3 \\
\hline & 45 & 43 & -35 & 132 & 130 & 21 & 52 & 29 & 94 & 1.7 & 147 \\
\hline & 353 & 361 & -54 & 24 & 23 & 22 & 58 & 61 & 17 & 25 & 7 \\
\hline & 236 & 245 & -32 & 33 & 30 & 24 & 44 & 43 & 18 & $\begin{array}{l}77 \\
42\end{array}$ & $\begin{array}{l}81 \\
37\end{array}$ \\
\hline & 239 & $<$ & -3 & 307 & 87 & 25 & $13 \mathrm{~K}=$ & $\begin{array}{l}33 \\
0\end{array}$ & 25 & 30 & 37 \\
\hline & $\begin{array}{r}119 \\
272\end{array}$ & 115 & -80 & $\begin{array}{r}405 \\
0,3\end{array}$ & 207 & $\begin{array}{r}H= \\
-37\end{array}$ & 1258 & 45 & $\mathrm{Hu}$ & $14, K=$ & 0 \\
\hline & $\begin{array}{r}272 \\
26\end{array}$ & $\begin{array}{r}269 \\
27\end{array}$ & $\begin{array}{r}-29 \\
-28\end{array}$ & 68 & 68 & -33 & 321 & 324 & -37 & 1929 & 25 \\
\hline-17 & 191 & 187 & -26 & 221 & 224 & -34 & 194 & 177 & -36 & 72 & 73 \\
\hline-16 & 149 & 147 & -25 & 46 & 49 & -33 & 33 & 10 & -35 & 127 & 128 \\
\hline $1=$ & 122 & 12.3 & -24 & 25 & 26 & -31 & 159 & 157 & -34 & 19 & 5 \\
\hline 1 & 75 & 879 & -23 & 34 & 35 & -30 & 107 & 106 & -33 & 24 & 6 \\
\hline 1 & 17 & 12 & -22 & 65 & 63 & -28 & 15 & $4<9$ & -32 & 6.6 & 8 \\
\hline 1 & 214 & 216 & -71 & 269 & 274 & -27 & 24 & 31 & -31 & 145 & 135 \\
\hline & 59 & $\begin{array}{l}58 \\
25\end{array}$ & $\begin{array}{r}-20 \\
-19\end{array}$ & $\begin{array}{r}145 \\
101\end{array}$ & 148 & $-2 h$ & 182 & 179 & $\begin{array}{r}-27 \\
-26\end{array}$ & $\begin{array}{r}140 \\
96\end{array}$ & 140 \\
\hline-9 & $\begin{array}{r}52 \\
46\end{array}$ & $\begin{array}{r}23 \\
47\end{array}$ & $\begin{array}{l}-18 \\
-18\end{array}$ & $\begin{array}{r}201 \\
23\end{array}$ & $\begin{array}{r}100 \\
25\end{array}$ & $\begin{array}{r}-25 \\
-24\end{array}$ & 54 & 53 & -24 & 67 & 604 \\
\hline-8 & 891 & 822 & -17 & 211 & 211 & -23 & 36 & 35 & -22 & 643 & 643 \\
\hline- & 477 & 464 & -15 & 31 & 29 & -22 & 205 & 203 & -21 & 50 & 52 \\
\hline & 341 & 333 & -1, & 23 & 120 & -21 & 67 & 110 & -20 & 50 & \\
\hline & 212 & 10 & -13 & 513 & 309 & -20 & 24 & 27 & -15 & 51 & 54 \\
\hline & 223 & 219 & -12 & 992 & 932 & -19 & 29 & 29 & -15 & 41 & 44 \\
\hline & 178 & 174 & -11 & 443 & 437 & -18 & 147 & 150 & -14 & 143 & 144 \\
\hline & 108 & 103 & -10 & 263 & 266 & -17 & 726 & 710 & -23 & 584 & \\
\hline & 140 & 141 & 1 & 261 & 261 & -16 & 2,2 & 243 & -12 & 20 & \\
\hline 0 & 136 & 136 & $1-8$ & 243 & 242 & -15 & 109 & 109 & -11 & 102 & 103 \\
\hline 1 & 118 & 694 & (2) & 203 & $20 ?$ & -1, & 113 & 120 & -10 & 57 & \\
\hline 2 & 38 & 438 & r -6 & 27.5 & 279 & -13 & 99 & 117 & -9 & 20 & 5 \\
\hline 3 & 49 & 50 & -5 & 314 & 307 & -12 & 86 & 86 & -8 & 80 & 81 \\
\hline 4 & 70 & 69 & -4 & 172 & 755 & -11 & 169 & 171 & -7 & 67 & \\
\hline
\end{tabular}




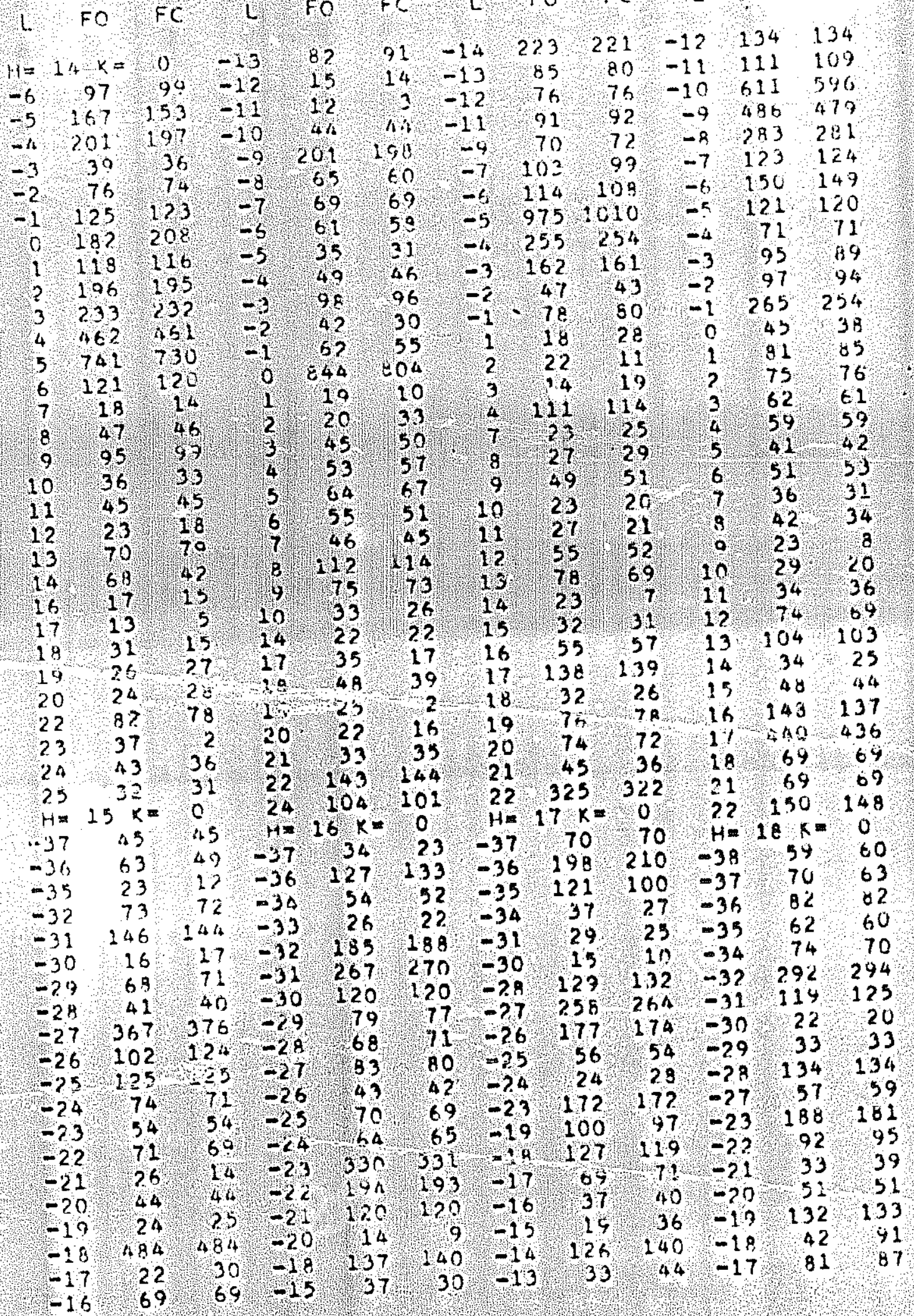




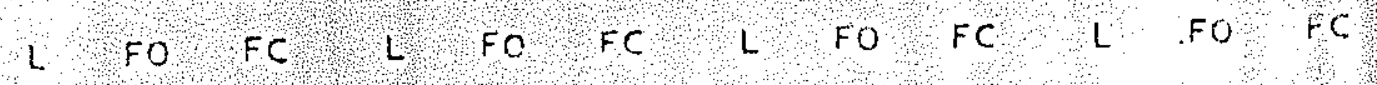

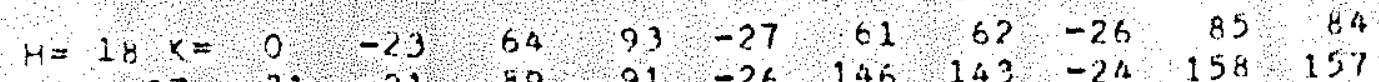

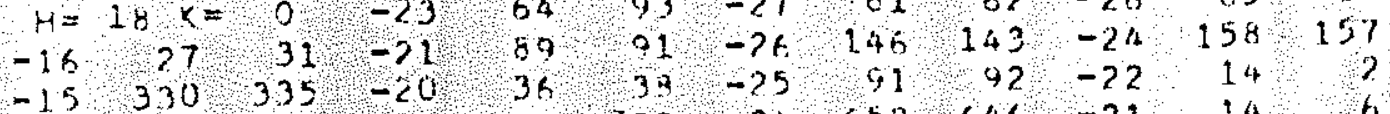

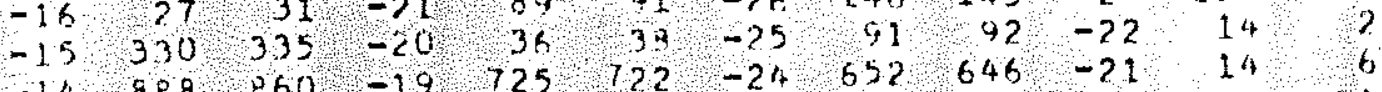

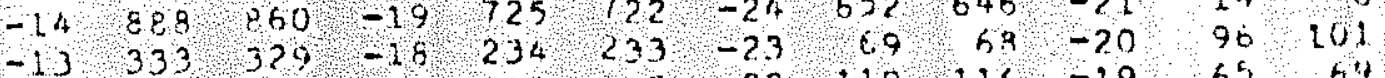

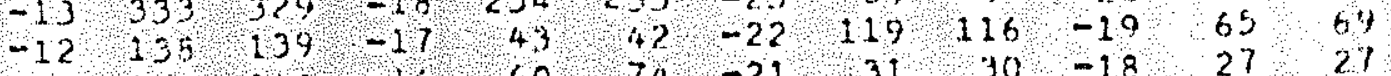

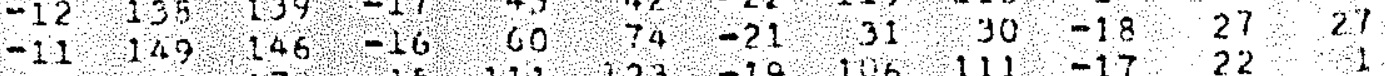

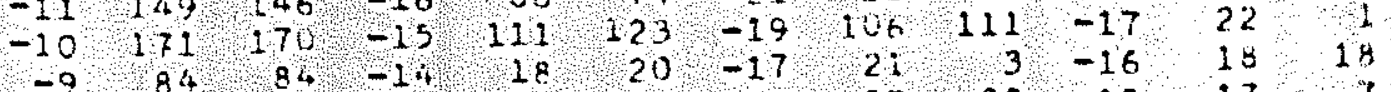

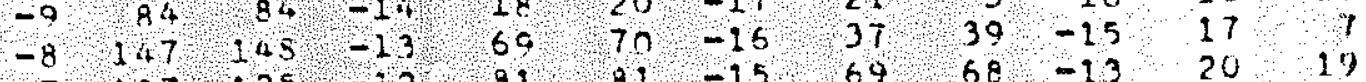

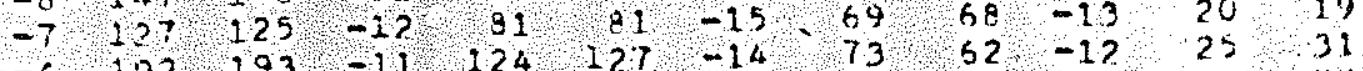

$\begin{array}{lllllllllll}-6 & 172 & 193 & -11 & 124 & 179 & -13 & 61 & 62 & -11 & 92\end{array}$

$\begin{array}{llllllllllllll}-4 & 93 & 90 & -9 & 59 & 52 & -12 & 21 & 9 & -10 & 26 & 16\end{array}$

$\begin{array}{llllllllllll}-3 & 13 & 132 & -8 & 70 & 66 & -10 & 19 & 20 & -9 & 48 & 41 \\ -2 & 110 & 109 & -7 & 76 & 73 & -9 & 32 & 33 & -8 & 26 & 23\end{array}$

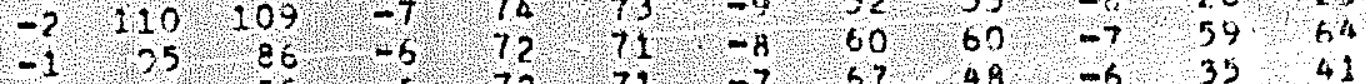

$\begin{array}{lllllllllllll}0 & 54 & 55 & -5 & 72 & 71 & -7 & 5 & 48 & -6 & 35 & 41\end{array}$

$\begin{array}{llllllllllll}1 & 46 & 52 & -4 & 62 & 6 ? & -6 & 29 & 229 & -5 & 38 & 15\end{array}$

$\begin{array}{lllllllllllll}2 & 3 & 43 & -3 & 49 & 46 & -5 & 95 & 96 & -3 & 9 & 0\end{array}$

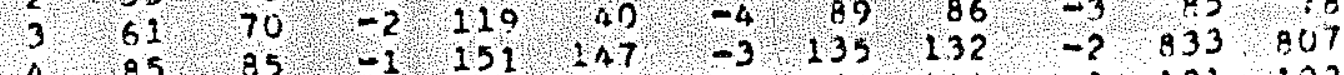

$\begin{array}{llllllllllll}4 & 85 & 85 & -1 & 151 & 147 & -3 & 13 & 132 & -2 & 121 & 122\end{array}$

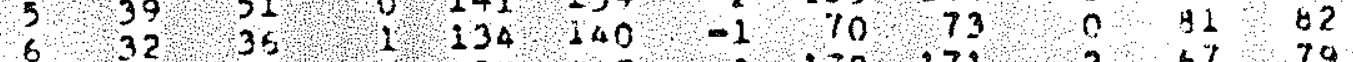

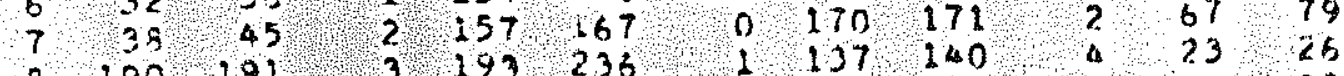

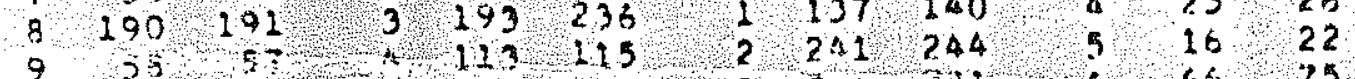

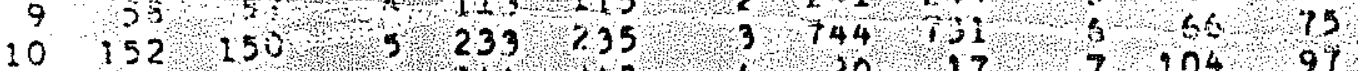

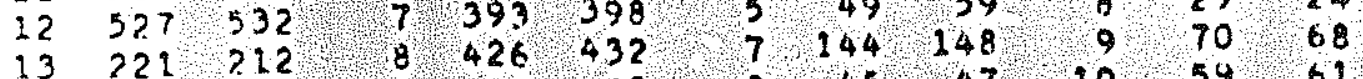

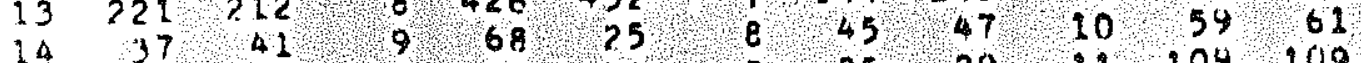

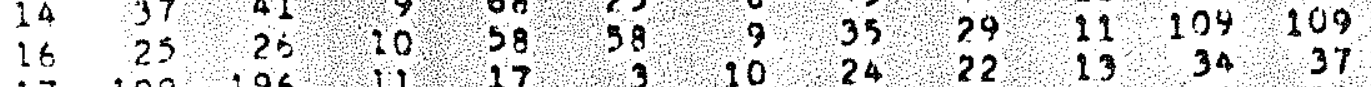

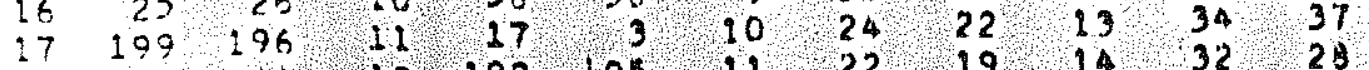

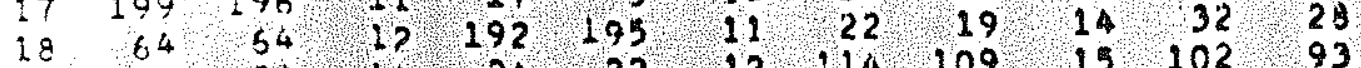

$\begin{array}{llllllllllll}19 & 32 & 30 & 16 & 24 & 22 & 12 & 1 & 14 & 109 & 15 & 102 \\ 2 & 96 & 99 & 15 & 64 & 68 & 13 & 40 & 36 & 16 & 156 & 161\end{array}$

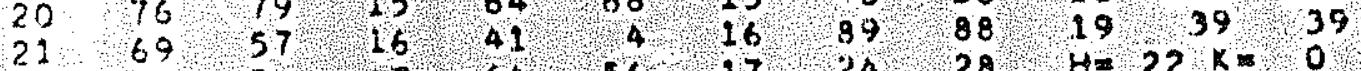

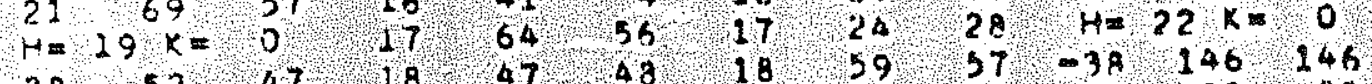

$\begin{array}{lllllllllllll}-38 & 52 & 47 & 18 & 47 & 40 & 18 & 59 & 37 & -39 & 35 & 35\end{array}$

$\begin{array}{llllllllllll}-37 & 213 & 213 & 19 & 22 & 21 & 19 & 89 & 82 & -37 & 36\end{array}$

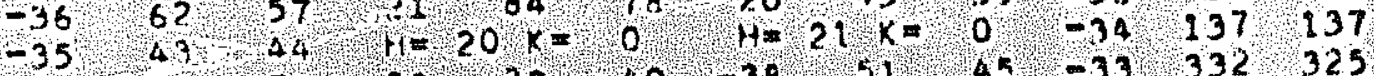

\begin{tabular}{lllllllllllll}
-33 & 77 & 76 & -36 & 30 & 10 & -38 & 51 & 45 & -33 & 332 & 325 \\
\hline & 7, & 70 & -38 & 77 & 78 & -12 & 184 & 191
\end{tabular}

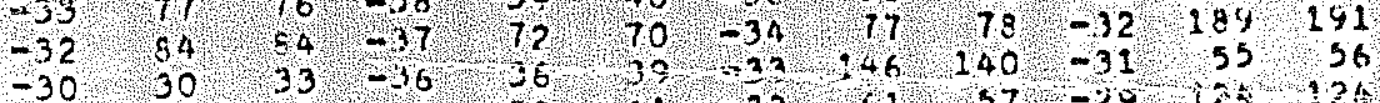

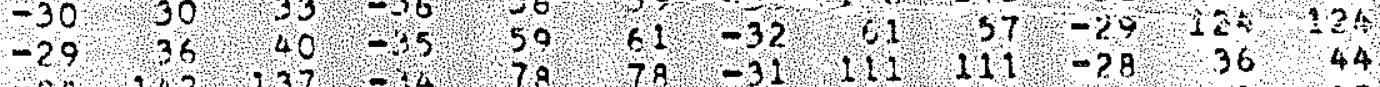

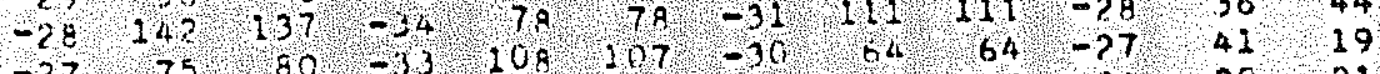

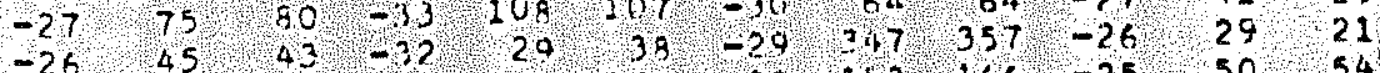

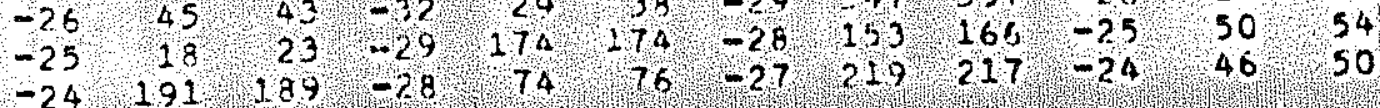




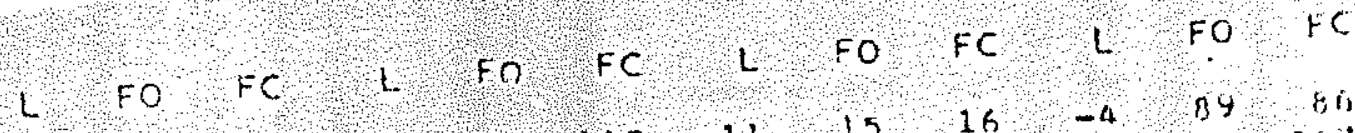

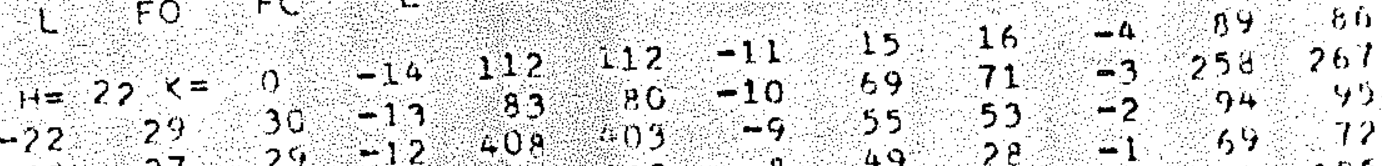

-20
-1

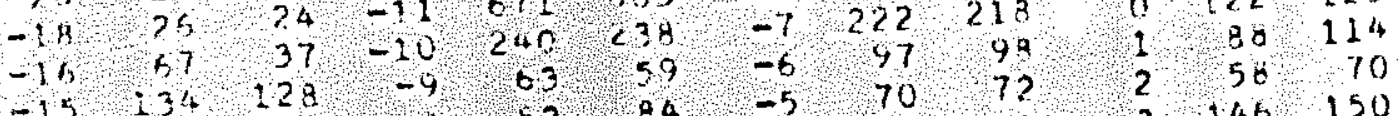

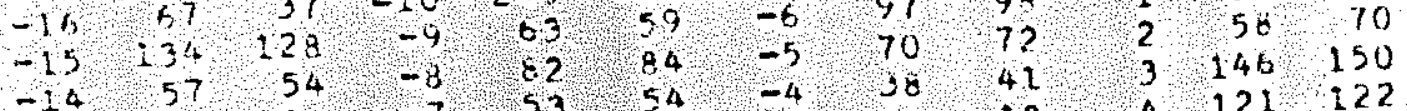

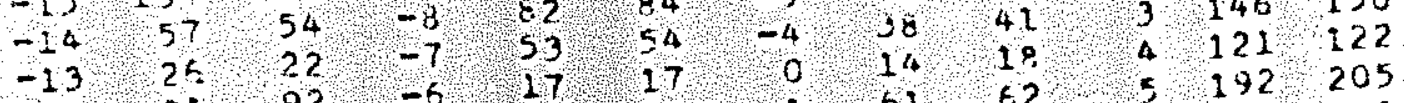

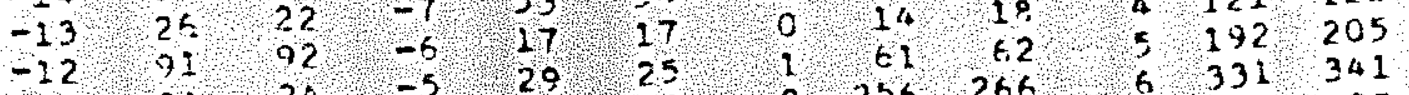

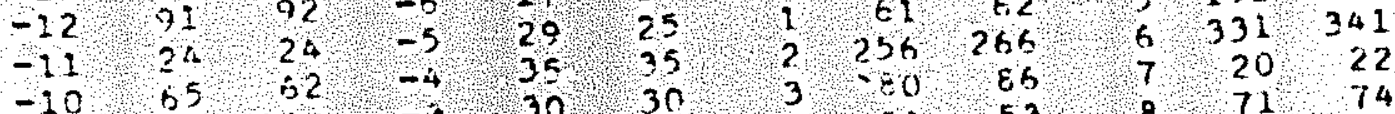

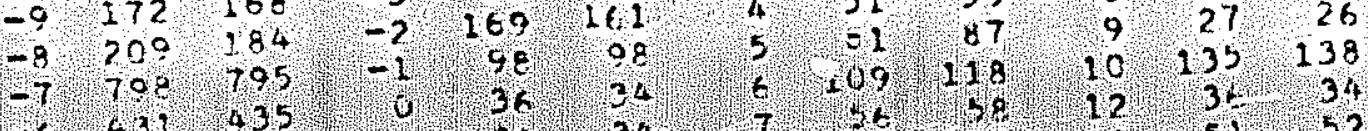

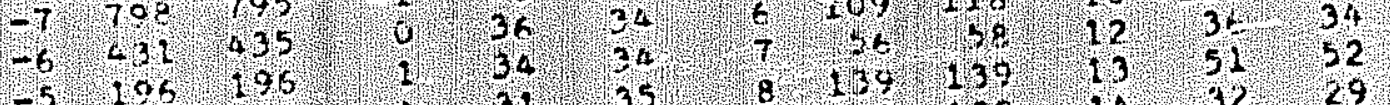

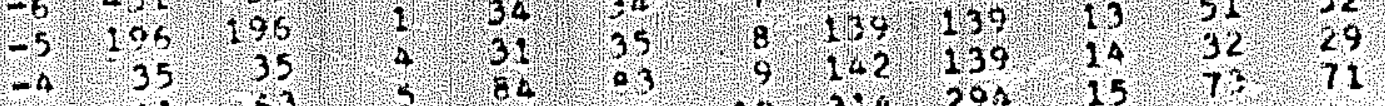

$\begin{array}{llllllllllllllll}-3 & 3 & 6 & 6 & 5 & 84 & 03 & 91\end{array}$

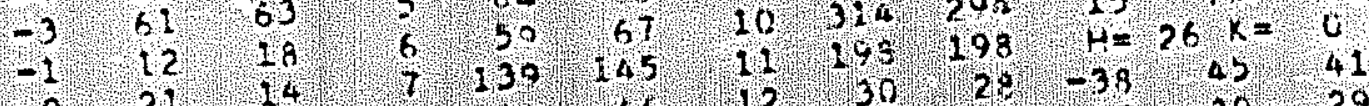

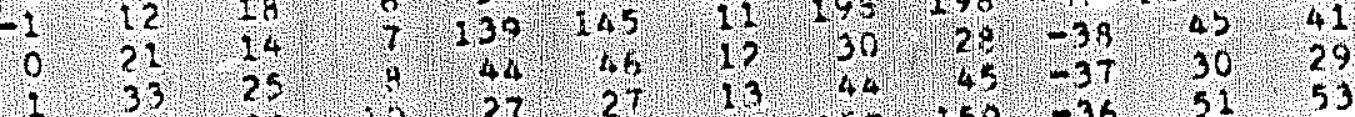

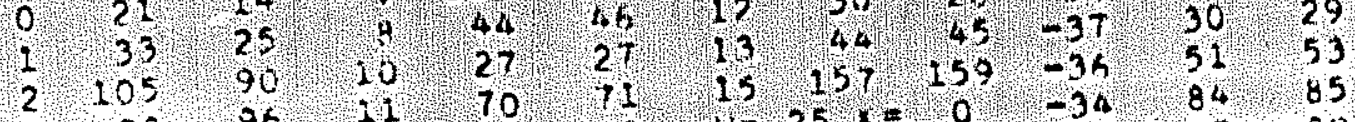

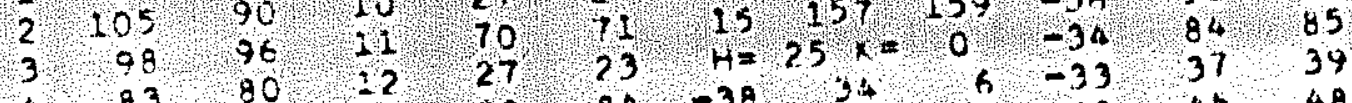

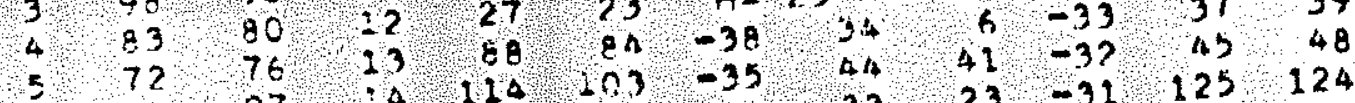

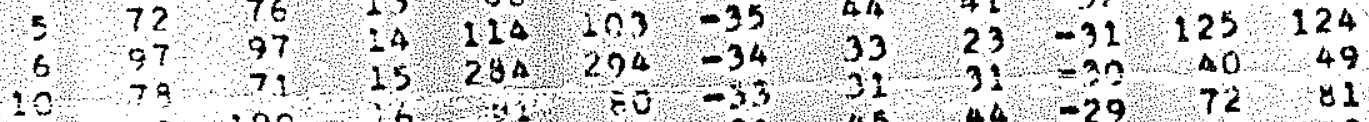

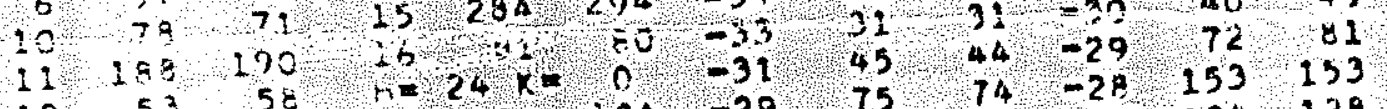

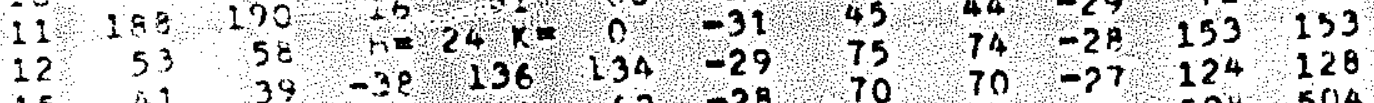

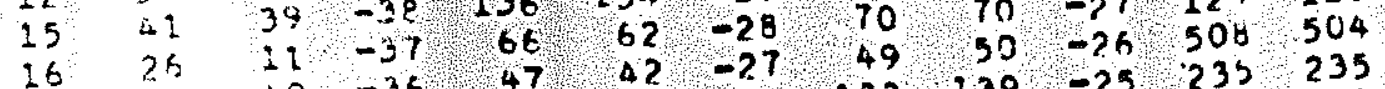

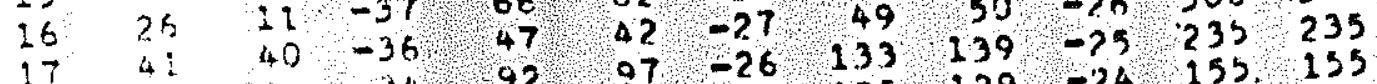

$17 \quad 23 r=0 \quad-34 \quad 92 \quad 07,-26 \quad 125$ 129 $\quad-24,155,135$

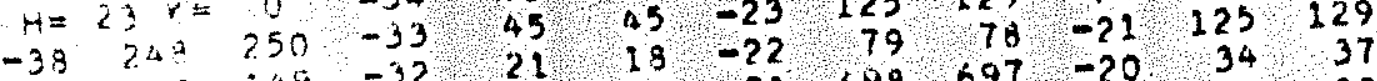

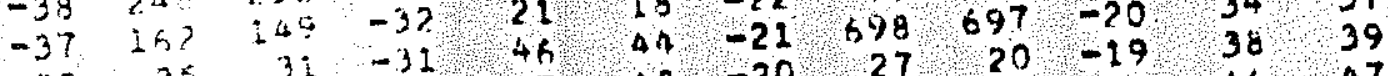

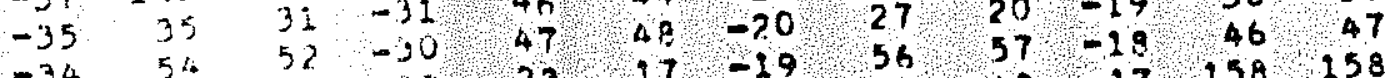

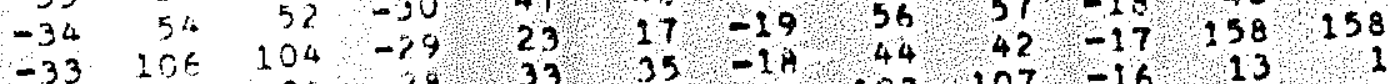

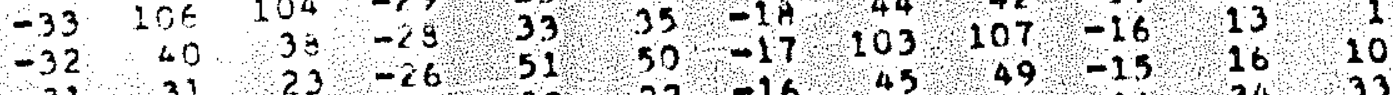

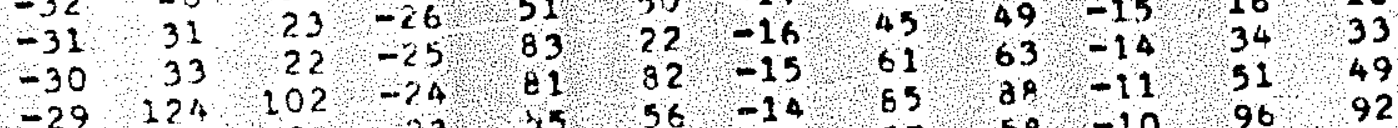

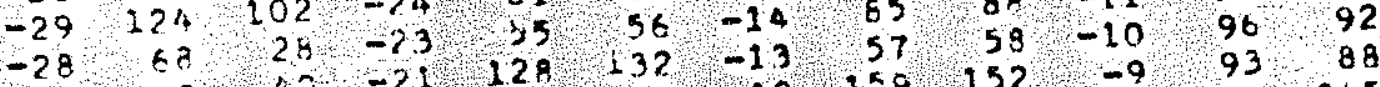

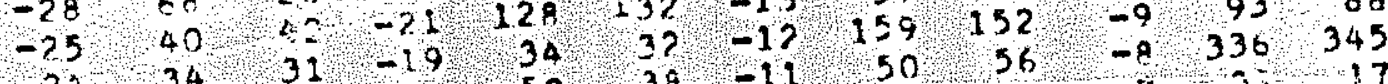

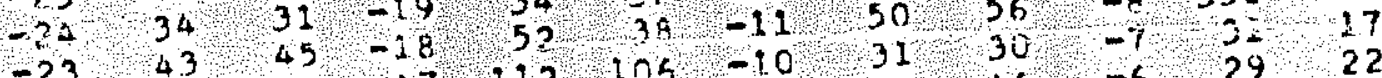

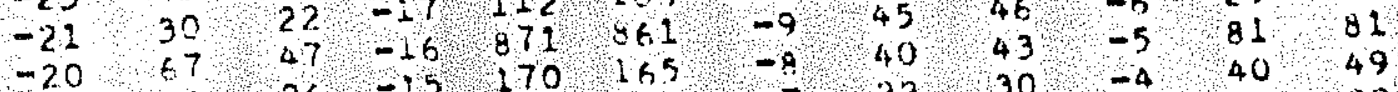

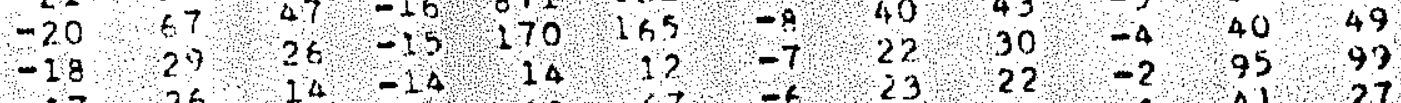

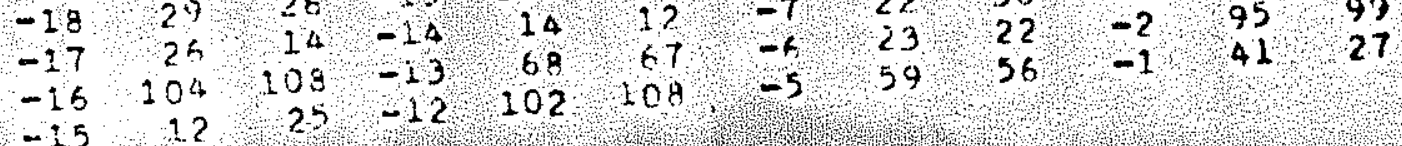




\begin{tabular}{|c|c|c|c|c|c|c|c|c|c|c|c|}
\hline$L$ & FO & $F C$ & L & $F O$ & $F C$ & 1 & 50 & FC & L. & Fu & \\
\hline$H=$ & $26 \quad k=$ & 0 & $\epsilon$ & 89 & 75 & -34 & 57 & $5 t_{*}$ & -15 & 74 & \\
\hline 0 & 65 & 59 & 7 & 79 & 94 & -32 & 24 & 21 & -14 & 6.4 & \\
\hline 1 & 472 & 476 & 8 & 56 & 59 & -31 & 41 & 24 & $-1 ?$ & 1,4 & \\
\hline 2 & 81 & 79 & 9 & 93 & 00 & -30 & $3 t$ & 37 & -11. & 44 & \\
\hline 3 & 9 & 100 & 10 & 55 & 5,5 & $-? 0$ & 34 & 1 & 10 & 31 & \\
\hline 4 & 25 & 26 & 11 & 34 & 35 & -27 & $\angle 0$ &. & -9 & 234 & \\
\hline 5 & 39 & 96 & 13 & 19 & 80 & -26 & 84 & e & -8 & 31 & \\
\hline 6 & 3 & 50 & $r=$ & $2 E r=$ & 0 & -25 & 50 & 5 & -7 & 16 & \\
\hline 8 & 21 & 28 & -37 & $|27|$ & 24 & -24 & 14 & 15 & -5 & 44 & \\
\hline 10 & 114 & $11 \%$ & -16 & 15 & 34 & -23 & & $?$ & -3 & 29 & \\
\hline 12 & 35 & 23 & -35 & 56 ? & 356 & -22 & & 17 & -1 & & \\
\hline 13 & 45 & 4 & -34 & 117 & & -21 & 118 & 121 & 0 & 490 & \\
\hline 14 & 126 & 172 & -31 & $10 ?$ & 207 & -99 & 40 & 3 & 1 & 97 & \\
\hline$r=$ & $27 k=$ & 100 & -30 & 108 & 114 & 18 & 22 & 2 & 2 & 24 & \\
\hline 38 & 29 & 2 & -29 & 55 & 57 & -14 & 13 & 6 ? & & 89 & \\
\hline & 2 & 4 & -28 & 30 & 32 & 213 & 30 & Wis & 2 & 17 & \\
\hline 35 & 01 & 3 & -27 & 30 & 36 & -14 & 141 & 143 & 5 & 43 & \\
\hline 34 & 60 & 70 & -26 & 42 & 76 & -13 & $52 \%$ & 537 & $\theta$ & 115 & \\
\hline 33 & 105 & 108 & -25 & 4. & 48 & -12 & 97 & 9 & 7 & & \\
\hline 32 & 153 & 4 & -24 & 10 & 21 & -11 & 1 & & $a$ & 125 & \\
\hline 31 & 231 & 276 & -23 & 3 & 51 & -20 & 28 & 20 & 9 & 70 & \\
\hline 30 & 323 & 312 & -21 & 76 & 74 & -5 & 13 & 21 & $H=$ & $39 k=$ & \\
\hline 29 & 179 & 202 & -20 & 71 & 73 & -6 & 28 & $3 n$ & -36 & 196 & \\
\hline 98 & 41 & 144 & -20 & 34 & 26 & -5 & 22 & 10 & -33 & 26 & \\
\hline 26 & 165 & 170 & -18 & 110 & 125 & -4 & 206. & 212 & -37 & 63 & \\
\hline 25 & 46 & 51 & -17 & 205 & 218 & -3 & $4 b$ & 47 & -31 & 46 & \\
\hline 4 & 37 & 35 & -15 & 118 & 128 & 10 & 72 & 40 & $-3 n$ & 152 & \\
\hline 23 & 15 & 7 & -16 & 440 & 3 & $?$ & 26 & 90 & -29 & 86 & \\
\hline 22 & $6 \pi$ & 52 & -14 & a? & 8 & 3 & & 106 & -70 & 115 & \\
\hline $2 \pm$ & 41 & 1 & -13 & 5. & 49 & 4 & & 173 & -27 & 37 & \\
\hline 20 & 31 & 3 & -12 & 40 & 39 & 3 & 275 & $28:$ & -26 & 5 & \\
\hline 19 & ?. 1 & $?$ & -11 & 131 & 132 & 6 & 08 & 70 & -25 & 22 & \\
\hline 17 & 40 & 4 & -10 & 100 & ic & & 49 & 4 & -24 & 91 & \\
\hline 15 & 4 & 4 & - & $37 n$ & 37 & 12 & 607 & 63 & -23 & 410 & \\
\hline 15 & 35 & $\Leftrightarrow$ & -8 & 380 & 399 & $y=$ & $30 \mathrm{~K}=$ & 0 & -22 & 12 & \\
\hline 14 & 70 & 7 & $=7$ & 119 & 12 & -36 & 26 & 2 & -21 & 74 & \\
\hline 13 & 171 & 17 & -4 & 3 & 4 & -34 & 47 & 41 & -20 & 2.4 & \\
\hline 12 & 42 & 5 & -1 & 3 & ? 4 & -33 & 62 & 53 & -19 & 75 & \\
\hline & 67 & 72 & 10 & .94 & 99 & -31 & $<5$ & 6 & -1 & & \\
\hline 10 & 19 & 7 & 1 & 145 & 442 & -30 & 21 & 21 & -17 & 60 & \\
\hline- & 55 & $5:$ & 2 & 77 & 8 & -29 & 24 & $?$ & -16 & 1 & \\
\hline-8 & 37 & 4. & 3 & 4 & 5 & -98 & 17 & 17 & -15 & 57 & \\
\hline-6 & 93 & 92 & 4 & 50 & 5 & -27 & 171 & 17 & -14 & 216 & \\
\hline & 99 & 16 & 5 & 77 & 8. & -25 & 17 & 7 & -1 & 32 & \\
\hline- & 494 & 50 & 7 & 5 & 5 & -7 & 28 & 34 & -11 & 42 & \\
\hline- & 200 & 20 & 8 & $\div 6$ & 9 & 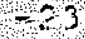 & 21 & & -10 & 5 & \\
\hline & 29 & 19 & 9 & 297 & 29 & -2 & 36 & 43 & $2-9$ & 49 & \\
\hline 0 & 38 & 4 & 10 & 134 & 137 & -21 & 29 & 2 & $-R$ & 42 & \\
\hline & $4 \pi$ & 6. & 11 & 30 & 44 & -20 & 97 & 93 & -7 & 113 & \\
\hline 3 & 28 & 2 & $\sqrt{1}=$ & $29 k=$ & 0 & -19 & 41 & 32 & -5 & 122 & \\
\hline 4 & 47 & 4 & -37 & 464 & 6 & -18 & 550 & 552 & -5 & 525 & \\
\hline 5 & $1<3$ & 123 & -35 & 172, & 166 & -16 & 49 & 53 & -4 & 40 & \\
\hline
\end{tabular}


$L$ FO FC

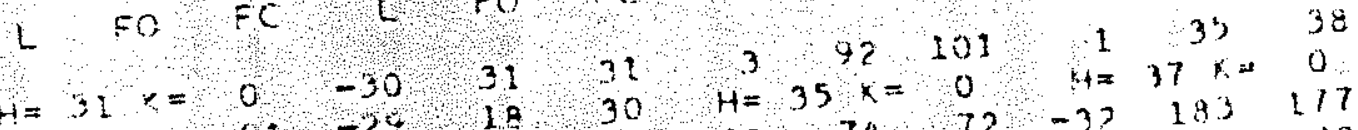

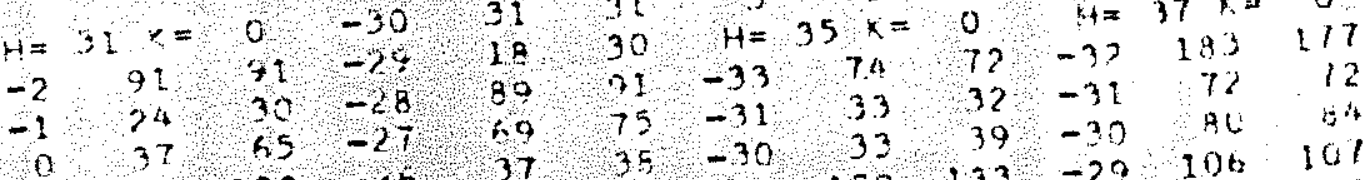

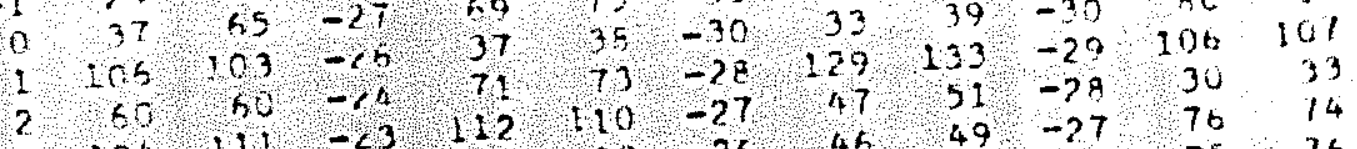

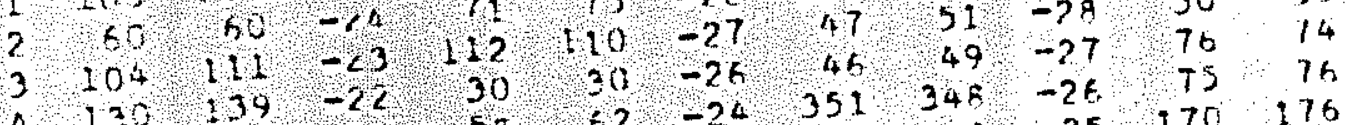

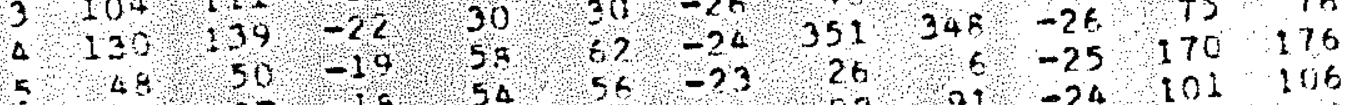

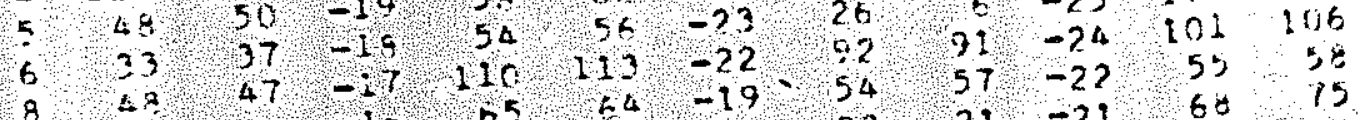

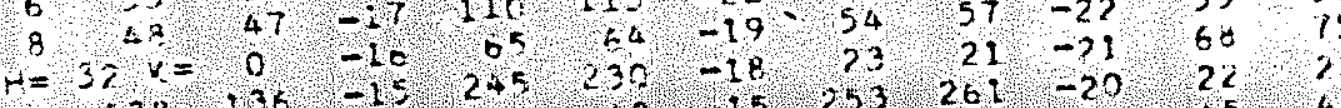

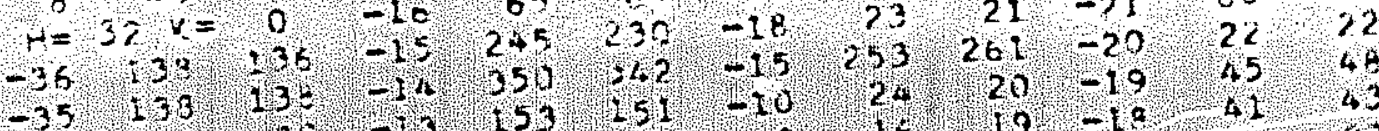

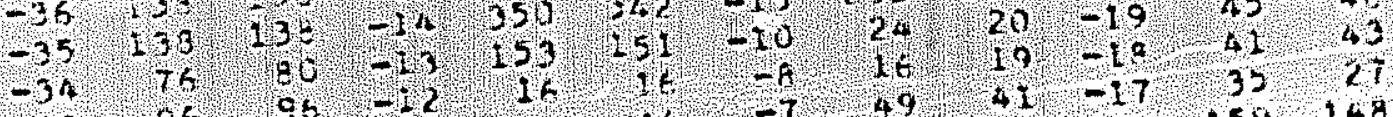

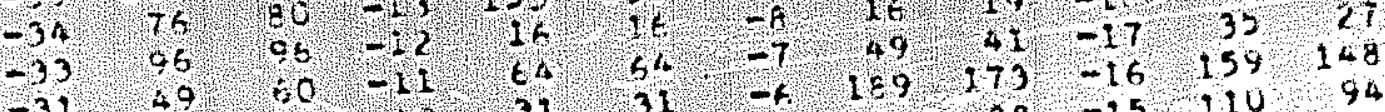

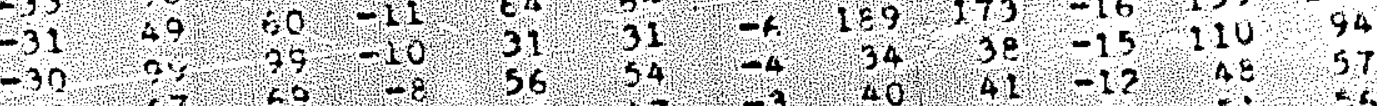

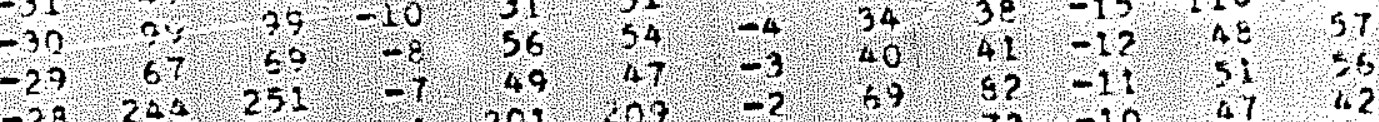

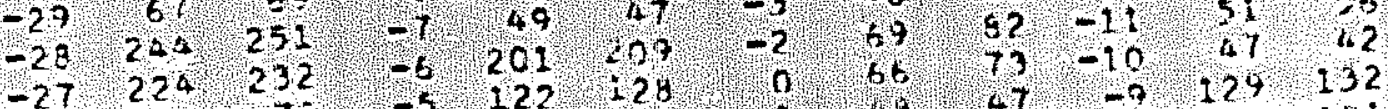

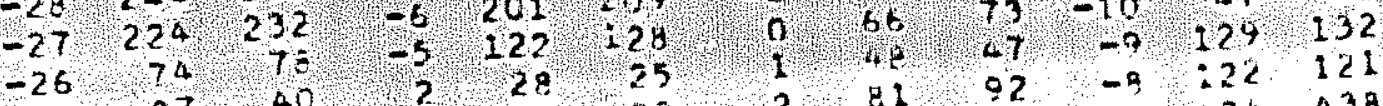

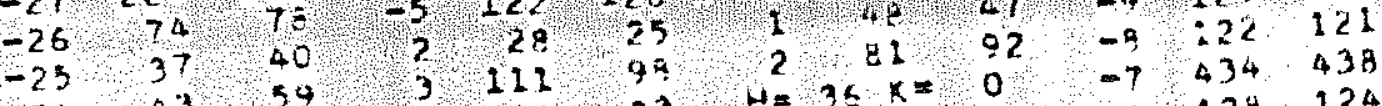

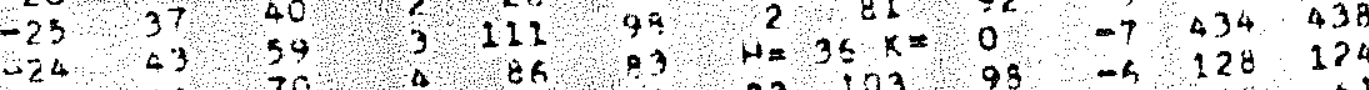

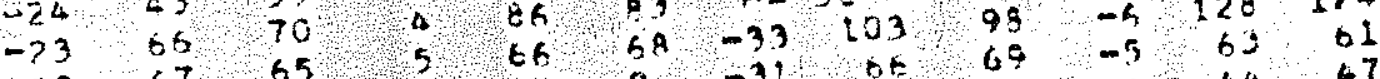

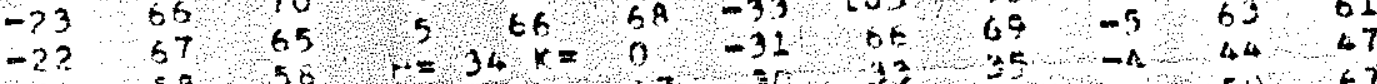

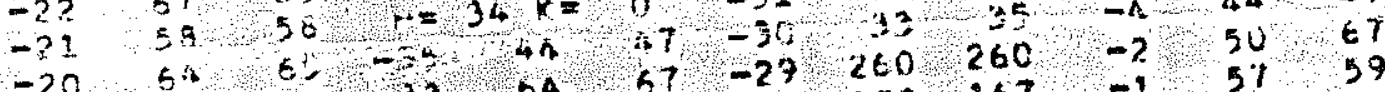

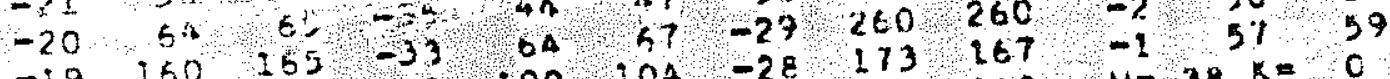

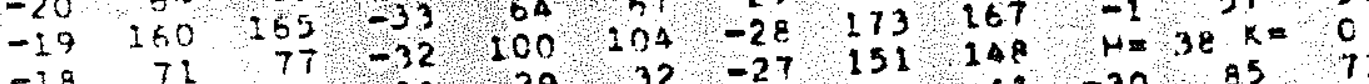

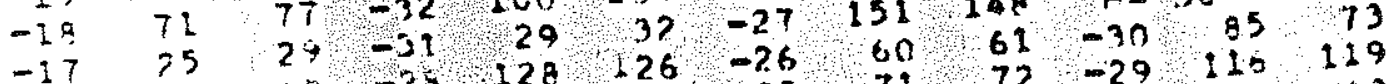

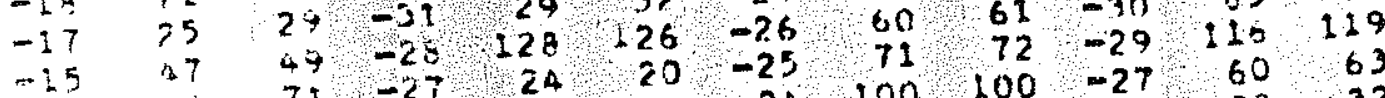

$\begin{array}{llllllll}-15 & 37 & 79 & -27 & 24 & 20 & -25 & \end{array}$

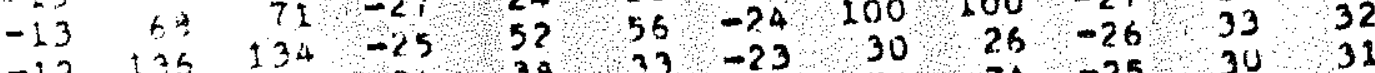

$\begin{array}{llllllllll}-12 & 136 & 134 & 132 & -24 & 38 & 33 & -23 & 3\end{array}$

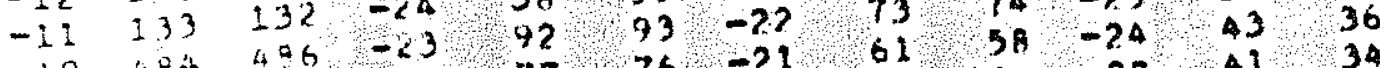

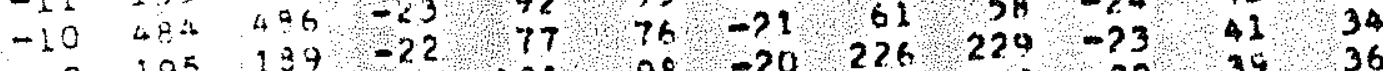

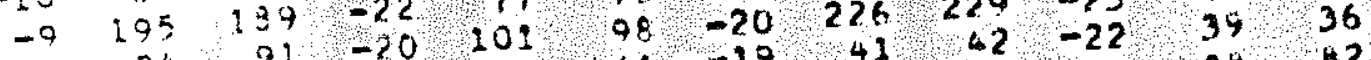

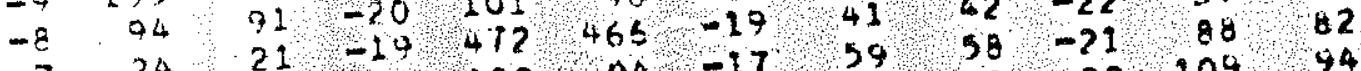

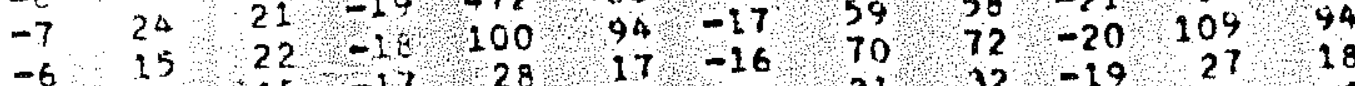

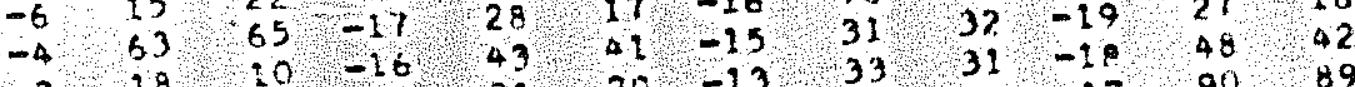

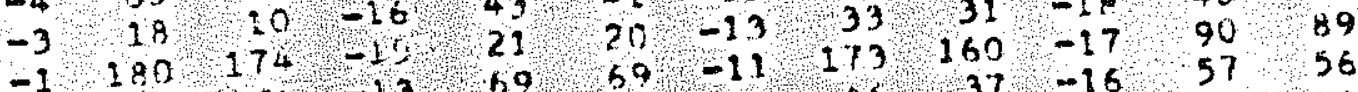

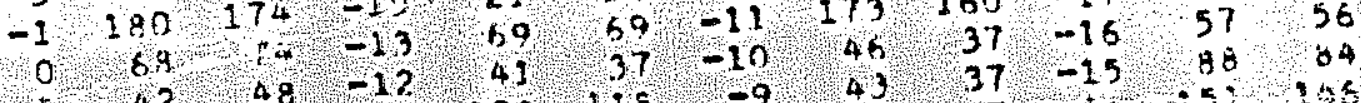

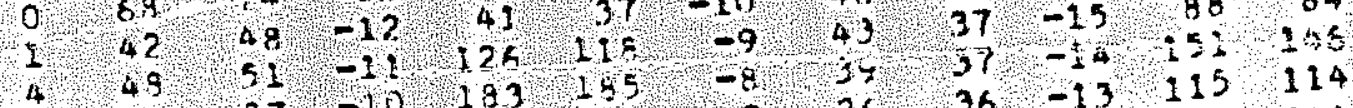

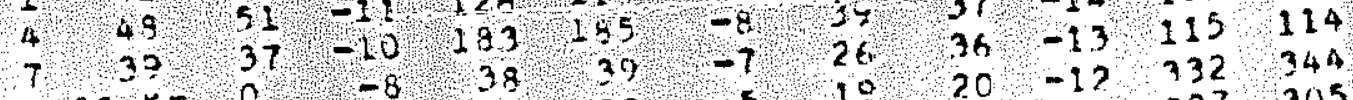

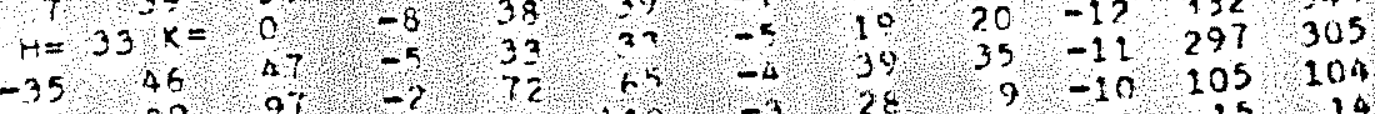

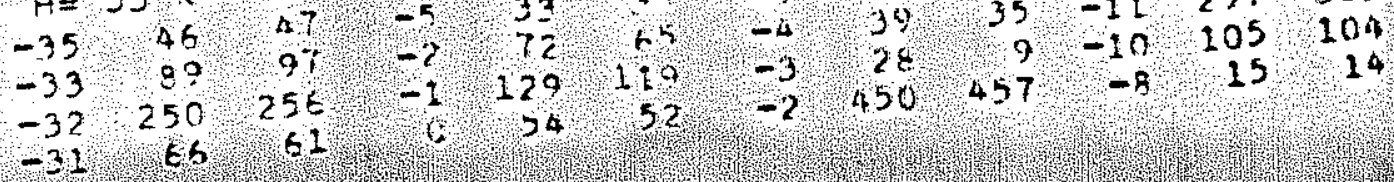




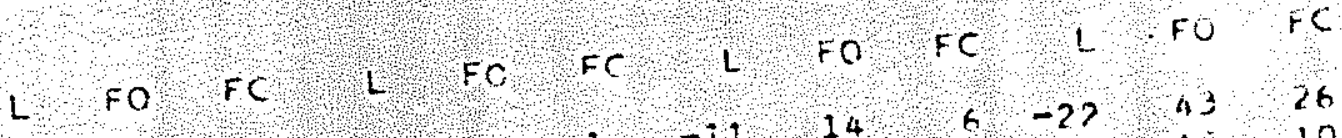

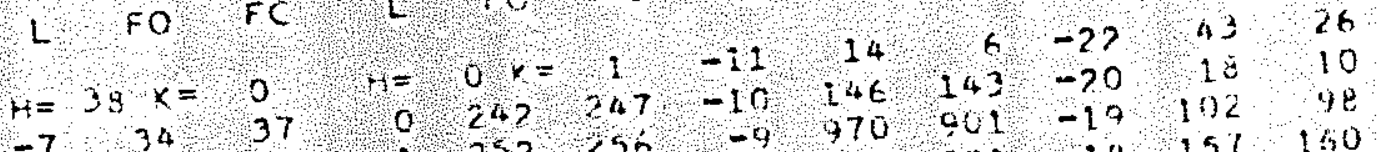

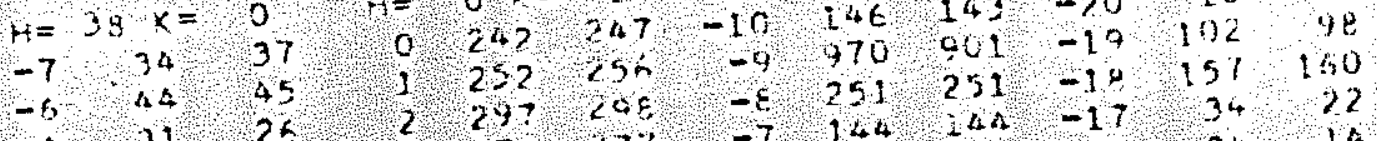

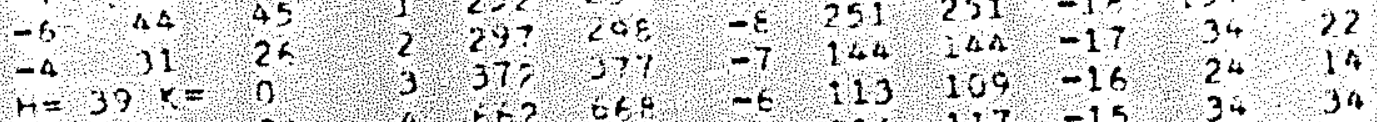

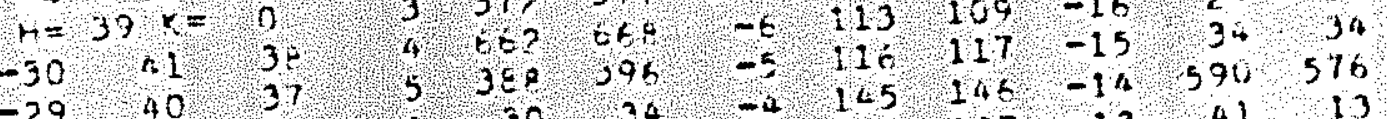

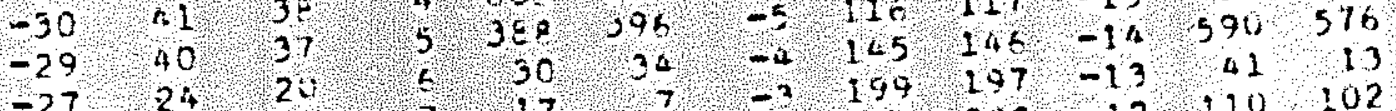

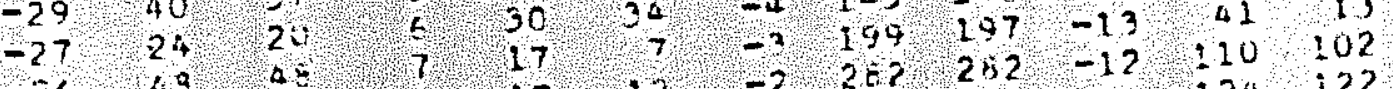

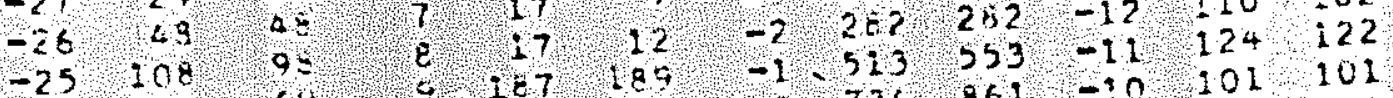

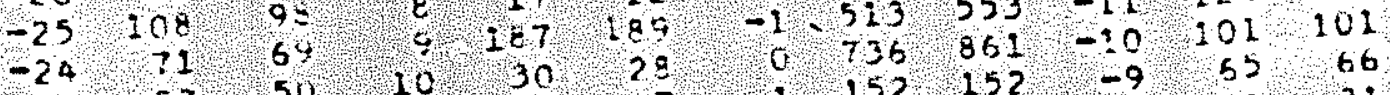

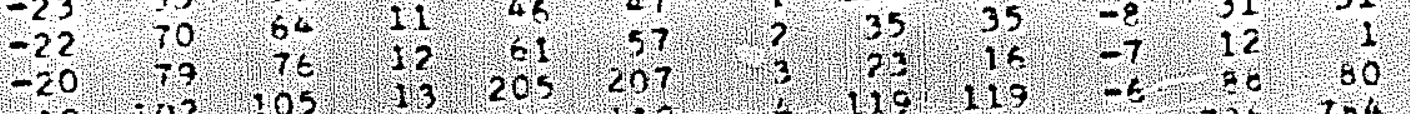

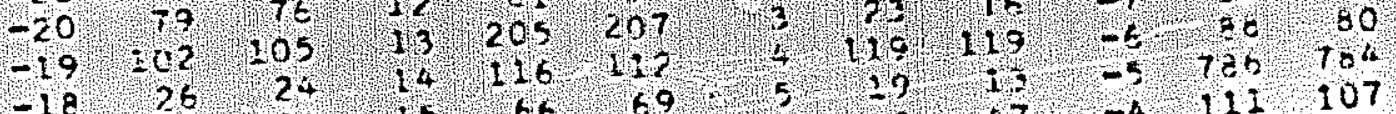

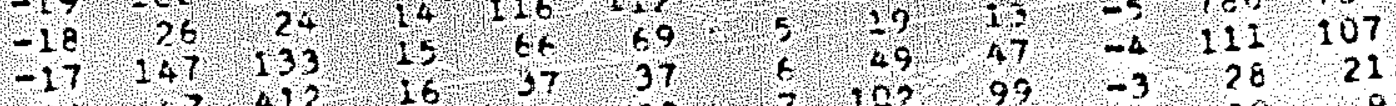

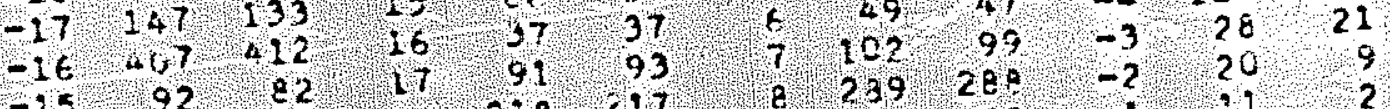

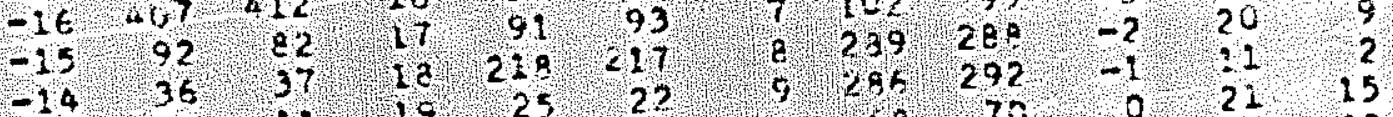

$\begin{array}{lllllllllllll}-10 & 26 & 1 & 19 & 25 & 23 & 40 & 69 & 70 & 0 & 21 & 15\end{array}$

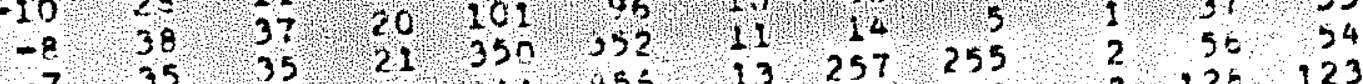

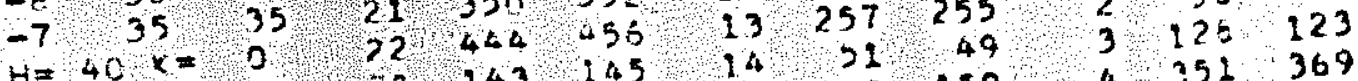

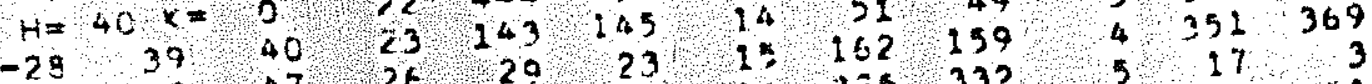

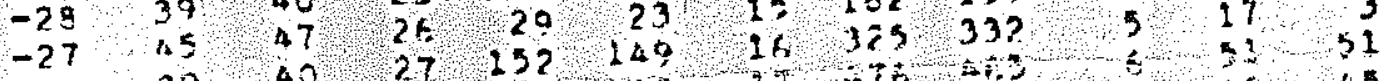

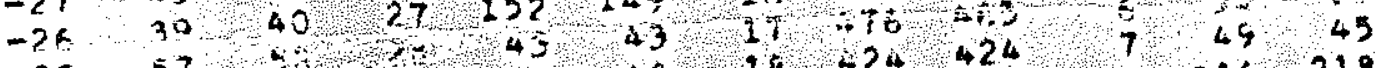

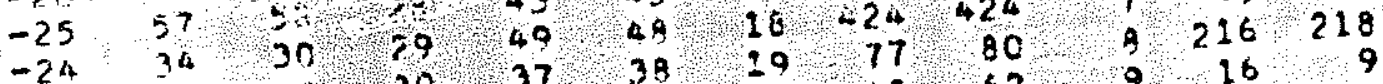

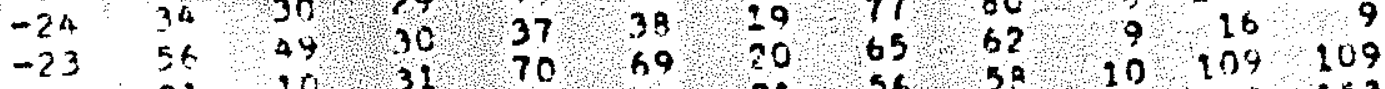

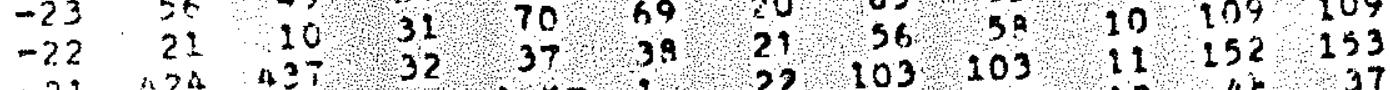

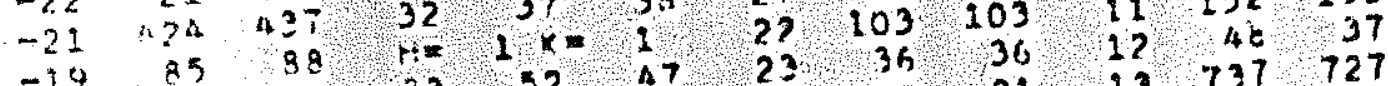

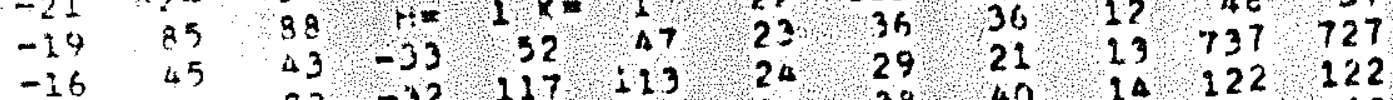

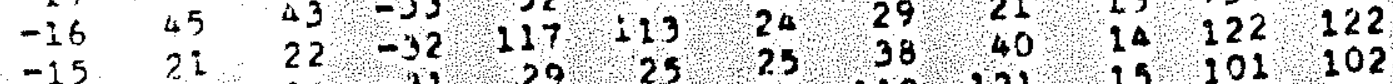

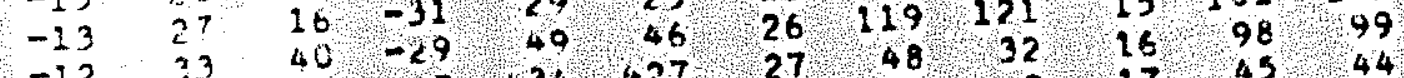

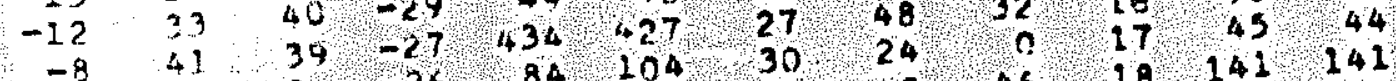

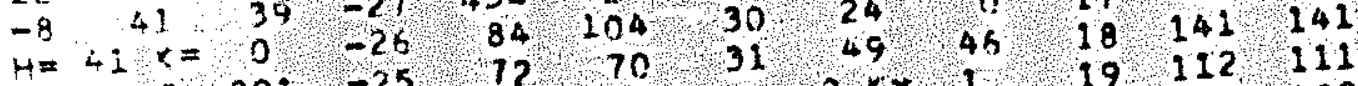

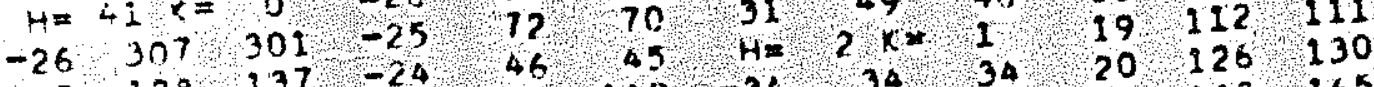

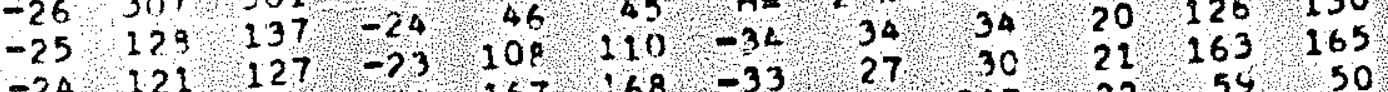

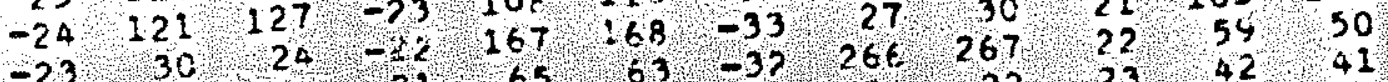

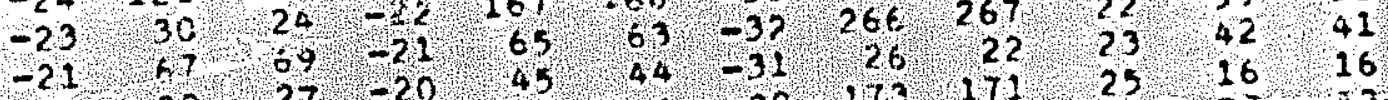

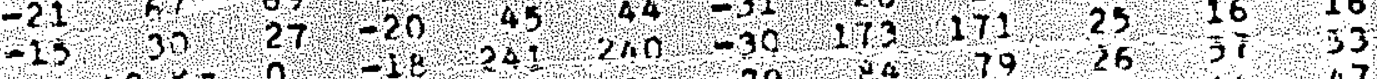

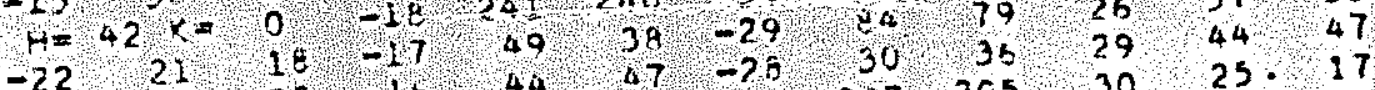

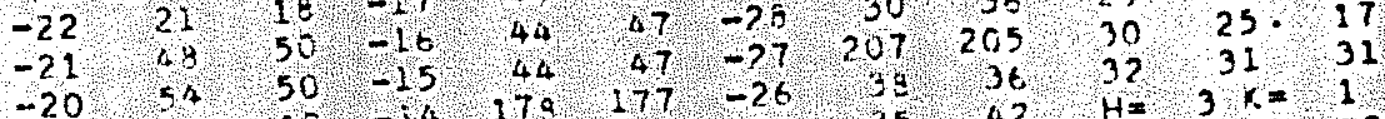

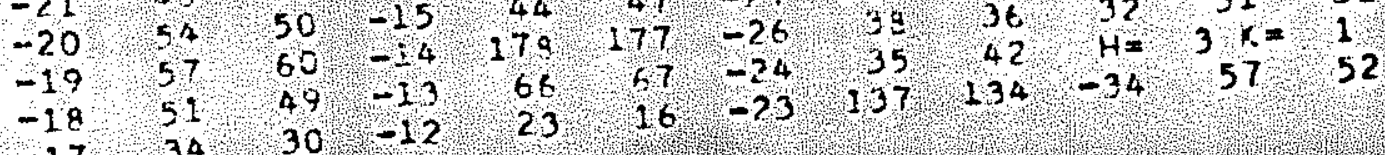




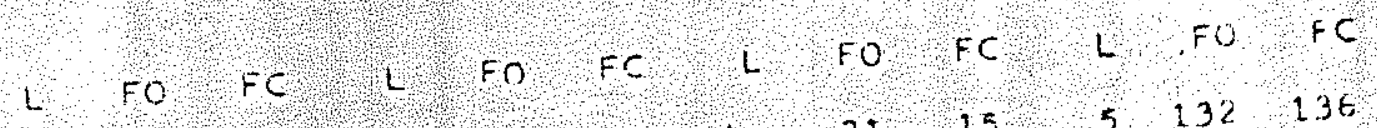

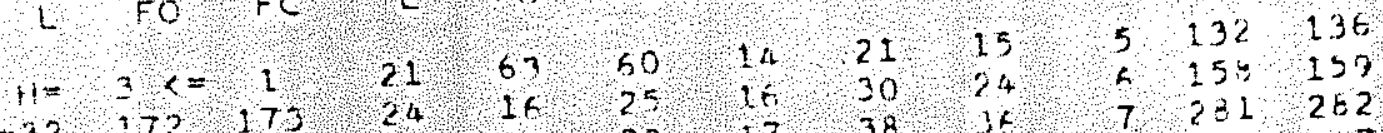

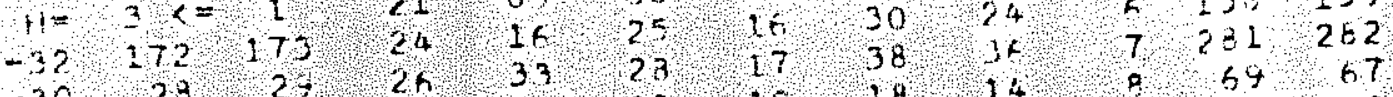

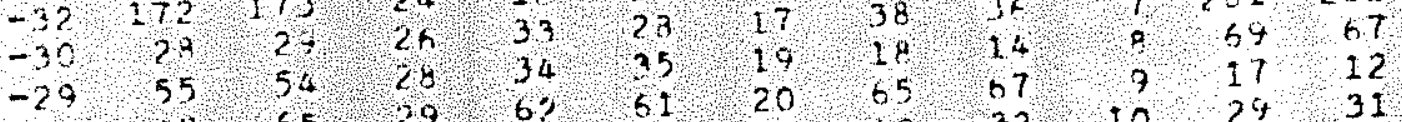

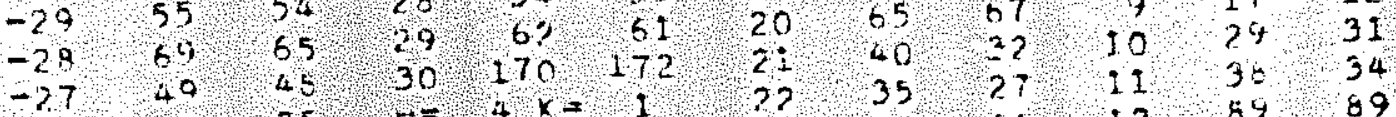

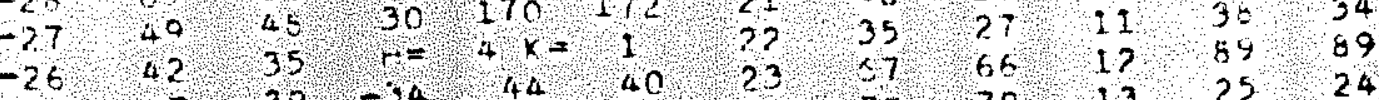

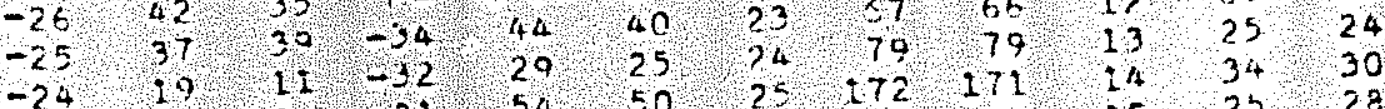

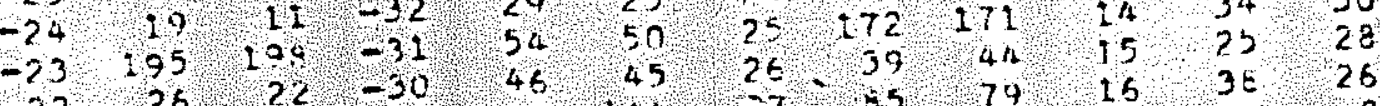

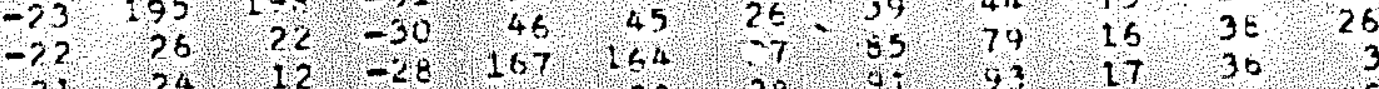

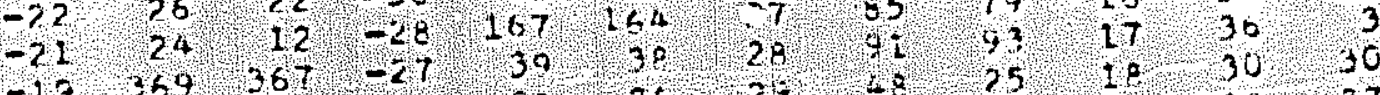

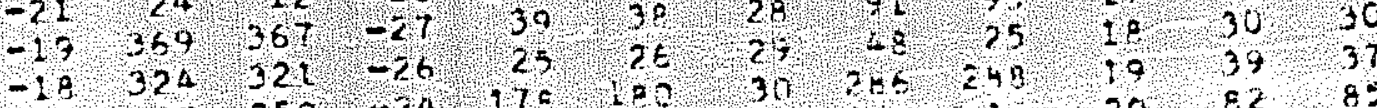

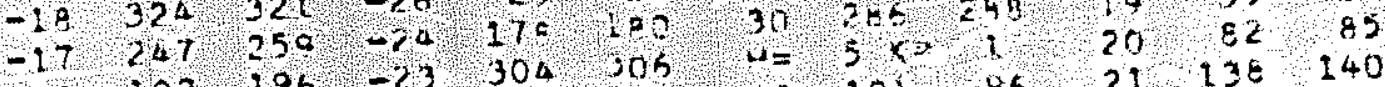

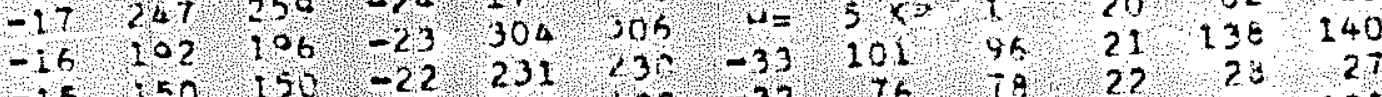

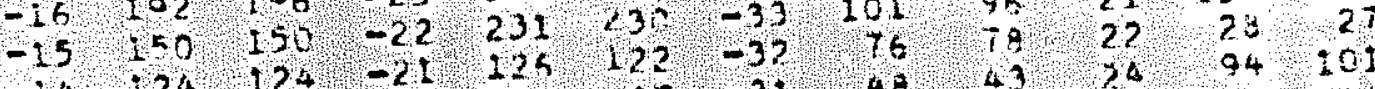

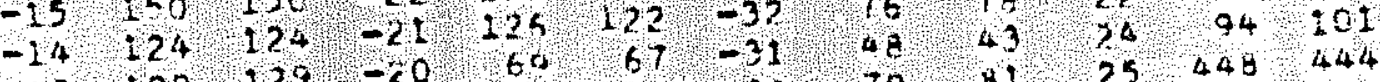

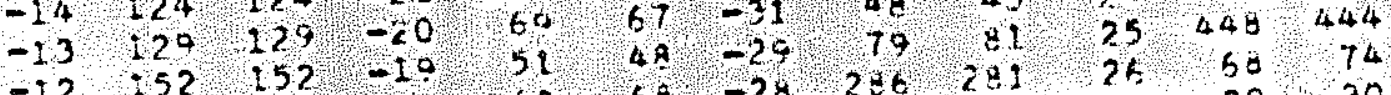

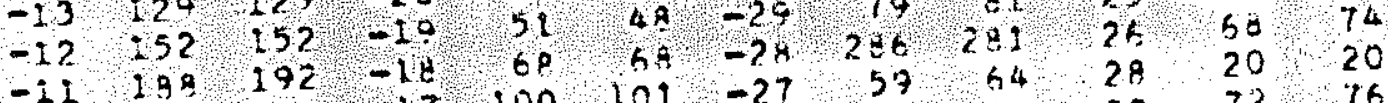

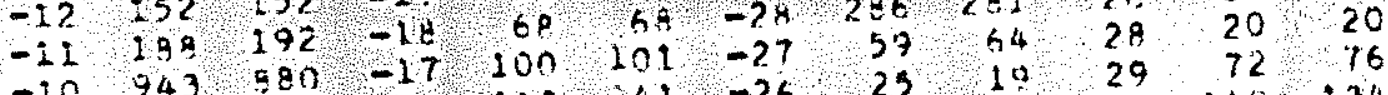

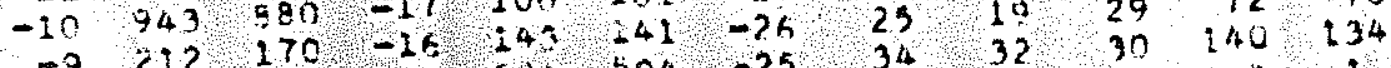

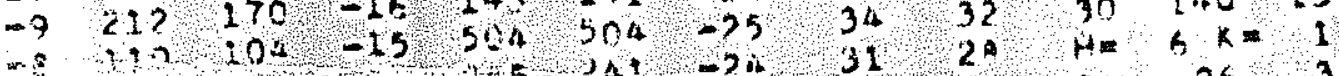

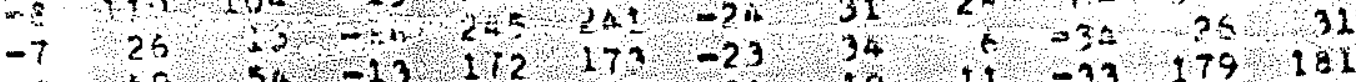

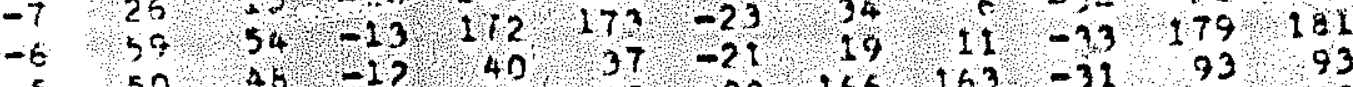

$\begin{array}{lllllllllll}-5 & 50 & 48 & -1 & 1 & 35 & 33 & -20 & 166 & 163 & -31 \\ -4 & 29 & 30 & -1 & 25 & -30 & 98\end{array} 85$

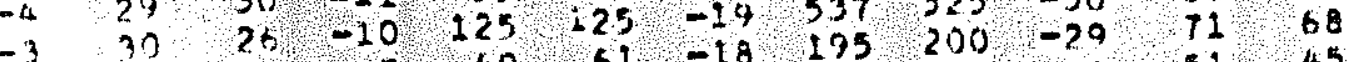

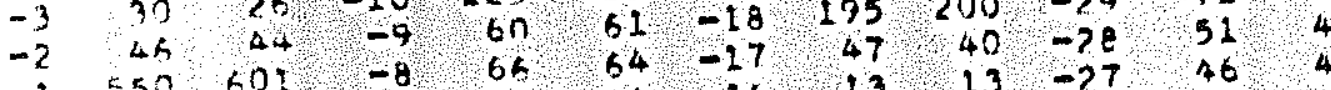

$\begin{array}{llllllllllll}-1 & 550 & 60 ! & -0 & 66 & 64 & -17 & 13 & 13 & -27 & 46 & 46\end{array}$

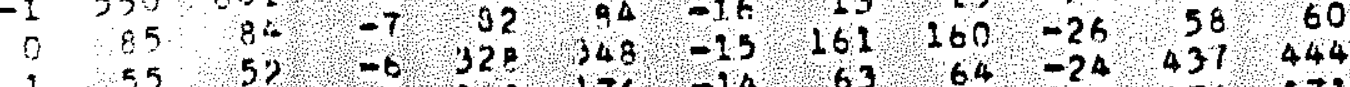

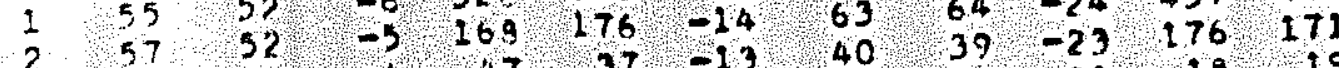

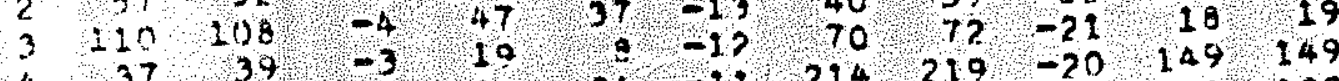

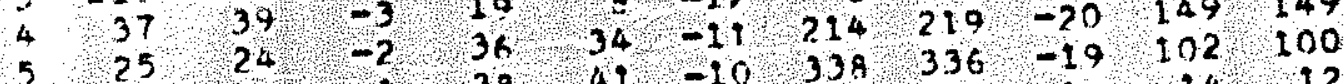

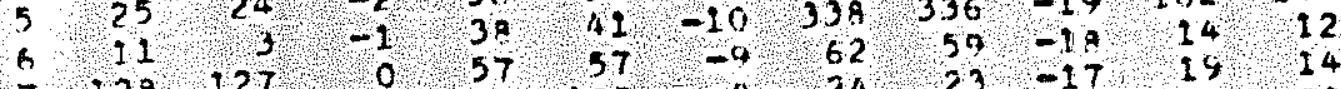

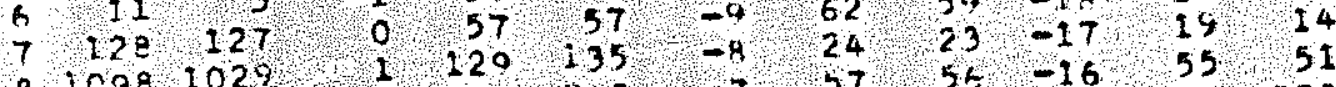

81038102 2 232 243 -7 ? $57,56-16,55451$

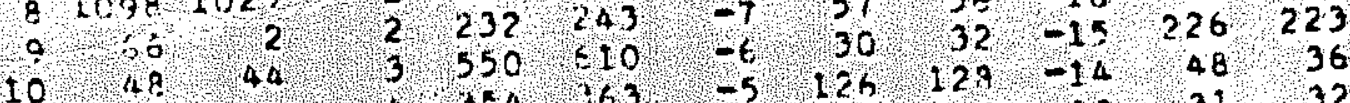

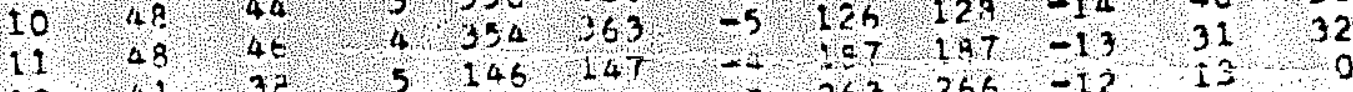

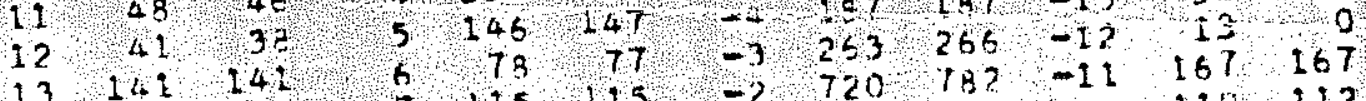

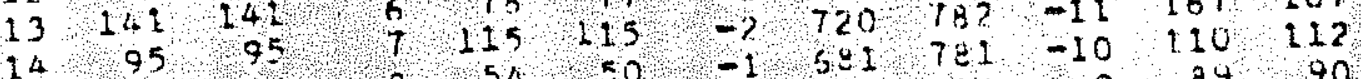

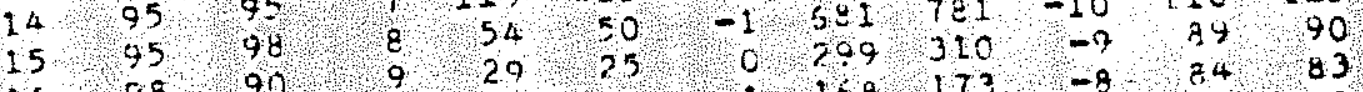

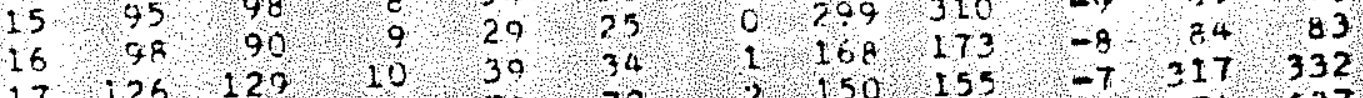

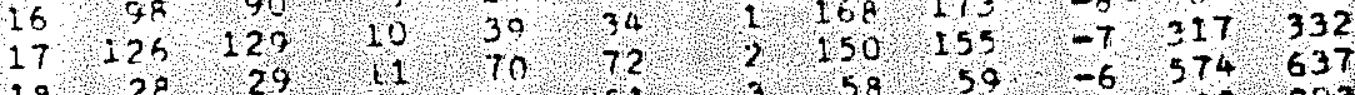

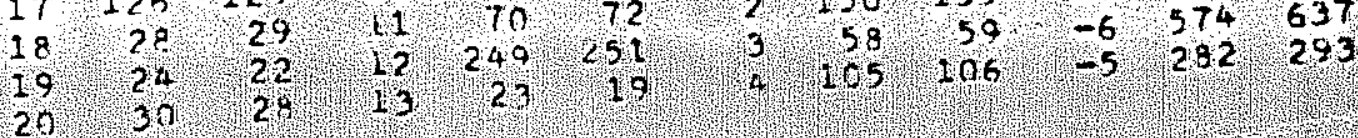




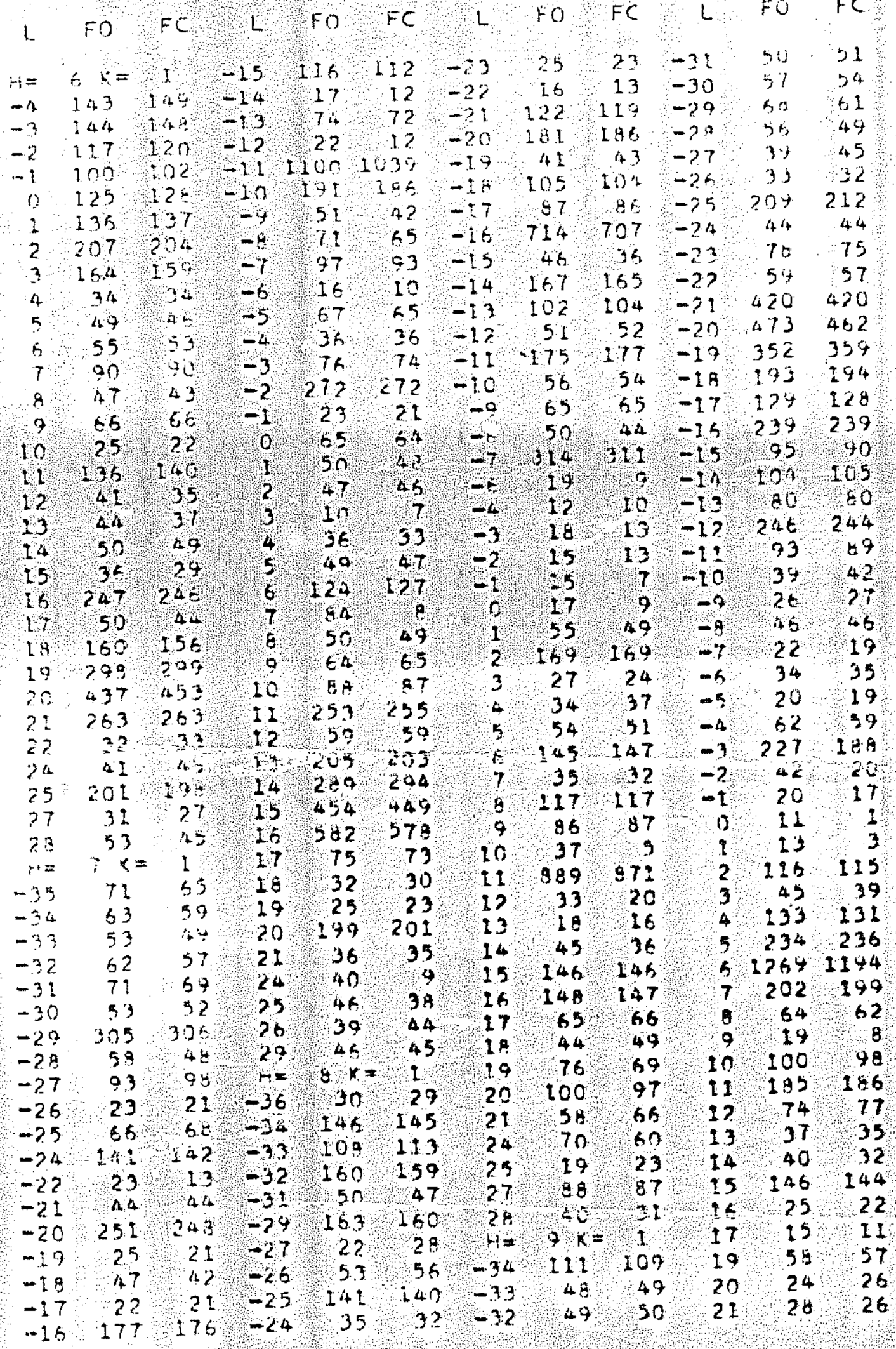




\begin{tabular}{|c|c|c|c|c|c|c|c|c|c|c|c|}
\hline L & $\mathrm{Fo}$ & $F C$ & t. & Fo & $1 c$ & L. & FO & $F C$ & I. & FU & FC \\
\hline$r=$ & $0 x=$ & 1 & 12 & 85 & a & $i_{4}$ & 18 & 15 & -1 & 47 & \\
\hline 22 & 146 & 43 & 13 & 47 & 42 & 5 & $i 01$ & 102 & 0 & 36 & \\
\hline 23 & 51 & 0,5 & 14 & 62 & 62 & 6 & 109 & 198 & 1 & 226 & \\
\hline 74 & 166 & 159 & 15 & 69 & 59 & 7 & 97 & 97 & $?$ & 69 & \\
\hline 26 & 47 & 47 & 17 & à & 18 & a & 64 & 61 & $?$ & 12 & \\
\hline 27 & 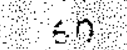 & 61 & 28 & 173 & 194 & 9 & 63 & 64 & 4 & 25 & \\
\hline 28 & 103 & 102 & $1 \%$ & 145 & 146 & 10 & 33 & 37 & 5 & 41 & 44 \\
\hline$+1=$ & $10 \quad t=$ & 1 & 20 & 32 & 41 & 12 & 45 & 47 & 6 & 17 & \\
\hline-34 & $85 \%$ & 50 & 21 & 41 & 10 & 13 & 134 & 135 & 7 & 20 & \\
\hline-33 & 89 & 45 & 32 & 55 & 55 & 14 & 147 & 129 & 8 & 44 & 4 \\
\hline 32 & 60 & 52 & 23 & 74 & 75 & 15 & 147 & 144 & $?$ & 113 & 13 \\
\hline 20 & 109 & 195 & $\angle 5$ & $1+7$ & 42 & 16 & 28 & 18 & 10 & 243 & 346 \\
\hline-19 & 28 & 36 & 27 & 72 & 5. & 19 & 140 & 100 & 11 & 7.76 & $9 \%$ \\
\hline$-3 \mathrm{e}$ & 1.3 & hs & $r=$ & $1+10=$ & 1. & 20 & 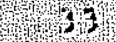 & $2 ?$ & 12 & 80 & \\
\hline 26 & 153 & 158 & $-3 s$ & 110 & $14 \%$ & 21 & +1 & 177 & 13 & 106 & 106 \\
\hline 25 & $4 \times 6$ & 483 & -24 & 112 & 172 & 22 & 137 & 141 & 14 & 172 & 17 \\
\hline-24 & 250 & 356 & -0 & 49 & 2,2 & 23 & 5,79 & 301 & 15 & 80 & \\
\hline-23 & 414 & 111 & -37 & 26 & 27 & 24 & tor & 111 & 16 & 172 & 27 \\
\hline$-2 ?$ & 51 & 53 & -31 & 17 & 23 & $2 y$ & $<4$ & 23 & 17 & 223 & 21 \\
\hline-21 & 11.6 & 50 & -30 & 442 & 40 & 27 & 29 & 29 & 18 & 248 & 2 \\
\hline-20 & $13 ?$ & 35 & -29 & 04 & 100 & 110 & $12 \%$ & 1 & $1 ?$ & 269 & 26 \\
\hline-13 & 72 & 17 & -24 & 79 & 75 & $-3 \%$ & $\begin{array}{r}30 \\
\end{array}$ & 30 & 21 & 28 & 28 \\
\hline - is & 29 & 19 & -27 & 58 & 53 & -35 & 244 & 244 & 23 & $17 \%$ & 182 \\
\hline 17 & 221 & 127 & -26 & 31 & 25 & -33 & 191 & 189 & 26 & 31 & 1 \\
\hline-15 & $=3$ & 15 & -25 & 132 & 13 & -32 & 120 & 114 & $4=$ & $13 \mathrm{k}=$ & \\
\hline-15 & 71 & 76 & -24 & 270 & 168 & -31 & 36 & 34 & -37 & 83 & \\
\hline-14 & 65 & 3 & $-7,3$ & 59 & 56 & -30 & 164 & $1 \in 7$ & -36 & 50 & 4 \\
\hline-13 & 36 & 34 & -21 & $19 ?$ & 291 & -29 & 663 & 62 & -35 & 130 & 13 \\
\hline 12 & 15 & 8 & -20 & 35 & 32 & -25 & 54 & 52 & -34 & 34 & \\
\hline-11 & 57 & 56 & -19 & 40 & 45 & -27 & 27 & 18 & -31 & 105 & 106 \\
\hline-10 & 43 & 41 & -1 H & 32 & 26 & -26 & 181 & 174 & -30 & 56 & 44 \\
\hline-5 & 70 & 69 & -17 & 33 & 16 & -24 & 46 & 46 & -27 & 26 & 10 \\
\hline$-\hat{3}$ & 131 & 137 & -16 & 50 & 10 & -22 & 51 & 50 & -28 & 35 & \\
\hline-7 & 67 & 93 & -15 & 41 & 15 & -21 & 19 & 0 & -27 & 53 & \\
\hline$-t$ & 9.2 & 78 & -24 & $5 \%$ & 50 & -20 & 45 & 37 & -25 & 52 & \\
\hline-5 & $1: 0$ & 109 & -13 & 36 & 37 & -18 & $t 3$ & 44 & -24 & 34 & 6 \\
\hline-4 & 237 & 136 & -12 & 80 & 92 & -17 & 103 & 94 & -23 & 83 & \\
\hline-3 & 174 & 123 & -11 & 34 & 56 & $-1 h$ & 42 & $? 9$ & -22 & 83 & \\
\hline-2 & 132 & 136 & -10 & 49 & 49 & -15 & 55 & 53 & -21 & 40 & \\
\hline-1 & 263 & 267 & -9 & $9 ?$ & 14 & $=14$ & 74 & 72 & -20 & 93 & 86 \\
\hline 0 & 341 & 343 & -8 & 23 & 13 & -13 & 19.1 & 190 & -19 & 90 & \\
\hline 1 & 870 & 971 & -7 & 135 & 136 & -12 & 41 & 44 & $-1 R$ & 180 & 177 \\
\hline 2 & 617 & 635 & -6 & 199 & 201 & -11 & 16 & 14 & -17 & 65 & 6 \\
\hline 3 & 101 & 195 & -5 & 240 & 230 & -20 & 45 & 45 & $-1 \mathrm{~A}$ & 36 & \\
\hline 4 & 55 & 54 & -4 & 662 & 661 & -9 & 149 & 091 & -13 & 177 & 113 \\
\hline 5 & 104 & 101 & -3 & 1085 & $114 ?$ & -8 & 1174 & 1124 & -14 & 181 & 18 \\
\hline 6 & 129 & 129 & $=-2$ & 327 & 326 & -7 & 160 & 160 & -13 & 1188 & 114 \\
\hline 7 & 12 & 11 & -1 & 108 & 108 & -6 & 30 & 25 & -12 & 275 & 27 \\
\hline 3 & 19 & 12 & 0 & 99 & 98 & -5 & 02 & 79 & -11 & 185 & 18 \\
\hline 9 & 19 & 44 & 1 & 34 & 22 & -4 & 50 & 48 & -10 & 37 & \\
\hline & 63 & 63 & & 50 & 59 & $x^{4}$ & 43 & 32 & -9 & 37 & 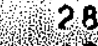 \\
\hline & 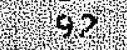 & 84 & & 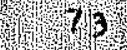 & 18 & $m$ & 4 & 31 & 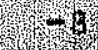 & 61 & 62 \\
\hline
\end{tabular}




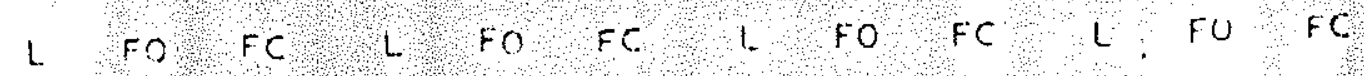

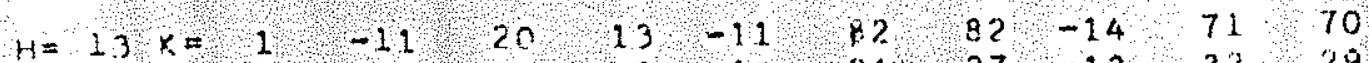

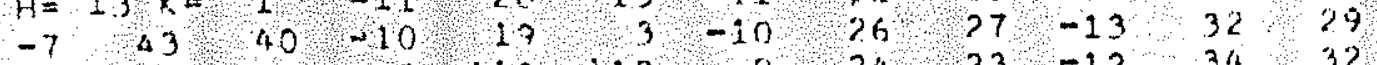

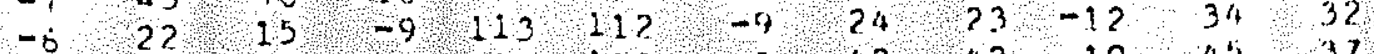

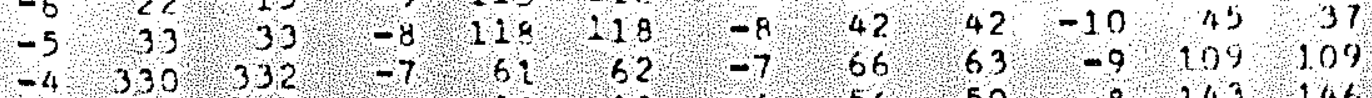

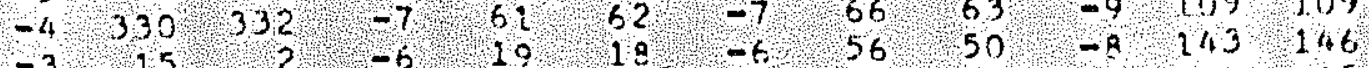

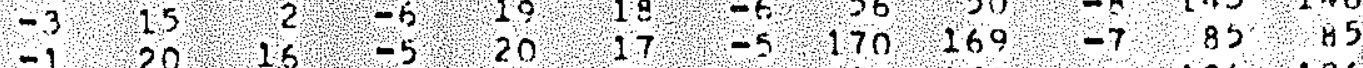

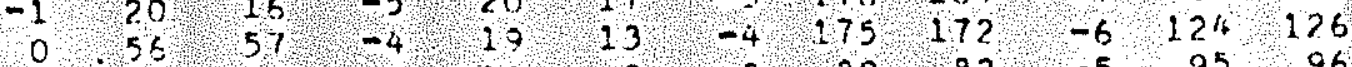

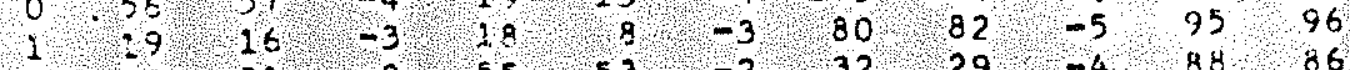

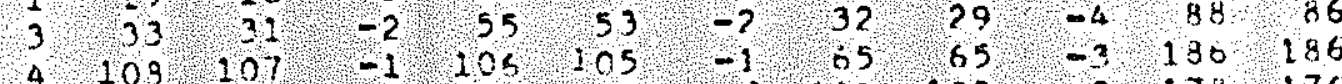

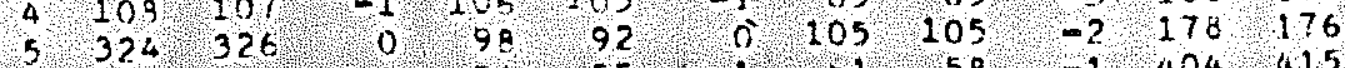

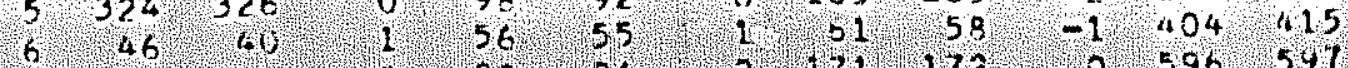

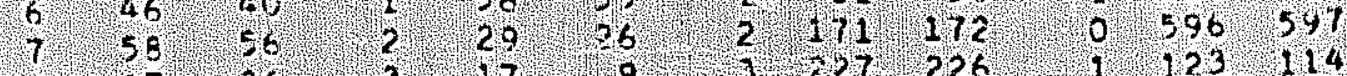

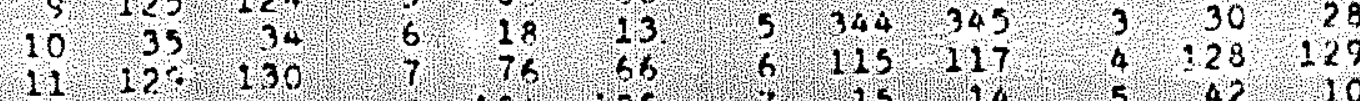

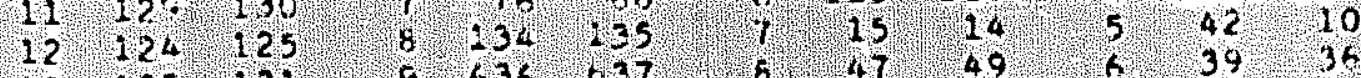

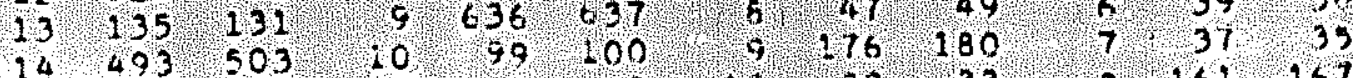

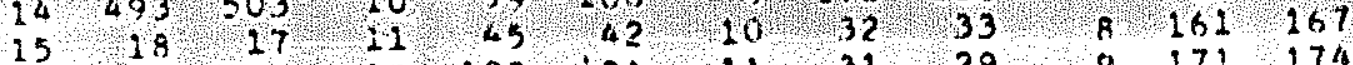

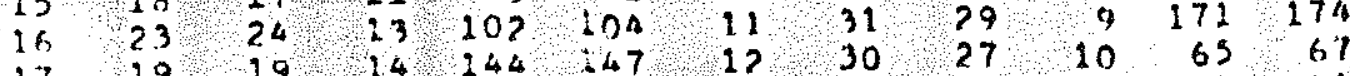

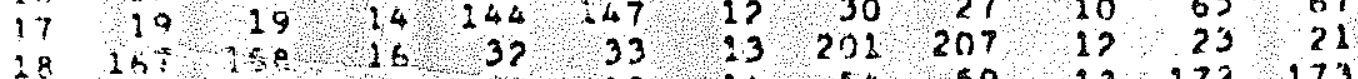

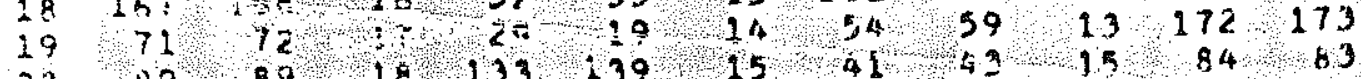

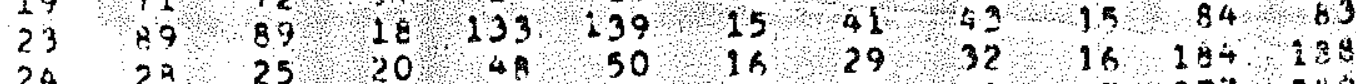

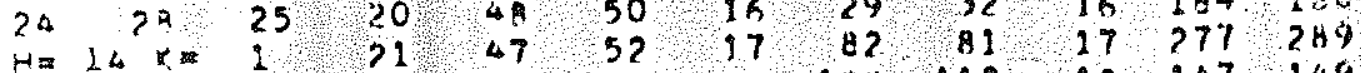

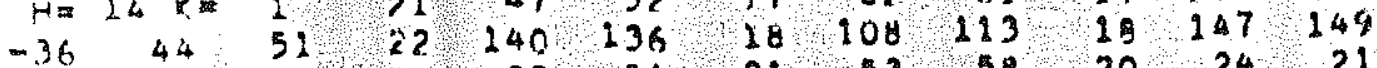

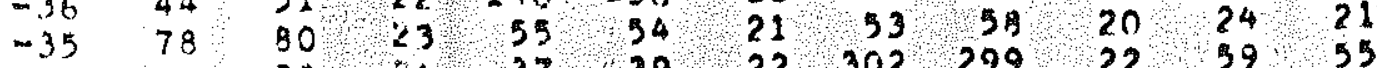

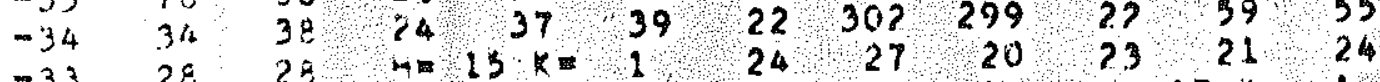

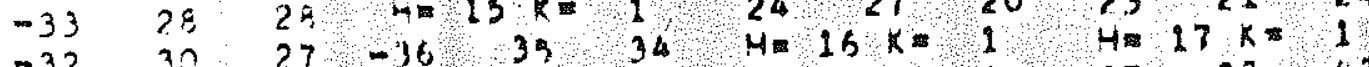

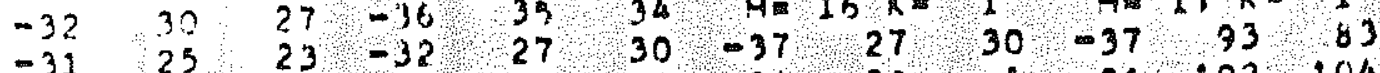

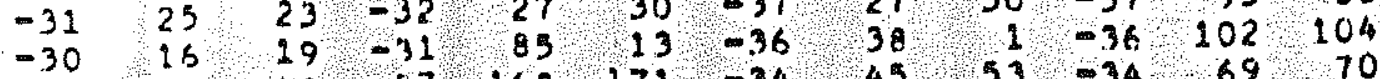

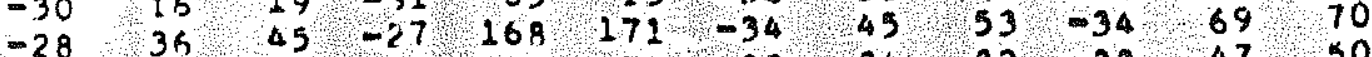

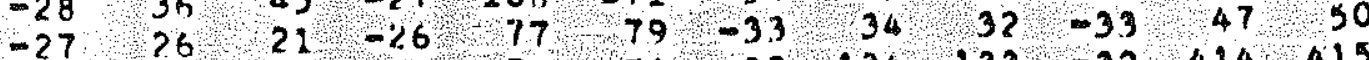

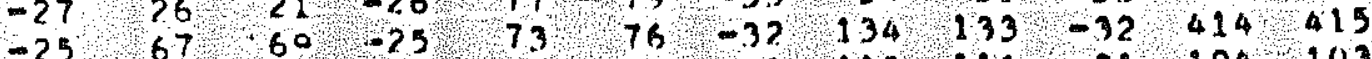

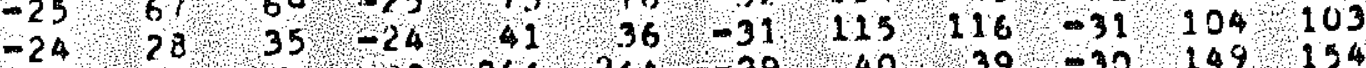

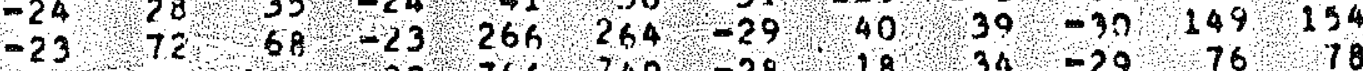

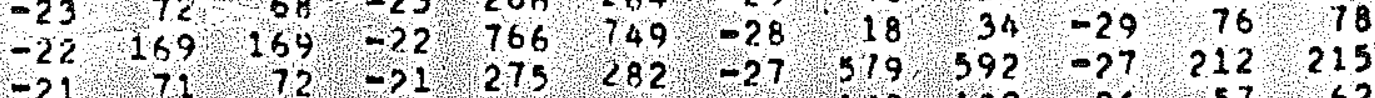

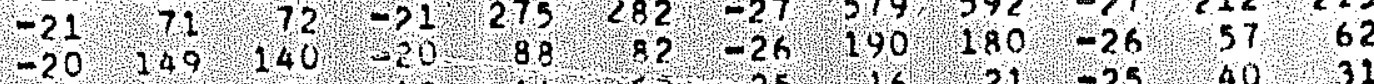

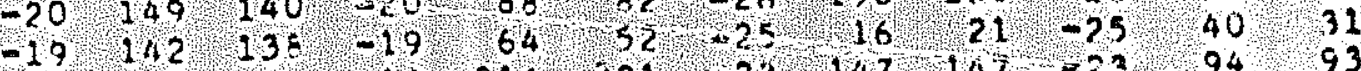

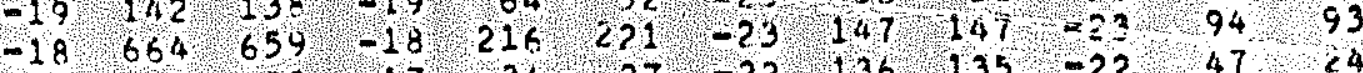

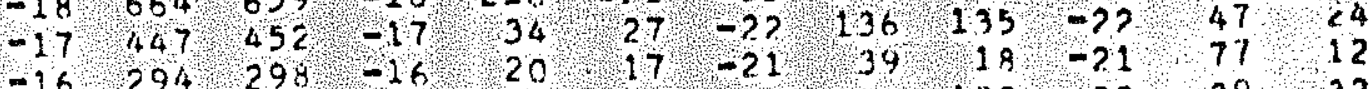

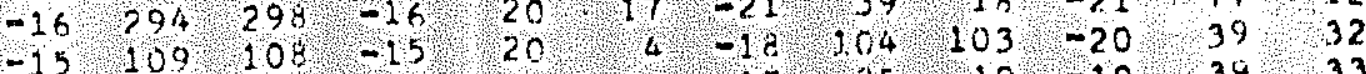

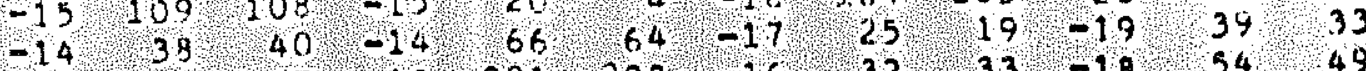

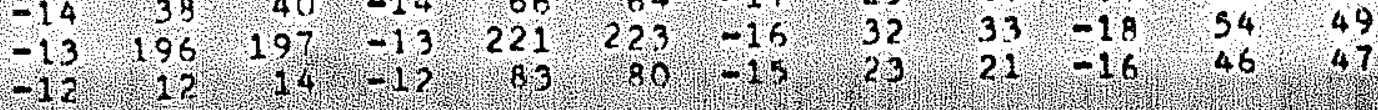




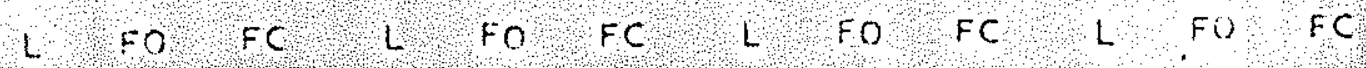

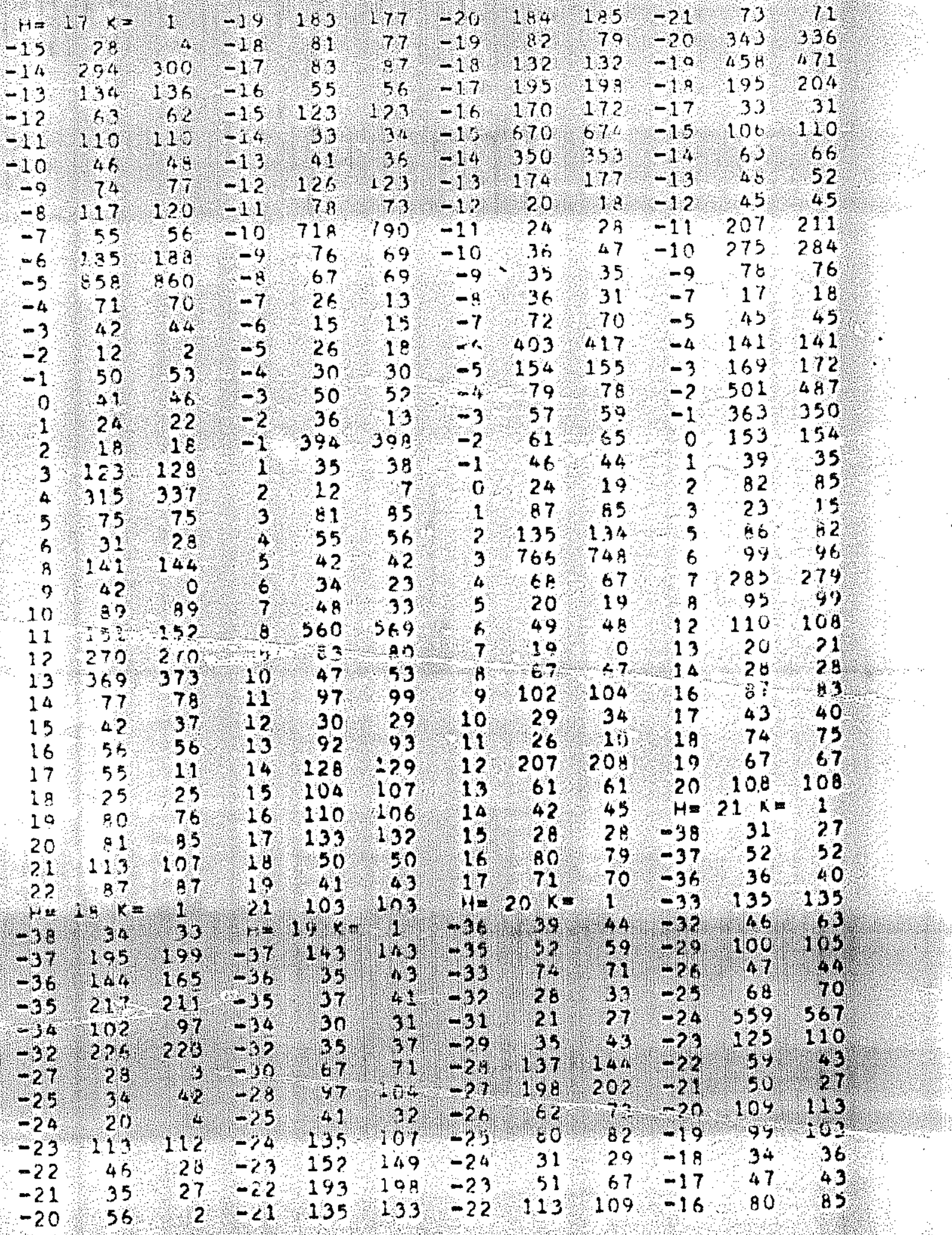


L

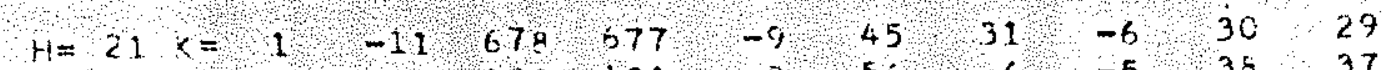

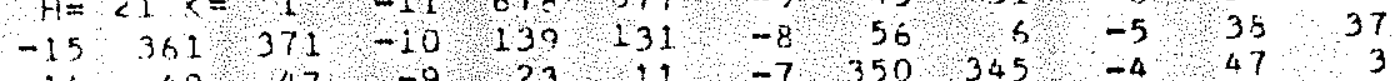

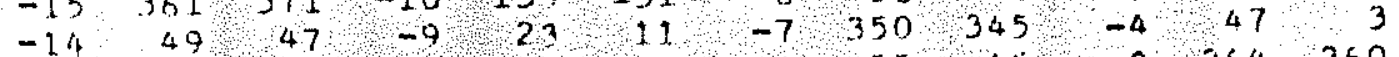

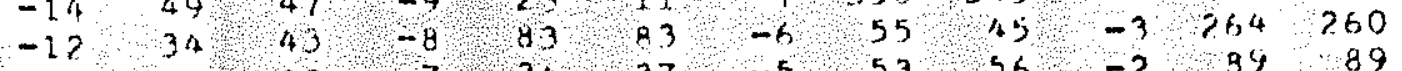

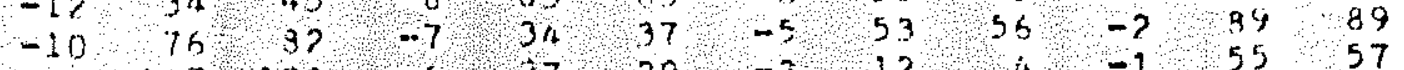

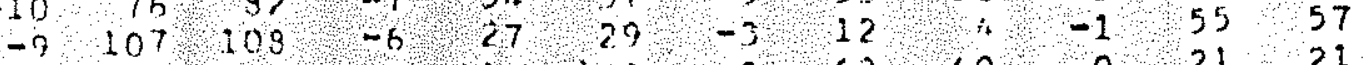

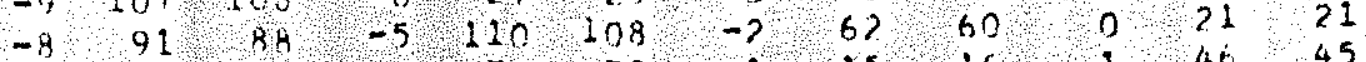
$-7 \times 337335$

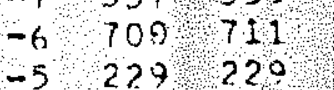

-5
$-4,89$
-3

$-4,76,73$

$-3 \quad 143$ 141

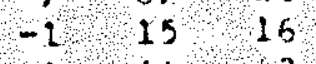

$-2 \quad 201289$

$-3,123<131$

$\begin{array}{lll}-1 & 23 & 15 \\ 0 & 33\end{array}$

$-2,65$
-1
-1

$-1,59 ? \begin{array}{ll}56 \\ 0\end{array}$

2 470 ? 70

127,126

240.235

(3) 111 1 111

$1 \% 64$ क3

? 2109205

4,40 ४ 41

5 48 49

$6,144,147$

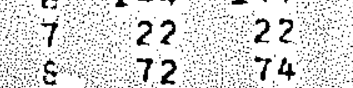

149,145

4. 7 4 V T?

42,45

1. 270

67

1

43043

11

95. 107

12

46

469

1 सम 45

$22881 \quad 90$

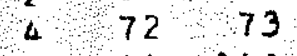

$5144 \cdot 146$

$6 \longdiv { 5 3 7 } 5 4 4$

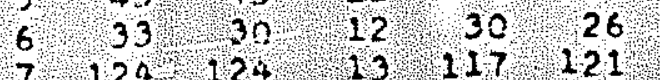

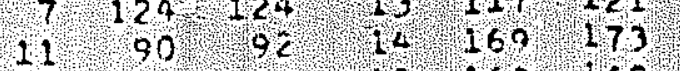

15.

2

4,12

(30. 13

$70 \% 61$

$4 0 \longdiv { 3 5 }$

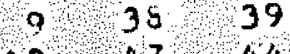

$10,47, \quad 44$

12.45 .43

13
15
154

15 . 160160

16

14

75 .

$5 5 \longdiv { 5 2 }$

$\begin{array}{lllll}16 & 66 & 63 & 4 \pi & 2 \\ 17 & 57 & 60 & -37 & 30\end{array}$

16. 256,261

$4=247$.

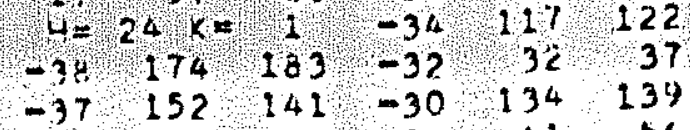

$16 \quad 149 \quad 251$ $\mathrm{H}=23 \mathrm{rr}+1,-$

141 \%

$-32$

$134 \quad 134$

$22 \div 22-20$

4136

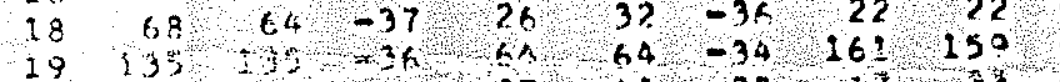

$\begin{array}{ccc}H= & 22 & K=1 \\ -39 & 76 & 80\end{array}$

$\begin{array}{lll}-38 & 76 & 80 \\ -36 & 104 & 111\end{array}$

$\begin{array}{lll}-35 & 64 & 57\end{array}$

$\begin{array}{llc}-34 & 105 & 105 \\ -31 & 79\end{array}$

$\begin{array}{lll}-31 & 78 & 79 \\ -20 & 418 & 425\end{array}$

प5 37

$41,-33$

17

$3+$

-28
-25

$60 \quad 62$

$253,-30$

74
96
23

151 .

$152,-25$

126

$133,-28$

59. -27

145 ? 151 ? $35-25$

34

107

$-25$

1572136

4 द1 481

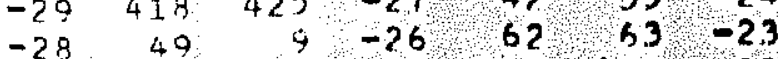

$-27 \quad 91 \quad 101 \%-25 \quad 19 n$ 189 -27

21

13

$-24$

$203 \quad 208$

$\begin{array}{lllllll}-26 & 36 & 34 & -14 & 105 & 112 & -2\end{array}$

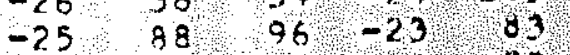

$-24 \quad 133<136 \quad-22$

$-23 \quad 31$

$\begin{array}{ll}-22 & 31 \\ -2 & 74\end{array}$

35

$36,-20$

8

181

$-22$

9480

-21 \% 108 \% 107

$16-20 \quad 37 ? 45$

$346 \quad 351 \quad-17$ ? 264 259

293 297 -16 171 169

67 . $68,-1$

238

$-15$

$140 \quad 38$

$-21,27,15,-19$,

$98 \times 101,-17$, 121

12

103

$-14$

63
56
5

$2 4 \longdiv { 2 5 }$

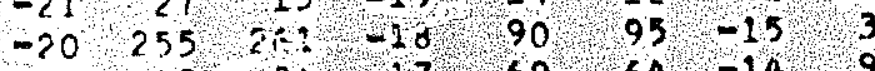

$117-11$

$-11$

$30 \lcm{30}$

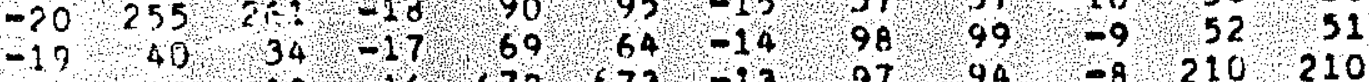

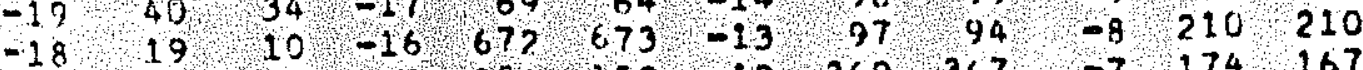

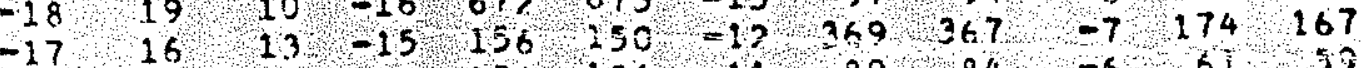

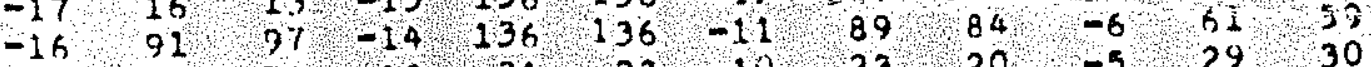

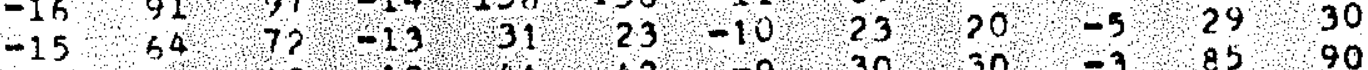

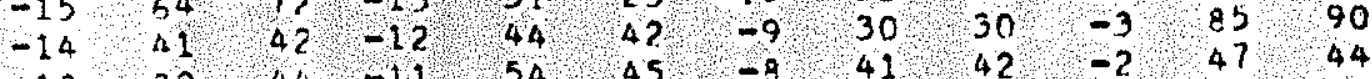

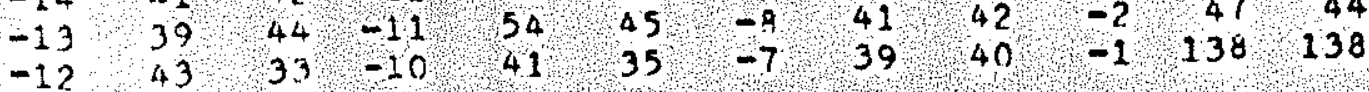




\begin{tabular}{|c|c|c|c|c|c|c|c|c|}
\hline L & $\mathrm{FO}$ & $F C$ & $L$ & ro & $\mathrm{FC}$ & $L$ & $\mathrm{FO}$ & $F C$ \\
\hline $\begin{array}{l}-32 \\
-31\end{array}$ & 57 & 60 & -72 & 165 & 163 & -1 & 36 & 20 \\
\hline $\begin{array}{l}-31 \\
-30\end{array}$ & 51 & 55 & -21 & 108 & 105 & 0 & 141 & 130 \\
\hline $\begin{array}{r}-30 \\
-79\end{array}$ & 84 & 92 & $->0$ & 41 & 15 & 1 & 441 & 127 \\
\hline $\begin{array}{l}-79 \\
-83\end{array}$ & 35 & 30 & -18 & 77 & 83 & 2 & 29 & 89 \\
\hline-20 & 62 & 51 & -17 & $4+5$ & 54 & 3 & 51 & 48 \\
\hline 26 & 36 & 29 & -15 & 117 & 110 & 4 & 57 & 63 \\
\hline 26 & 276 & 279 & -12 & 74 & 159 & 5 & 31 & 33 \\
\hline 24 & 447 & 27 & -13 & 636 & 638 & 6 & 68 & 64 \\
\hline 22 & 43 & 48 & -12 & 176 & $3 n$ & 9 & .34 & 30 \\
\hline 22 & 67 & 69 & -11 & 80 & 82 & 9 & 347 & 344 \\
\hline $\begin{array}{l}-21 \\
-12\end{array}$ & 54 & 57 & -10 & 19 & 22 & 10 & 36 & 40 \\
\hline $\begin{array}{l}-12 \\
-17\end{array}$ & 13 & 21 & - & 49 & 46 & $H=$ & $30 \mathrm{k}=$ & 1 \\
\hline-17 & $21 \%$ & 217 & -6 & 31 & 33 & -36 & 72 & 14 \\
\hline-16 & 69 & 61 & -5 & 34 & 33 & -35 & 31 & 33 \\
\hline-15 & 65 & 67 & (19-4 & 36 & 32 & $-8 ?$ & 18 & C6 \\
\hline-14 & 104 & 103 & -3 & 27 & 21 & -31 & +3 & 27 \\
\hline 13 & 39 & 40 & -1 & 27 & 7 & -28 & 41 & 5 \\
\hline-12 & 80 & 94 & 5 & 93 & 88 & -27 & 111 & 116 \\
\hline-11 & 109 & 206 & 6 & 64 & 59 & -26 & 52 & 71 \\
\hline-10 & 29 & 30 & 7 & 69 & 67 & 225 & 107 & 10 \\
\hline-9 & 147 & 150 & 9 & 86 & 65 & -24 & 30 & 87 \\
\hline-9 & 677 & 703 & 9 & 124 & 124 & -23 & 245 & 253 \\
\hline-7 & 99 & 94 & 12 & 112 & 112 & -22 & 192 & 602 \\
\hline $\begin{array}{l}-t \\
-5\end{array}$ & 30 & 25 & $4=$ & $29 \mathrm{k}=$ & 1 & -21 & 222 & 227 \\
\hline-5 & 37 & 40 & -36 & 77 & 78 & -20 & 53 & 437 \\
\hline-3 & 25 & 29 & -35 & 118 & 117 & -10 & 49 & 53 \\
\hline-4 & 76 & 77 & $=30$ & 73 & 81 & -18 & 109 & 108 \\
\hline-1 & 38 & 37 & -31 & 17 & mo & -16 & 29 & 27 \\
\hline 0 & 28 & 17 & -30 & 36 & 44 & -15 & 39 & 36 \\
\hline$\frac{3}{2}$ & 65 & 6.5 & -29 & 29 & 39 & -14 & 49 & 52 \\
\hline$?$ & ל3 & 53 & -28 & 48 & 49 & -13 & 60 & 63 \\
\hline 3 & 60 & 53 & -27 & 91 & 04 & -12 & 54 & 51 \\
\hline 4 & 43 & 47 & -26 & 102 & 95 & -11 & 38 & 34 \\
\hline 5 & 14 & 19 & -24 & 23 & 22 & -10 & 44 & 42 \\
\hline 8 & 25 & 29 & -27 & 117 & 122 & $4-9$ & 26 & 28 \\
\hline 9 & 74 & 64 & -22 & 34 & 29 & -5 & 44 & 32 \\
\hline 10 & 50 & 50 & -21 & 98 & 83 & -4 & 42 & 28 \\
\hline 12 & 2? & 23 & -20 & 163 & 164 & -3 & 46 & 42 \\
\hline 13 & 34 & 35 & -19 & 109 & 116 & -2 & 22 & 26 \\
\hline$r=$ & $20 k=$ & 1 & -10 & 535 & 552 & -1 & 37 & 45 \\
\hline-37 & 71 & 67 & -17 & 274 & 281 & 0 & 96 & 97 \\
\hline-36 & 51 & 57 & -16 & 178 & 182 & 2 & 114 & 114 \\
\hline-35 & 83 & 83 & -15 & 139 & 39 & 3 & 143 & 147 \\
\hline-33 & 55 & 50 & -34 & 30 & 36 & 4 & 381 & 378 \\
\hline-32 & 35 & 48 & -13 & 63 & 64 & 5 & 141 & 142 \\
\hline-31 & 201 & 201 & -11 & 30 & 23 & 6 & 42 & 45 \\
\hline-30 & 43 & 59 & O & 33 & 32 & 8 & 31 & 31 \\
\hline-25 & 71 & 76 & -8 & 37 & 36 & 49 & 125 & 126 \\
\hline-27 & 56 & 62 & - & 34 & 32 & $\mathrm{H}=$ & $31 \mathrm{k}=$ & 1 \\
\hline-2 & 64 & $5 \mathrm{G}$ & - & 54 & 57 & -36 & 477 & 75 \\
\hline$-?$ & 61 & 30 & & 61 & 66 & -32 & 122 & 131 \\
\hline- & 25 & 20 & -4 & 40 & 43 & -31 & 32 & 52 \\
\hline
\end{tabular}




\begin{tabular}{|c|c|c|c|c|c|c|c|c|c|c|}
\hline$c 5 \quad k=$ & 1 & -17 & 57 & 60 & -22 & 155 & 162 & -1 & 36 & 28 \\
\hline 173 & 173 & -31 & 51 & 55 & -21 & 108 & 105 & $\therefore 0$ & 142 & 130 \\
\hline 553 & 560 & -20 & 84 & 92 & -20 & 31 & is & 1 & $4 i$ & 27 \\
\hline 230 & 284 & -20 & 35 & 39 & -18 & 77 & 83 & ? & 79 & 29 \\
\hline 117 & 117 & -20 & $6 ?$ & 61 & -17 & 45 & 54 & 3 & 51 & 4.9 \\
\hline 47 & 250 & -27 & 35 & 79 & -15 & 117 & 119 & 1 & 57 & 63 \\
\hline 91 & 97 & -26 & 276 & 279 & -14 & 64 & 57 & 5 & 31 & 33 \\
\hline 47 & 49 & -25 & 47 & 27 & -13 & 636 & 6,39 & 5 & 68 & 64 \\
\hline $26 \quad x=$ & 12 & -24 & 43 & 48 & -12 & 176 & 34 & 8 & 34 & 30 \\
\hline 28 & 32 & $-1 ?$ & 67 & 69 & $-1 ?$ & 80 & 82 & 9 & 347 & 344 \\
\hline 30 & 92 & -21 & 24 & 57 & -20 & 19 & 22 & 10 & 30 & 40 \\
\hline 119 & 121 & -10 & 23 & 21 & -2 & 49 & 46 & $H=$ & $30 . k=$ & 1 \\
\hline 54 & 53 & -17 & 219 & 217 & -6 & 31 & 43 & -35 & 72 & 74 \\
\hline 347 & $35 \%$ & -16 & 67 & 67 & -5 & 34 & 33 & -35 & 31 & 33 \\
\hline 115 & 116 & -15 & 65 & 67 & -4 & 36 & 32 & 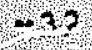 & 18 & 26 \\
\hline 24 & 199 & -14 & 204 & $4+2$ & -3 & 27 & 21 & -31 & 90 & 87 \\
\hline 52 & 34 & -13 & 30 & 40 & -1 & 21 & 7 & -28 & 41 & 45 \\
\hline 57 & 37 & -12 & 89 & 94 & 5 & 79 & $8 \mathrm{~B}$ & -37 & \pm 11 & 116 \\
\hline 74 & 25 & -11 & 109 & 105 & 6 & 64 & 59 & -25 & 52 & 71 \\
\hline 96 & $\%$ & -20 & 29 & 30 & 7 & 69 & 67 & -25 & 907 & 109 \\
\hline 271 & 275 & -9 & 147 & 150 & 9 & 86 & 85 & -34 & 438 & 27 \\
\hline 63 & 63 & -8 & 677 & 703 & 9 & 124 & 124 & -23 & 245 & 253 \\
\hline 27 & 28 & -7 & 96 & 94 & 11 & II2 & 112 & $-2 ?$ & 392 & 402 \\
\hline 28 & 27 & -6 & 30 & 25 & $\mathrm{~Hz}$ & $27 \mathrm{k}=$ & 1 & -21 & 222 & 227 \\
\hline 45 & 40 & -5 & 37 & 40 & -26 & $477 \%$ & 78 & -20 & 53 & 57 \\
\hline 37 & 47 & -3 & 25 & 23 & -35 & 118 & 117 & -19 & 49 & 35 \\
\hline 25 & 28 & -2 & 76 & 77 & -34 & 475 & 81 & -28 & 109 & 108 \\
\hline 10 & 21 & -1 & 30 & 37 & -32 & 12 & 70 & -16 & 29 & 27 \\
\hline 115 & 114 & 0 & 28 & 17 & -30 & 36 & 44 & -15 & 39 & 36 \\
\hline 246 & 237 & 1 & 65 & 65 & -29 & 29 & $=7$ & -14 & 49 & 52 \\
\hline 46 & 48 & 2 & 53 & 53 & -28 & 48 & 49 & -13 & 60 & 63 \\
\hline 68 & 70 & 3 & 60 & 59 & -27 & 191 & 94 & -12 & 54 & 51 \\
\hline 63 & 63 & 4 & 43 & 47 & -26 & 102 & 95 & -11 & 38 & 34 \\
\hline 72 & 70 & 5 & 14 & $2 \mathrm{~A}$ & -24 & 23 & 22 & -10 & 44 & 42 \\
\hline 165 & 163 & 8 & 25 & 29 & $-? 3$ & 127 & 122 & $18-9$ & 26 & 28 \\
\hline 131 & 129 & 9 & 74 & 64 & -22 & 34 & 29 & -5 & 44 & 32 \\
\hline 309 & 311 & 10 & 58 & 50 & -21 & 89 & 89 & -4 & 42 & 28 \\
\hline 484 & 497 & 12 & $2 ?$ & 23 & -20 & 163 & 164 & -3 & 46 & 42 \\
\hline 134 & 139 & 13 & 34 & 35 & -19 & 109 & 116 & -2 & 23 & 26 \\
\hline 15 & 2 & $r t=$ & $28 \mathrm{c}=$ & 11 & -18 & 535 & 352 & -1 & 37 & 45 \\
\hline 58 & 57 & -37 & 71 & 67 & -17 & 274 & $2 A 1$ & 0 & 98 & 97 \\
\hline 33 & 35 & -36 & 51 & 57 & -16 & 178 & 182 & 2 & 114 & 114 \\
\hline 35 & 33 & -35 & 83 & 83 & -15 & 38 & 39 & 3 & 143 & 147 \\
\hline 22 & 41 & -33 & 55 & 59 & -14 & 30 & 36 & 4 & 381 & 372 \\
\hline 36 & 39 & -32 & 35 & 48 & -13 & 63 & 64 & 5 & 141 & 142 \\
\hline 43 & 46 & -31 & $20 !$ & 201 & 41 & $3 n$ & 26 & 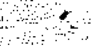 & 43 & 45 \\
\hline 65 & 53 & -30 & 63 & 58 & -3 & 93 & 32 & 9 & 31 & 31 \\
\hline $27 x=$ & 1 & -29 & 71 & 75 & -8 & 37 & 36 & 8 & 125 & 126 \\
\hline 45 & 48 & -27 & 56 & 62 & -7 & 34 & 32 & $H:=$ & $31 x=$ & 1 \\
\hline 26.1 & 262 & -26 & 64 & 56 & -6 & 54 & 57 & -36 & 877 & 75 \\
\hline $77 ?$ & 72 & -24 & 61 & 30 & -5 & 61 & 66 & -32 & 122 & 131 \\
\hline 100 & 106 & -23 & 26 & 20 & -4 & 40 & 43 & -31 & 42 & 52 \\
\hline
\end{tabular}


$\mathrm{L} F \mathrm{FO}$ FC C L FO FC, FO FC L FO FC

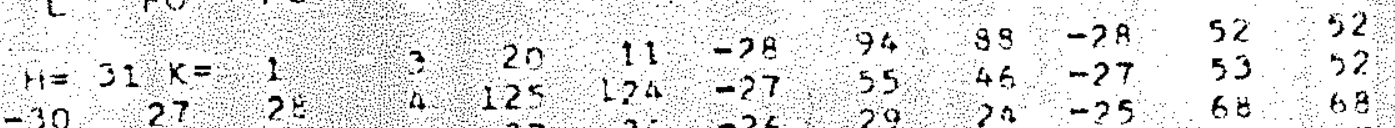

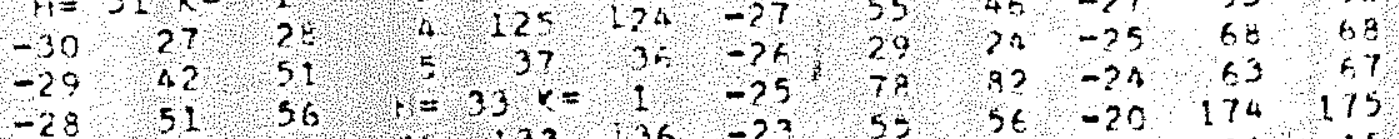

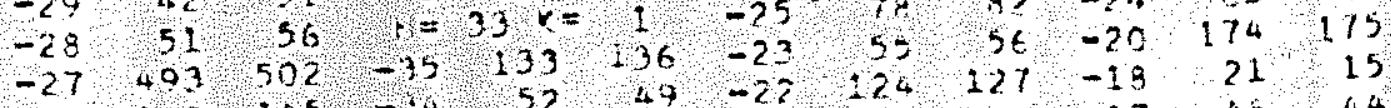

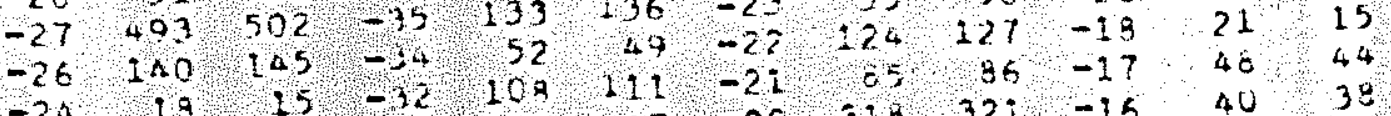

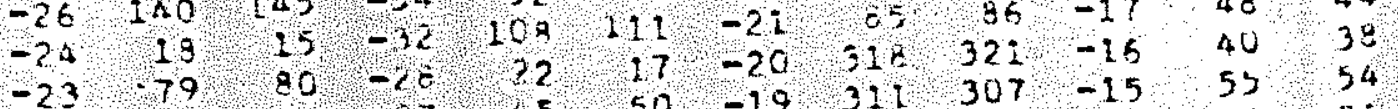

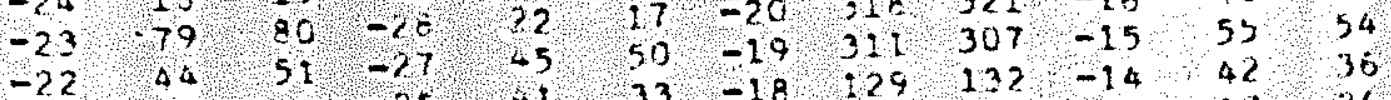

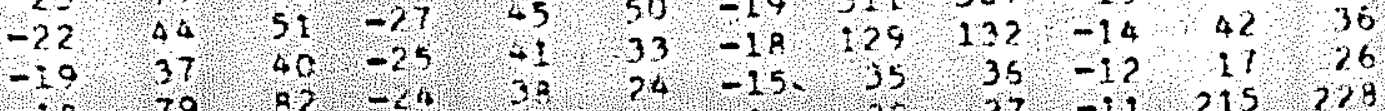

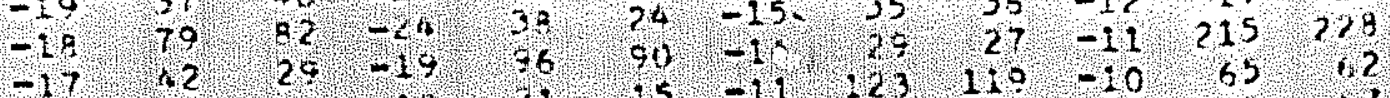

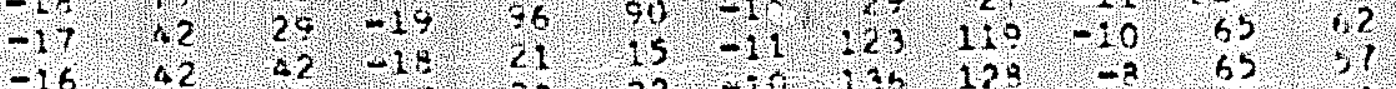

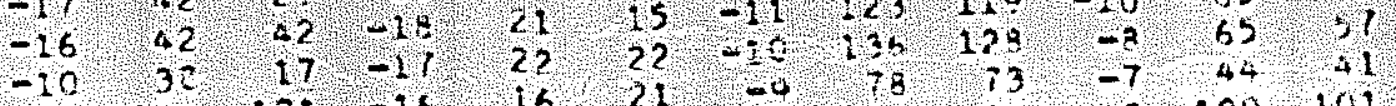

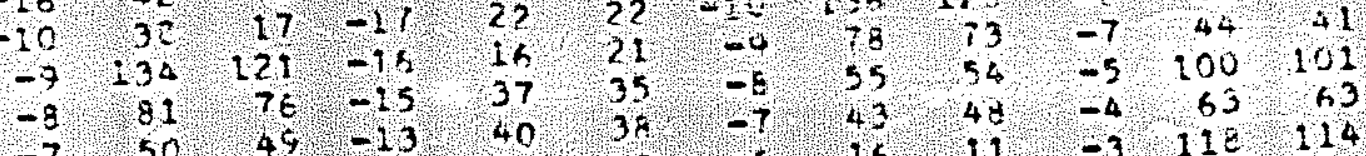

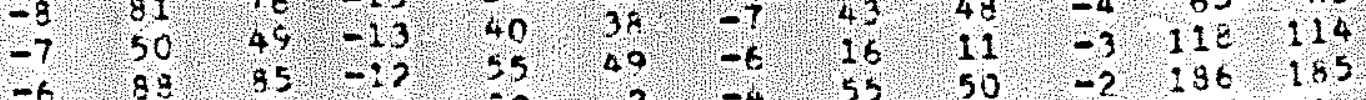

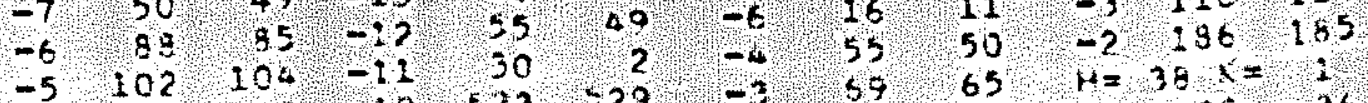

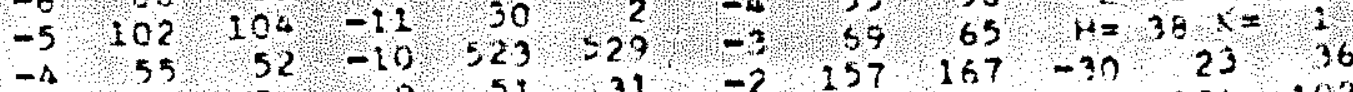

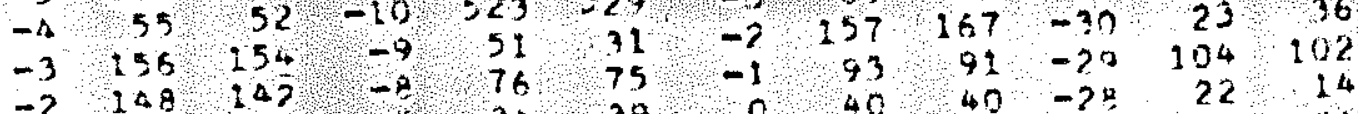

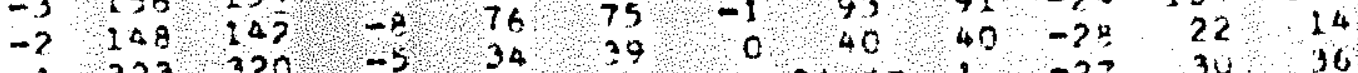

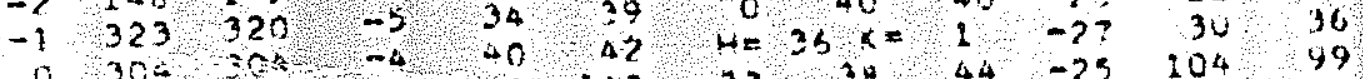

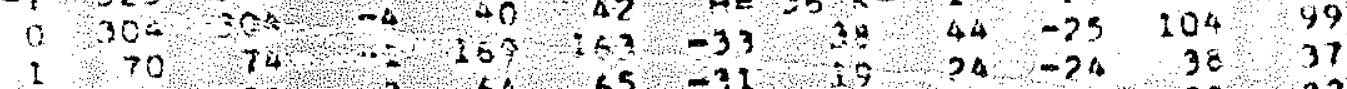

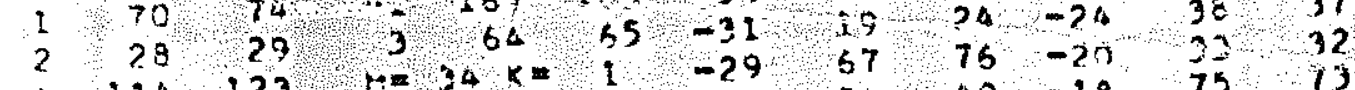

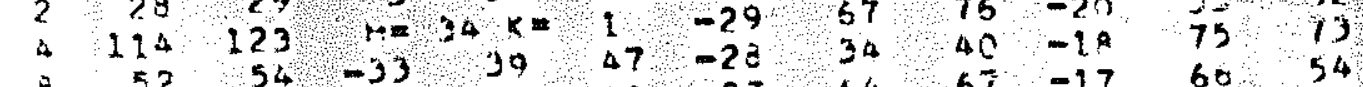

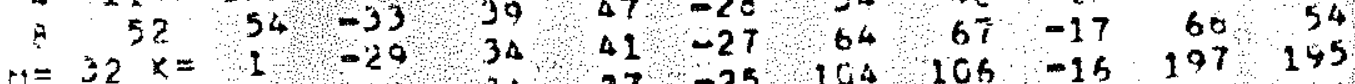

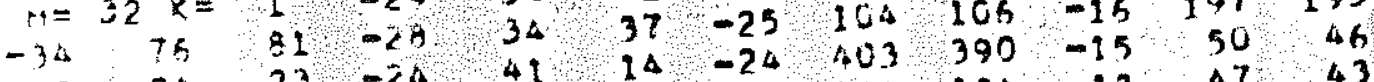

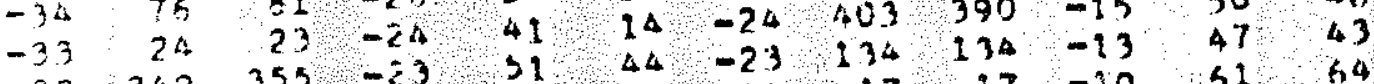

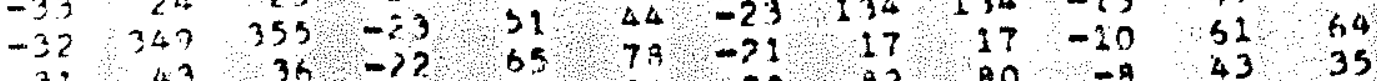

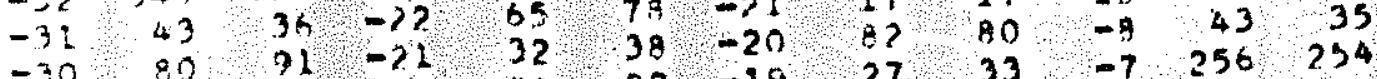

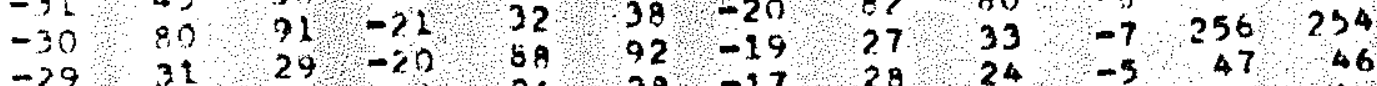

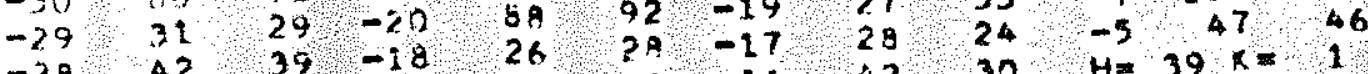

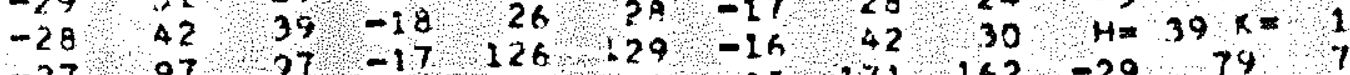

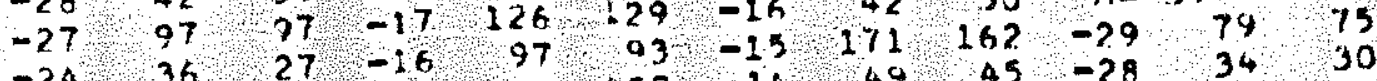

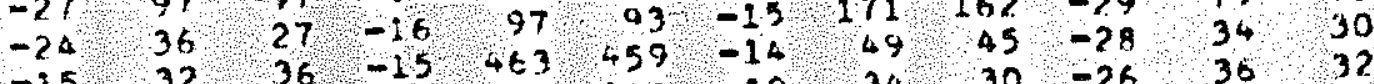

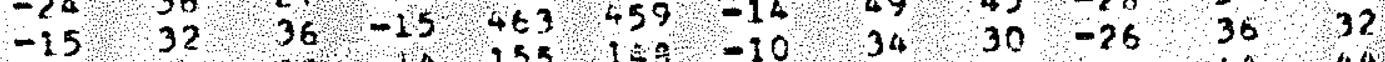

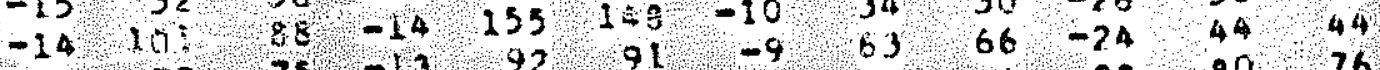

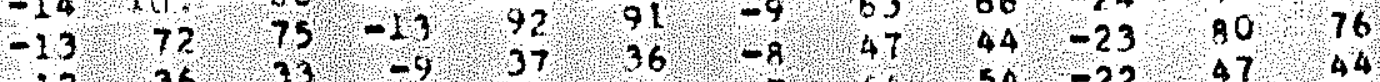

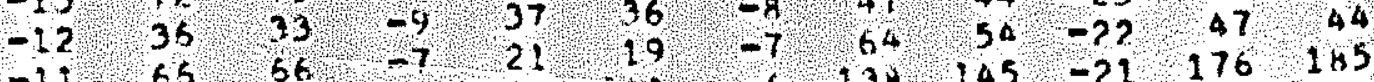

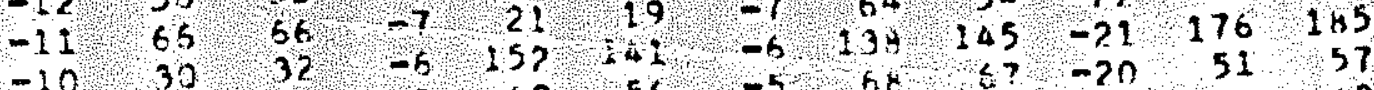

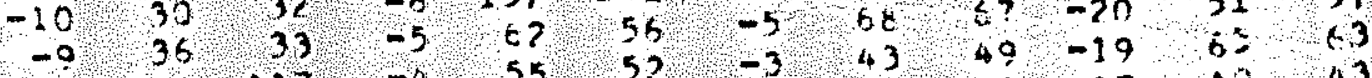

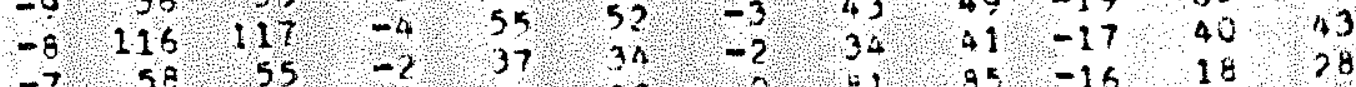

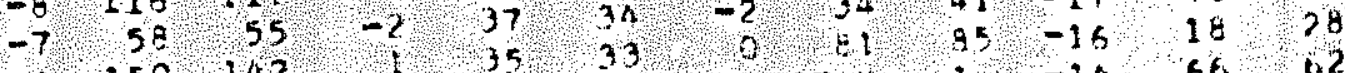

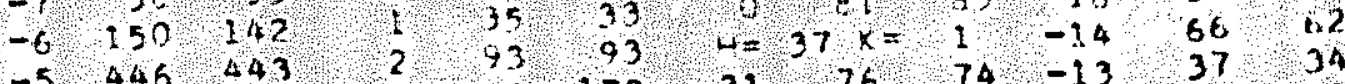

$\begin{array}{llllllllllll}-5 & 446 & 443 & 2 & 163 & 170 & -31 & 76 & 74 & -13 & 37 & 34\end{array}$

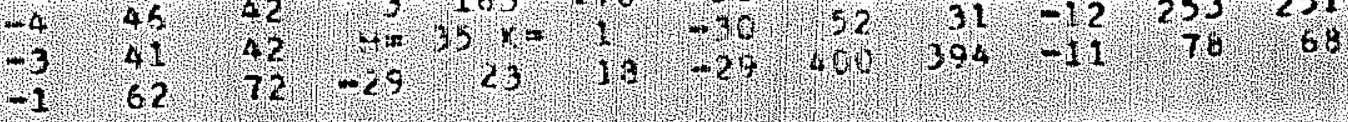




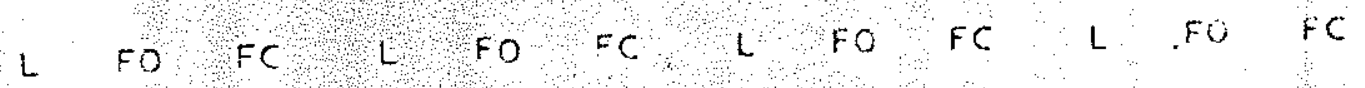

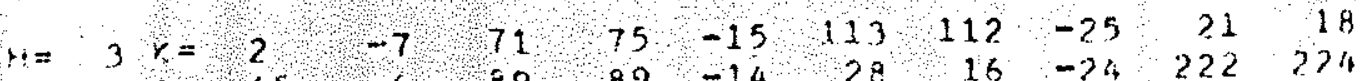

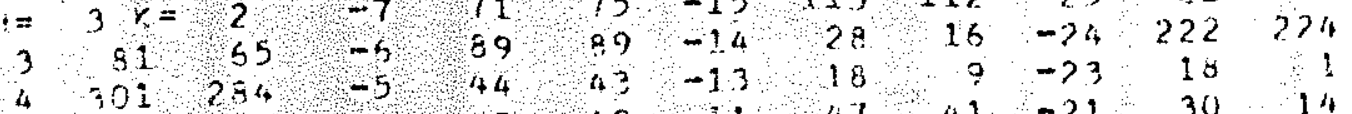

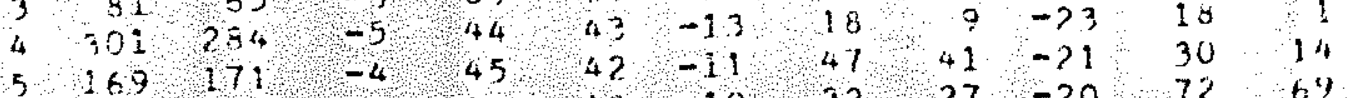

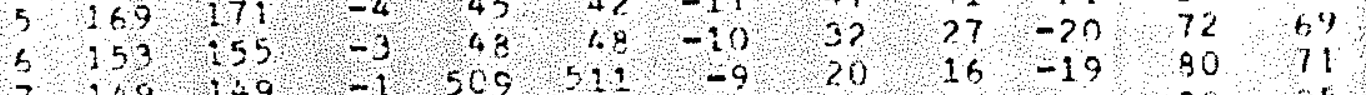

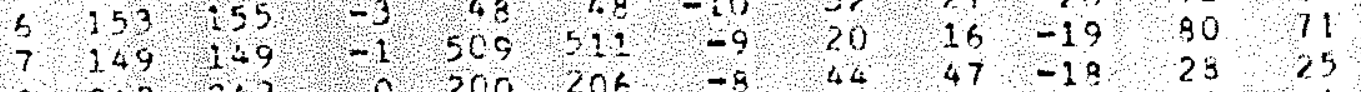

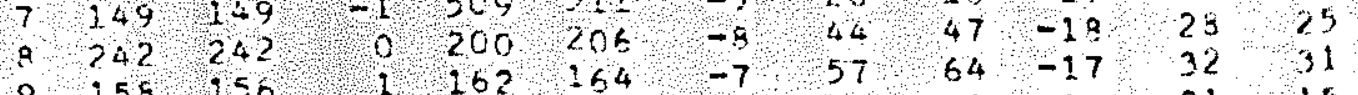

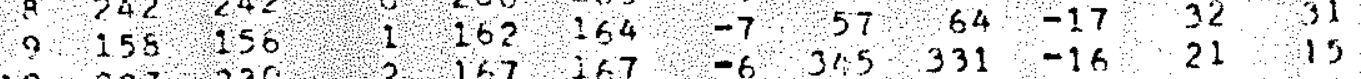

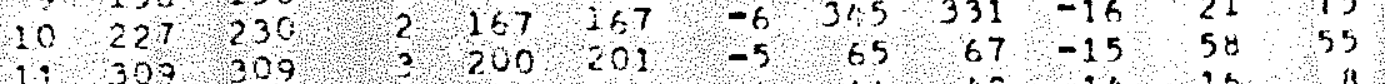

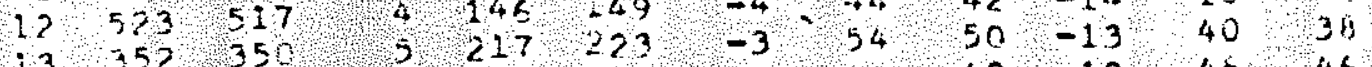

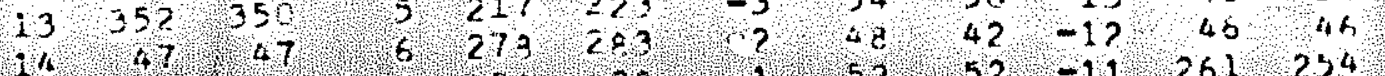

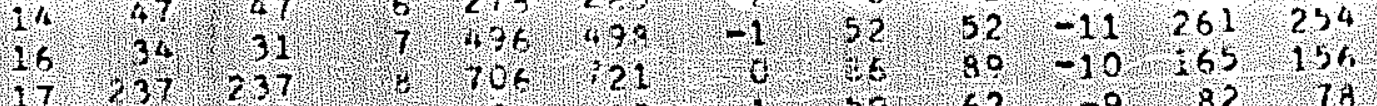

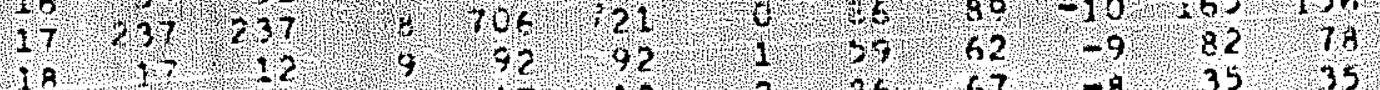

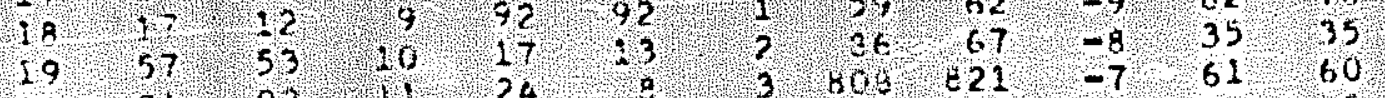

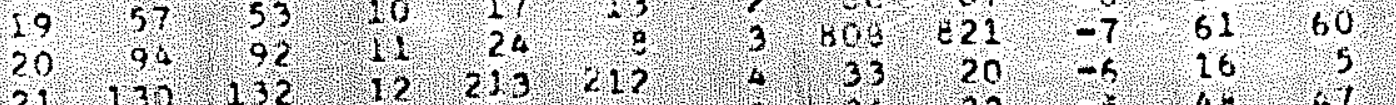

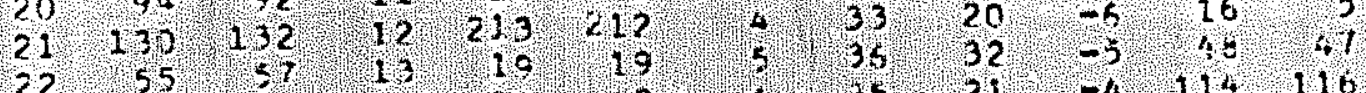

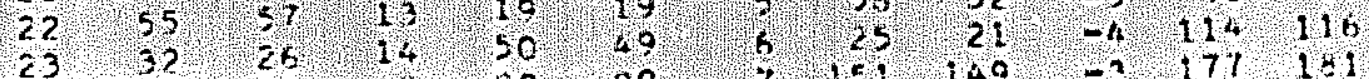

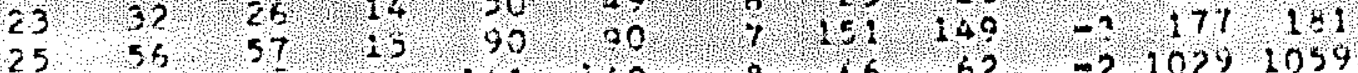

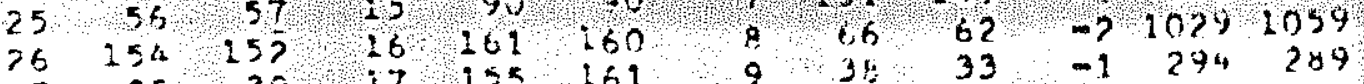

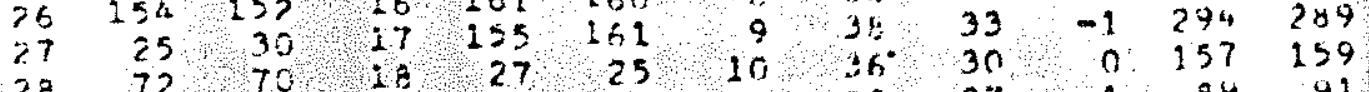

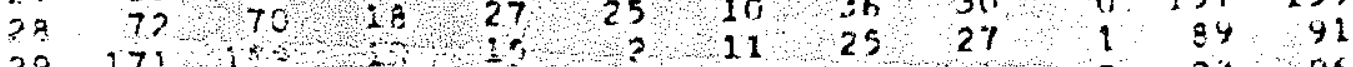

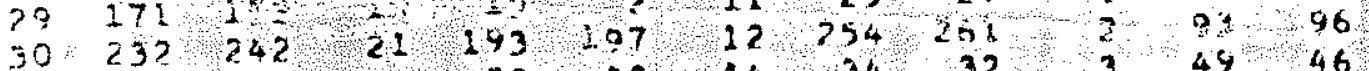

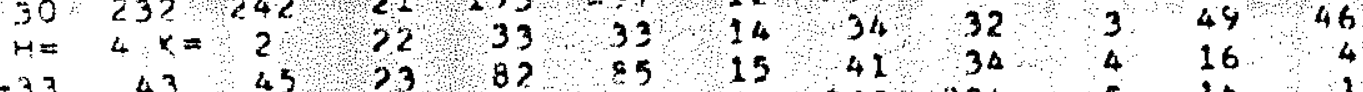

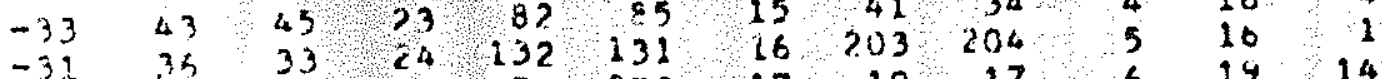

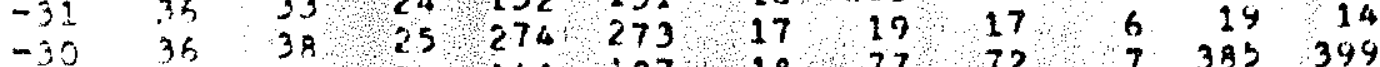

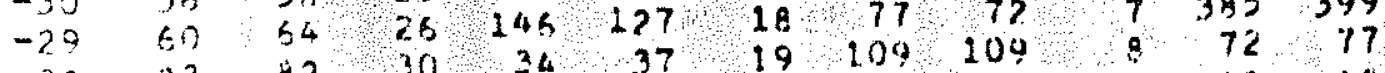

$\begin{array}{lll}-29 & 0\end{array}$

$\begin{array}{lll}-27 & 29 & 23 \\ -26 & 39 & 35\end{array}$

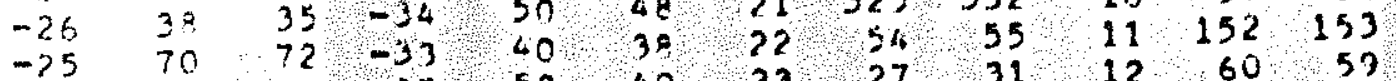

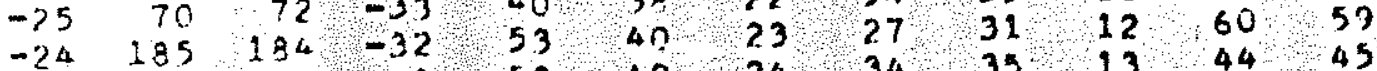

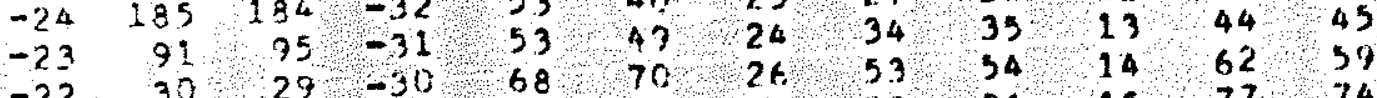

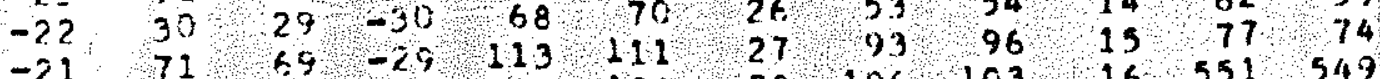

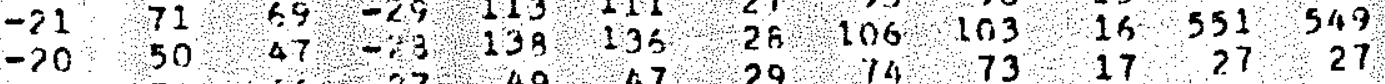

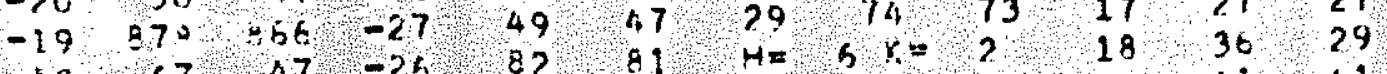

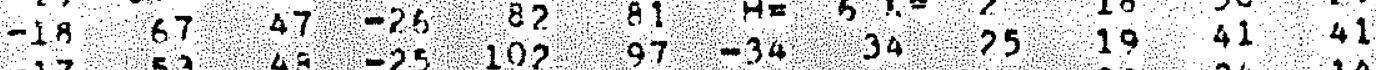

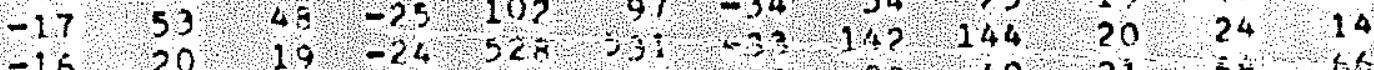

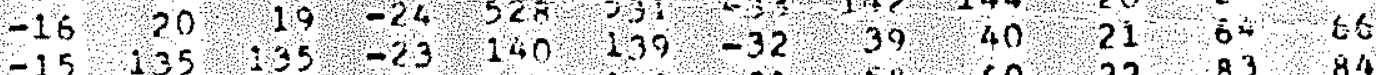

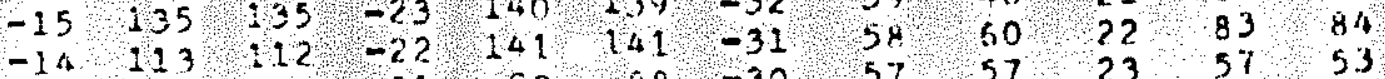

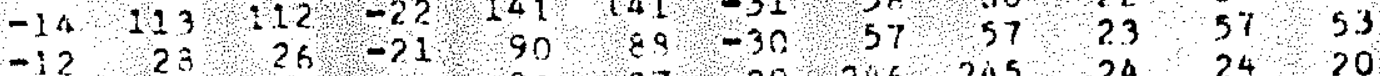

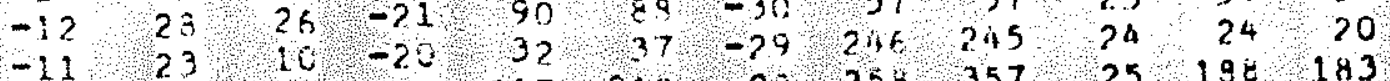

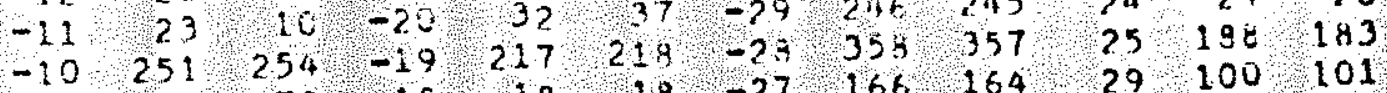

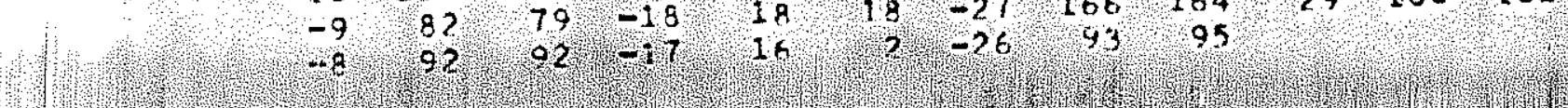


$\mathrm{L} F \mathrm{FO} F \mathrm{FC}$ L $\mathrm{FO} F \mathrm{FC}$ L FO FC L FO FC

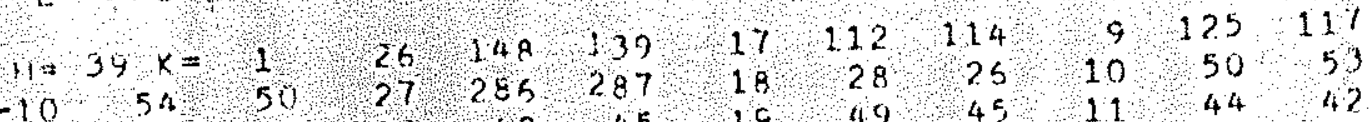

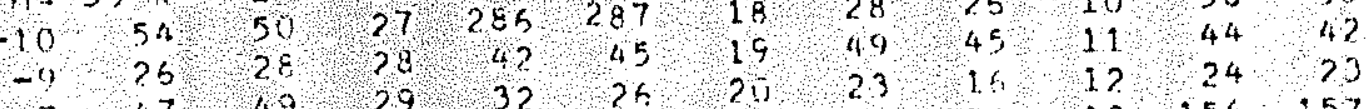

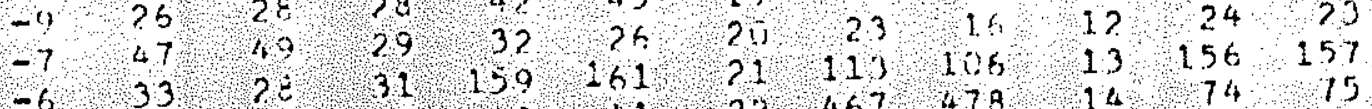

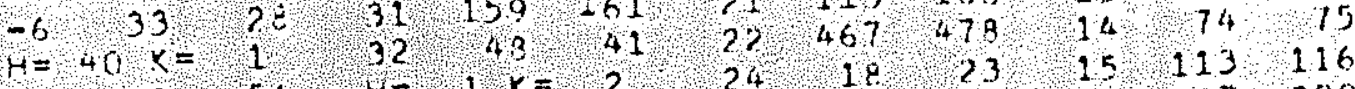

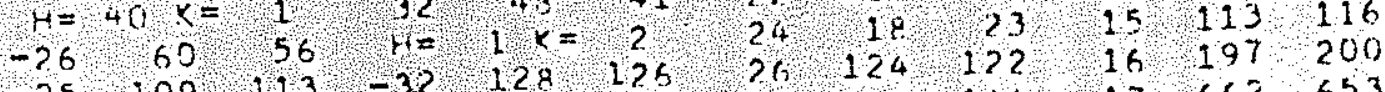

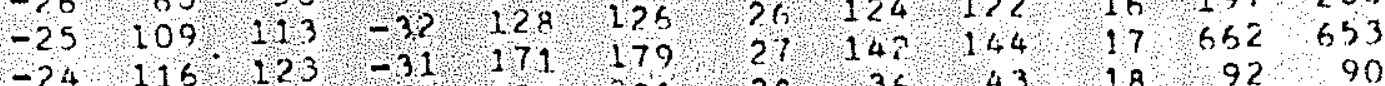

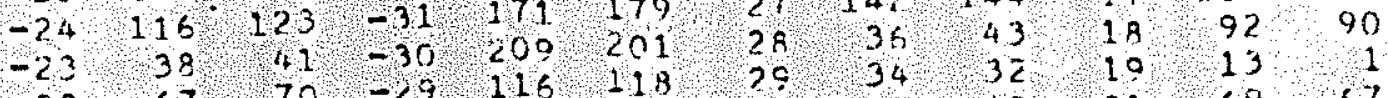

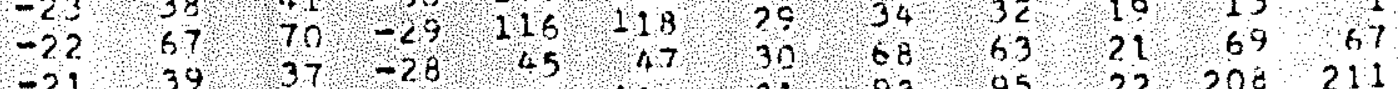

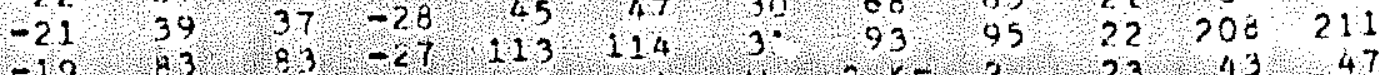

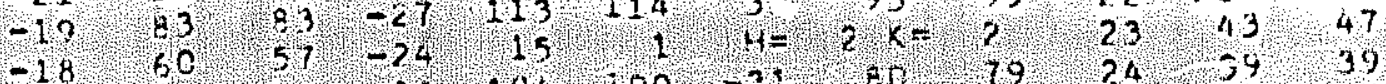

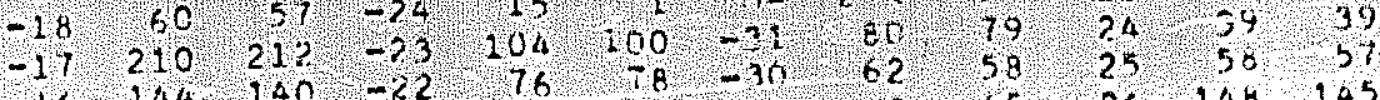

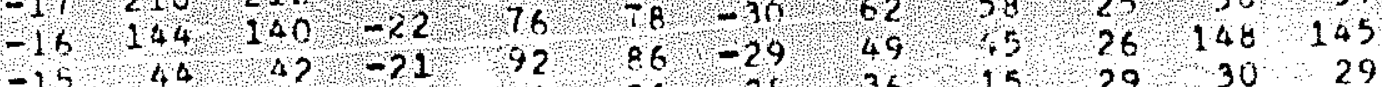

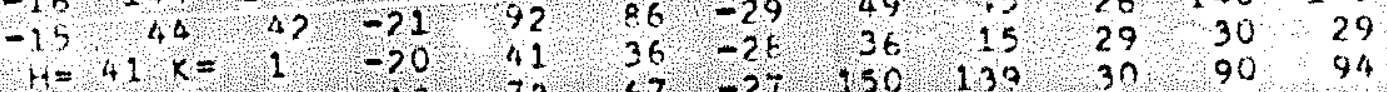

$\begin{array}{llllllllllll}-24 & 50 & 61 & -16 & 73 & 67 & -27 & 150 & 139 & 30 & 90 & 94\end{array}$

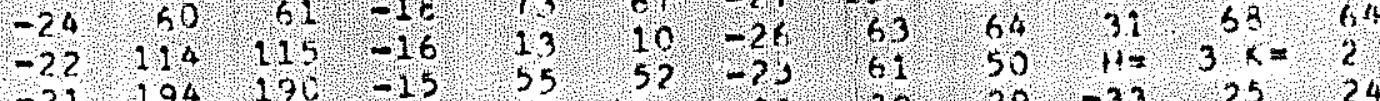

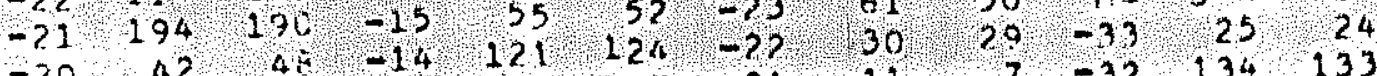

$-20 \quad 42 \quad 46-140140 \quad 37-21 \quad 11 \quad 7 \quad-32 \quad 134,133$

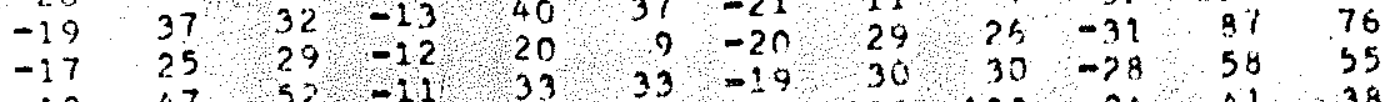

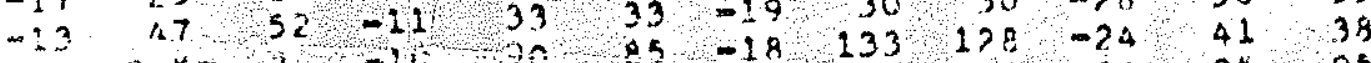

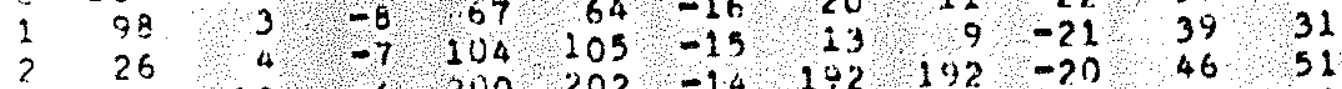

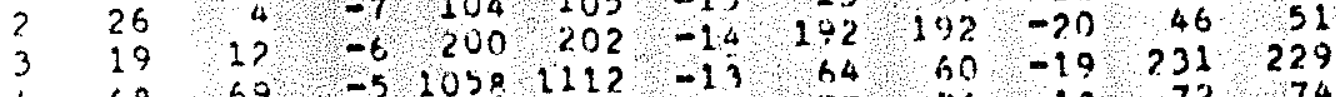

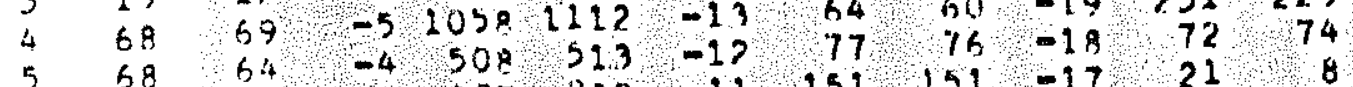

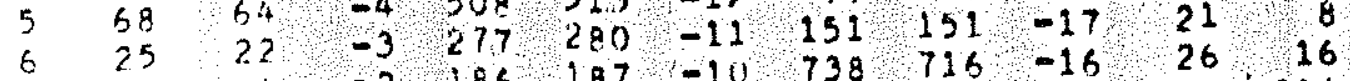

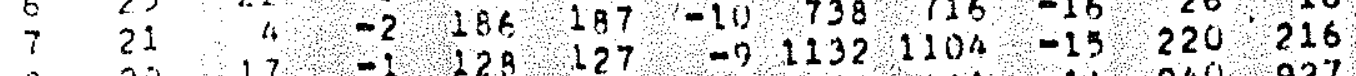

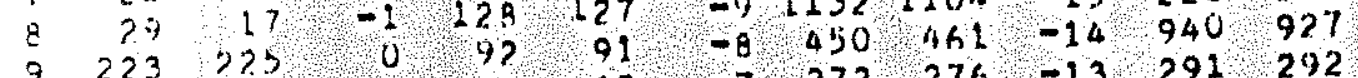

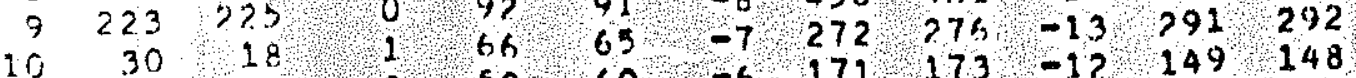

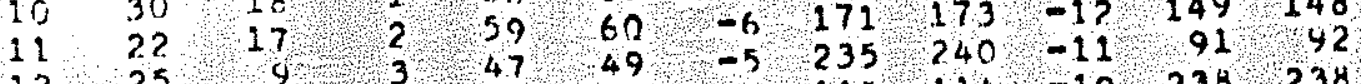

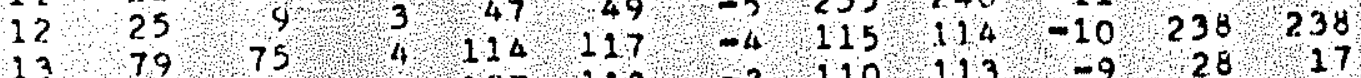

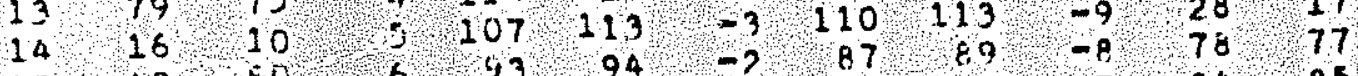

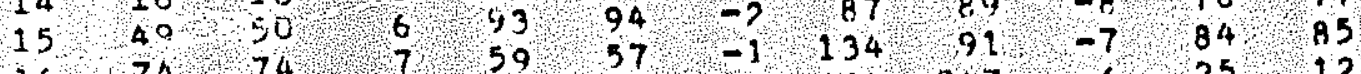

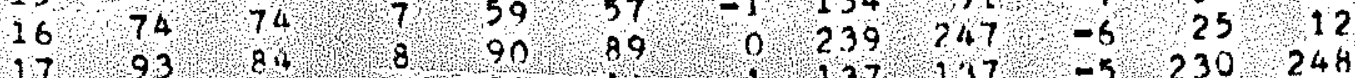

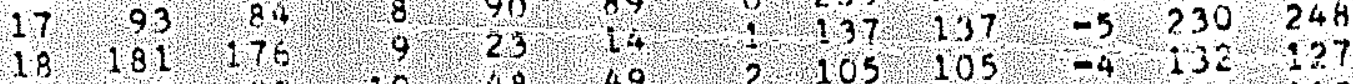

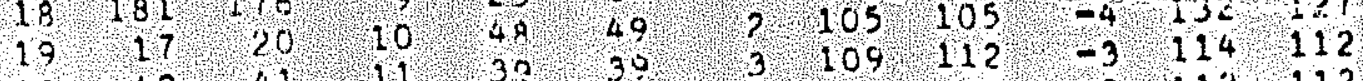

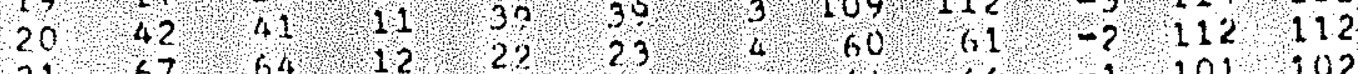

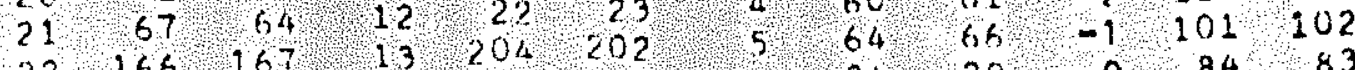

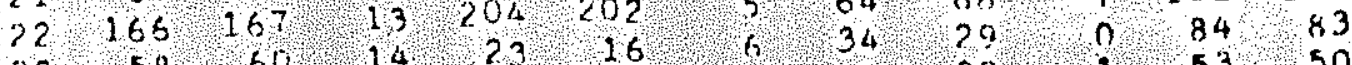

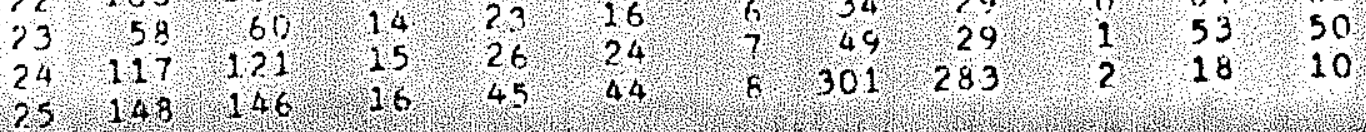




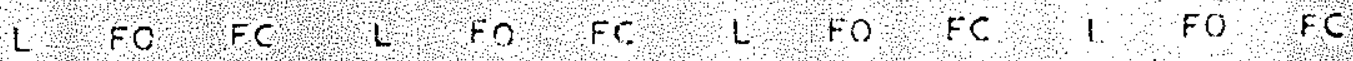

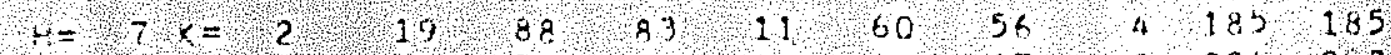

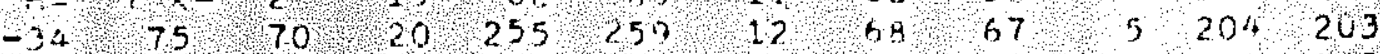

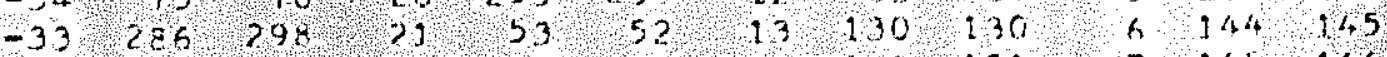

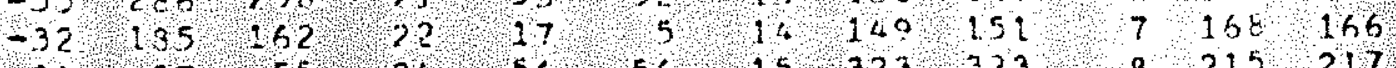

$\begin{array}{lllllllllll}-31 & 57 & 55 & 24 & 56 & 56 & 15 & 323 & 323 & 9 & 215 \\ -30 & 20 & 5 & 25 & 114 & -11 & 16 & 115 & 112 & 210\end{array}$

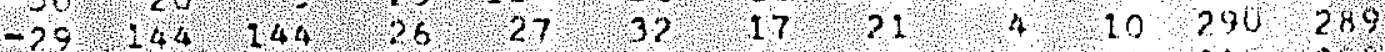

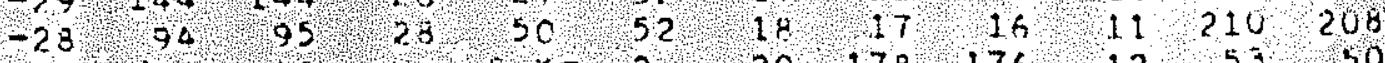

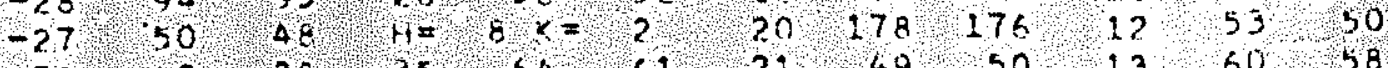

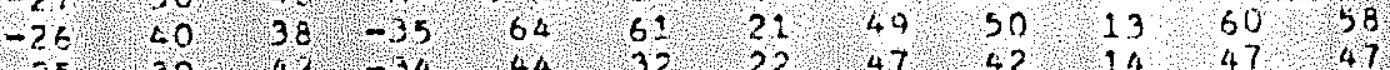

$\begin{array}{lllllllllllll}-75 & 39 & 42 & -34 & 44 & 37 & 22 & 47 & 42 & 14 & 47 \\ -24 & 21 & 19 & -33 & 157 & 160 & 23 & 70 & 72 & 15 & 182\end{array}$

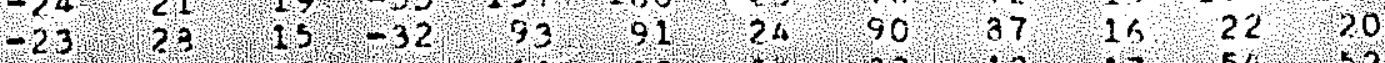

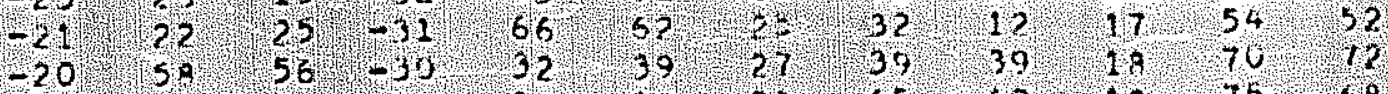

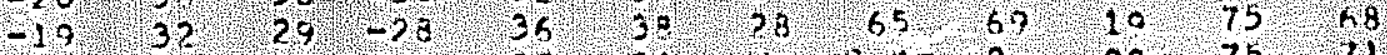

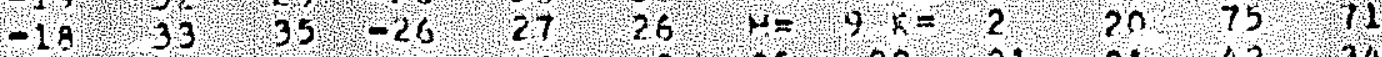

$\begin{array}{lllllllllllllll}-17 & 26 & 27 & -25 & 45 & 43 & -15 & 27 & 21 & 21 & 42\end{array} \mathbf{3}^{4}$

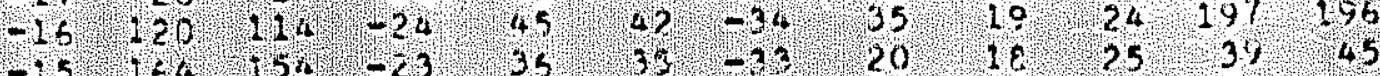

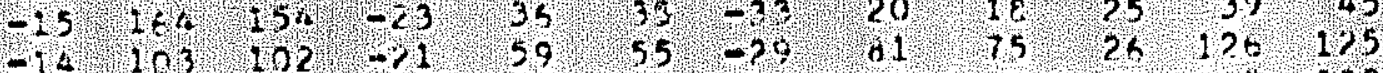

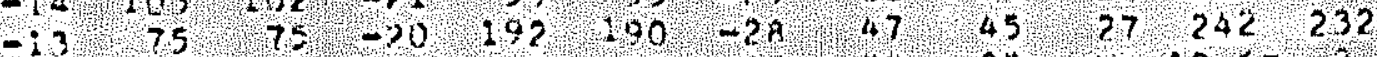

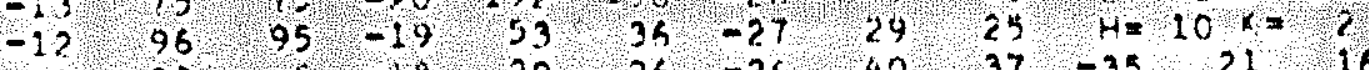

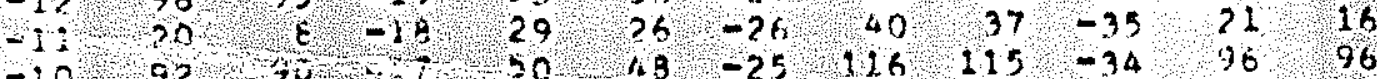

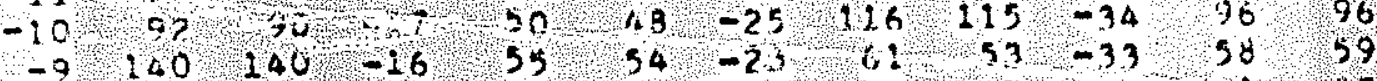

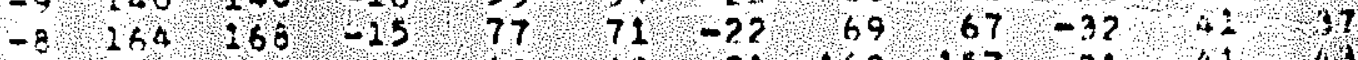

$\begin{array}{lllllllllll}-7 & 629 & 638 & -14 & 68 & 69 & -21 & 150 & 157 & -31 & 41\end{array}$

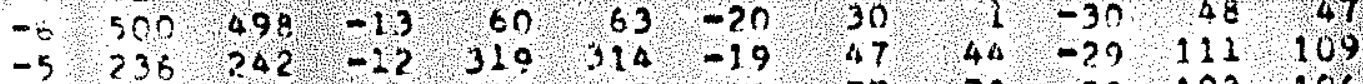

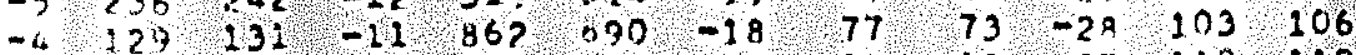

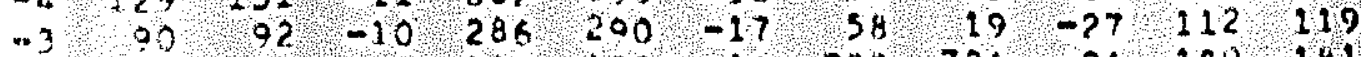

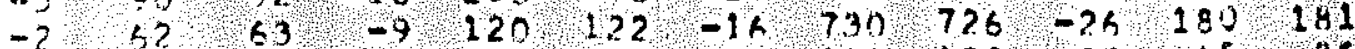

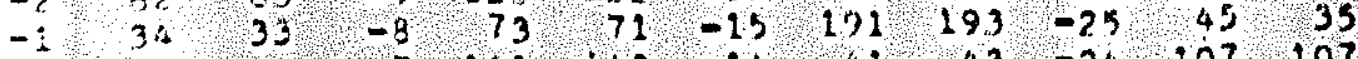

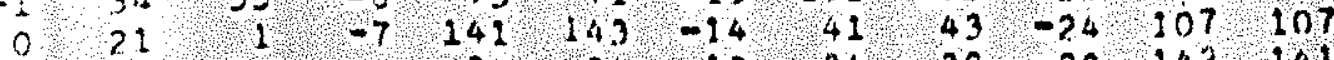

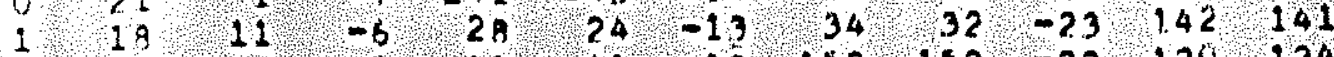

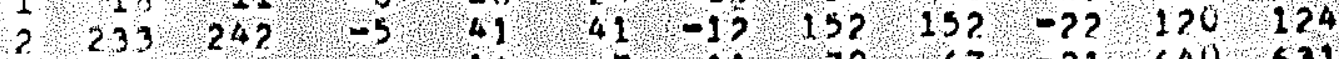

$\begin{array}{llllllllll}3 & 241 & 250 & -4 & 16 & 7 & -11 & 70 & 67 & -21 \\ 4 & 640,631\end{array}$

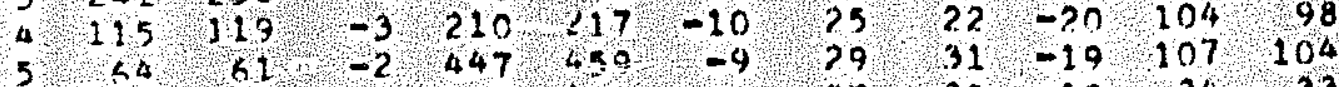

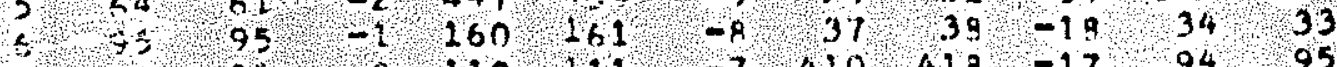

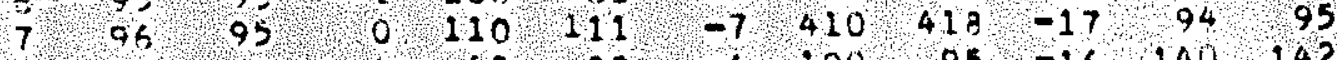

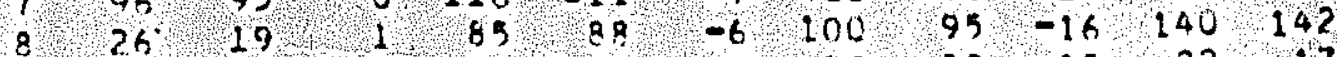

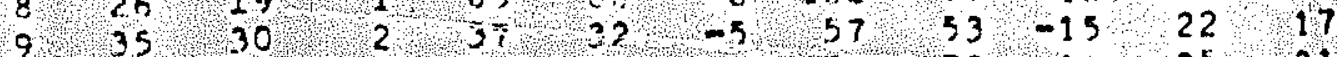

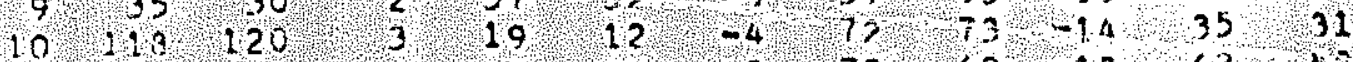

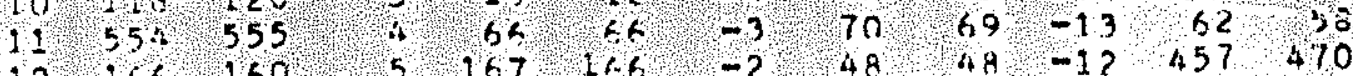

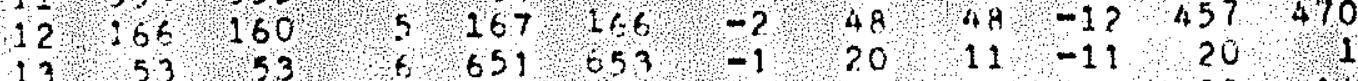

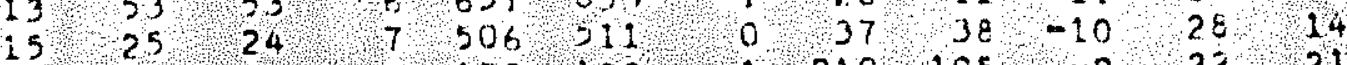

$\left.\begin{array}{llllllllllllll}16 & 34 & 36 & 8 & 190 & 192 & 1 & 210 & 195 & -0 & 22\end{array}\right)$

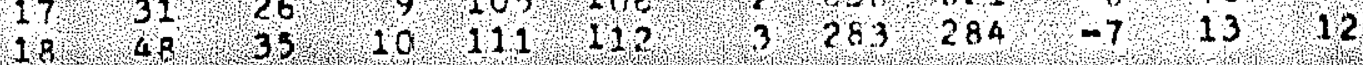




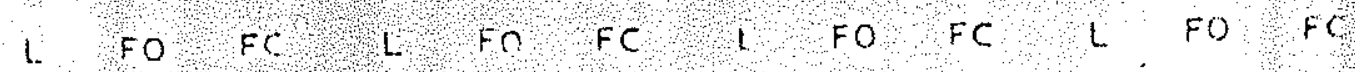

\begin{tabular}{|c|c|c|c|c|c|c|c|c|c|c|c|}
\hline $11=$ & $16 k=$ & 2 & -15 & 39 & 34 & -20 & 68. & 67 & -23 & 11 & 13 \\
\hline-6 & 33 & 35 & -14 & 22 & 16 & -19 & 20 & 1.6 & -22 & 131 & 128 \\
\hline-5 & 87 & 37 & -13 & $5 \%$ & 67 & -18 & 44 & $<1$ & -21 & 125 & 125 \\
\hline 4 & 53 & 59 & -12 & 106 & 103 & -17 & 121 & 121 & -20 & 89 & 91 \\
\hline 3 & 1137 & 11,5 & -11 & 40 & 47 & -16 & 47 & 74 & -19 & 41 & 42 \\
\hline & 131 & $13 \%$ & -10 & 115 & 115 & -15 & 105 & 107 & -18 & 179 & 178 \\
\hline & 100 & 89 & -9 & 142 & 1,5 & -14 & 104 & 104 & -17 & 554 & 557 \\
\hline 1 & 131 & 130 & -3 & 705 & 749 & -13 & 485 & 481 & -16 & 156 & 155 \\
\hline 1 & 205 & 102 & -1 & 270 & 260 & -12 & 687 & 694 & -15 & 46 & 36 \\
\hline 2 & 128 & 120 & -6 & 176 & 173 & -11 & 294 & 290 & -16 & 40 & 29 \\
\hline 4 & 186 & 181 & -5 & 81 & 81 & -10 & 149 & 149 & -13 & 31 & 3 \\
\hline 5 & 159 & 156 & -4 & 80 & 83 & -9 & 132 & 131 & -11 & 74 & 73 \\
\hline 5 & 302 & 198 & -3 & 45 & 47 & -5 & 114 & 111 & -10 & 97 & 97 \\
\hline & 392 & 389 & -2 & 16 & 9 & $=7$ & 82 & 79 & -9 & 230 & 278 \\
\hline & 67 & 52 & 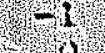 & 40 & $7^{3} 5$ & -4 & 149 & 147 & 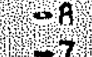 & $\begin{array}{r}404 \\
12\end{array}$ & 993 \\
\hline 9 & 61 & 79 & 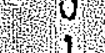 & $\begin{array}{l}47 \\
50 \%\end{array}$ & 86 & (r) & $\begin{array}{r}179 \\
537\end{array}$ & $\begin{array}{l}179 \\
531\end{array}$ & -7 & $\begin{array}{r}110 \\
28\end{array}$ & 211 \\
\hline & 157 & $\begin{array}{r}59 \\
151\end{array}$ & 2 & 509 & $\begin{array}{r}497 \\
93\end{array}$ & -4 & 258 & 35 & -5 & 13 & 10 \\
\hline & 27 & 25 & 3 & 99 & $\begin{array}{l}93 \\
38\end{array}$ & $=2$ & 93 & 095 & -3 & 15 & 10 \\
\hline $1 \% 3$ & 24 & 22 & 4 & 31 & 28 & -1 & 20 & 20 & -2 & 19 & 18 \\
\hline 13 & 51 & 43 & 5 & 19 & 77 & 0 & 15 & 10 & -1 & 16 & \\
\hline 14 & 42 & 41 & 6 & 53 & 53 & 1 & 60 & 61 & 0 & 112 & 114 \\
\hline 15 & 140 & $14 \%$ & 7 & 21 & 17 & 4 & 19 & 16 & 1 & 223 & 275 \\
\hline 17 & 34 & 34 & 9 & 16 & 5 & 5 & 174 & 175 & 2 & 61 & 58 \\
\hline 19 & 59 & 58 & 10 & 165 & 165 & 6 & 159 & 161 & 3 & 14 & 8 \\
\hline $\begin{array}{l}19 \\
20\end{array}$ & 234 & 29 & 18 & 415 & 76 & 7 & 104 & 102 & 5 & 163 & 153 \\
\hline $\begin{array}{l}20 \\
21\end{array}$ & 38 & 435 & 12 & 102 & 002 & A & 49 & 83 & 6 & 35 & 31 \\
\hline 21 & 220 & 120 & 13 & 101 & 99 & 9 & 125 & 123 & 7 & 126 & 178 \\
\hline 6 & 167 & 161 & 14 & 213 & 214 & 10 & 111 & 111 & 8 & $\begin{array}{l}202 \\
368\end{array}$ & 206 \\
\hline & 139 & 130 & 15 & 39 & 39 & 11 & 18 & 45 & 10 & $\begin{array}{l}358 \\
378\end{array}$ & 362 \\
\hline $\begin{array}{l}25 \\
4=\end{array}$ & $\begin{array}{r}759 \\
34\end{array}$ & 260 & 16 & 78 & $\begin{array}{l}76 \\
50\end{array}$ & 13 & & 141 & 11 & 122 & 126 \\
\hline $4=$ & $11^{34} k=$ & 2 & 18 & 111 & 103 & 14 & 548 & 546 & 12 & 42 & 42 \\
\hline 4 & 110 & 111 & 29 & 415 & 422 & 15 & 126 & 184 & 13 & 80 & 01 \\
\hline 32 & 115 & 110 & (23 & 174 & 669 & 16 & 45 & 50 & 14 & 80 & 88 \\
\hline 32 & 491 & 96 & 24 & 100 & 203 & 17 & 23 & 17 & 15 & 34 & 34 \\
\hline & 93 & 100 & 23 & 57 & 61 & 18 & 63 & 113 & 16 & $\begin{array}{l}42 \\
28\end{array}$ & $\begin{array}{l}44 \\
20\end{array}$ \\
\hline & $\begin{array}{l}41 \\
67\end{array}$ & $\begin{array}{r}35 \\
67\end{array}$ & 26 & $12 \%$ & 39 & $\begin{array}{l}10 \\
20\end{array}$ & 42 & 41 & 18 & 102 & 102 \\
\hline & 94 & 96 & -35 & 790 & 92 & 23 & 41 & 33 & 20 & 36 & 32 \\
\hline & 99 & 99 & .33 & 27 & 23 & $\mathrm{H}=$ & $13 \mathrm{~K}=$ & 2 & 21 & 33 & 2 \\
\hline & 364 & 352 & -11 & 127 & 123 & $\begin{array}{r}-35 \\
-34\end{array}$ & 1262 & 261 & 22 & $\begin{array}{l}31 \\
35\end{array}$ & 8 \\
\hline 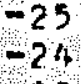 & $\begin{array}{l}160 \\
179\end{array}$ & 167 & $\begin{array}{r}-30 \\
-20\end{array}$ & $32 \%$ & $\begin{array}{r}16 \\
150\end{array}$ & $\begin{array}{r}-34 \\
-33\end{array}$ & 147 & $\begin{array}{r}135 \\
19\end{array}$ & 23 & $14 \mathrm{~K}=$ & 2 \\
\hline & $\begin{array}{r}179 \\
62\end{array}$ & 177 & $\begin{array}{r}-29 \\
-29\end{array}$ & 20 & 26 & $-3 ?$ & $\begin{array}{r}21 \\
39\end{array}$ & $\begin{array}{r}19 \\
35\end{array}$ & -36 & 438 & 38 \\
\hline & 6 & 89 & $-<7$ & 20 & 29 & -31 & 110 & 105 & -35 & 152 & 14 \\
\hline & 184 & 183 & -26 & 156 & 156 & $-3 n$ & 143 & 139 & 34 & 053 & \\
\hline & 59 & 60 & -25 & 195 & 09 & -20 & 57 & 54 & -31 & $10 \%$ & 10 \\
\hline & 63 & 64 & -24 & 73 & 74 & -28 & 45 & 33 & -3 & 40 & \\
\hline & 82 & $7 \%$ & -23 & 24 & 80 & -27 & 39 & 134 & -2 & 43 & \\
\hline & 259 & 264 & -22 & 135 & 137 & -26 & 131 & 133 & -28 & 32 & \\
\hline & 178 & 79 & -71 & 205 & 204 & -25 & 36 & 27 & & 83 & \\
\hline
\end{tabular}


FO $F C$ L $F O F F$ L FO FC L FO FC

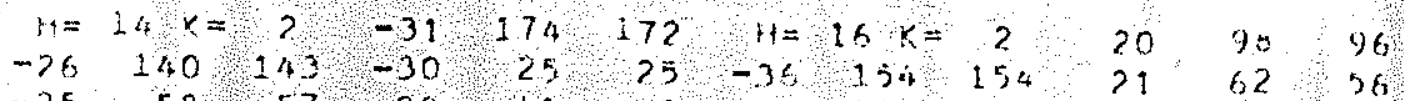

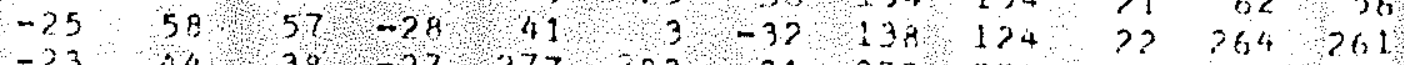

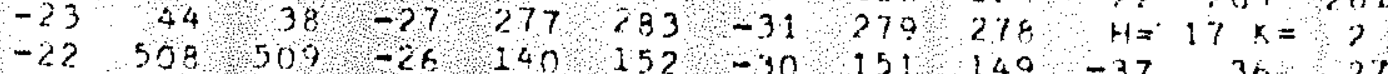

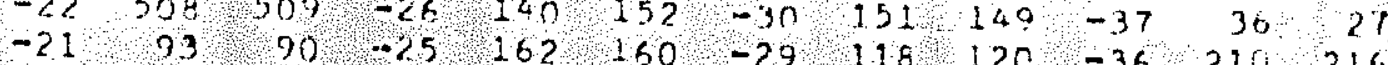

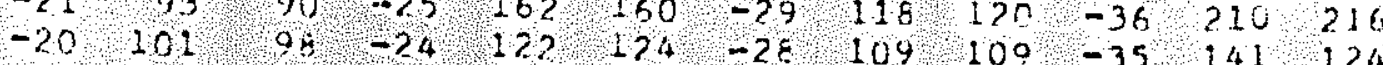

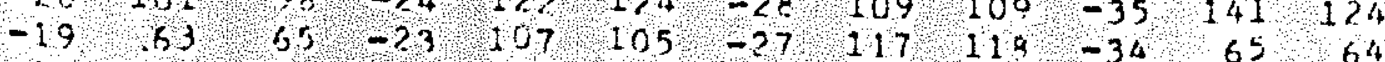

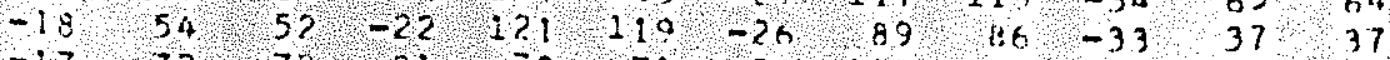

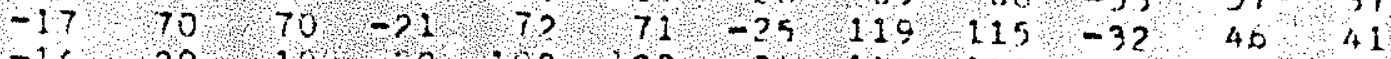

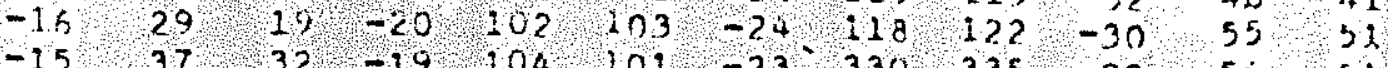

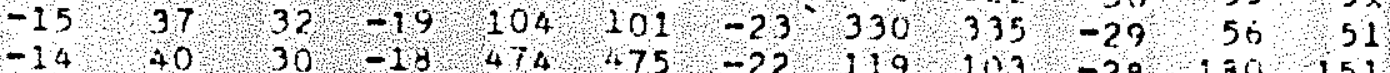

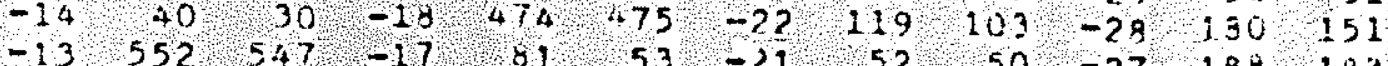

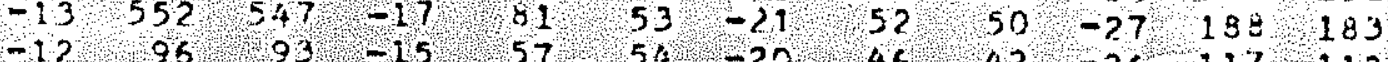

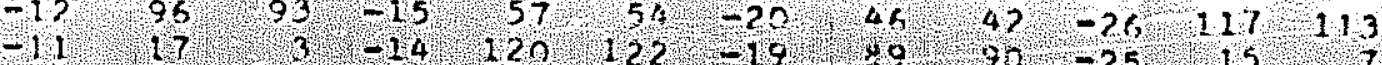

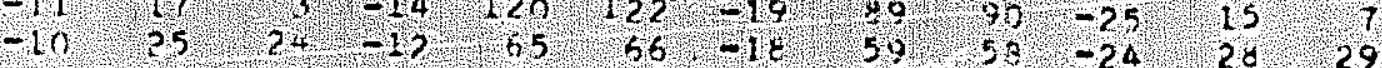

$\begin{array}{llllllllllll}-9 & 81 & 9 & -11 & 76 & 77 & -17 & 67 & 65 & -23 & 100 & 99\end{array}$

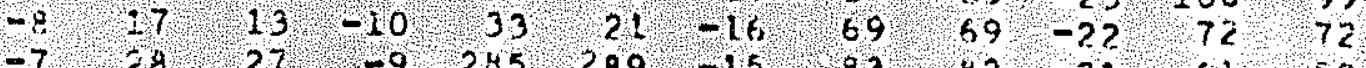

$\begin{array}{lllllllllllll}-7 & 29 & 27 & -9 & 285 & 299 & -15 & 83 & 42 & -21 & 61 & 99\end{array}$

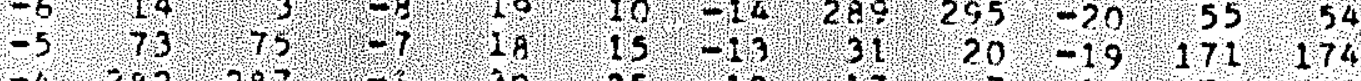

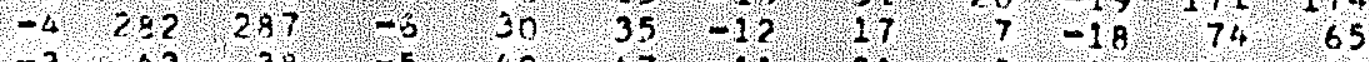

$\begin{array}{llllllllllll}-3 & 42 & 38 & -5 & 40 & 47 & -11 & 21 & 9 & -16 & 38 & 40\end{array}$

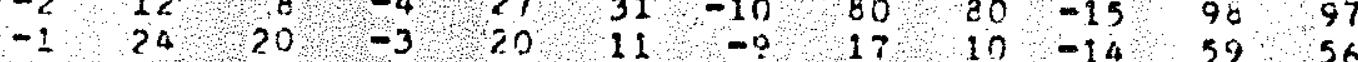

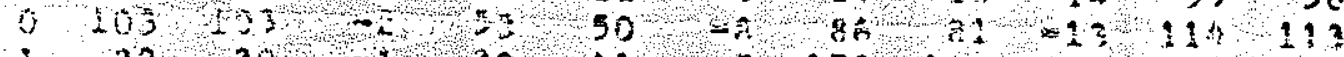

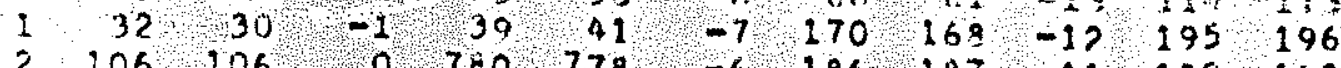

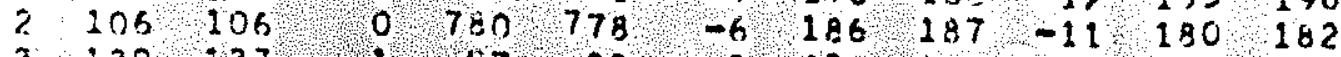

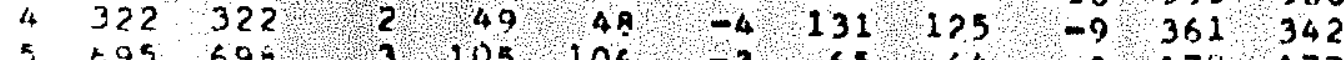

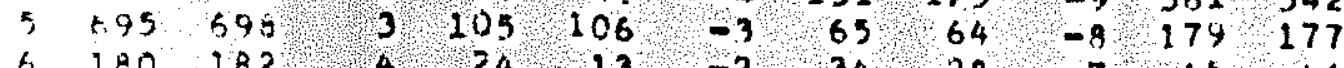

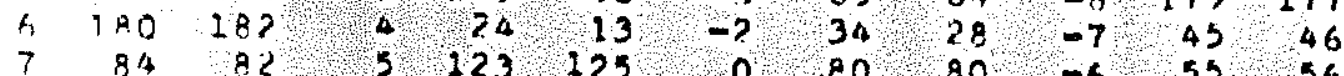

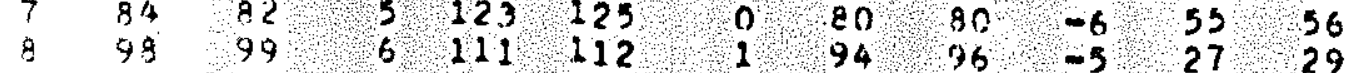

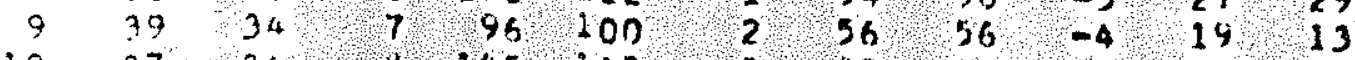

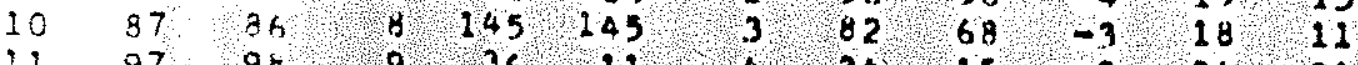

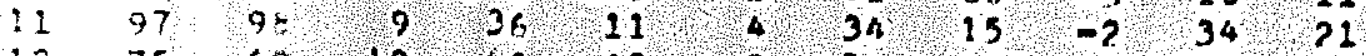

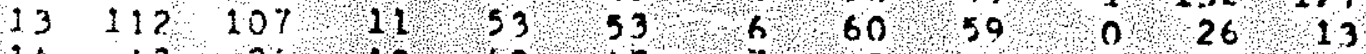

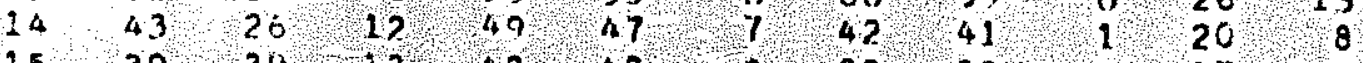

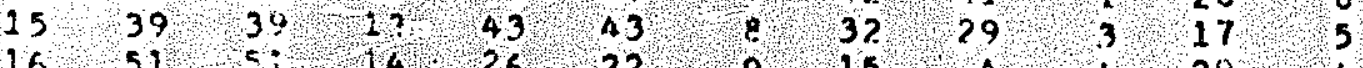

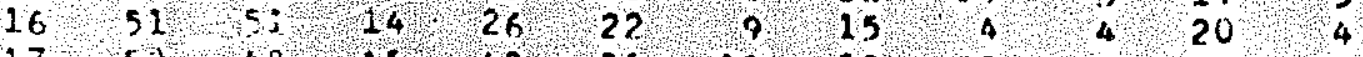

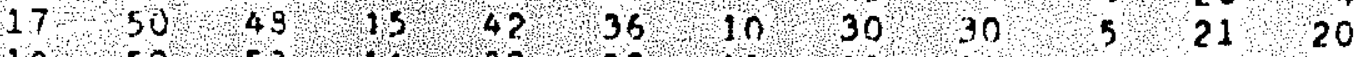

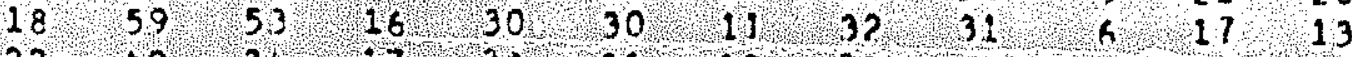

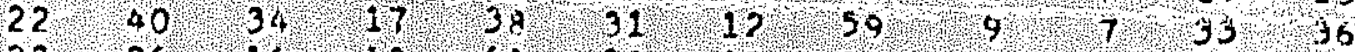

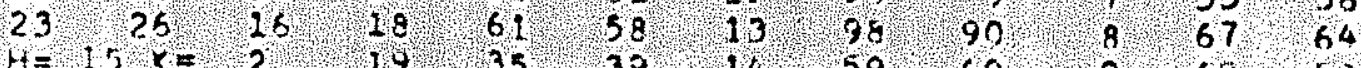

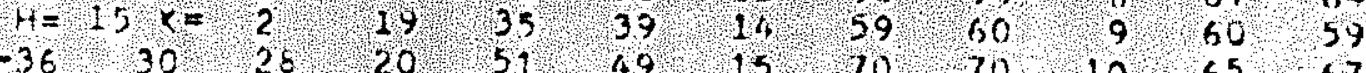

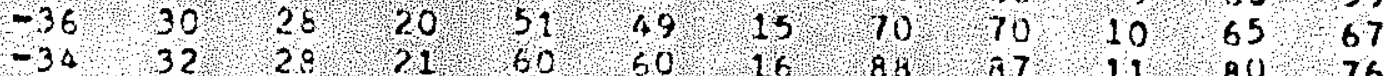

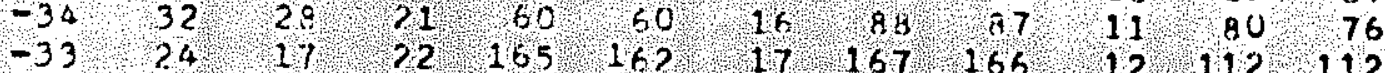

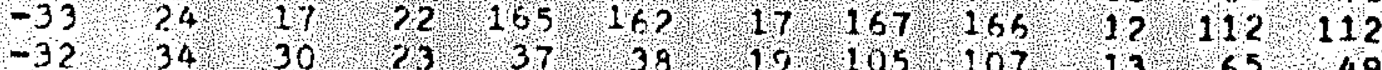




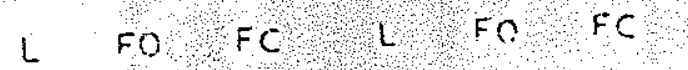

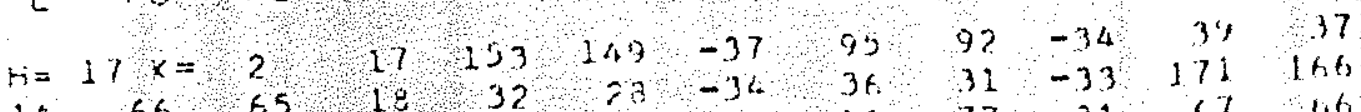

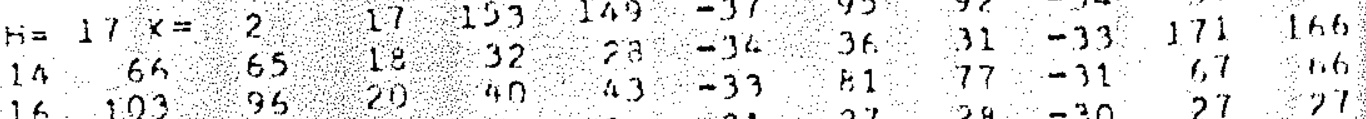

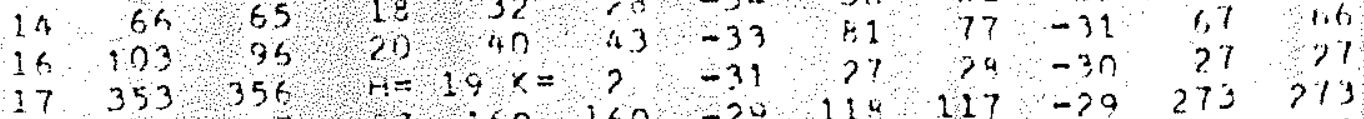

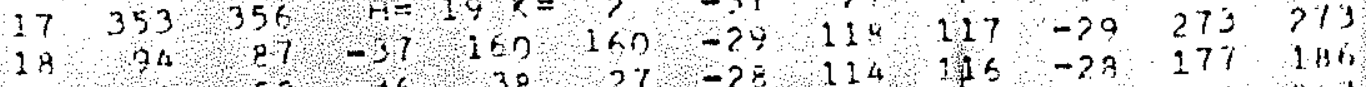

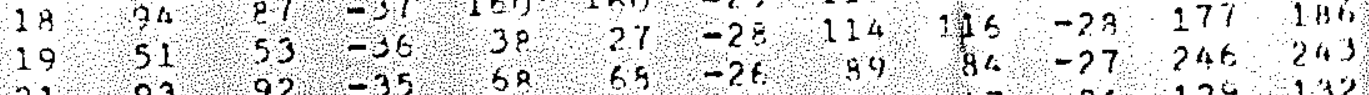

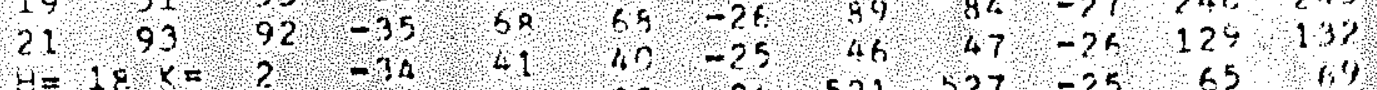

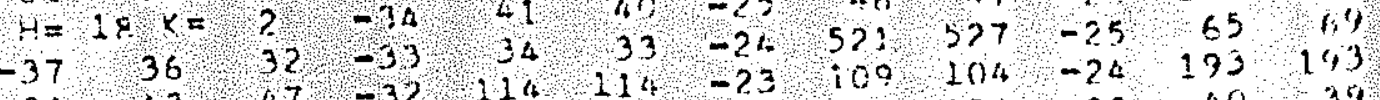

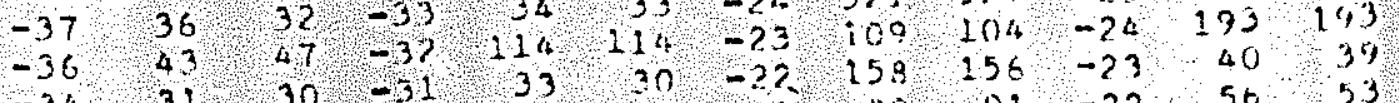

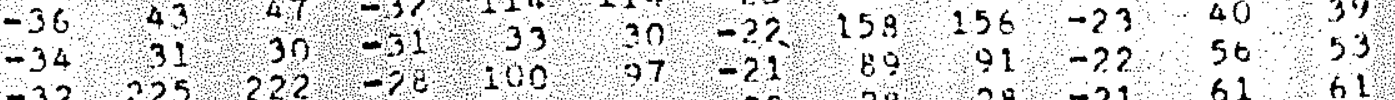

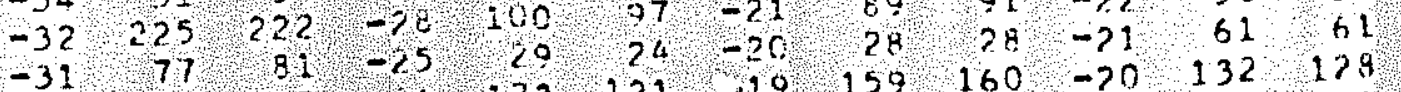

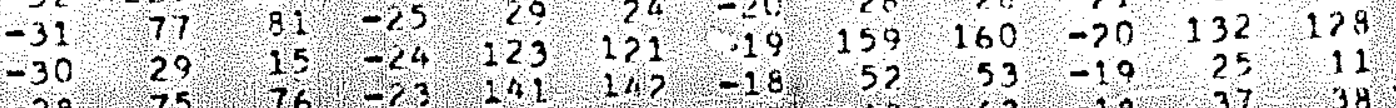

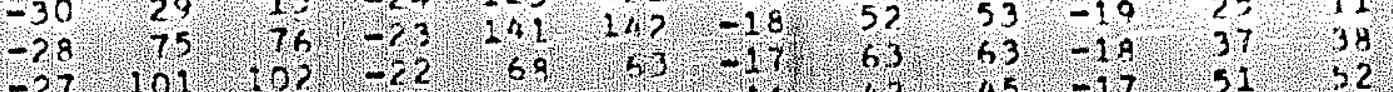

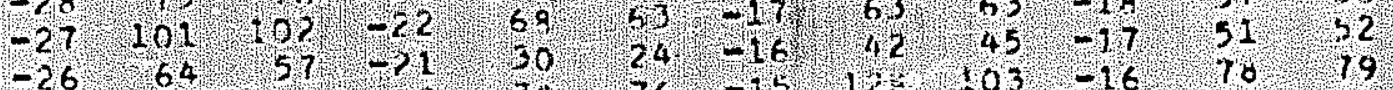

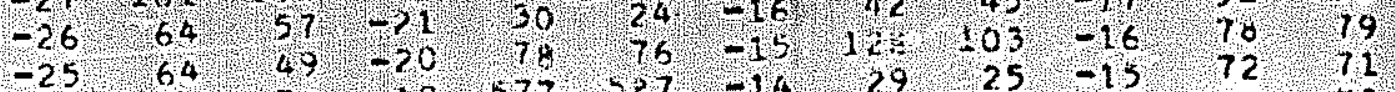

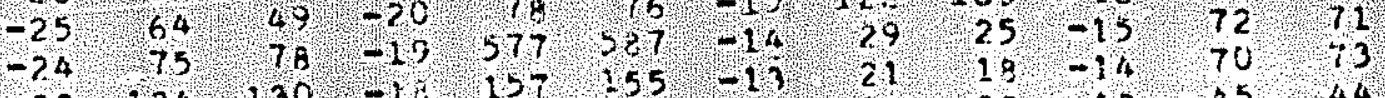

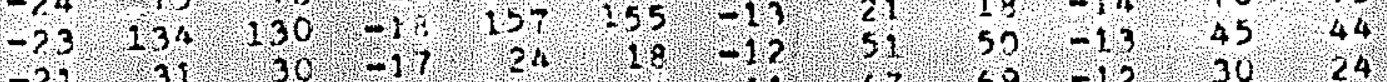

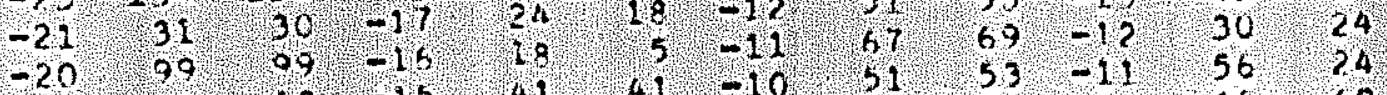

$\begin{array}{llllllllllll}-19 & 65 & 63 & -15 & 41 & 41 & -10 & 51 & 53 & -11 & 56 & 24\end{array}$

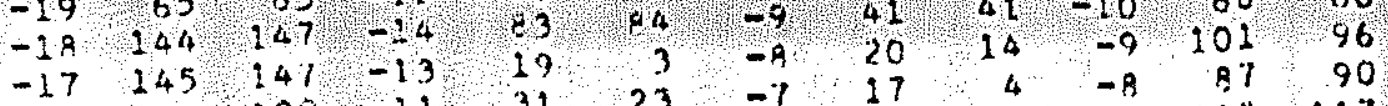

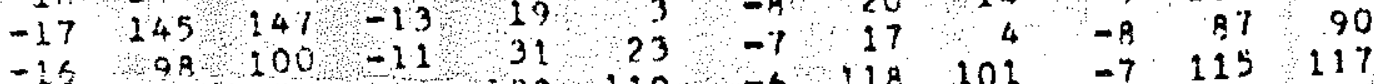

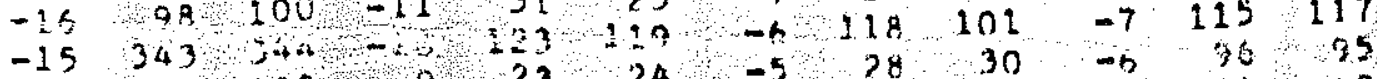

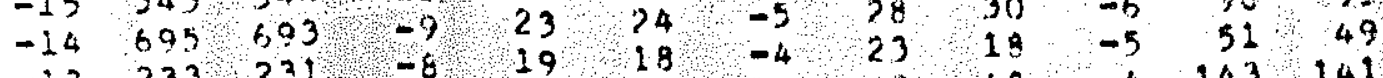

$\begin{array}{lllllllllll}-13 & 233 & 231 & -8 & 19 \\ 17 & -4 & 19 & -3 & 53 & 43 & -4 & 143 & 141\end{array}$

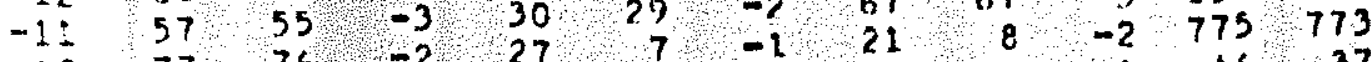

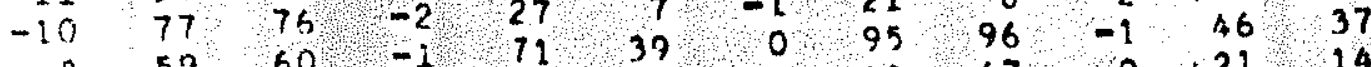

$-8 \quad 57 \quad 60 \quad-1 \quad 71,39 \quad 1 \quad 63 \quad 67 \quad 210014$

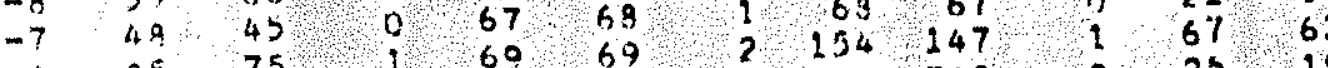

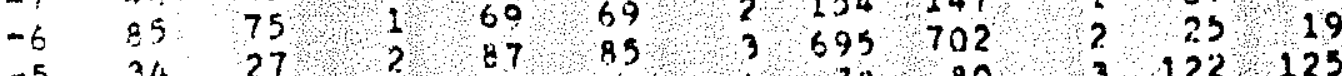

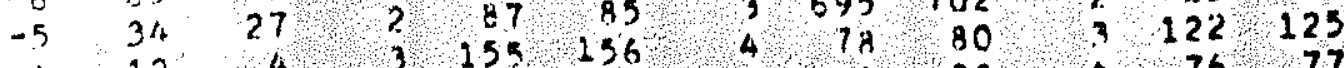

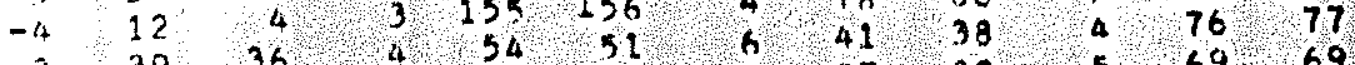

$\begin{array}{llllllllllll}-3 & 39 & 36 & 69 & 69 & 69\end{array}$

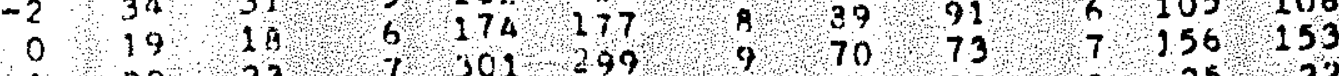

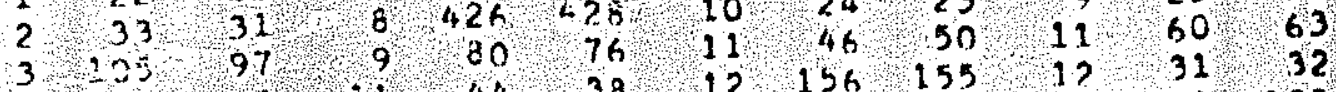

$\begin{array}{llllllllllll}4 & 72 & 5 & 1 & 1 & 44 & 39 & 12 & 156 & 155 & 12 & 31\end{array}$

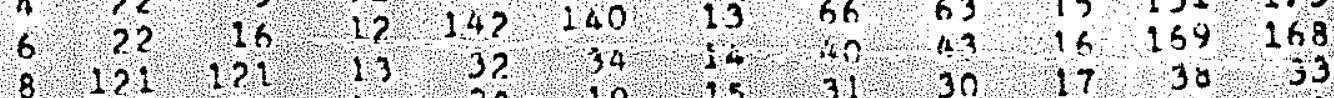

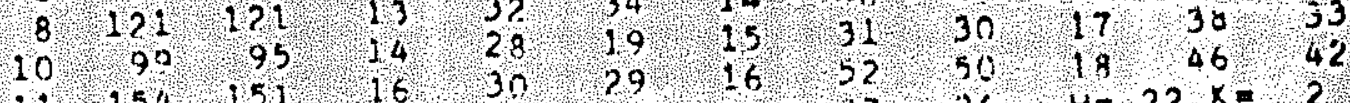

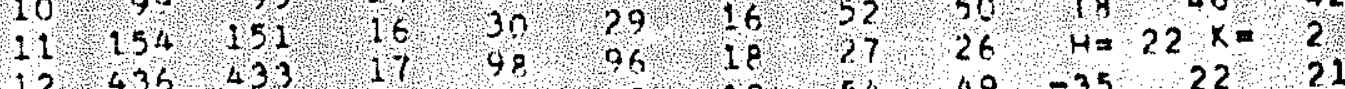

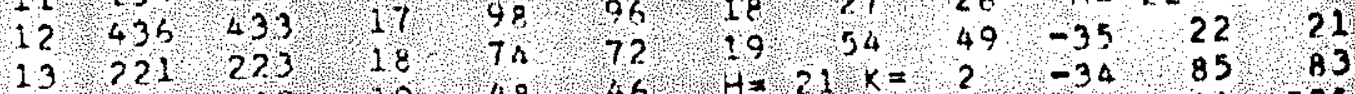

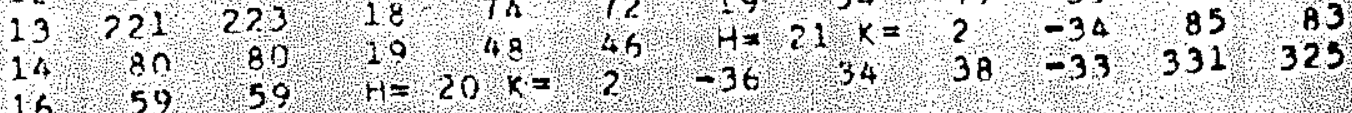

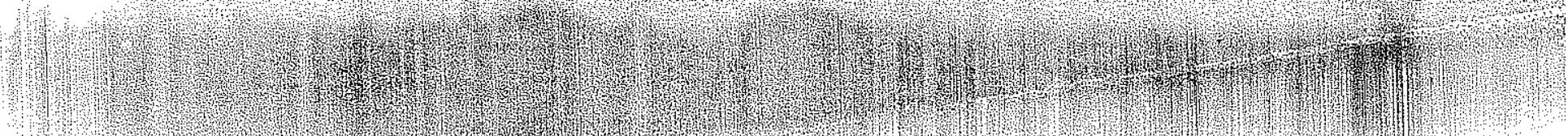




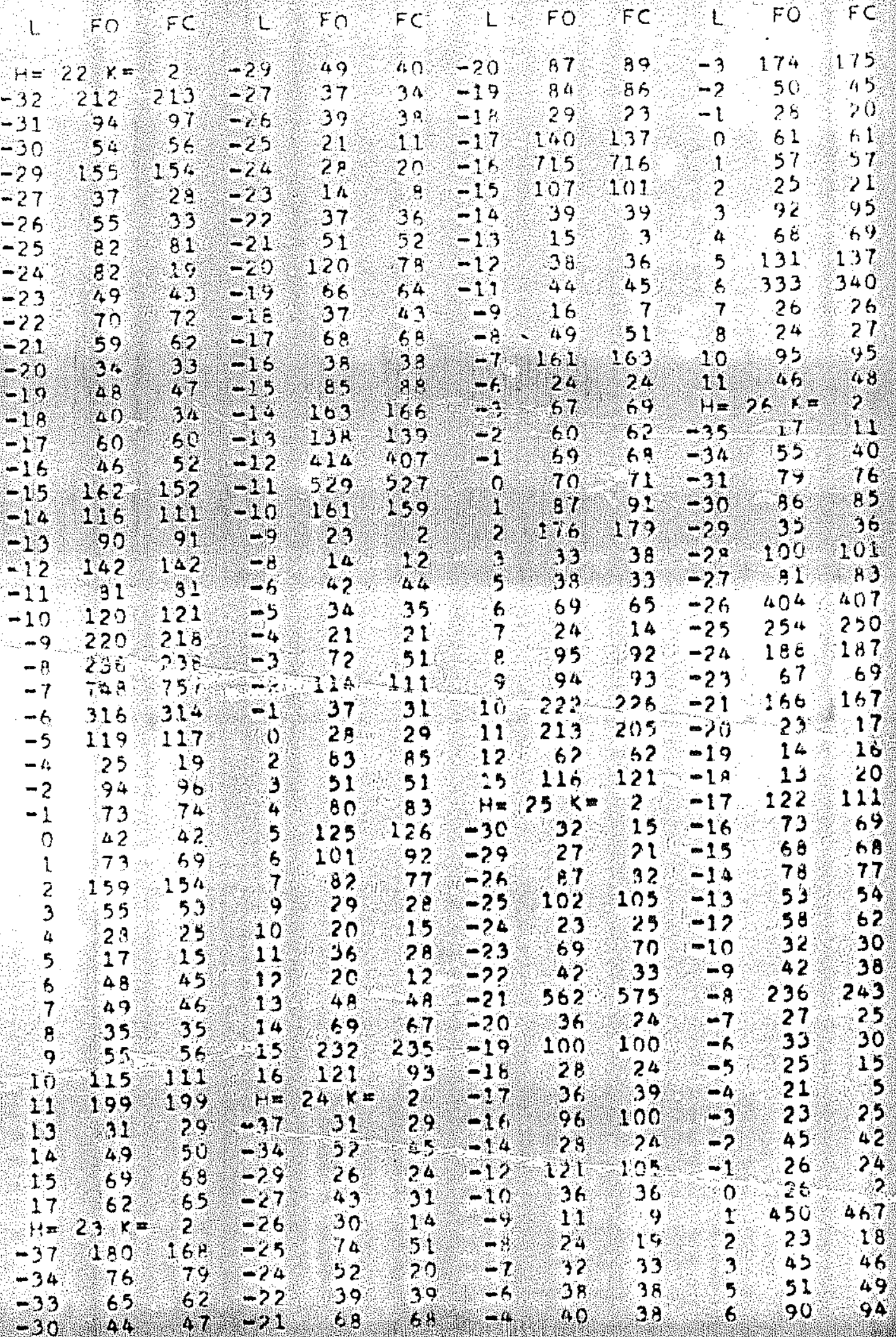


$L \quad F O=F C$

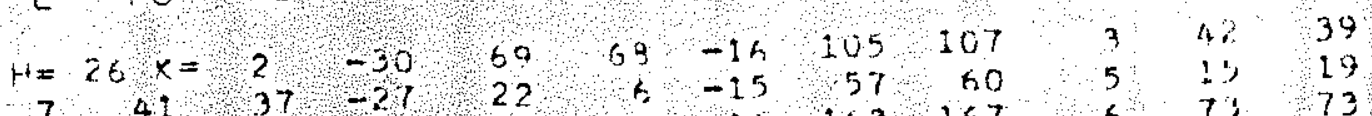

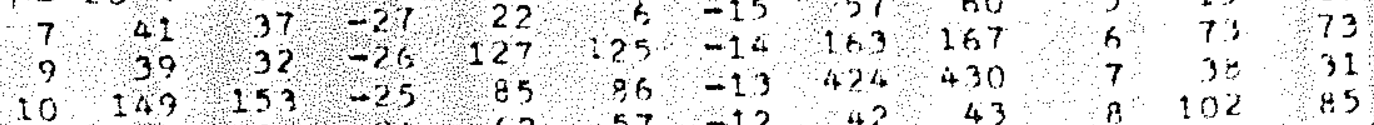

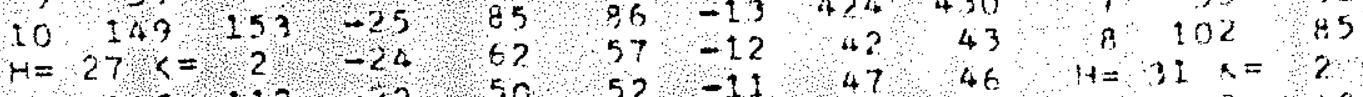

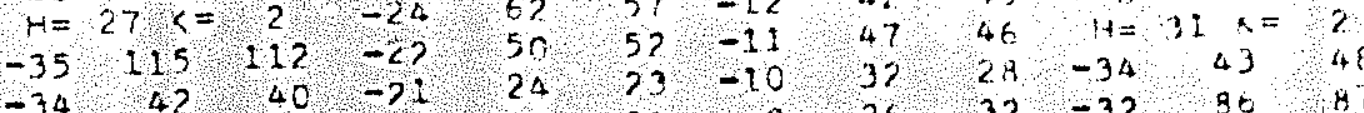

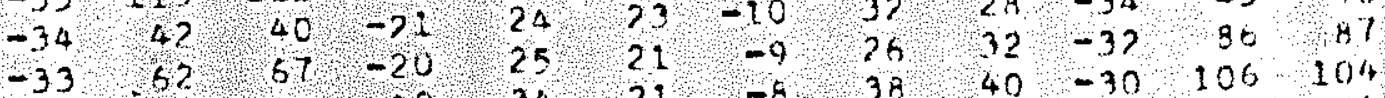

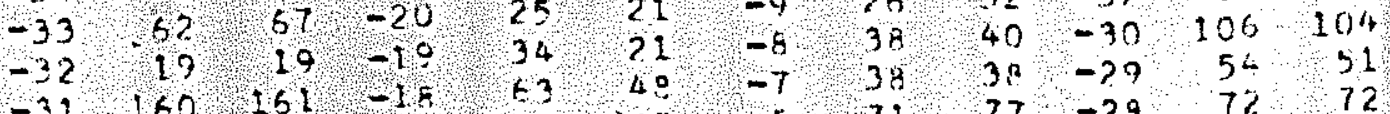

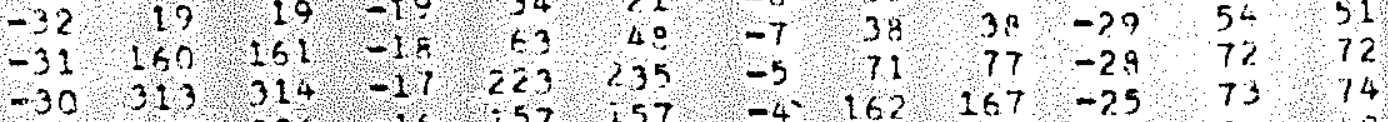

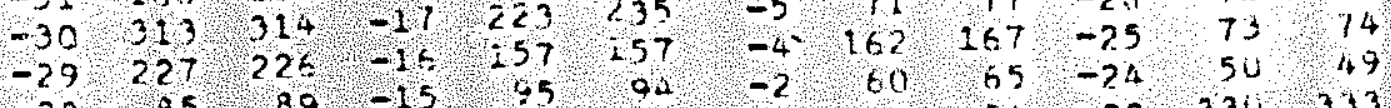

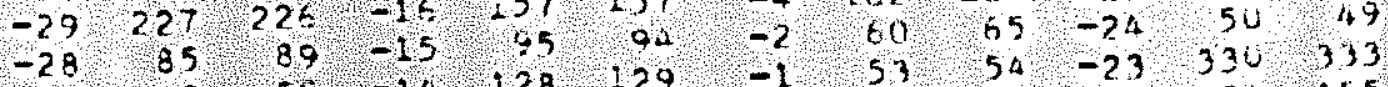

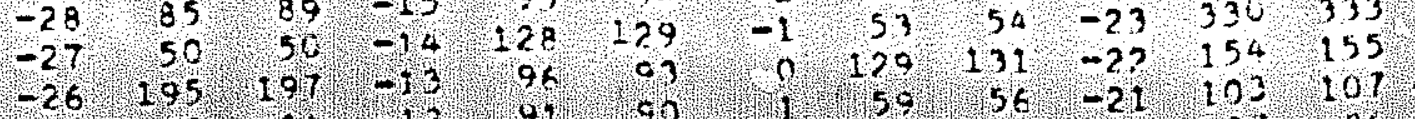

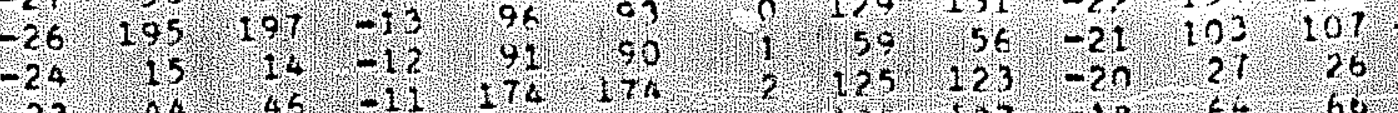

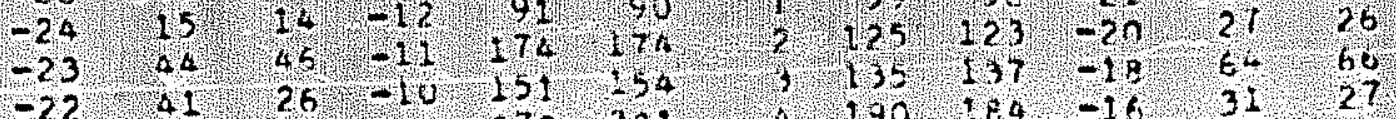

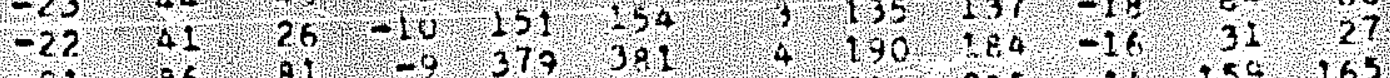

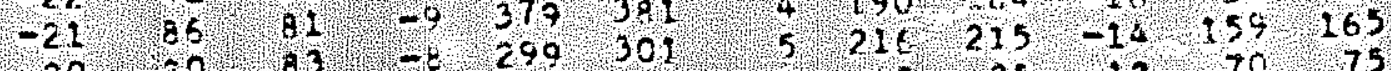

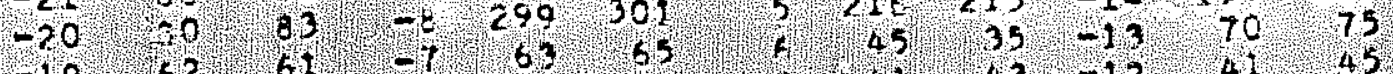

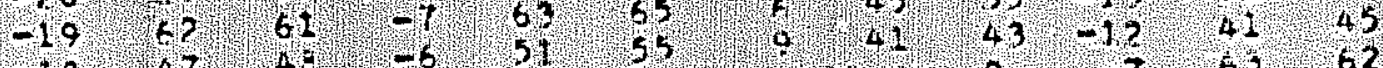

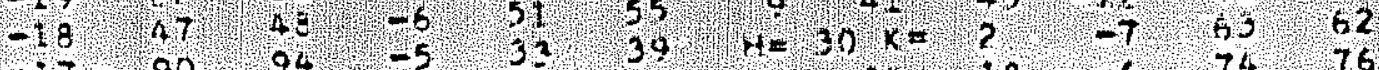

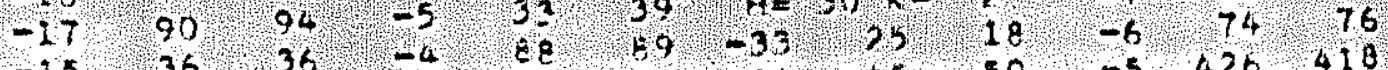

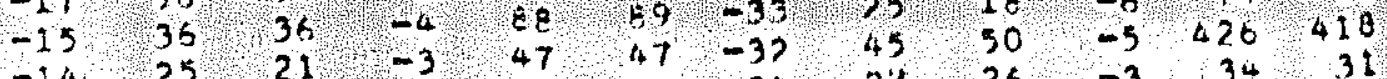

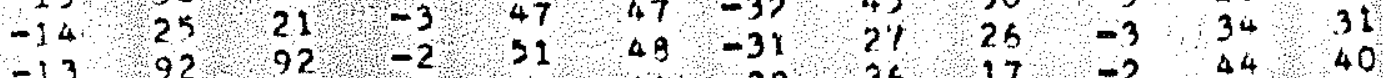

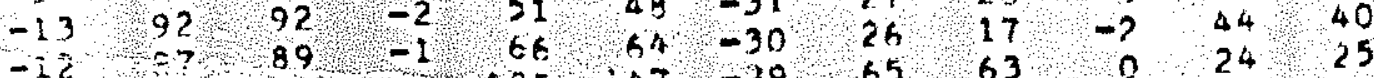

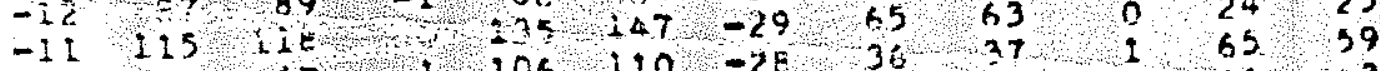

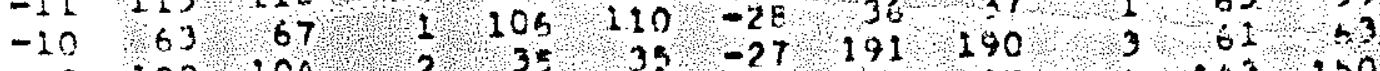

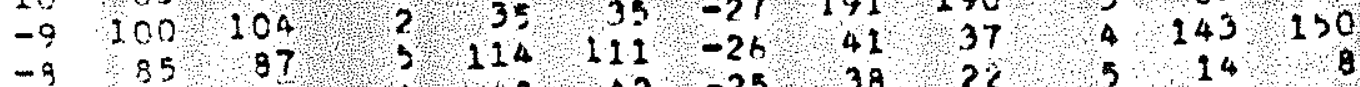

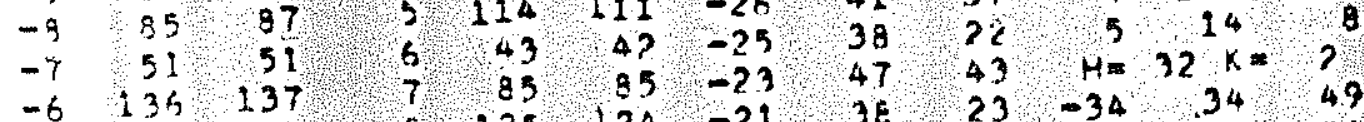

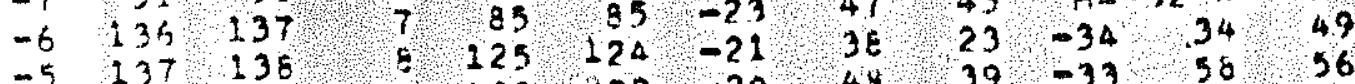

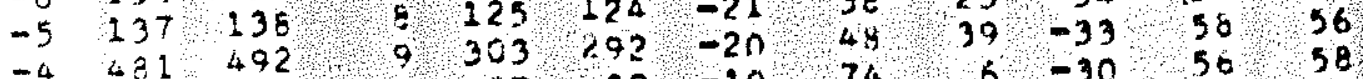

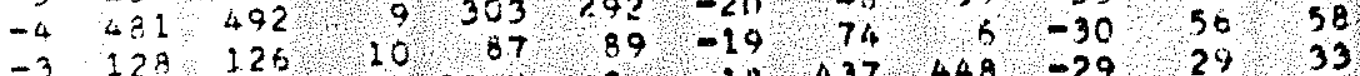

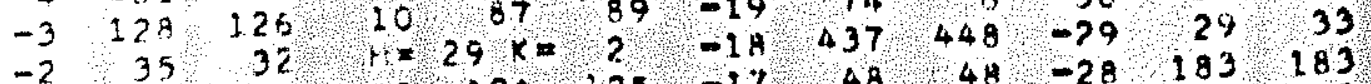

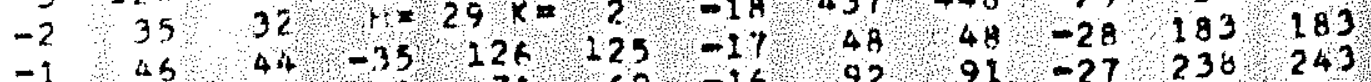

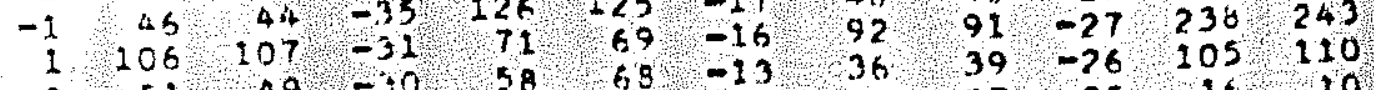

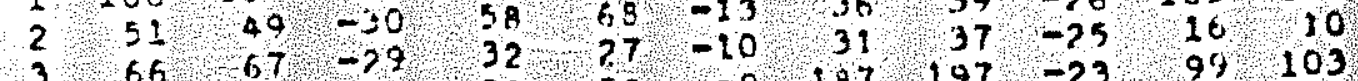

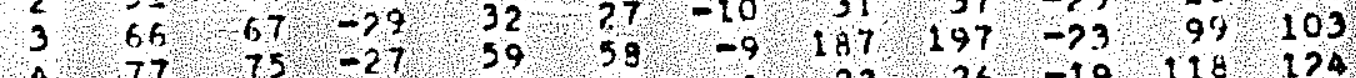

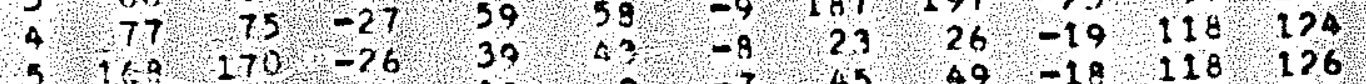

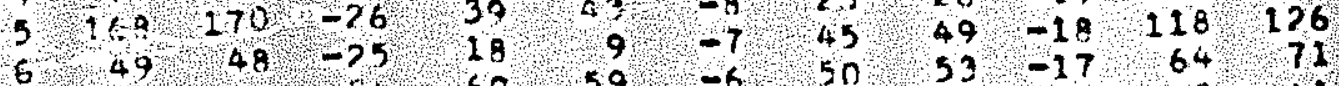

$\begin{array}{lllllllllllll}7 & 44 & 44 & -24 & 60 & 59 & -6 & 50 & 53 & -17 & 64 & 71 \\ 9 & 50 & 62 & -2 & 27 & 32 & -5 & 58 & 92 & -16 & 43 & 41\end{array}$

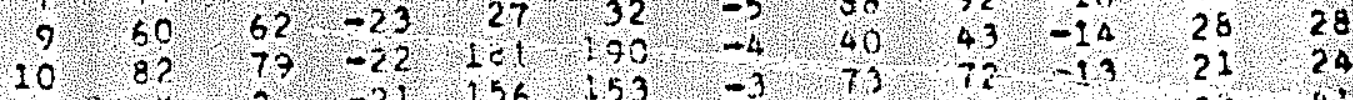

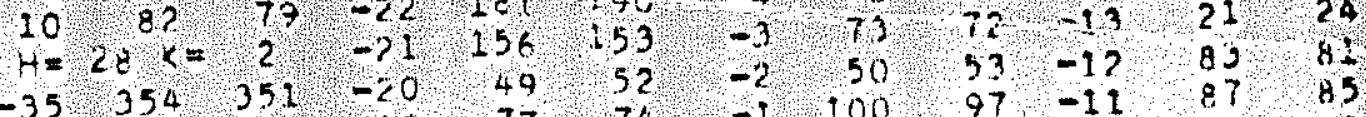

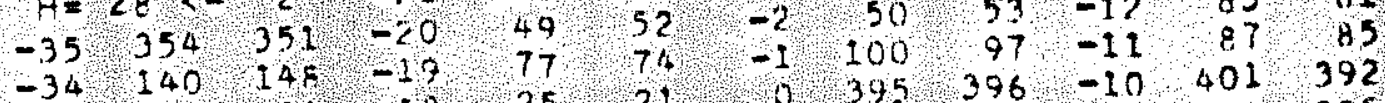

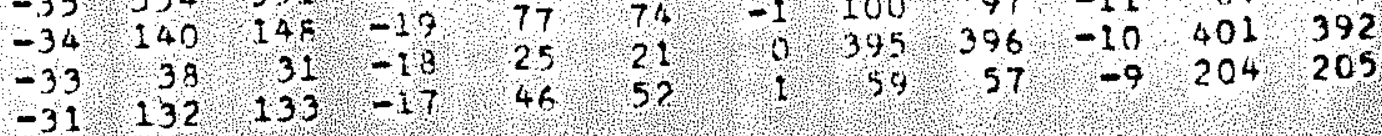




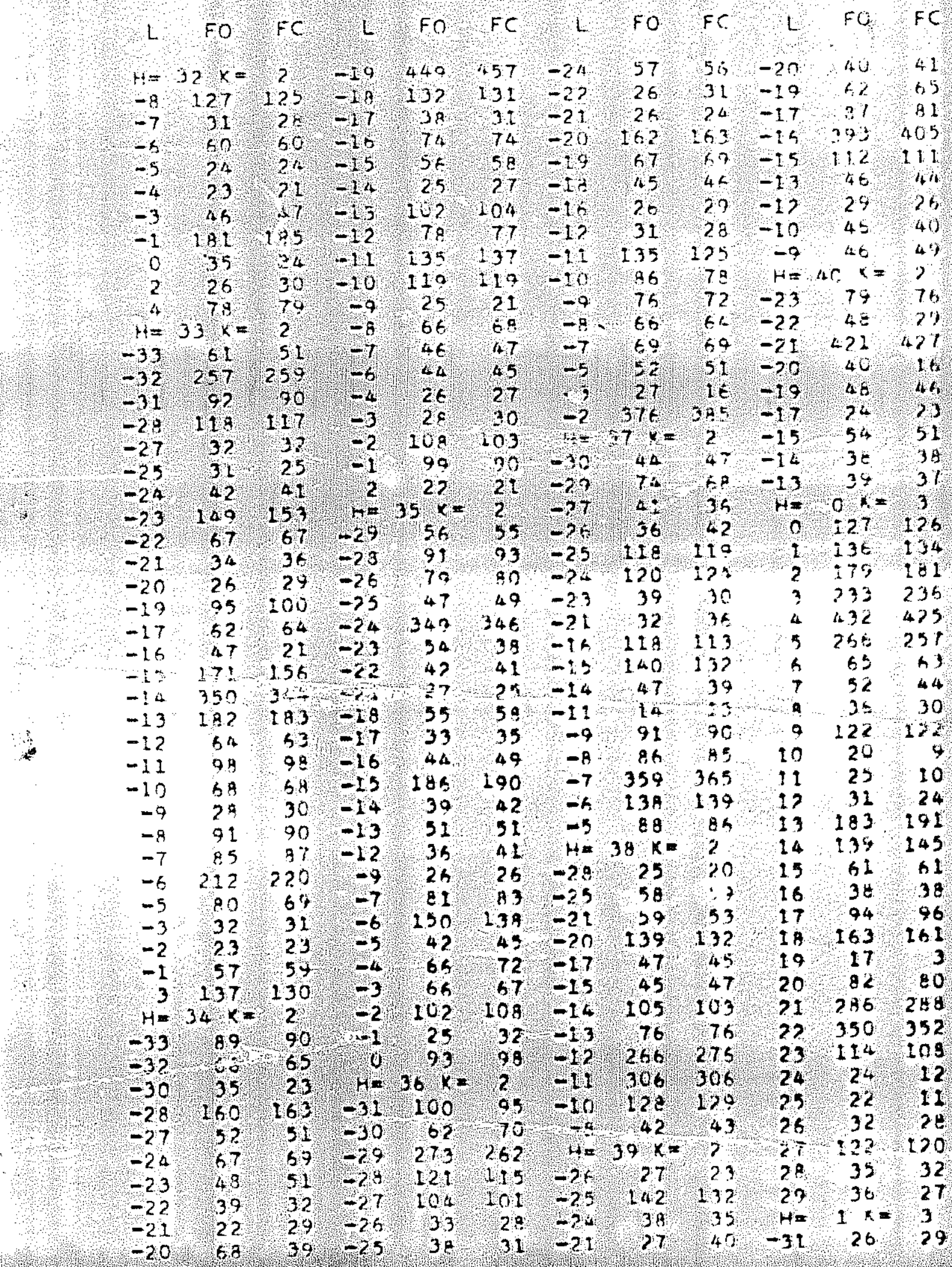




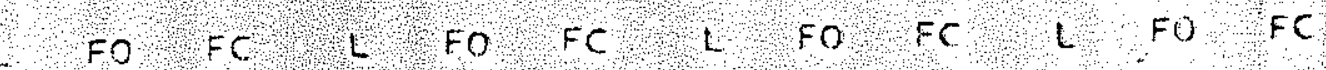

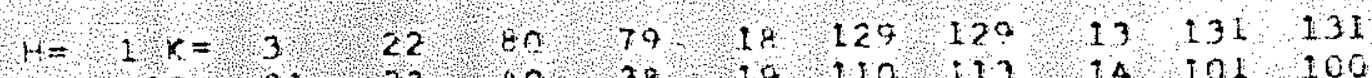

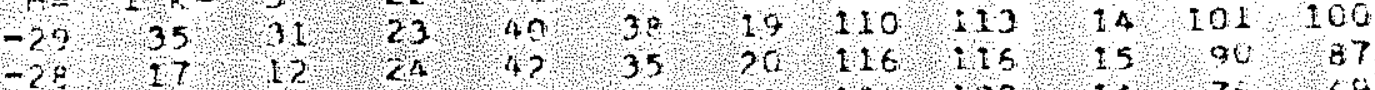

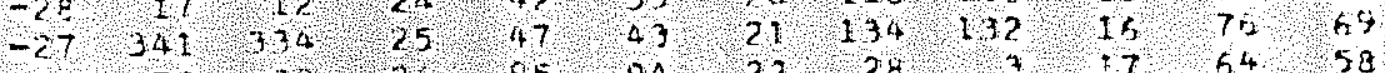

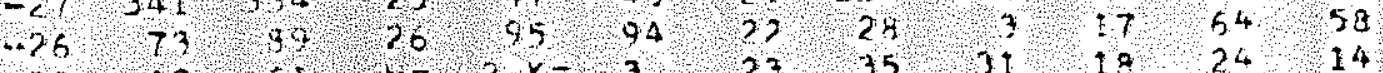

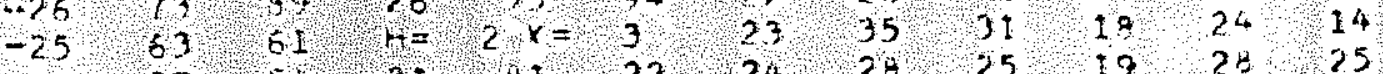

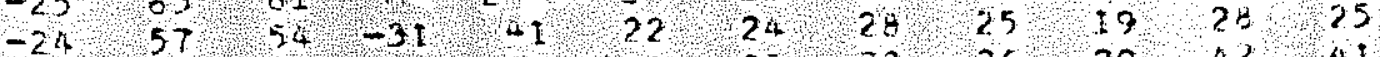

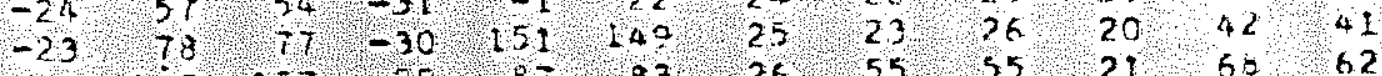

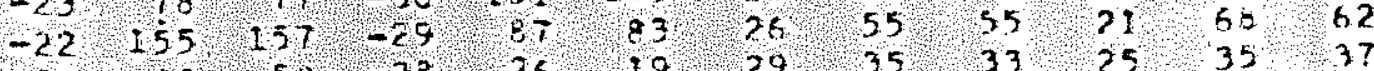

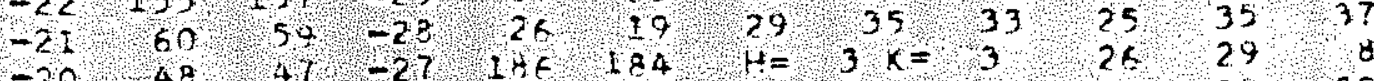

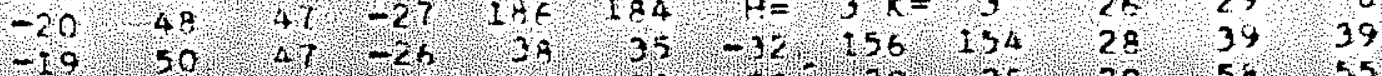

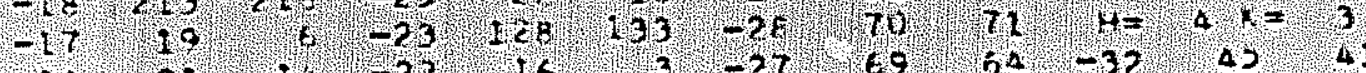

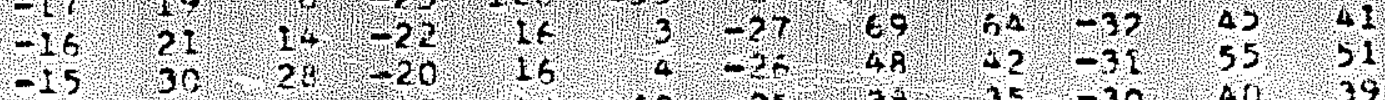

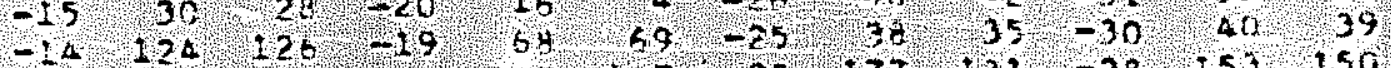

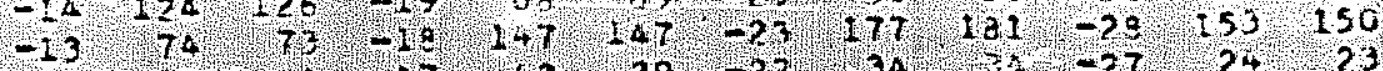

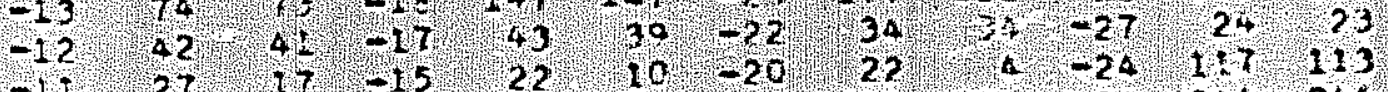

$\begin{array}{lllllllllllllll}-12 & 27 & 17 & -15 & 22 & 10 & -20 & 28 & 256 & -23 & 744 & 246\end{array}$

$610609-13 \quad 13$ 12 -1 * $247 \quad 245 \quad-22 \quad 2042014$

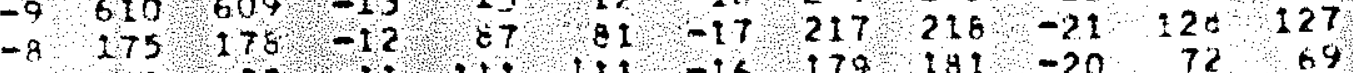

$-7991 \times 93 \times-110111411,-16$ 179 131 $-20 \quad 72 \quad 69$

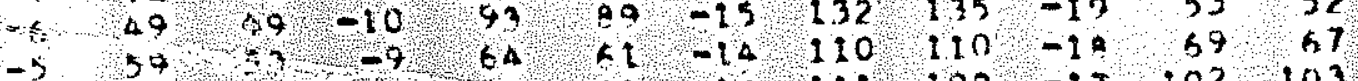

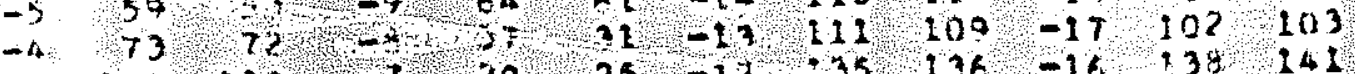

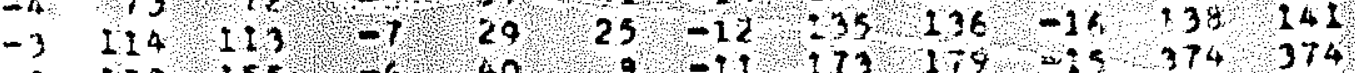

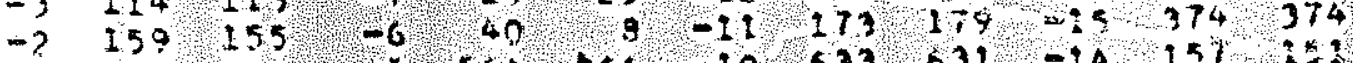

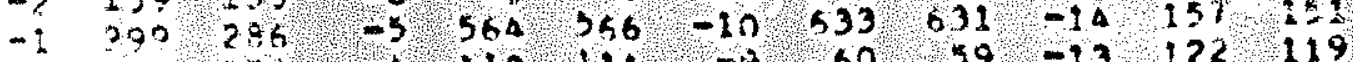

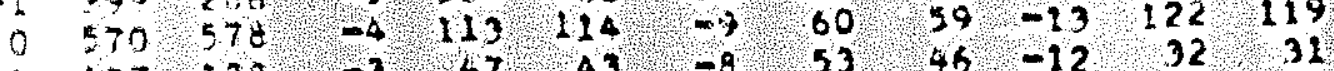

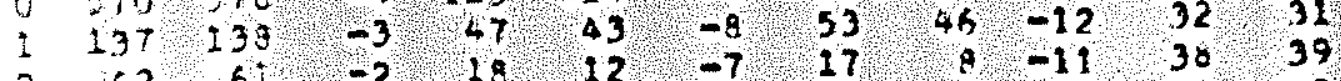

$\begin{array}{lllllllllll}2 & 62 & 61 & -2 & 19 & 12 & -7 & 17 & 9 & -11 & 30\end{array}$

$\begin{array}{lllllllllll}3 & 18 & 9 & -1 & 28 & 10 & -6 & 53 & 99 & -10 & 6\end{array}$

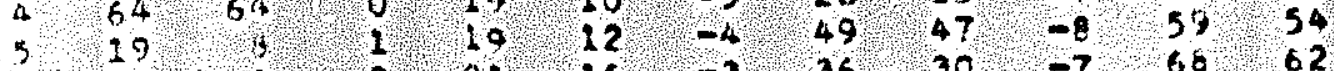

$\begin{array}{lllllllllll}6 & 12 & 3 & 2 & 21 & 15 & -3 & 36 & 30 & -7 & 66 \\ 90 & 96 & -2 & 37 & 16 & -6 & 316 & 327\end{array}$

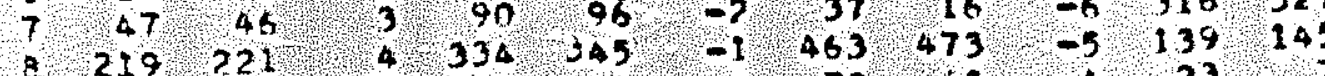

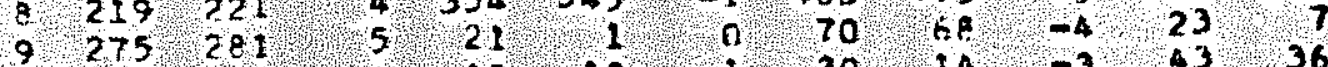

$\begin{array}{llllllllllllll}10 & 54 & 50 & 6 & 19 & 18 & 1 & 30 & 14 & -3 & 43 & 36\end{array}$

$\begin{array}{lllllllllllllll}11 & 26 & 12 & 7 & 16 & 2 & 2 & 17 & 3 & -2 & 35 & 40\end{array}$

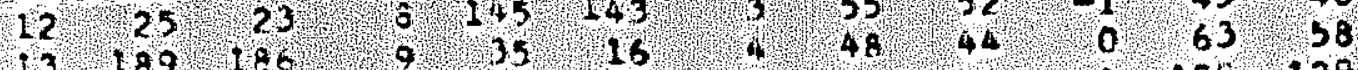

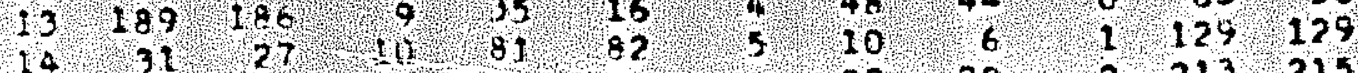

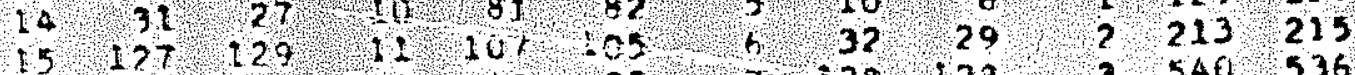

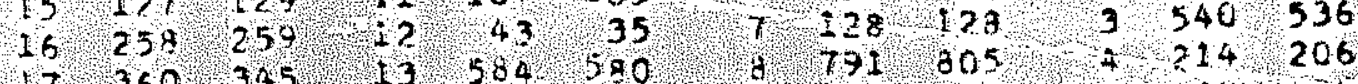

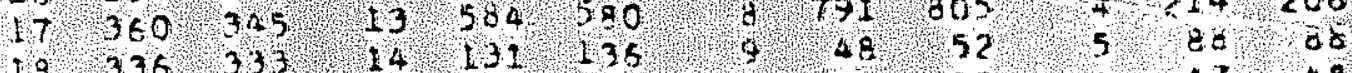

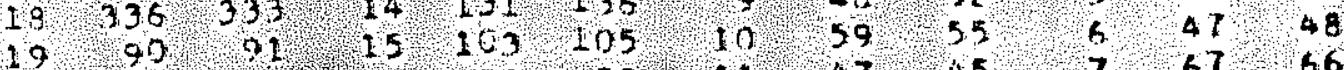

$\begin{array}{lllllllllll}20 & 72 \\ 3\end{array}$ 


\begin{tabular}{|c|c|c|c|c|c|c|c|c|c|c|c|}
\hline$H=$ & $6 \mathrm{~K}=$ & 3 & 3 & 24 & 19 & -4 & 105 & 105 & -8 & 21 & 8 \\
\hline 9 & 14 & 88 & 4 & 54 & 51 & -3 & 931 & 79 & -7 & 36 & 35 \\
\hline 10 & 25 & 18 & 5 & 87 & 89 & $-?$ & $\mathrm{KS}$ & 60 & -5 & 23 & 18 \\
\hline 11 & 46 & 1.9 & 6 & 108 & 107 & -1 & 52 & 52 & -5 & 37 & 31 \\
\hline 12 & 140 & 141 & 7 & 154 & 150 & 0 & 41 & 43 & -4 & 19 & 8 \\
\hline 14 & 15 & 3 & 5 & 33 & 222 & 1 & Es & e3 & -3 & 63 & 4 \\
\hline 25 & 24 & 22 & 19 & 17 & 21 & 2 & 101 & 59 & -2 & 157 & 149 \\
\hline 1 & -37 & 33 & 10 & 10 & 4 & 3 & 091 & 77 & -1 & 37 & 34 \\
\hline 17 & 25 & 21 & 11 & 13 & 13 & 4 & 13 & 10 & $n$ & 16 & 7 \\
\hline 20 & 73 & 78 & 12 & 61 & 80 & 5 & 18 & 23 & 1 & 28 & 23 \\
\hline 2 & 30 & 10 & 14 & 14 & 10 & 6 & 29 & 29 & 2 & 16 & 18 \\
\hline & 50 & 53 & 15 & 52 & 4.5 & 7 & 59 & 56 & 3 & 21 & 16 \\
\hline 23 & 65 & 65 & 16 & 34 & 41 & 8 & 16 & 15 & 4 & 19 & 6 \\
\hline 24 & 71 & 71 & 17 & 42 & 46 & 9 & 25 & 20 & 5 & 17 & 15 \\
\hline 25 & 161 & 157 & 12 & 39 & 32 & 10 & 10 & 3 & 6 & 108 & 106 \\
\hline 26 & 28 & 26 & 19 & 39 & 36 & 11 & 116 & 123 & 7 & 69 & 73 \\
\hline 21 & 95 & $\mathrm{ag}$ & 20 & 91 & 88 & 12 & 25 & 29 & 9 & 42 & 43 \\
\hline 28 & 63 & oo & $2 !$ & 106 & 104 & 13 & 91 & 25 & 29 & 44 & 43 \\
\hline & $5 x=$ & 3 & 22 & 37 & 25 & 16 & 30 & 32 & 10 & 48 & 46 \\
\hline-3 & 35 & 60 & 23 & 40 & 2 & 16 & $1 \%$ & 290 & 11 & 192 & 192 \\
\hline-31 & 38 & 35 & 21 & 63 & 83 & 17 & 31 & 27 & 17 & 42 & 39 \\
\hline-2 & 46 & 40 & 25 & 364 & 352 & 18 & 133 & 131 & 13 & 162 & 162 \\
\hline-28 & 232 & 227 & 26 & 58 & 53 & 14 & 241 & 244 & 14 & 225 & 277 \\
\hline-27 & 74 & 6,6 & 28 & 30 & 29 & 20 & 343 & 353 & 15 & 1332 & 331 \\
\hline if & 29 & 13 & $r=$ & $\theta<=$ & 24 & 21 & 211 & $20 ?$ & 13 & 460 & 466 \\
\hline & 16 & 20 & -33 & 147 & 144 & 27 & 45 & 45 & 17 & 80 & 88 \\
\hline & 2 & 5 & -32 & 25 & 12 & 23 & 19 & 23 & 19 & 46 & 43 \\
\hline & 28 & 2 & 11 & के & 65 & 24 & 45 & 46 & 10 & 20 & 26 \\
\hline-21 & 33 & 29 & -30 & 73 & 69 & 23 & 160 & $2 \leq 3$ & 20 & 162 & 163 \\
\hline- & 123 & 117 & -29 & 58 & 31 & HE & $7 \mathrm{k}=$ & 3 & 21 & 43 & 38 \\
\hline $\begin{array}{l}-10 \\
-: 8\end{array}$ & 385 & 382 & -28 & 36 & 29 & -33 & 37 & 34 & $2 ?$ & 28 & 26 \\
\hline-13 & 157 & 157 & -27 & 26 & 25 & -32 & 43 & 38 & 24 & 17 & 13 \\
\hline $\begin{array}{l}-17 \\
-16\end{array}$ & 37 & 34 & -76 & 39 & 39 & -31 & 54 & 51 & 2.5 & 66 & 61 \\
\hline-16 & 24 & 9 & -25 & 27 & 21 & -30 & 50 & 50 & 26 & 37 & 37 \\
\hline 75 & 103 & 105 & -24 & 330 & 336 & -29 & 232 & 229 & $H=$ & $8 \mathrm{x}=$ & 3 \\
\hline & 72 & 73 & -31 & 145 & 242 & $-2 A$ & 50 & 37 & -33 & 86 & 92 \\
\hline & 42 & 39 & -20 & 103 & 103 & -27 & 86 & 86 & -32 & 149 & 141 \\
\hline 12 & 65 & 52 & -19 & 101 & 100 & -26 & 40 & 38 & -31 & 30 & 58 \\
\hline$;$ & 224 & 227 & -19 & 223 & 18 & -23 & 45 & 43 & -29 & 145 & 144 \\
\hline-1 & 259 & 265 & -17 & 25 & 24 & -24 & 131 & 132 & -26 & 25 & 73 \\
\hline- & 25 & 16 & -16 & 30 & 91 & -20 & 225 & 228 & -25 & 132 & 138 \\
\hline- & 24 & 18 & -15 & 207 & 212 & -19 & 30 & 13 & -21 & B2 & 83 \\
\hline$=$ & 59 & $4 c$ & -14 & 13 & 2 & $-1 a$ & 22 & 10 & -20 & 174 & 172 \\
\hline-8 & 23 & 14 & -13 & 16 & 4 & -17 & 19 & 13 & -19 & 26 & 18 \\
\hline- & 301 & 103 & $41)$ & 16 & e & -16 & 125 & 124 & -18 & 76 & 77 \\
\hline-2 & 153 & 158 & -11 & 109 & 107 & -15 & 115 & 114 & -17 & 77 & 79 \\
\hline- & 208 & 212 & -10 & 107 & 200 & $-1 \%$ & 24 & 17 & -16 & 538 & 533 \\
\hline-2 & 563 & $55 \%$ & 1 -9 & 193 & 95 & -13 & 43 & 38 & -14 & 151 & 148 \\
\hline-1 & 530 & 530 & -8 & $2 ?$ & 83 & $-1 ?$ & 20 & 47 & -13 & 114 & 116 \\
\hline & 219 & 212 & -7 & 237 & 223 & -11 & $75 \%$ & $77 ?$ & -12 & 63 & 63 \\
\hline & 117 & 110 & -6 & 520 & 220 & -10 & 122 & 127 & -11 & 153 & 150 \\
\hline 2 & 90 & 71 & - & $2+1$ & 213 & $>-9$ & 19 & 1414 & -10 & 56 & 54 \\
\hline
\end{tabular}




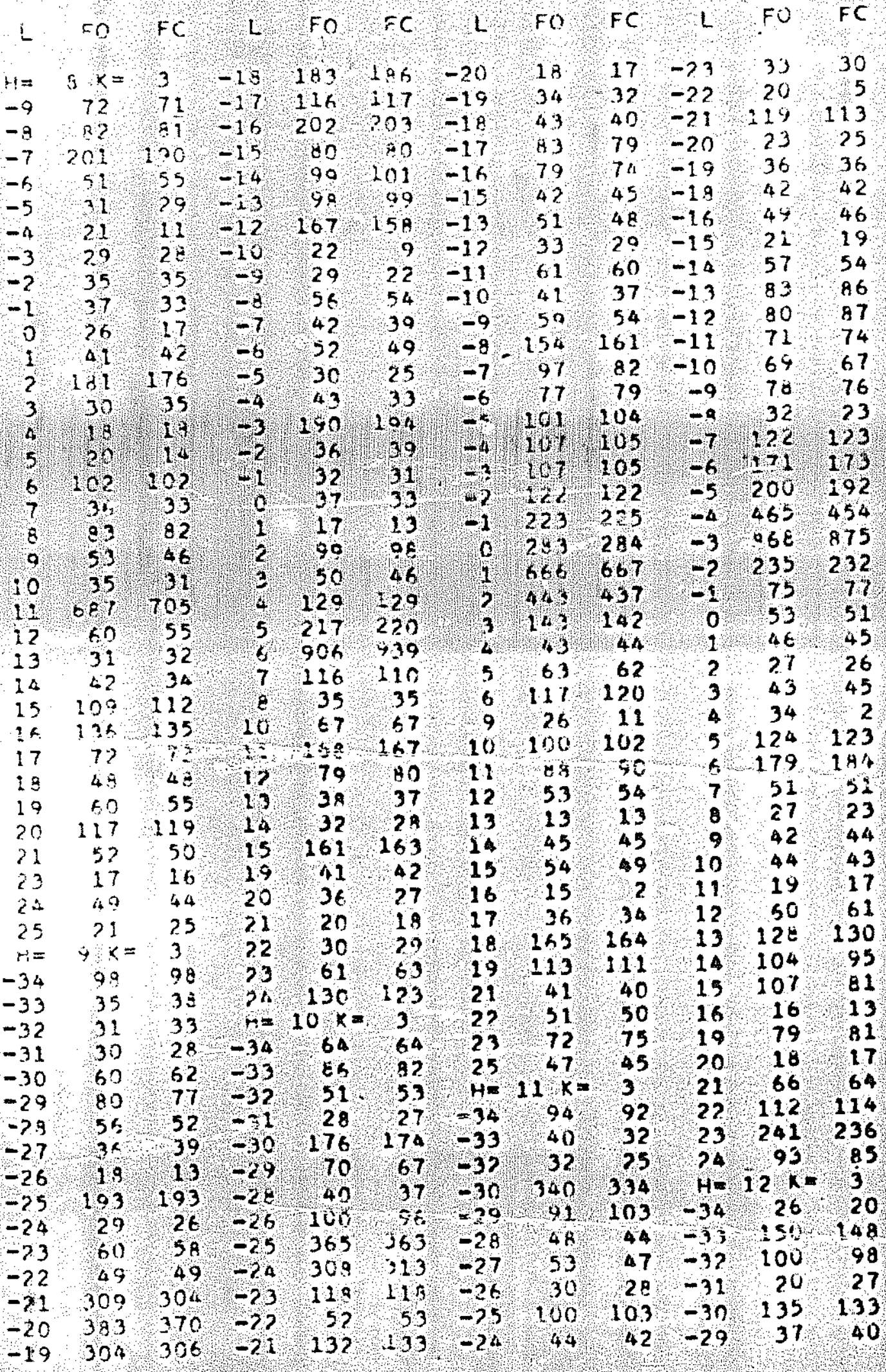




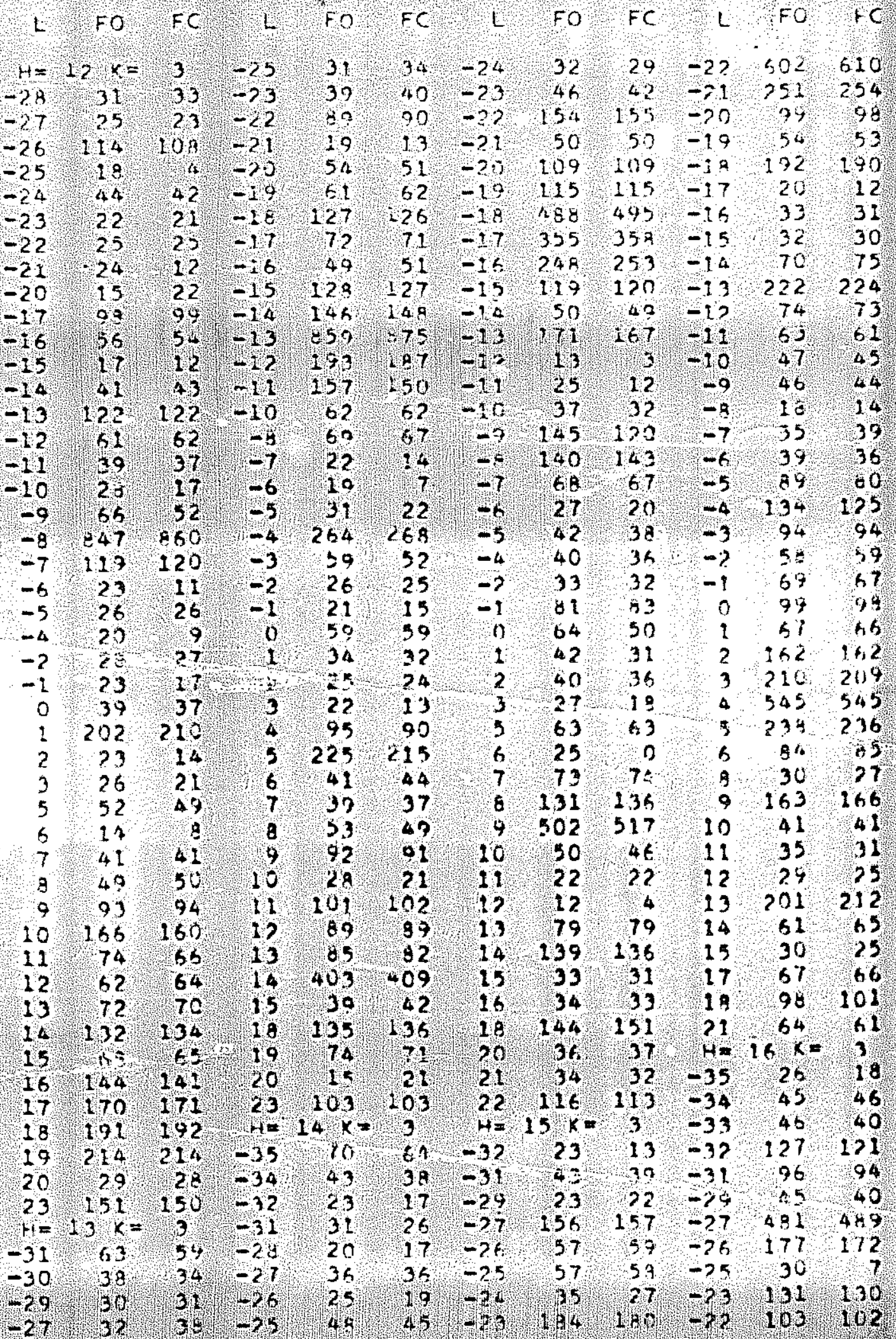




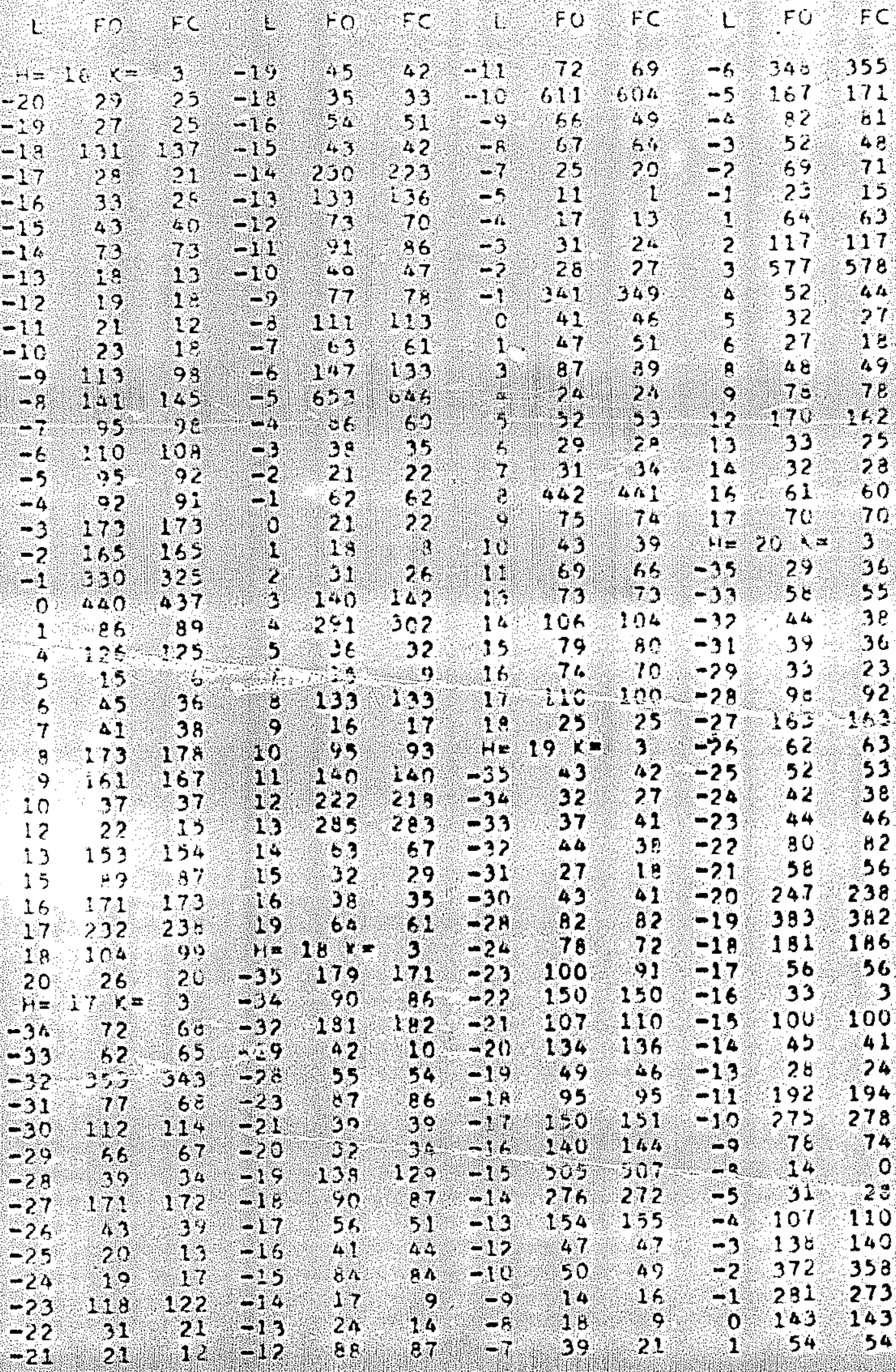




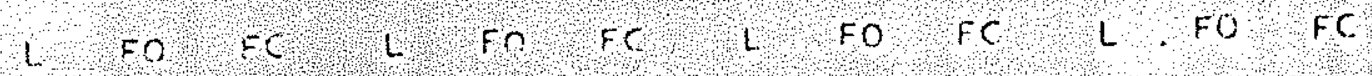

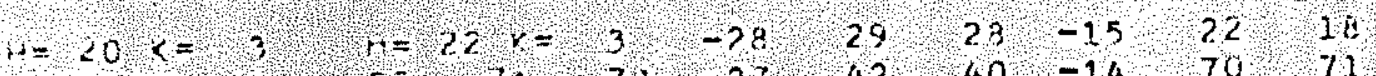

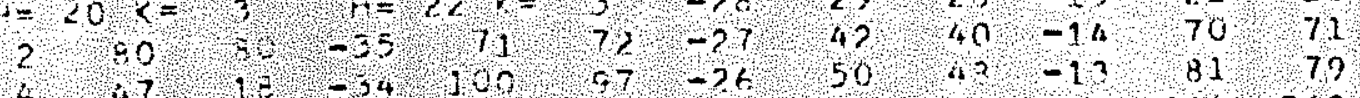

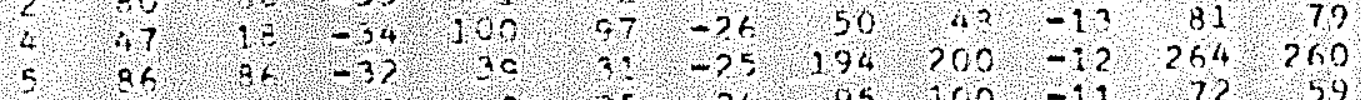

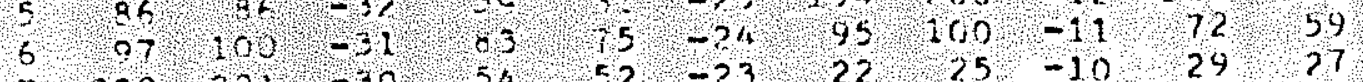

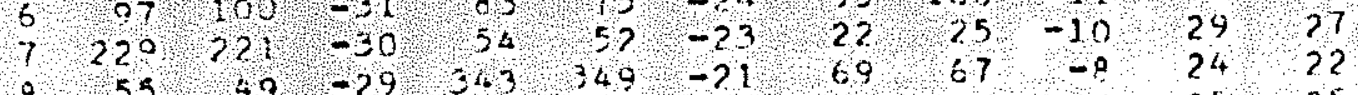

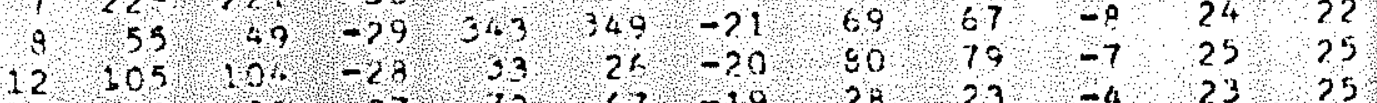

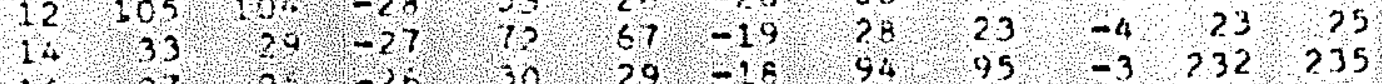

$\begin{array}{lllllllllllll}16 & 97 & 9 & -36 & 30 & 29 & -16 & 94 & 95 & -3 & 232 & 23\end{array}$

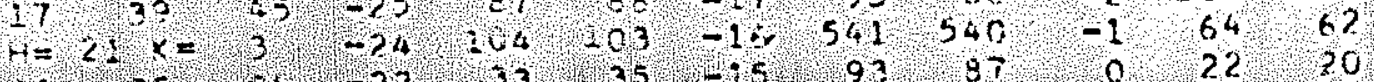

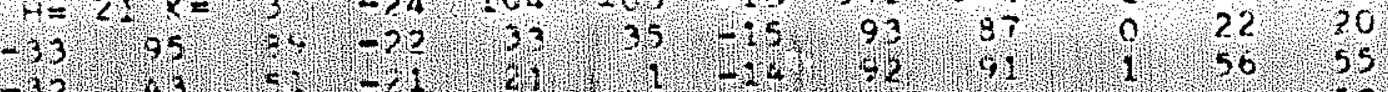

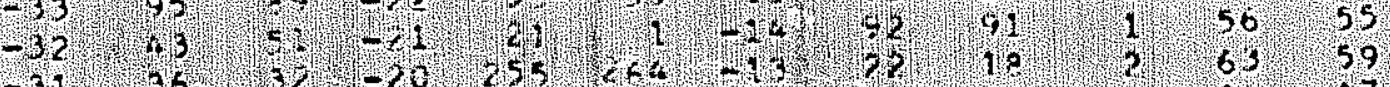

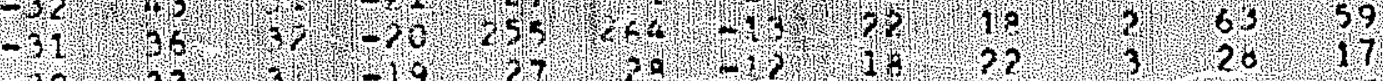

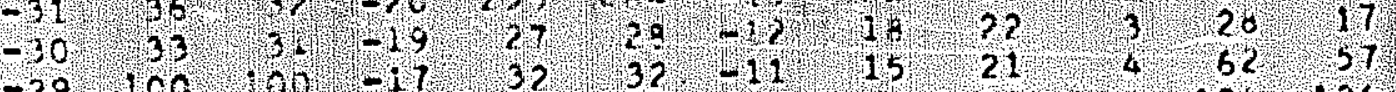

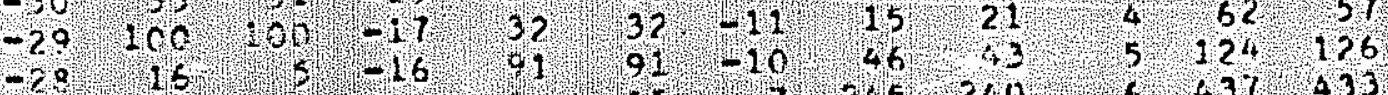

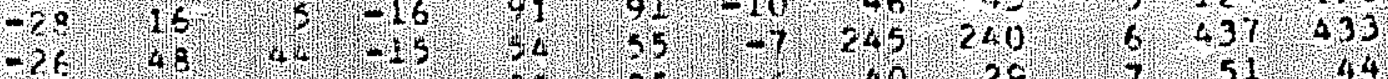

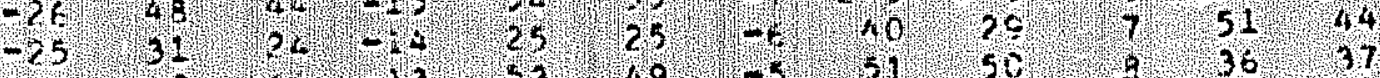

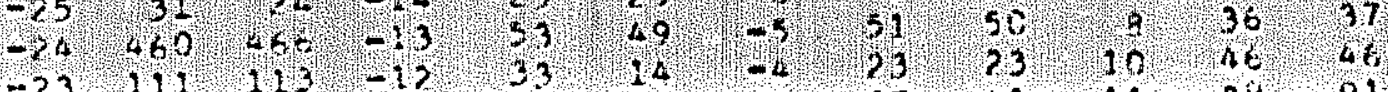

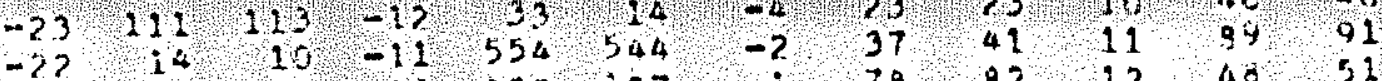

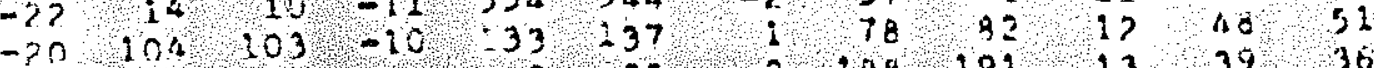

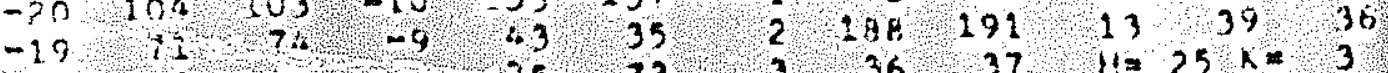

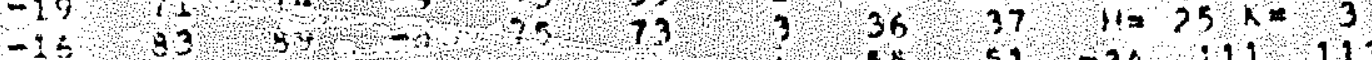

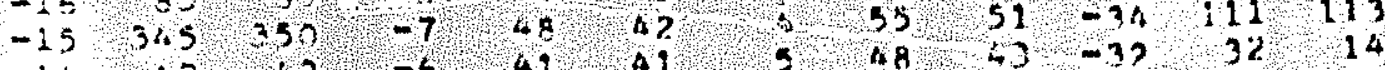

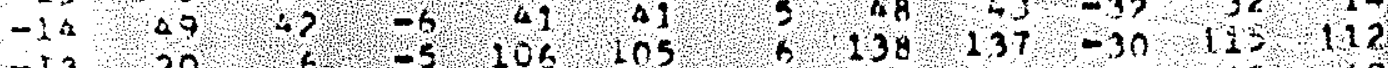

$\begin{array}{llllllllllll}-13 & 20 & 6 & -5 & 109 & 75 & 6 & 77 & 75 & -29 & 60 & 6\end{array}$

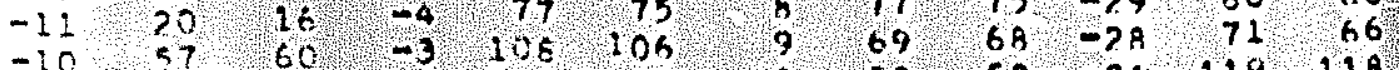

-

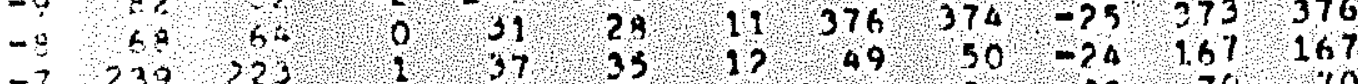

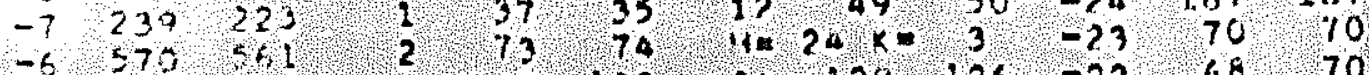

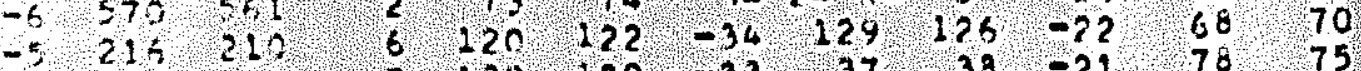

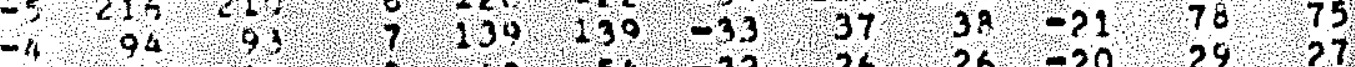

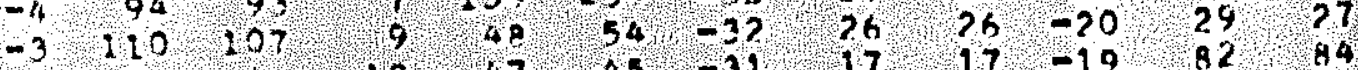

$\begin{array}{llllllllllll}-2 & 76 & 6 & 10 & 47 & 43 & -31 & 17 & 17 & -19 & 82 & 84 \\ -1 & 65 & 62 & 11 & 174 & 177 & -30 & 84 & 87 & -19 & 77 & 80\end{array}$

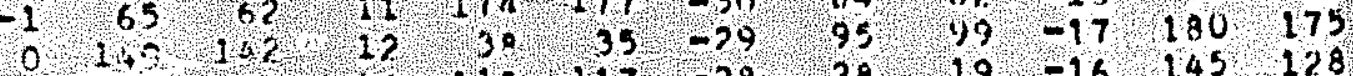

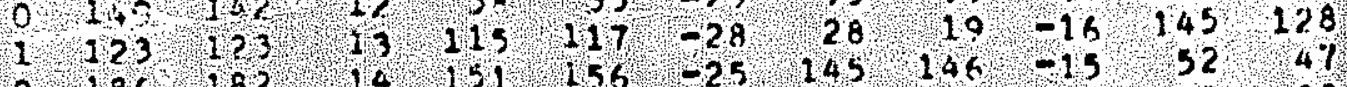

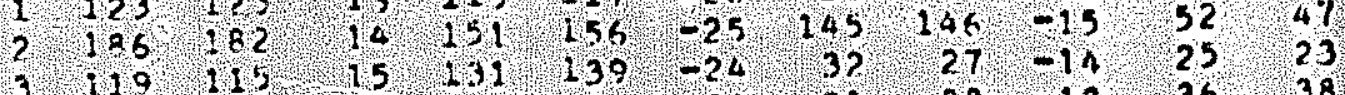

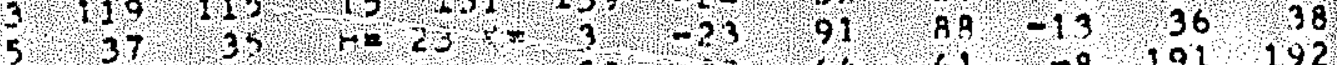

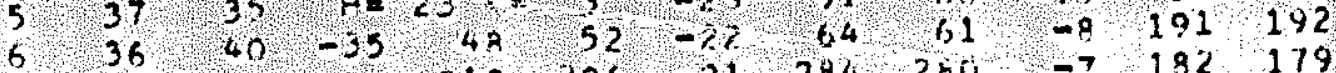

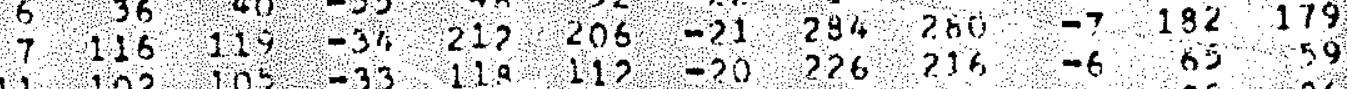

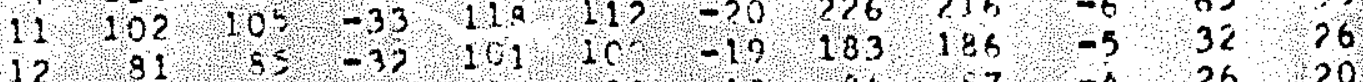

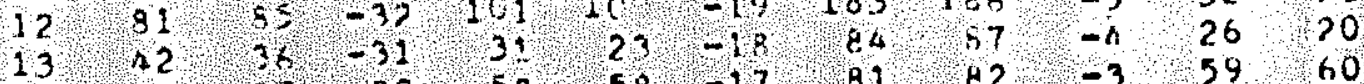

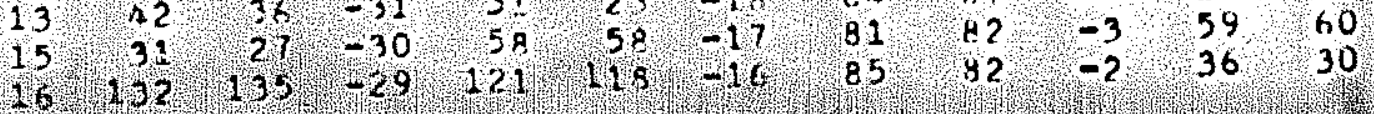




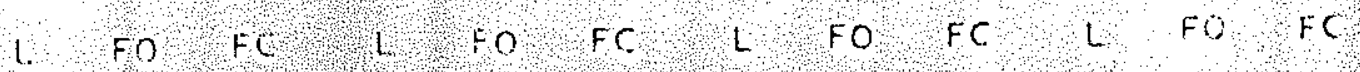

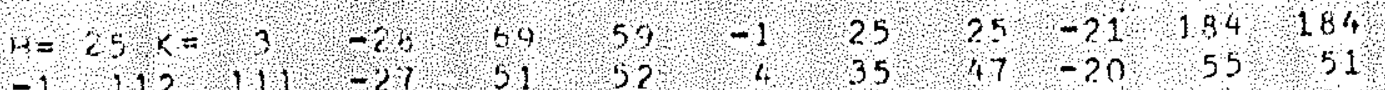

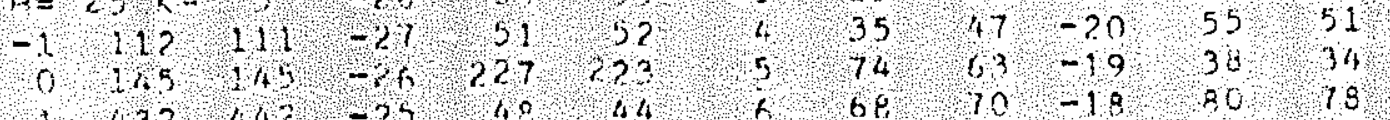

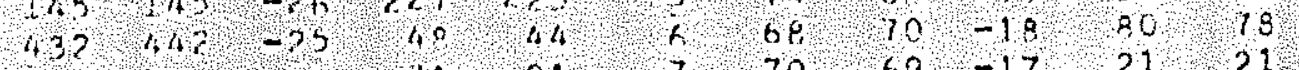

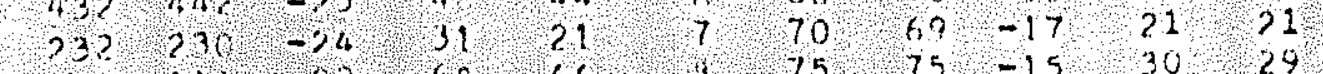

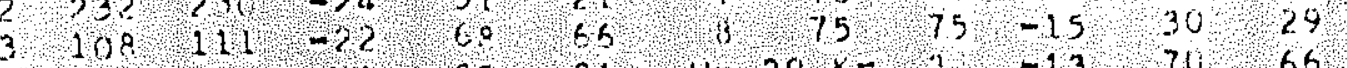

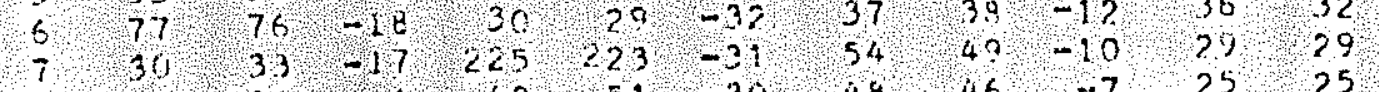

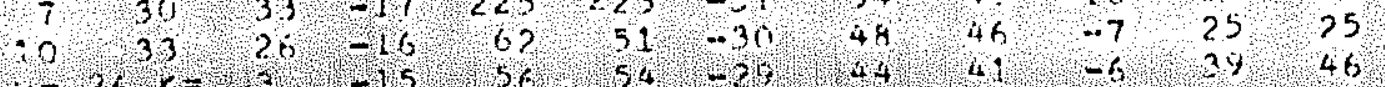

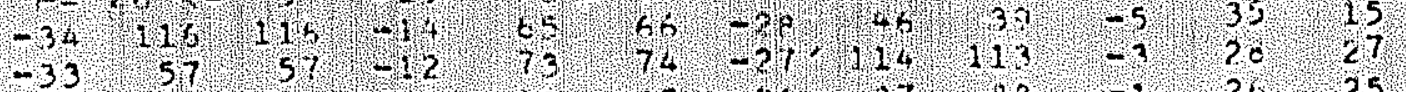

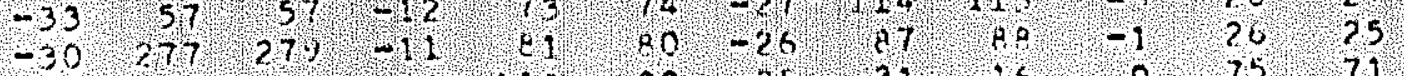

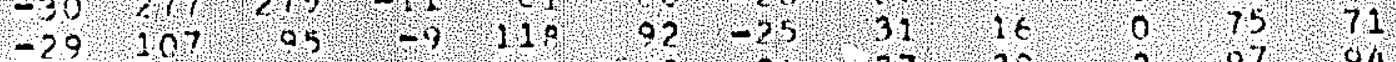

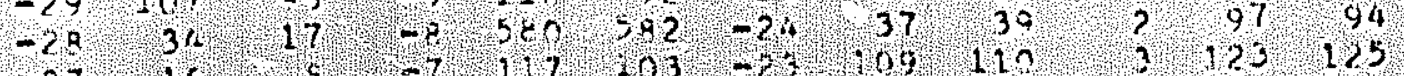

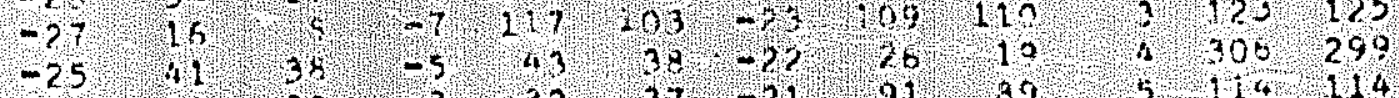

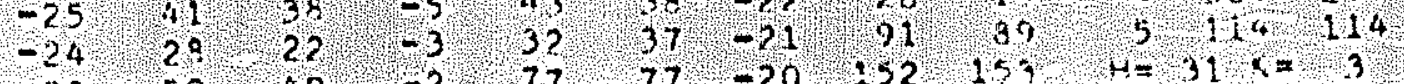

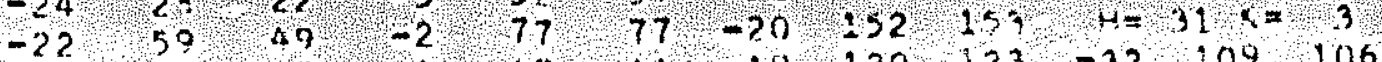

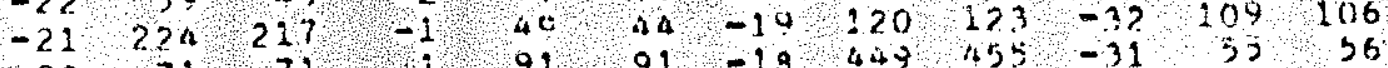

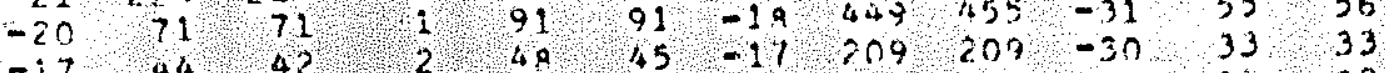

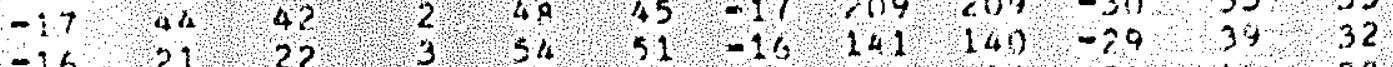

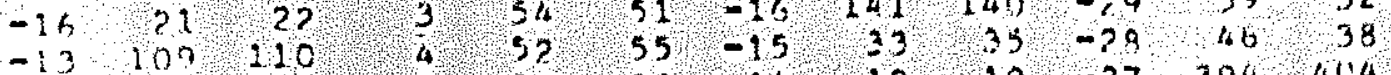

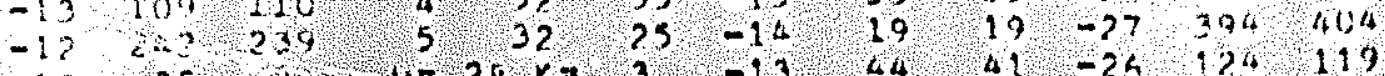

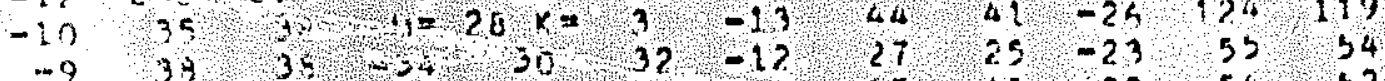

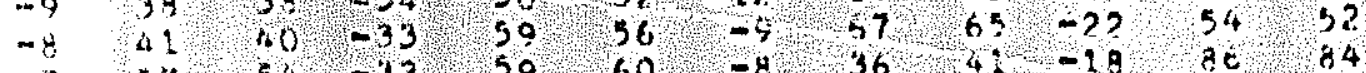

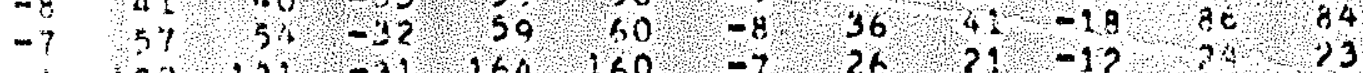

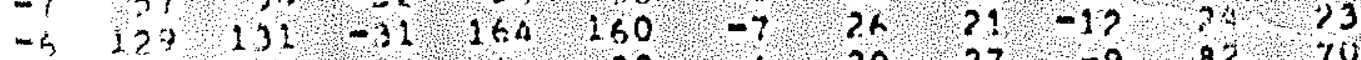

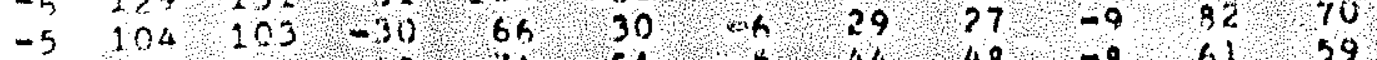

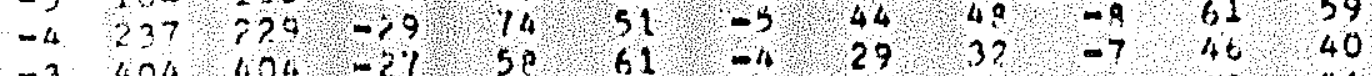

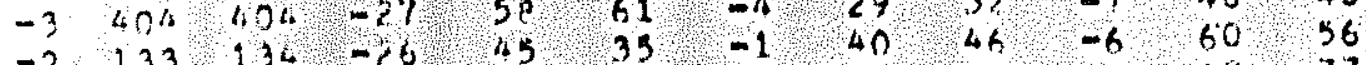

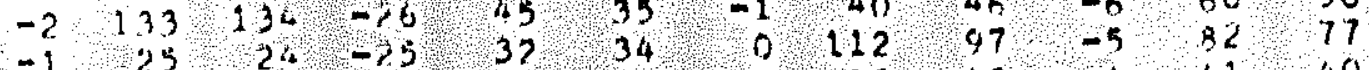

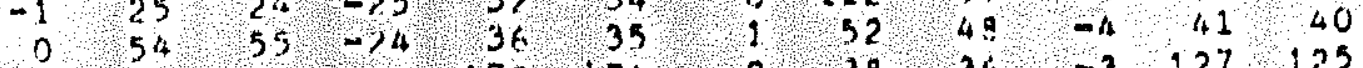

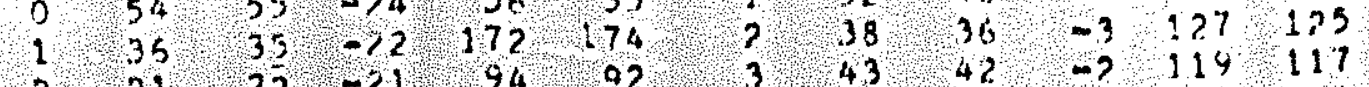

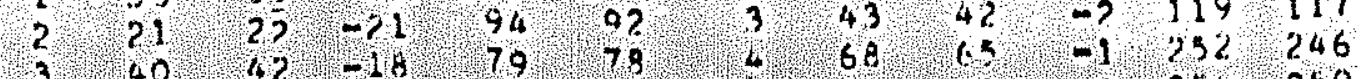

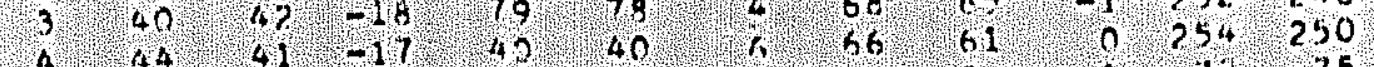

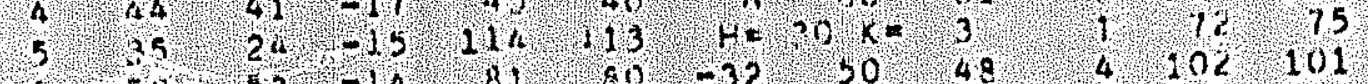

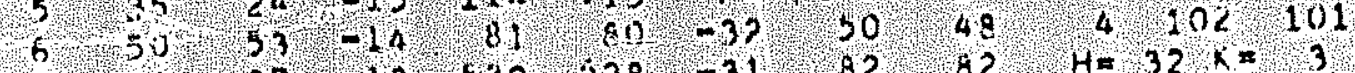

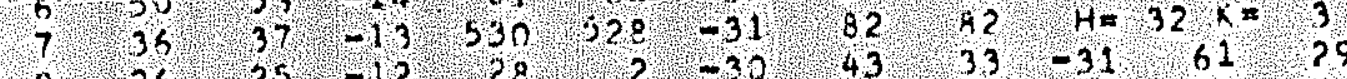

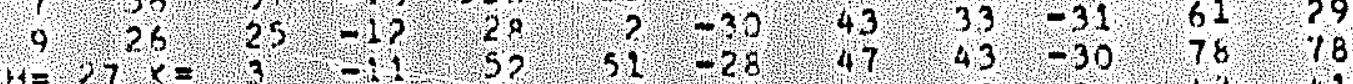

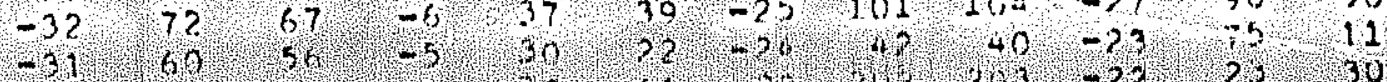

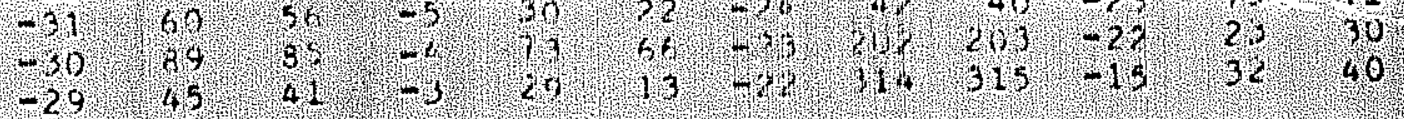




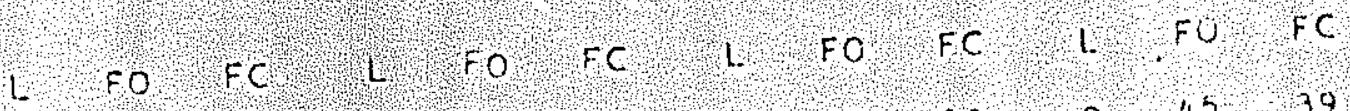

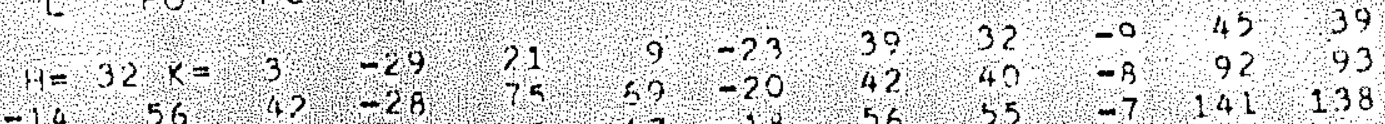

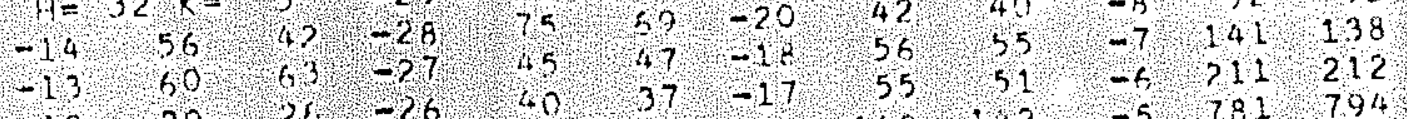

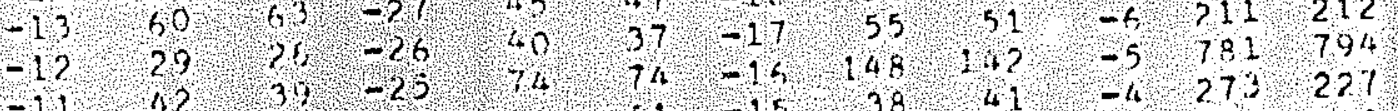

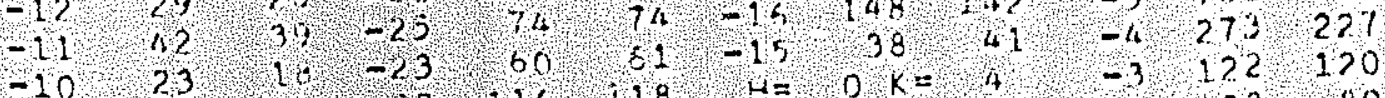

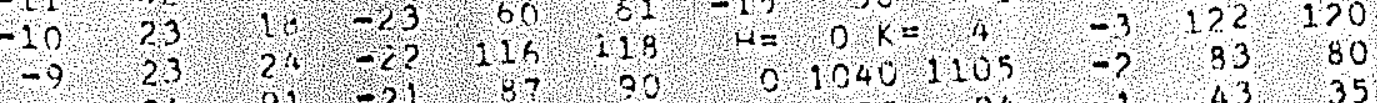

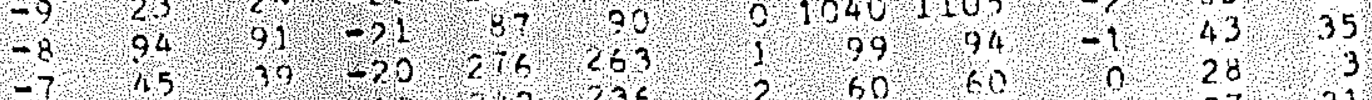

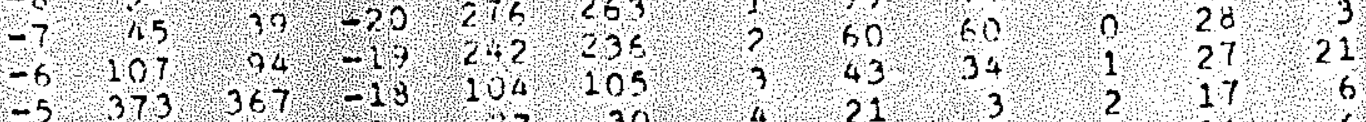

$\begin{array}{lllllllllll}-5 & 373 & 367 & -1 & 1\end{array}$

\begin{tabular}{llllll}
-3 & 23 \\
\hline
\end{tabular}

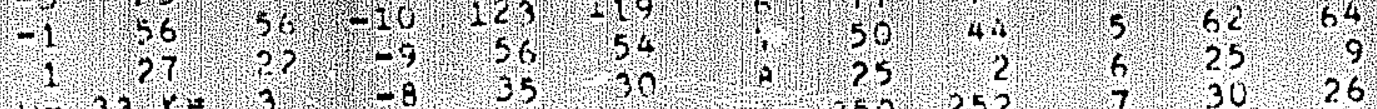

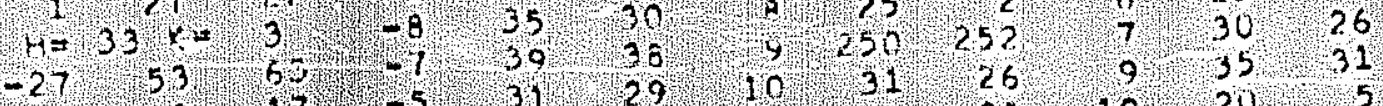

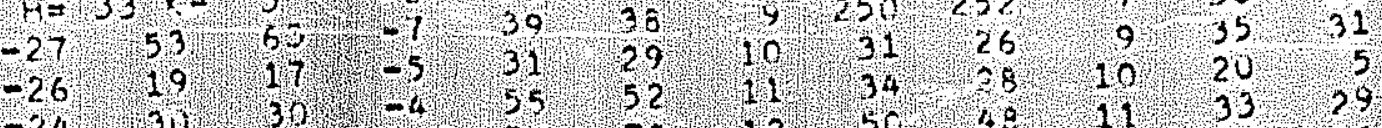

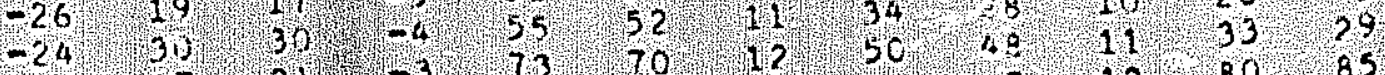

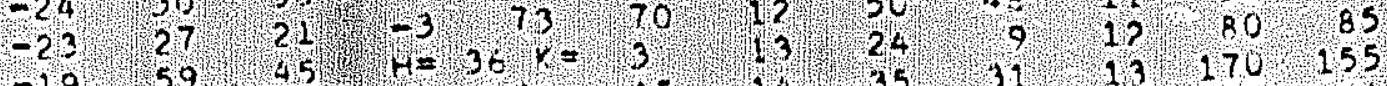

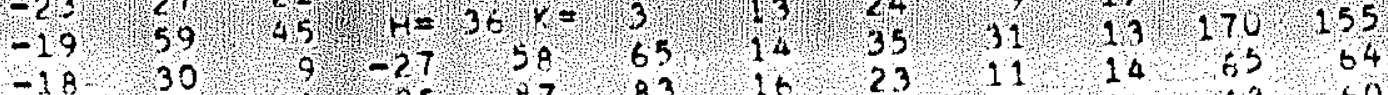

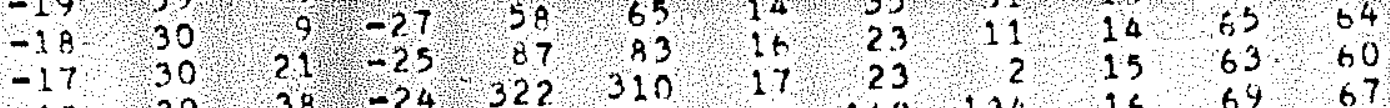

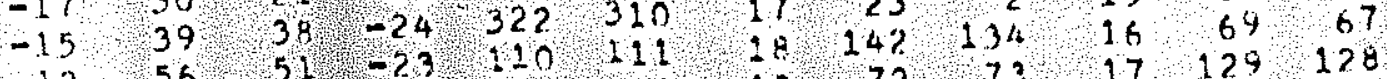

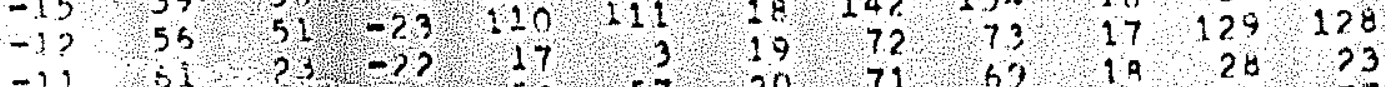

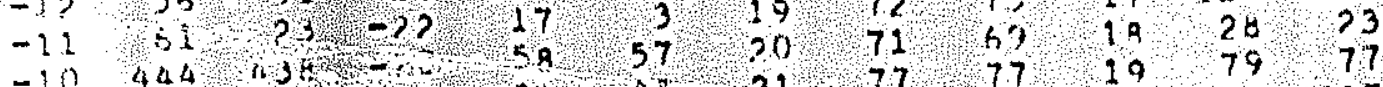

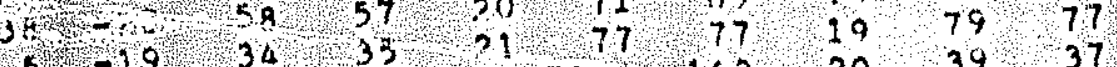

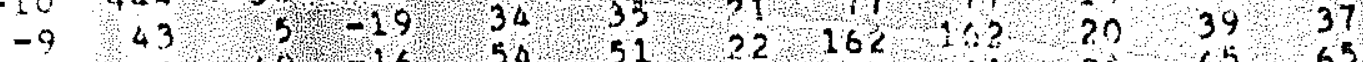

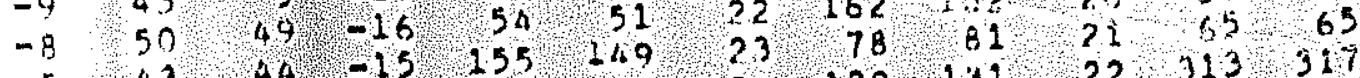

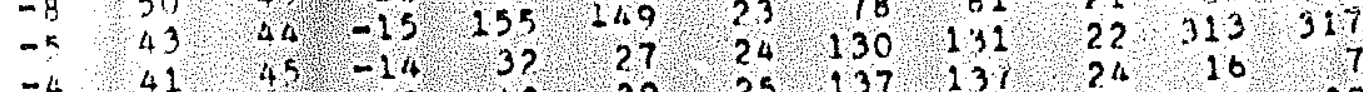

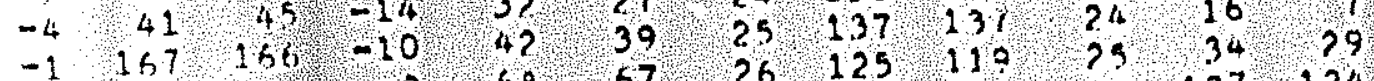

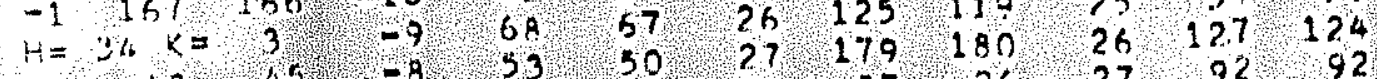

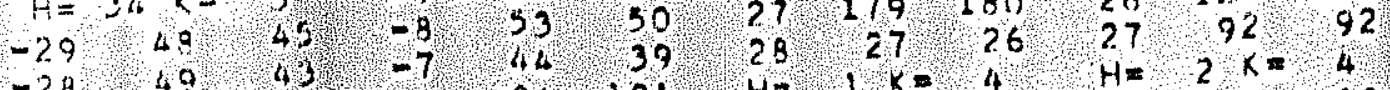

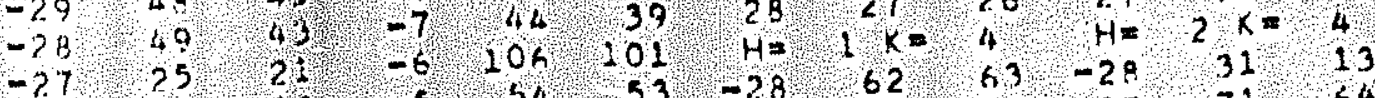

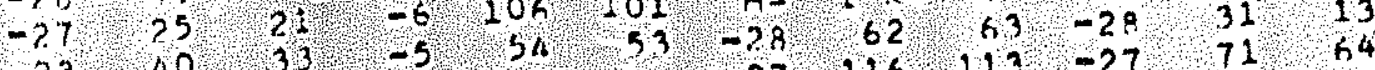

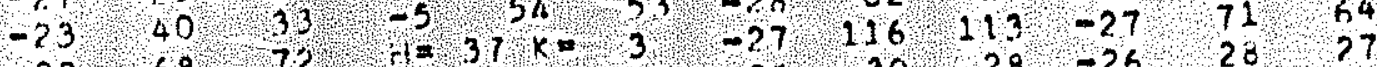

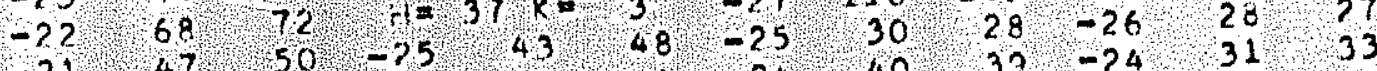

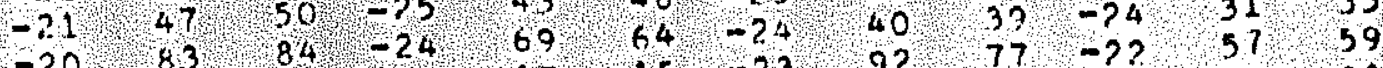

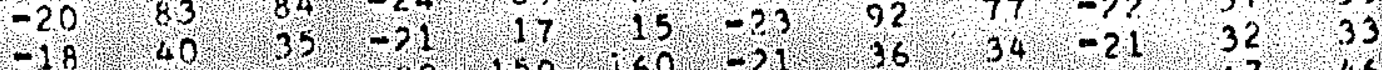

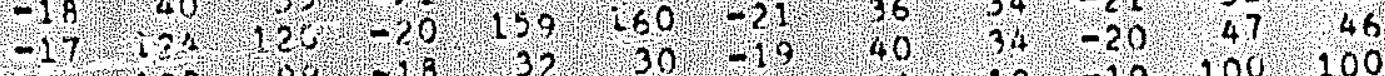

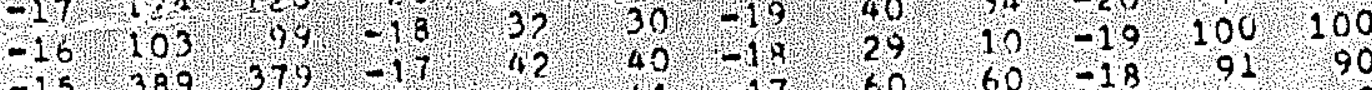

$\begin{array}{lllllllllllll}-15 & 389 & 37 & -17 & 43 & 41 & -17 & 60 & 60 & -18 & 91 & 90\end{array}$

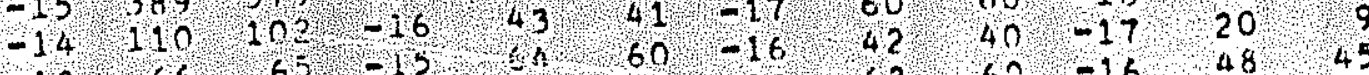

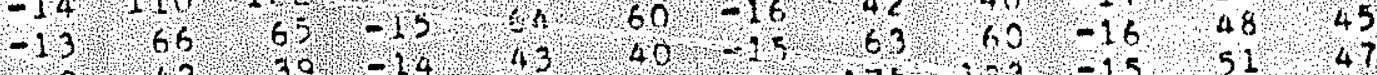

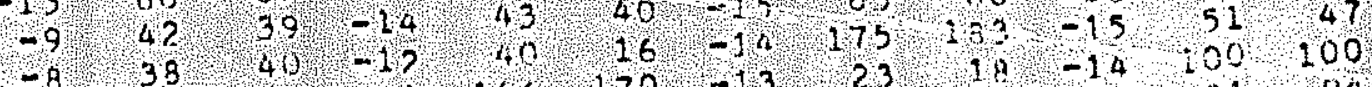

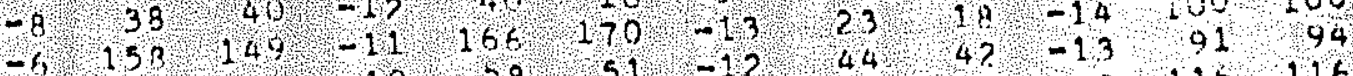

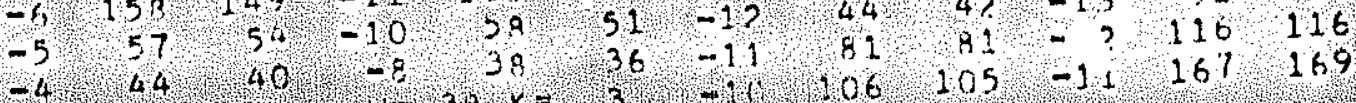

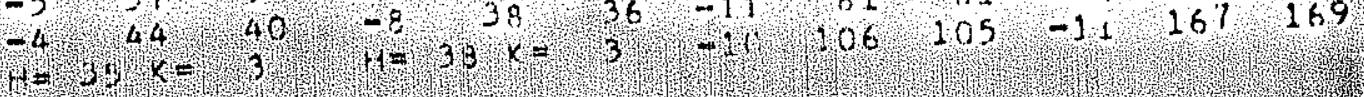




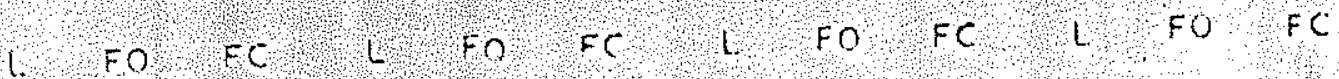

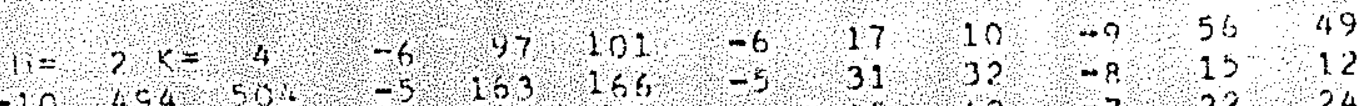

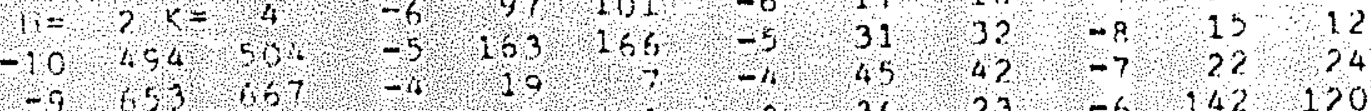

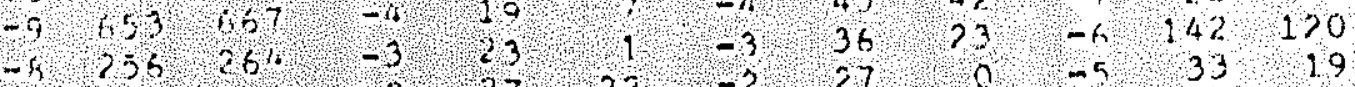

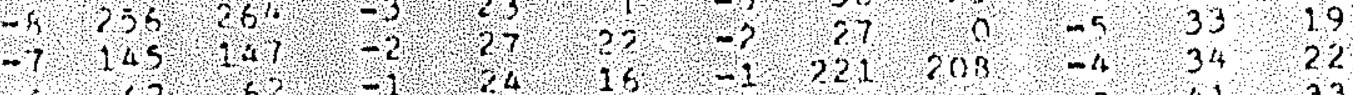

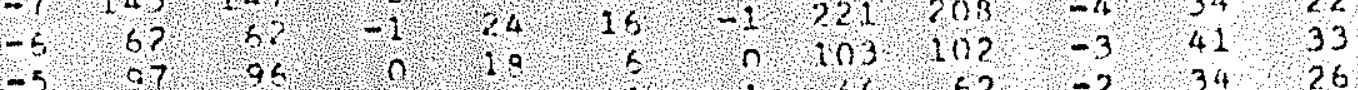

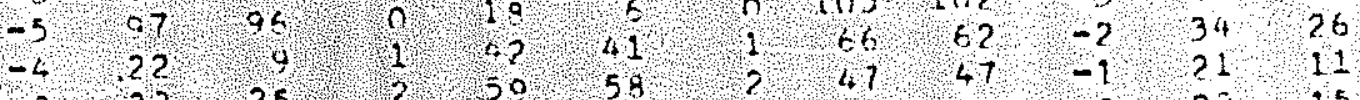

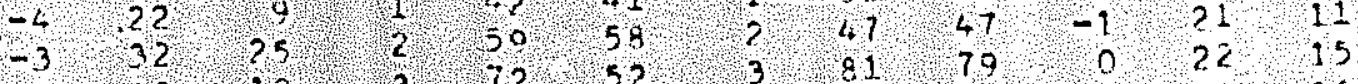

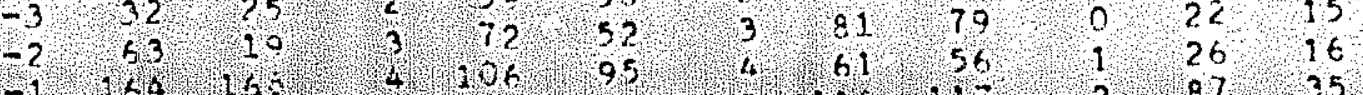

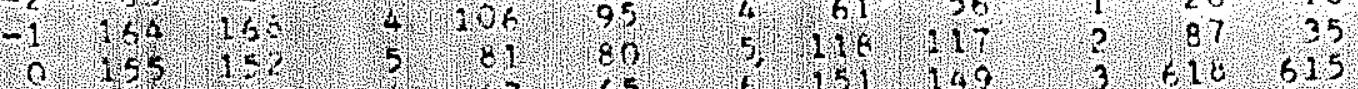

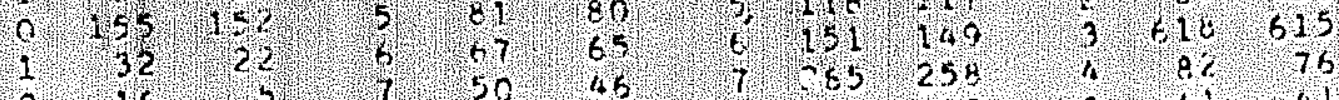

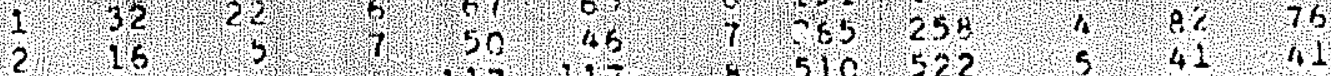

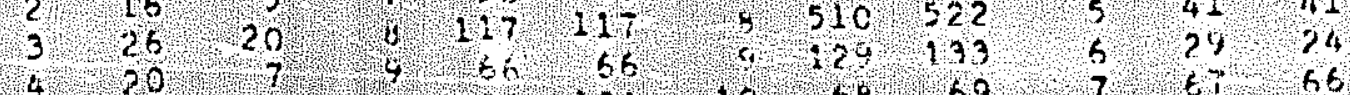

$\begin{array}{lllllllllllll}5 & 16 & 7 & 10 & 135 & 136 & 10 & 68 & 6 ?\end{array}$ ? ?

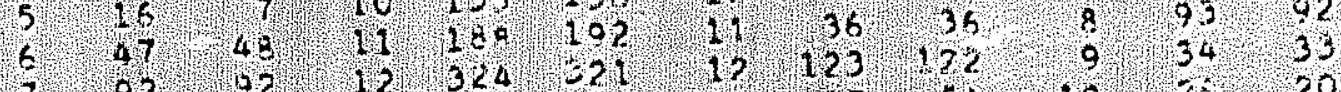

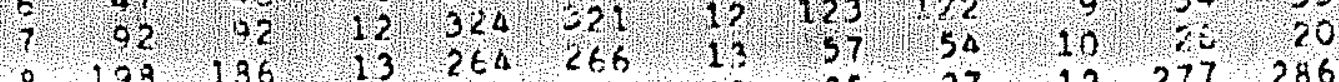

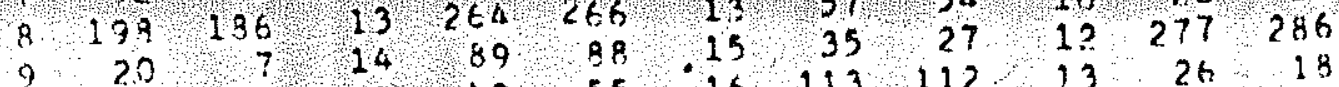

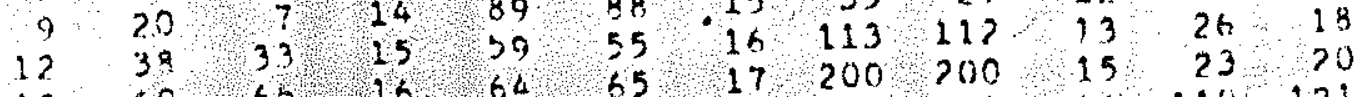

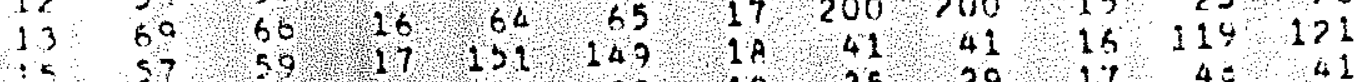

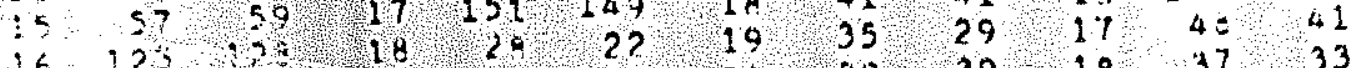

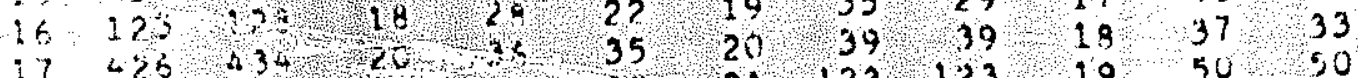

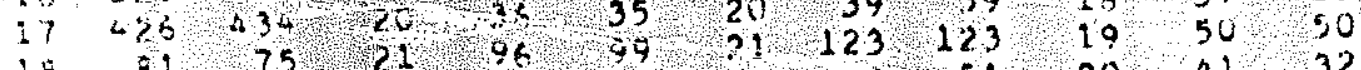

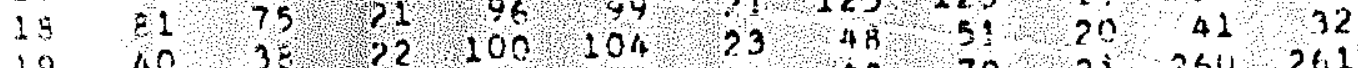

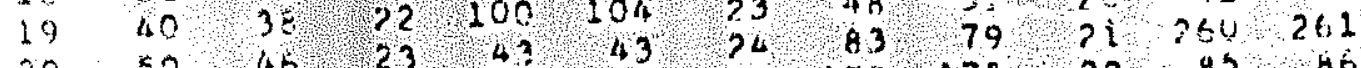

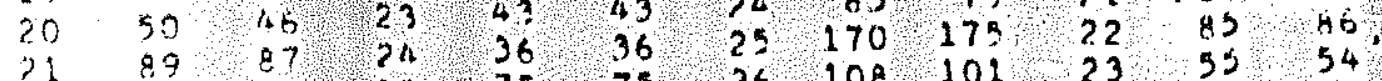

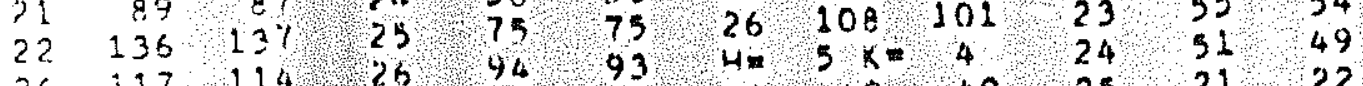

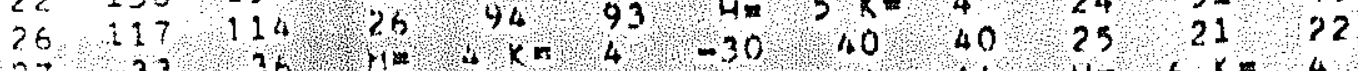

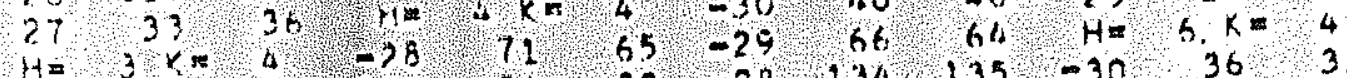

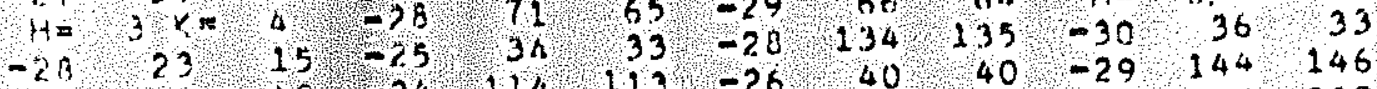

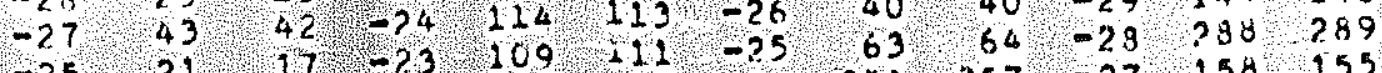

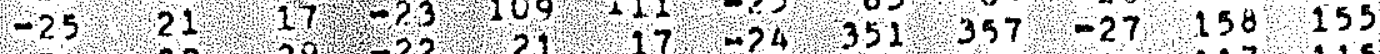

$\begin{array}{lllllllllllll}-24 & 32 & 29 & -22 & 21 & 17 & -23 & 115 & 121 & -26 & 117 & 115\end{array}$

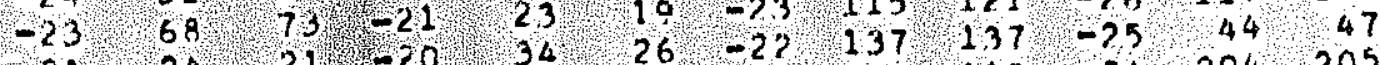

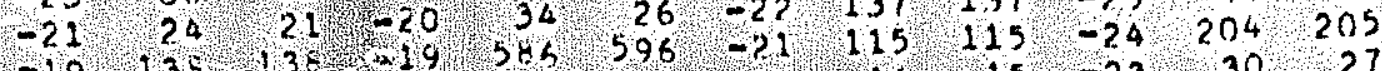

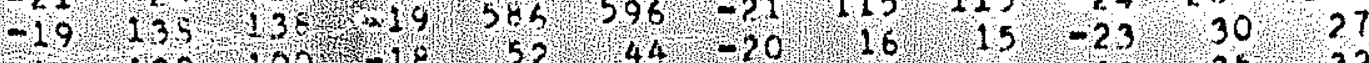

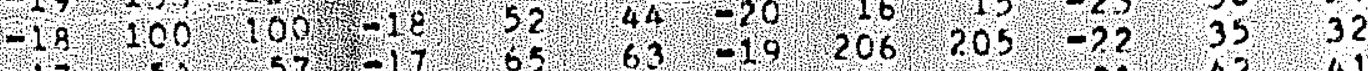

$\begin{array}{lllllllllllll}-17 & 54 & 57 & -17 & 65 & 63 & -19 & 206 & 48 & -21 & 42 & 41\end{array}$

$\begin{array}{llllllllllllll}-16 & 61 & 50 & -16 & 65 & 69 & 5\end{array}$

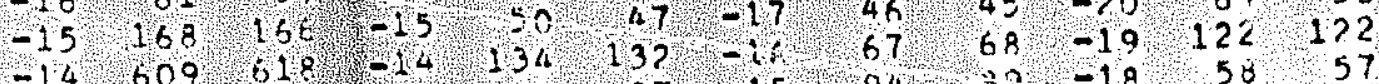

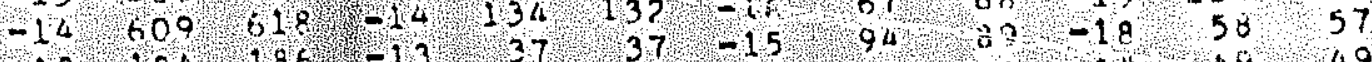

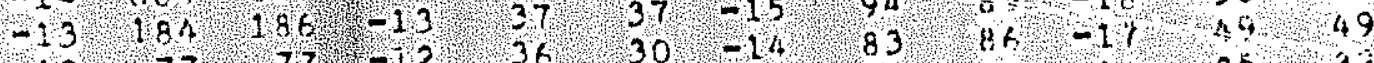

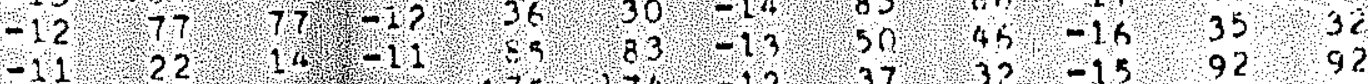

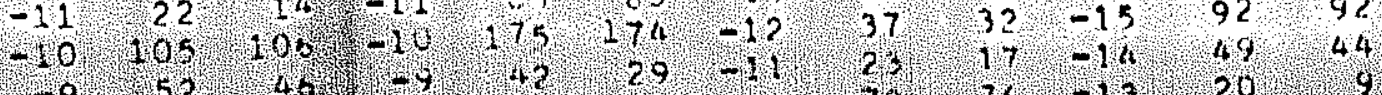

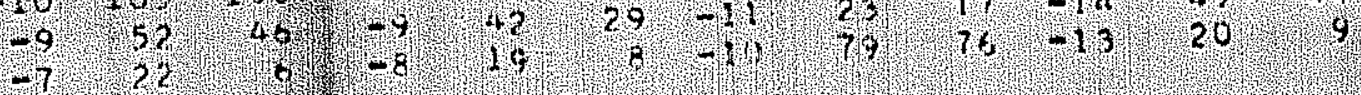




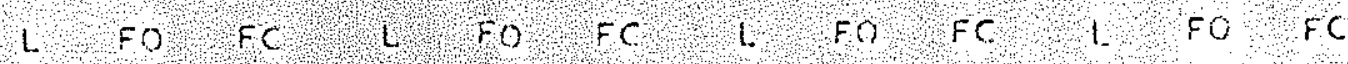

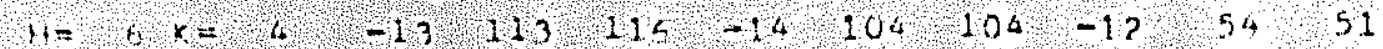

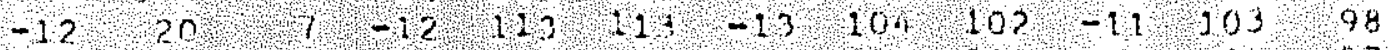

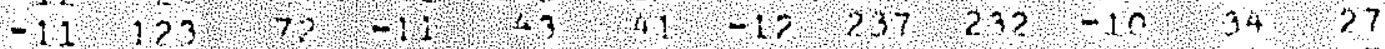

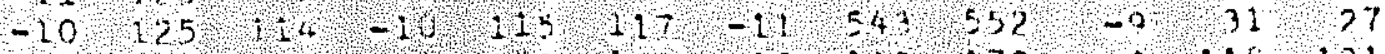

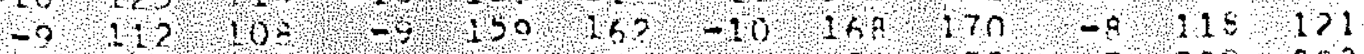

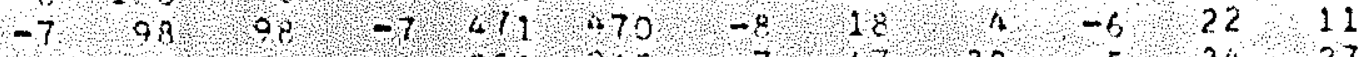

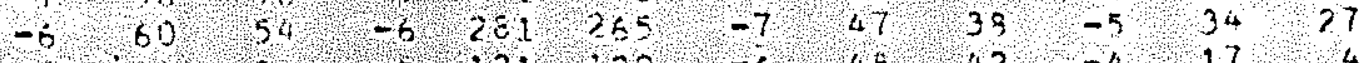

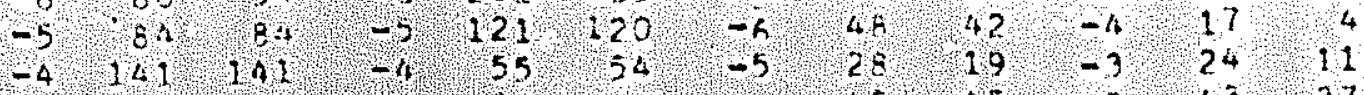

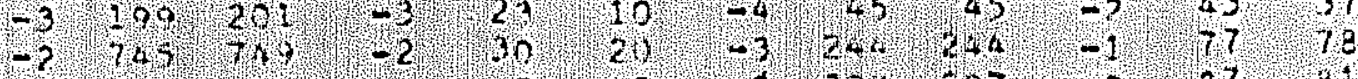

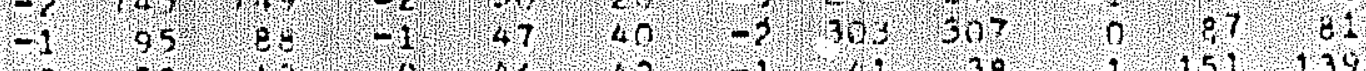

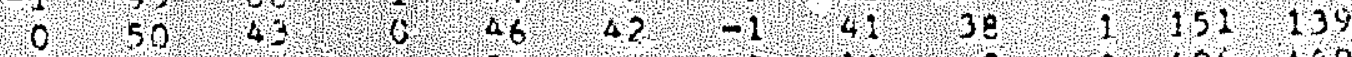

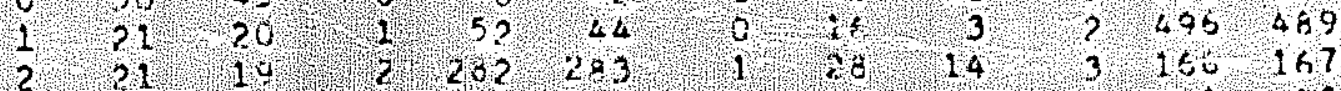

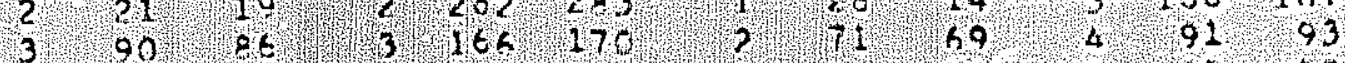

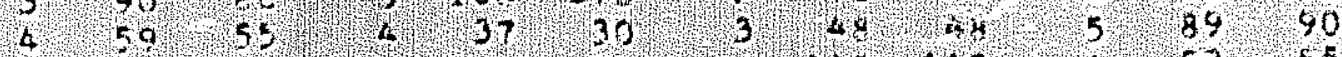

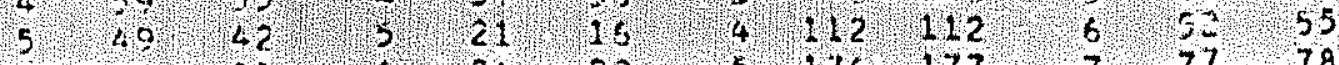

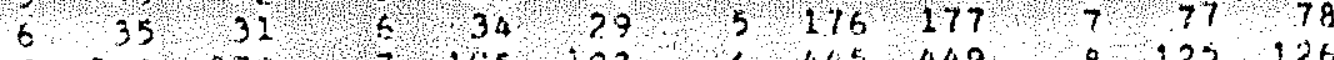

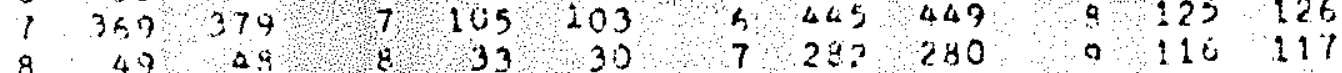

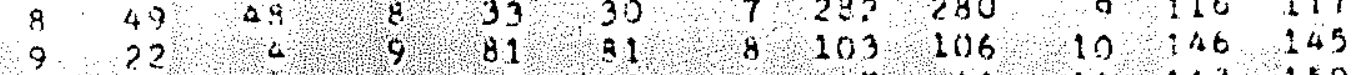

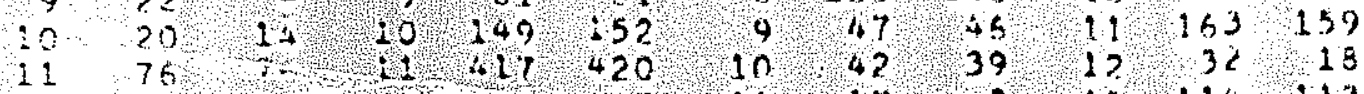

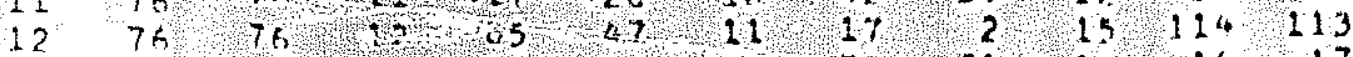

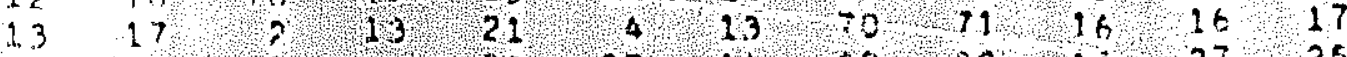

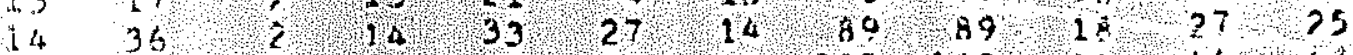

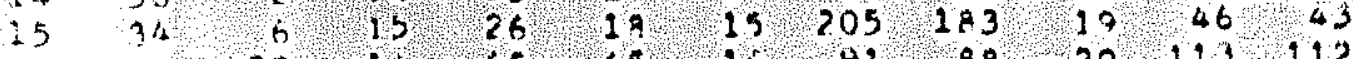

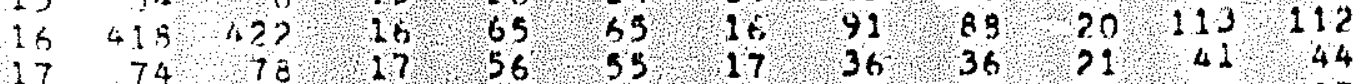

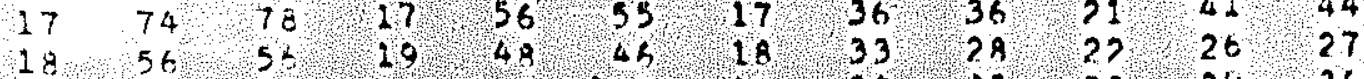

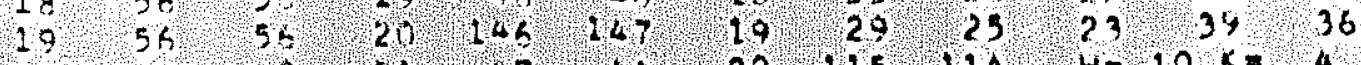

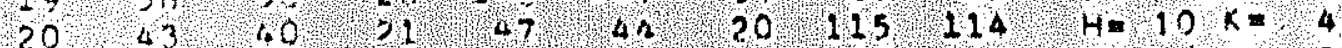

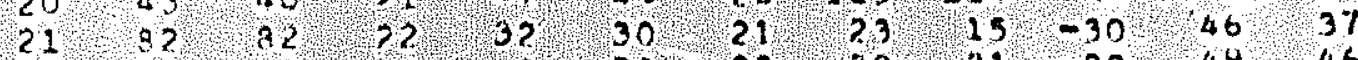

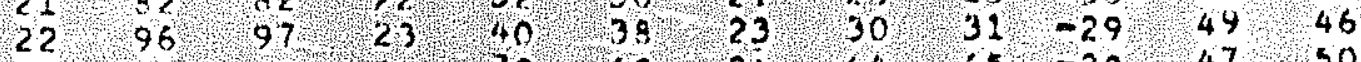

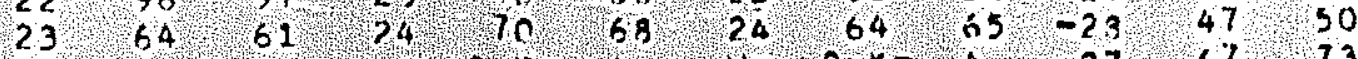

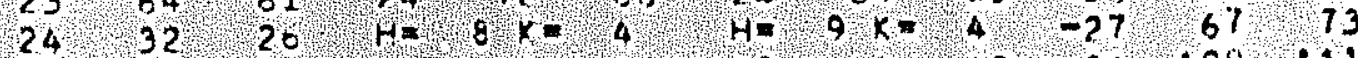

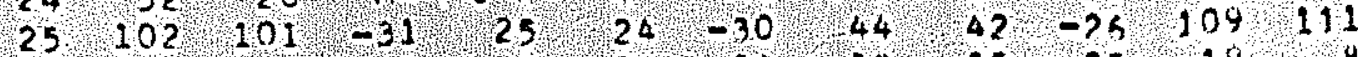

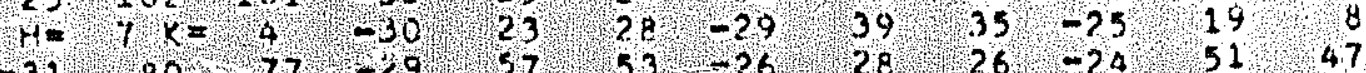

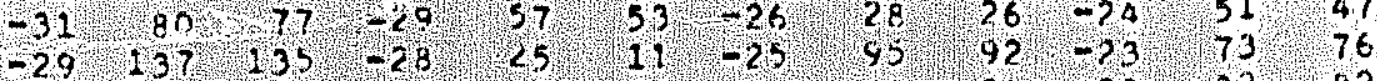

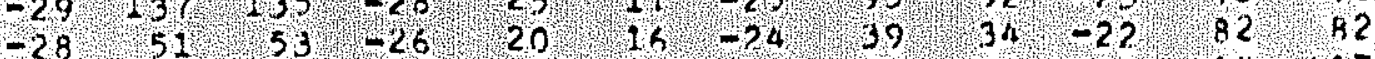

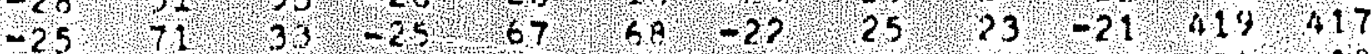

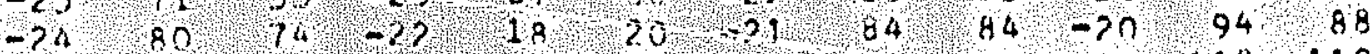

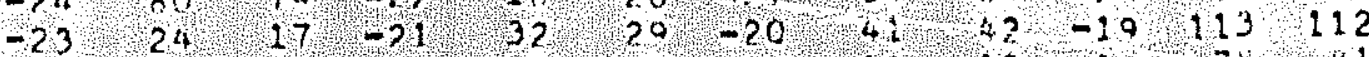

$\begin{array}{llllllllllllll}-22 & 28 & 2 ? & -20 & 155 & 447 & -19 & 19 & 12 & -18 & 7\end{array}$

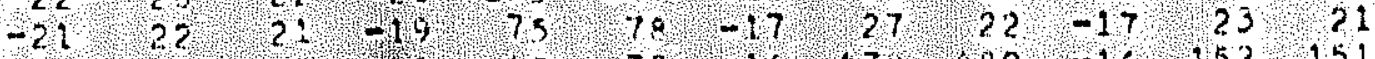

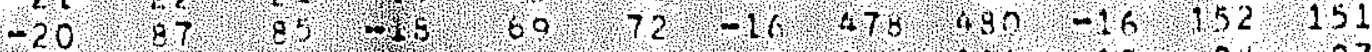

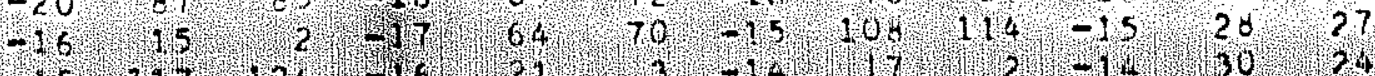

-15
-11 


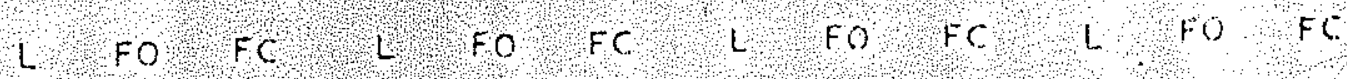

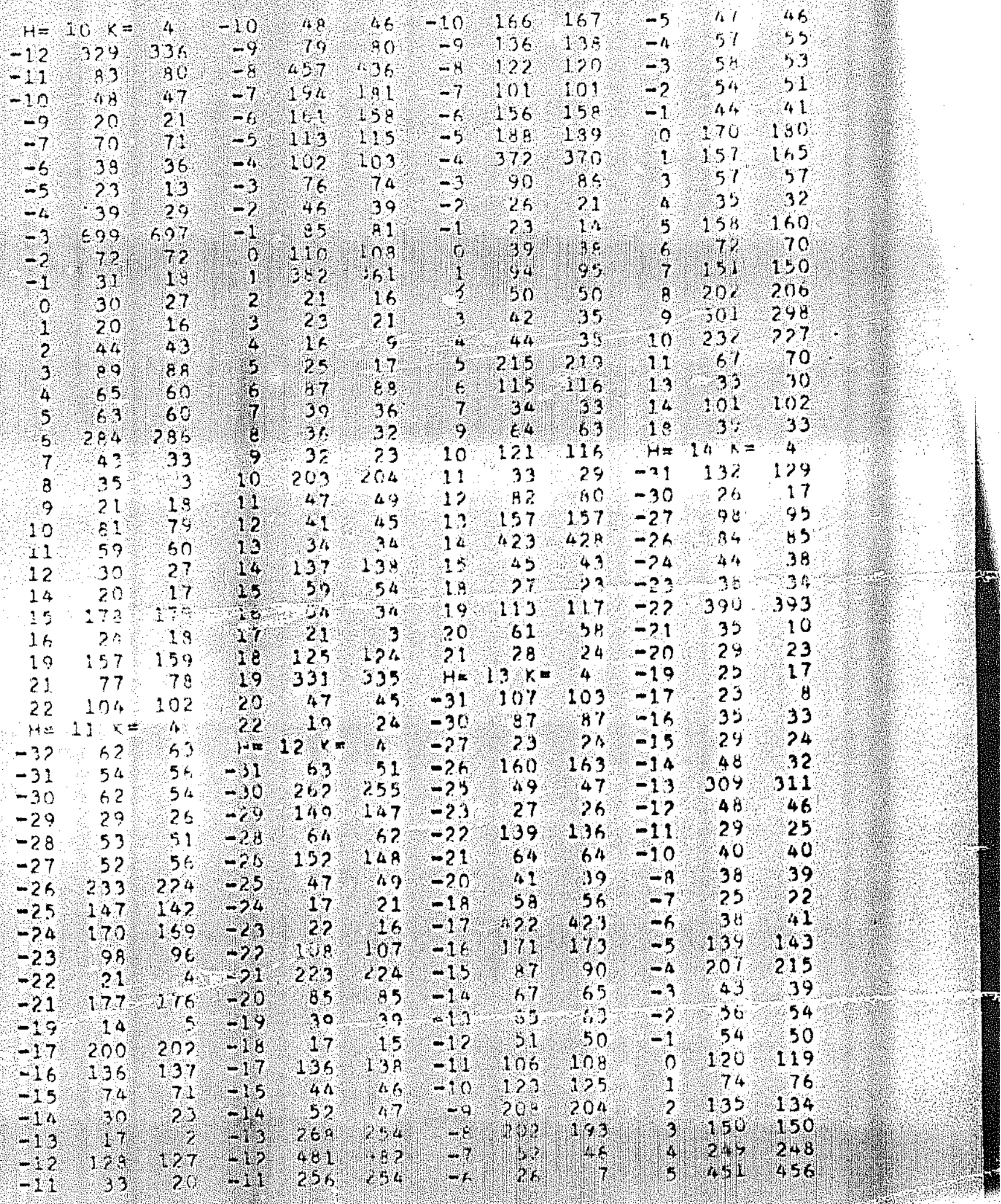




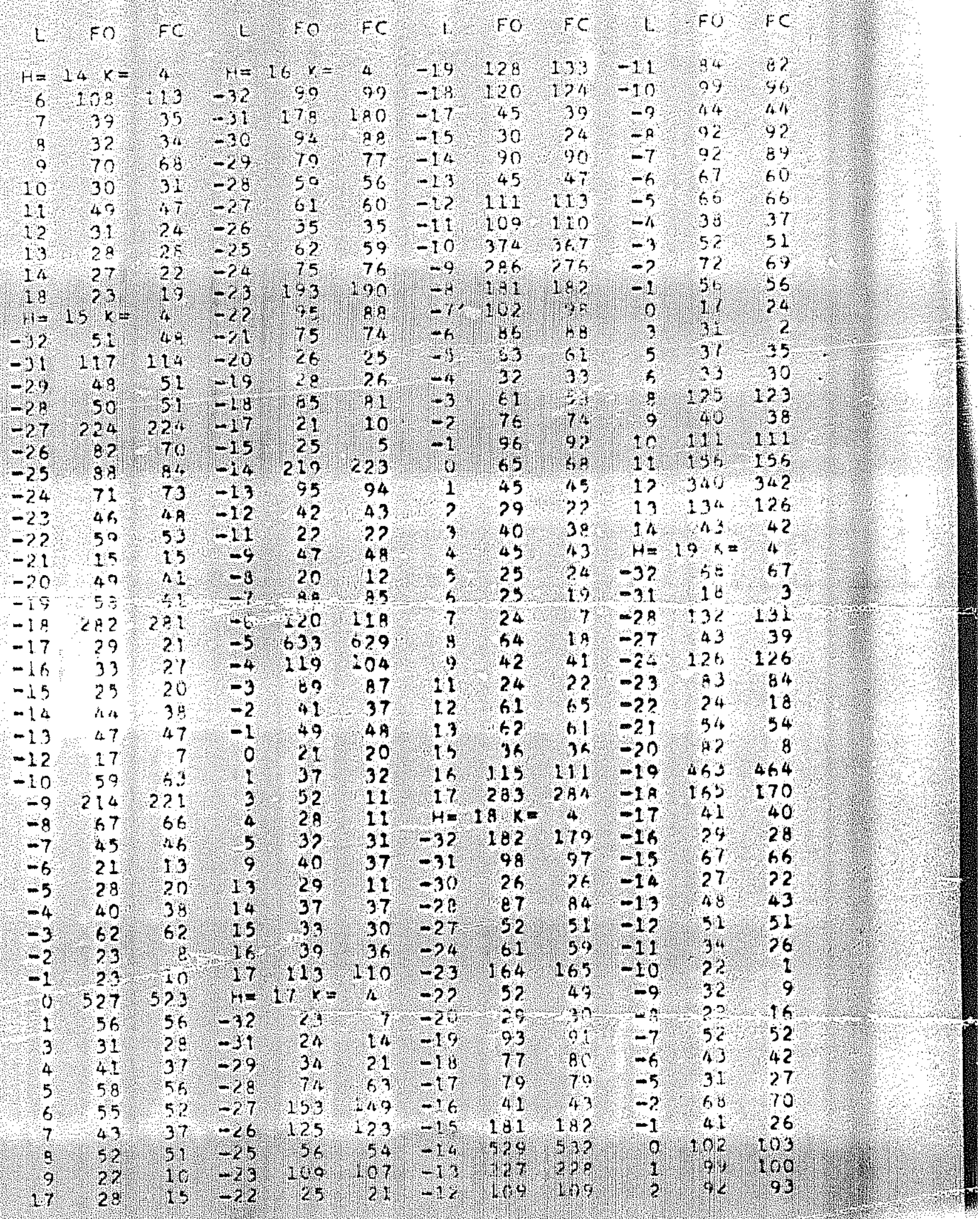




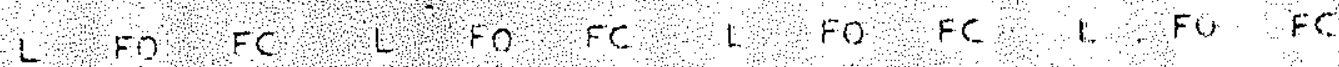

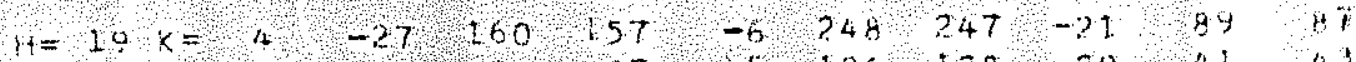

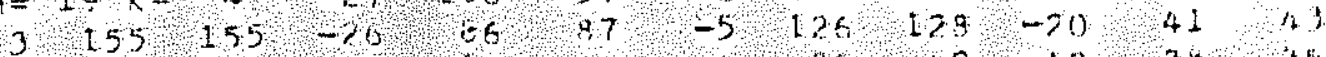

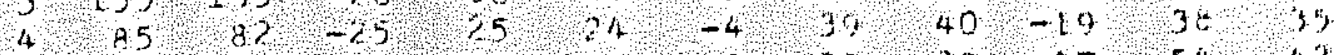

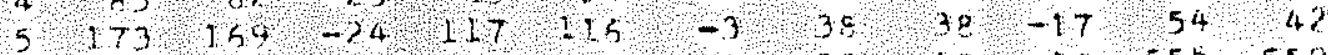

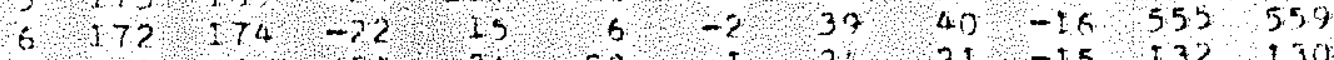

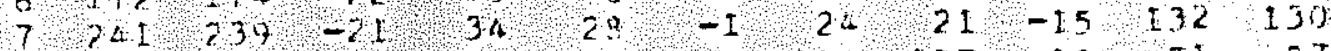

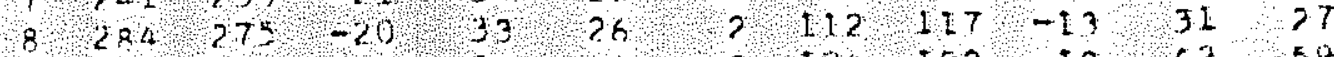

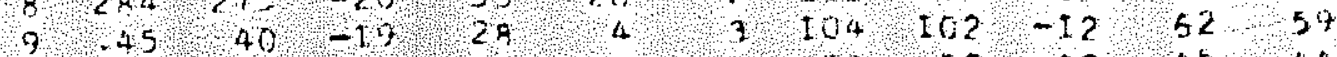

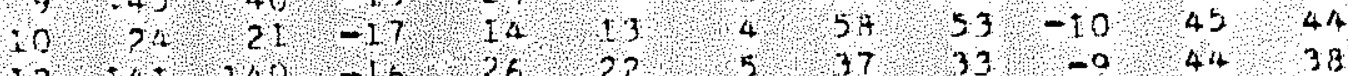

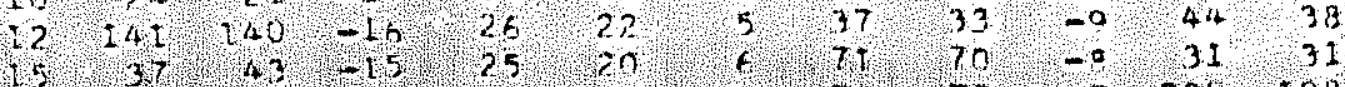

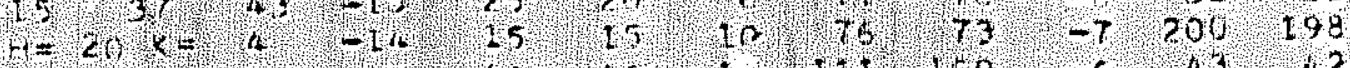

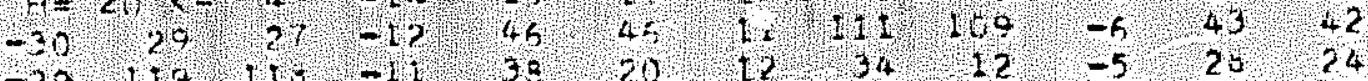

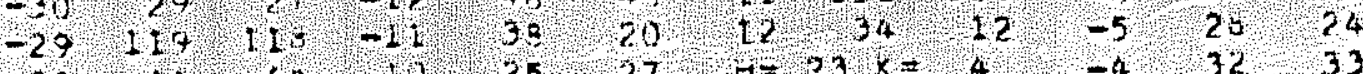

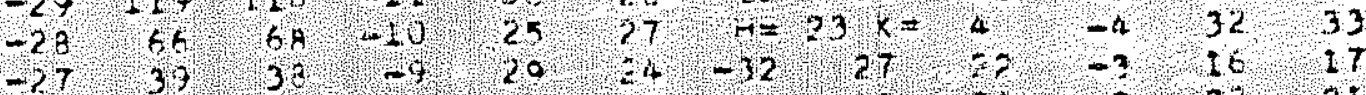

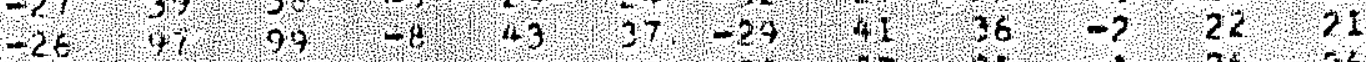

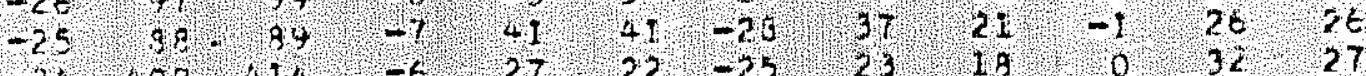

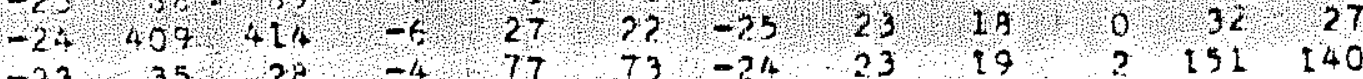

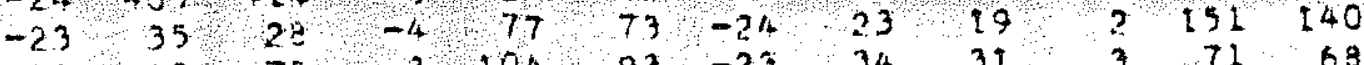

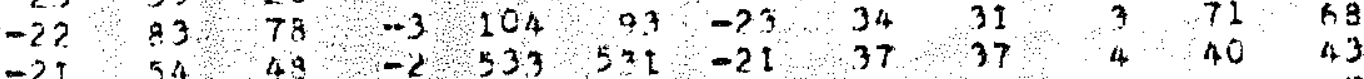

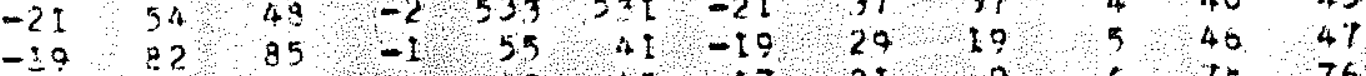

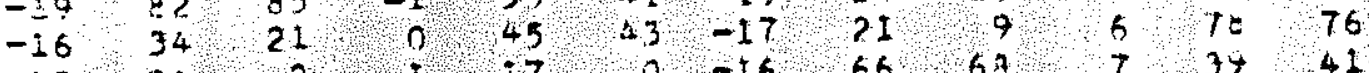

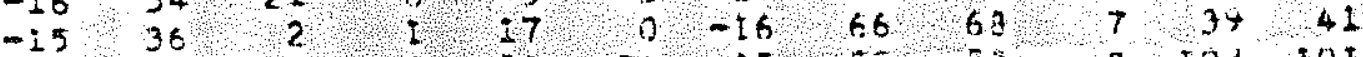

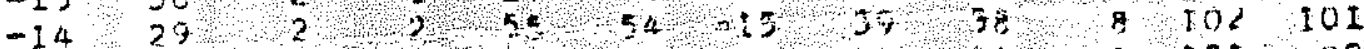

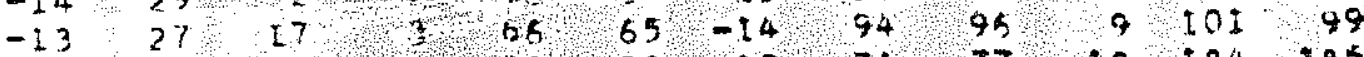

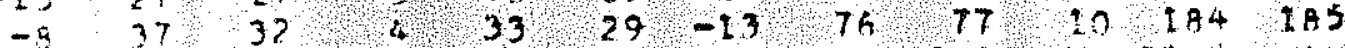

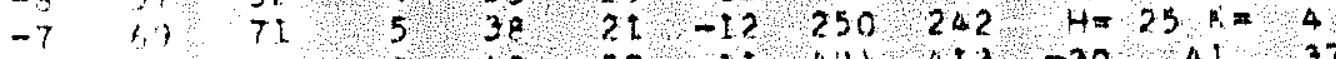

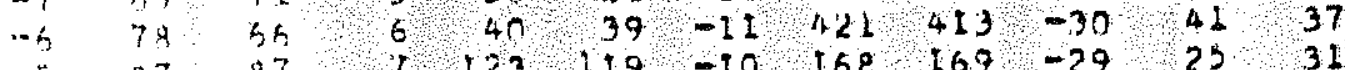

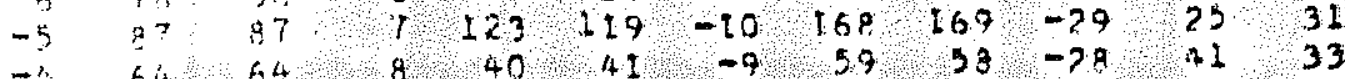

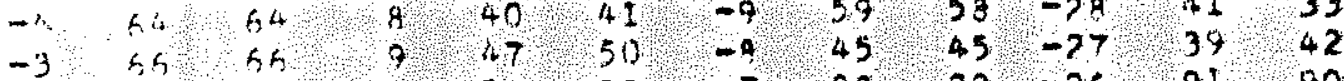

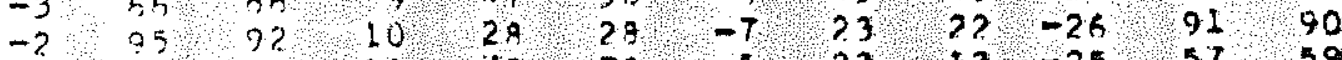

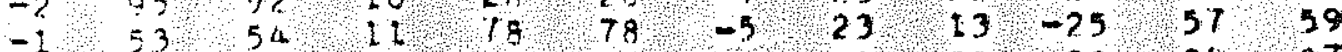

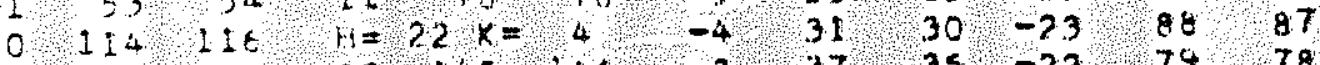

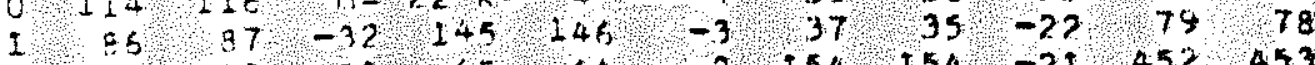

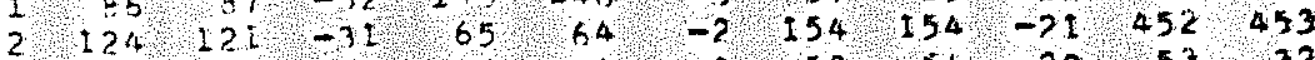

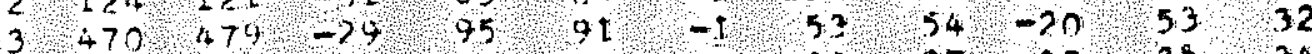

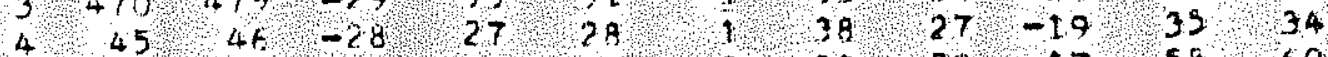

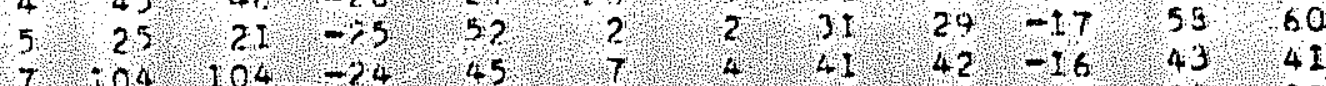

7.49
8

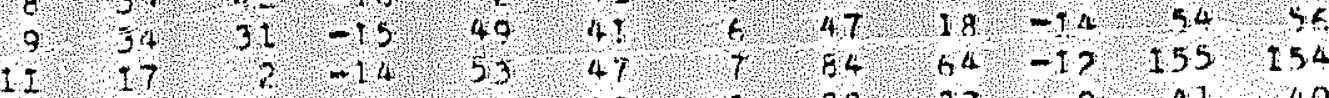

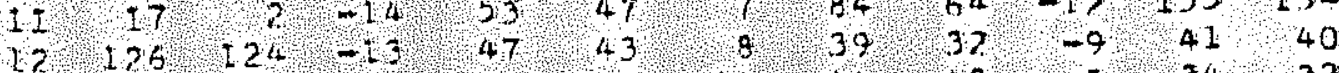

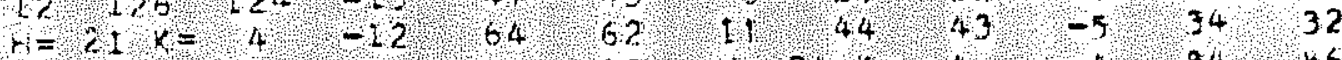

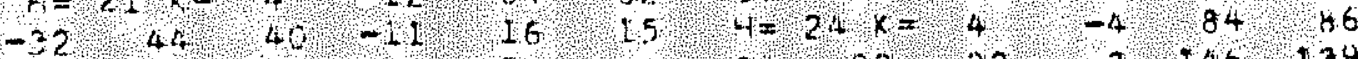

$\begin{array}{llllllllllll}-31 & 93 & 01 & -10 & 56 & 56 & -26 & 22 & 23 & -3 & 146 & 139\end{array}$

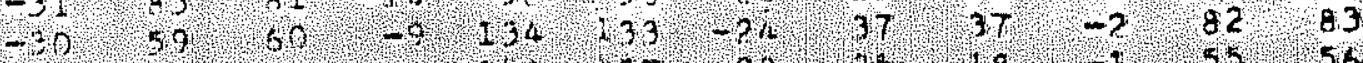

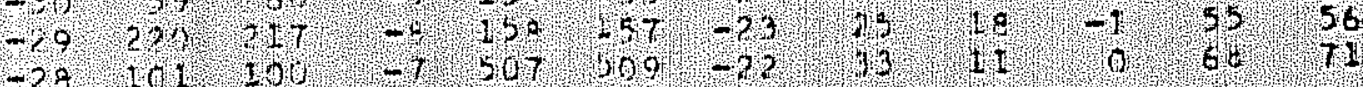




\begin{tabular}{|c|c|c|c|c|c|c|c|c|c|c|c|}
\hline 4 & $F 0$ & $F C$ & L & 50 & $F C$ & 4 & $\mathrm{FO}$ & FC & L & $\mathrm{Fu}$ & $\mathrm{Cc}$ \\
\hline & $25 \times 1=$ & 4 & -15 & 67 & $\mathrm{Ke}$ & -16 & 5.6 & 53 & -2 & 52 & 49 \\
\hline$\frac{1}{2}$ & 1778 & 76 & -14 & 65 & 65 & -14 & 79 & 75 & $a$ & 44 & 47 \\
\hline 3 & 51 & 51 & -10 & 84 & 13 & -13 & 347 & 343 & $H=$ & I? $K=$ & 4 \\
\hline 3 & 107 & 100 & -11 & 56 & 57 & -12 & 177 & 76 & -27 & 151 & 152 \\
\hline 5 & 85 & 41 & -10 & $\angle c$ & 30 & -5 & 53 & 53 & -7.6 & 6,4 & 01 \\
\hline 5 & 110 & $11=$ & -9 & 46 & 44 & -4 & 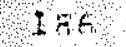 & 189 & -24 & 36 & 39 \\
\hline 6 & 222 & & -8 & 4 & 30 & 0 & 477 & 75 & -23 & & 52 \\
\hline 9 & -44 & 3 & -6 & 70 & 78 & 1 & 25 & 27 & -22 & 40 & 41 \\
\hline 9 & 425 & 21 & -5 & 88 & 83 & 2 & 76 & 79 & $-? 1$ & 37 & 196 \\
\hline$+=$ & $26 \mathrm{r}=$ & 4 & -4 & 311 & 020 & 14? & 69 & 90 & -19 & 153 & 152 \\
\hline 3 & 8.5 & 62 & -3 & 108 & 106 & $4=$ & $30 k=$ & 4 & -19 & 90 & 83 \\
\hline $\begin{array}{r}30 \\
89\end{array}$ & 52 & 46 & -2 & 54 & 54 & 427 & 101 & 102 & -17 & 30 & 79 \\
\hline $\begin{array}{r}-29 \\
-28\end{array}$ & 59 & 57 & 1 & 57 & 69 & -2 & 27 & Sh & -19 & 21 & 31 \\
\hline 28 & IIO & $10 \%$ & 2 & 10 & 12 & -24 & 3.4 & 32 & -13 & 47 & 32 \\
\hline 26 & 105 & 107 & 3 & 29 & 25 & 27 & 61 & 62 & $-1 ?$ & 100 & 97 \\
\hline-26 & 324 & 323 & 4 & $2=$ & $>5$ & -20 & 42 & 59 & -11 & $10 c$ & 103 \\
\hline 25 & $1+5$ & 140 & 4 & 138 & 137 & $-1>$ & .5 & 45 & $-1 n$ & 313 & 11 \\
\hline-24 & 116 & 112 & 6 & 178 & 51 & -18 & 357 & 356 & -0 & 119 & 114 \\
\hline-23 & 37 & 30 & $r=$ & $268=$ & 4. & -47 & 28 & 4 & -8 & 72 & 11 \\
\hline-22 & 23 & 22 & -30 & $(79$ & 60 & -16 & 16 & 11 & -2 & 40 & 40 \\
\hline-21 & 99 & 96 & -29 & 40 & 37 & -14 & 42 & 39 & $\mu=$ & $33 \mathrm{k}=$ & 4 \\
\hline $\begin{array}{l}-20 \\
-17\end{array}$ & 22 & $2 \Omega$ & -27 & 55 & 55 & -12 & 20 & 76 & -24 & 80 & do \\
\hline $\begin{array}{l}-17 \\
-16\end{array}$ & 150 & 254 & -26 & 95 & 22 & -11 & $\begin{array}{l}33 \\
\end{array}$ & 27 & -23 & 120 & $11 ?$ \\
\hline $\begin{array}{l}-16 \\
-10\end{array}$ & 45 & 41 & -25 & 9 & 37 & $1-9$ & 213 & 2.14 & -19 & 54 & 36 \\
\hline $\begin{array}{r}-10 \\
-0\end{array}$ & 62 & 62 & -21 & 53 & 55 & -5 & $\$ B$ & $A A_{2}$ & -19 & 42 & 44 \\
\hline-6 & 28 & 25 & -20 & 55 & 53 & -3 & 35 & 33 & -17 & 81 & 81 \\
\hline $\begin{array}{l}-8 \\
-7\end{array}$ & $1+6$ & 120 & -1 & 27 & 24 & -1 & 44 & 33 & -25 & 53 & 51 \\
\hline $\begin{array}{l}-7 \\
-5\end{array}$ & 58 & 43 & 18 & -5 & 1 & 0 & 319 & 315 & $-i 5$ & 139 & 172 \\
\hline $\begin{array}{l}-5 \\
-4\end{array}$ & 37 & 35 & -17 & 229 & 123 & 8 & 80 & 200 & -14 & 234 & 273 \\
\hline $\begin{array}{l}-4 \\
x-3\end{array}$ & 31 & 31 & -16 & 97 & 94 & 2 & 436 & 28 & -13 & 124 & 139 \\
\hline $\begin{array}{c}-1 \\
-\frac{3}{2}\end{array}$ & 65 & 53 & -15 & 53 & 53 & $\mathrm{H}=$ & $31 \mathrm{k}=$ & 4 & $-1 ?$ & 34 & 195 \\
\hline-1 & 19 & 12 & -14 & $0 ?$ & 62 & -24 & 82 & 80 & -11 & $A B$ & 47 \\
\hline 0 & 56 & 15 & -13 & 30 & 37 & -27 & 31 & 25 & -10 & 2 & 24 \\
\hline 1 & 301 & 312 & $-1 ?$ & 45 & $4 ?$ & -36 & 47 & 41 & -6 & 52 & 46 \\
\hline$?$ & 33 & 28 & -11 & 106 & 106 & -23 & 87 & 84 & -7 & 49 & 49 \\
\hline 3 & 57 & 34 & -10 & 97 & 107 & -34 & 278 & 78 & -6 & 130 & 130 \\
\hline$\frac{4}{5}$ & 23 & 26 & -9 & 230 & 239 & -23 & 267 & 264 & -5 & 54 & 60 \\
\hline 5 & 68 & 67 & -9 & 242 & 281 & -27 & 90 & A० & $4=$ & $34 \mathrm{~K}=$ & 4 \\
\hline 16 & 51 & $5 ?$ & -7 & 89 & 89 & -21 & 50 & 52 & -24 & $\left(\begin{array}{l}36 \\
75\end{array}\right.$ & 29 \\
\hline $\begin{array}{r}1= \\
-31\end{array}$ & $27 x=$ & 4. & -9 & 46 & 44 & -19 & 57 & 48 & $\begin{array}{l}-23 \\
-23\end{array}$ & 73 & $\frac{74}{4}$ \\
\hline-30 & 134 & 13 & 0 & 10 & 112 & -18 & 16 & 20 & -22 & $\begin{array}{l}54 \\
50\end{array}$ & 33 \\
\hline-89 & 210 & 208 & 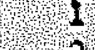 & 236 & 139 & -17 & 41 & 51 & $\begin{array}{r}-20 \\
-19\end{array}$ & & 37 \\
\hline-28 & $\begin{array}{r}79 \\
59\end{array}$ & 135 & 2 & 3 & 32 & -89 & 53 & 180 & -19 & 320 & 314 \\
\hline-26 & $\begin{array}{r}59 \\
126\end{array}$ & 59 & 4 & 82 & 8 & -74 & 187 & 188 & -19 & 27 & 85 \\
\hline-25 & 128 & $\begin{array}{r}129 \\
34\end{array}$ & 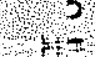 & $6, \quad \mathrm{r}=$ & 60 & -13 & 44 & $\begin{array}{l}48 \\
40\end{array}$ & -13 & 57 & 36 \\
\hline-24 & 17 & 119 & -26 & $\mathrm{sec}$ & 6 & -11 & 38 & 32 & -12 & 42 & 38 \\
\hline$-2 z$ & 8 I & 80 & -25 & 37 & 37 & -9 & 27 & 2 & -11 & 51 & 64 \\
\hline-21 & 57 & 53 & -22 & 49 & 02 & -7 & 83 & 80 & -10 & 103 & 23 \\
\hline-2 & 43 & 35 & -21 & 105 & 99 & -6 & 100 & 104 & $r=$ & $35 k=$ & 4 \\
\hline-17 & 42 & 45 & -2 & 21 & 7 & -5 & 347 & 133 & -22 & $1 \times 54$ & 49 \\
\hline 8 & 31 & 28 & -1 & 24 & 2 & 74 & (2) & 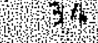 & $2+1$ & 26 & 3 \\
\hline
\end{tabular}




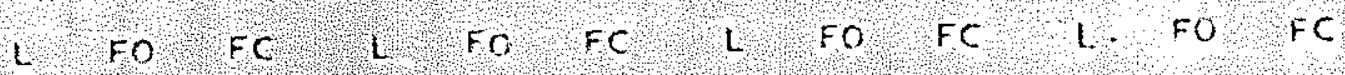

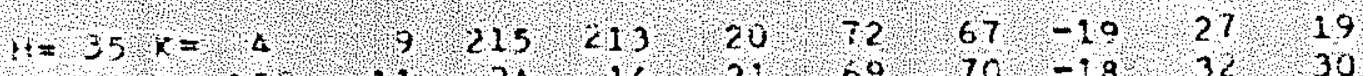

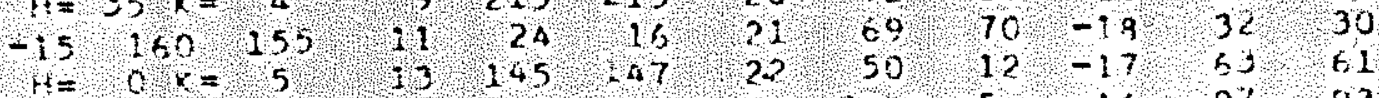

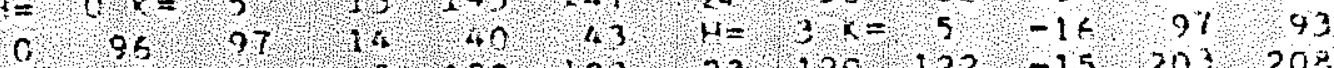

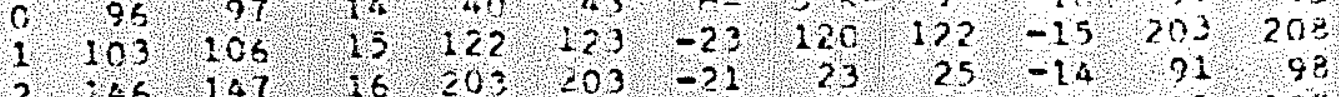

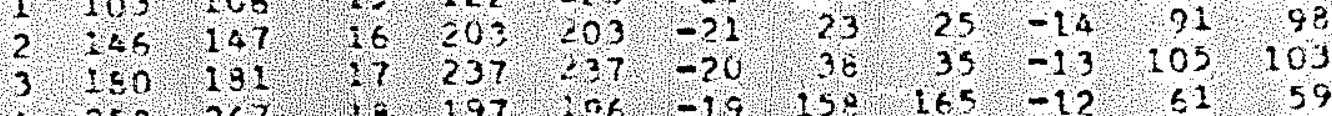

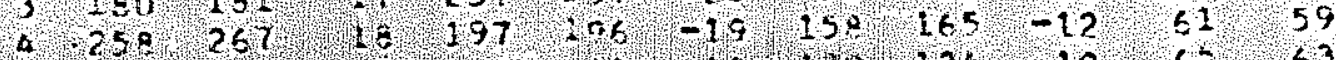

5. 111 1 113 .

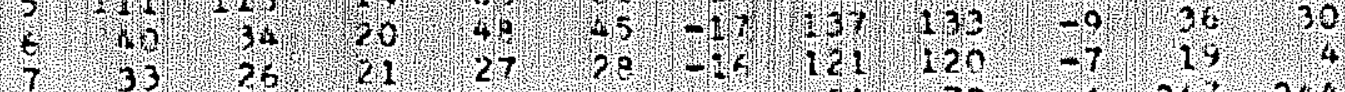

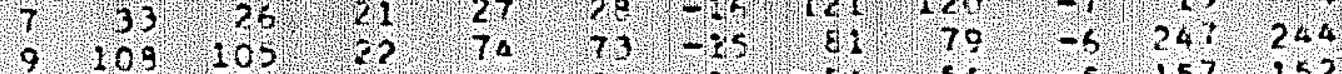

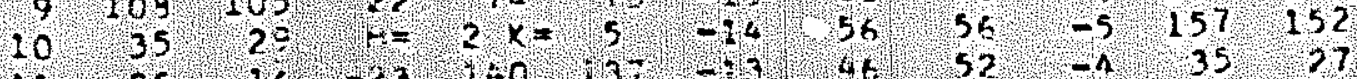

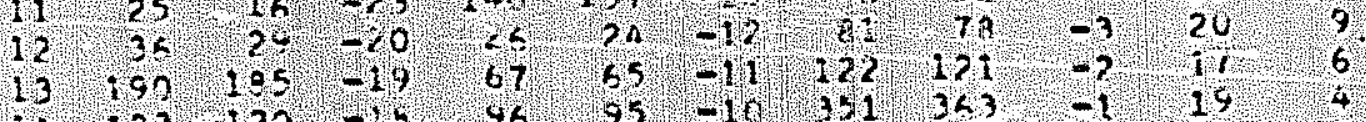

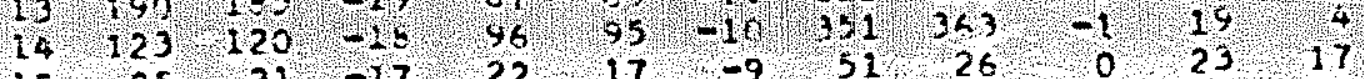

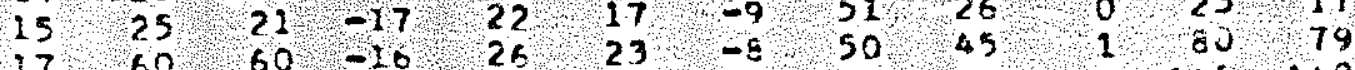

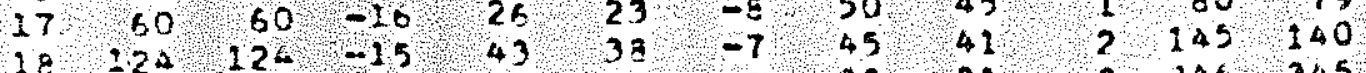

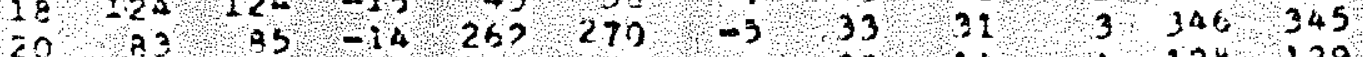

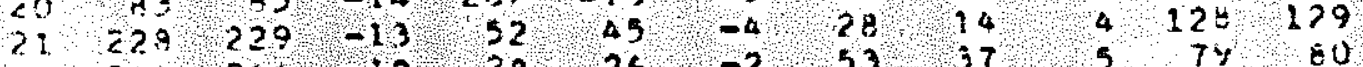

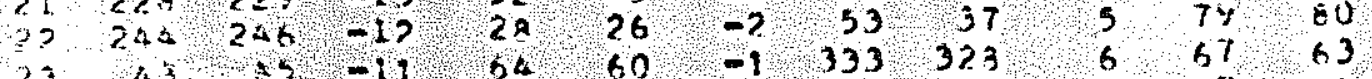

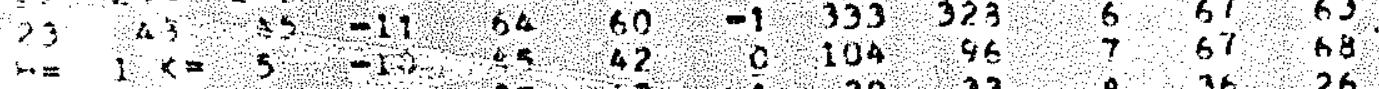

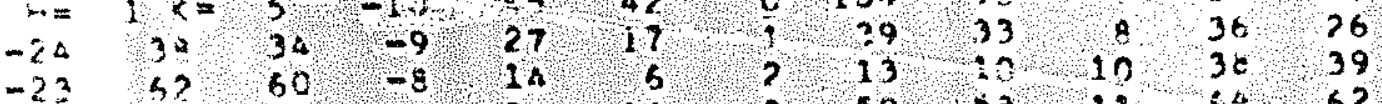

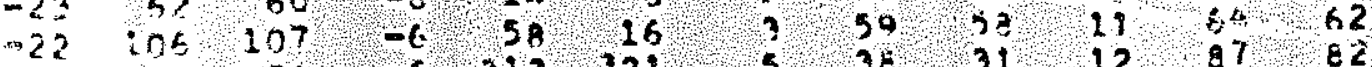

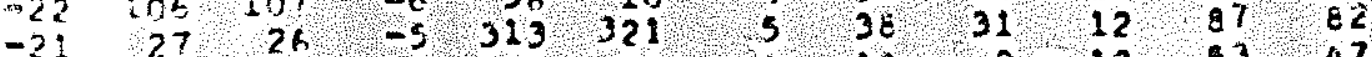

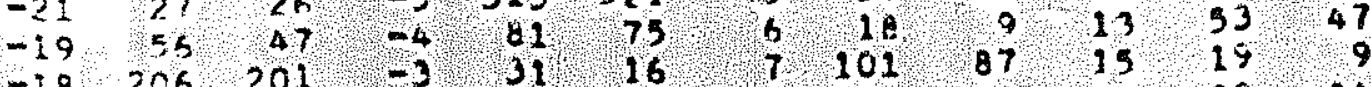

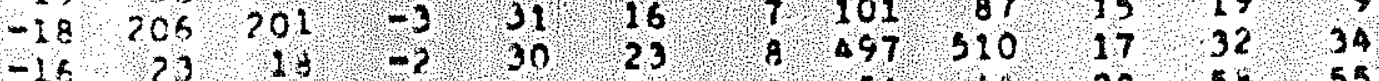

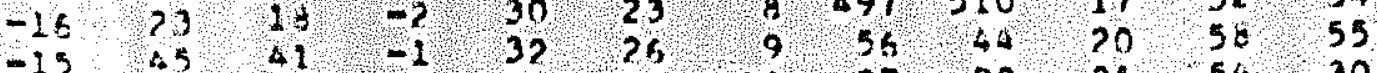

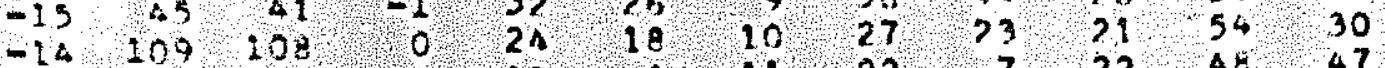

$\begin{array}{llllllllllll}-13 & 35 & 35 & 1 & 15 & 6 & 1 & 22 & 7 & 27 & 46 & 47\end{array}$

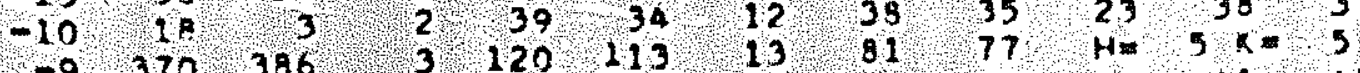

$\begin{array}{lllllllllllll}-9 & 370 & 386 & 3 & 120 & 113 & 13 & 81 & 77 \\ -8 & 156 & 155 & 4 & 265 & 258 & 14 & 67 & 60 & -27 & 41\end{array}$

$\begin{array}{llllllllllll}-7 & 95 & 93 & 5 & 4 a & 43 & 15 & 48 & 40 & -75 & 34 & 30\end{array}$

\begin{tabular}{llllllllllll}
-6 & 5 & 50 & 5 & 6 & 35 & 36 & 16 & 92 & 19 & -24 & 39 \\
\hline
\end{tabular}

$\begin{array}{lllllllllllllllll}-5 & 52 & 51 & 8 & 117 & 119 & 17 & 5 & 26 & -19 & 246 & 257\end{array}$

$\begin{array}{lllllllllllllll}-3 & 10 & 0 & 101 & 10 & 8 & 34 & 39 & 21 & 35 & -14 & 143 & 137\end{array}$

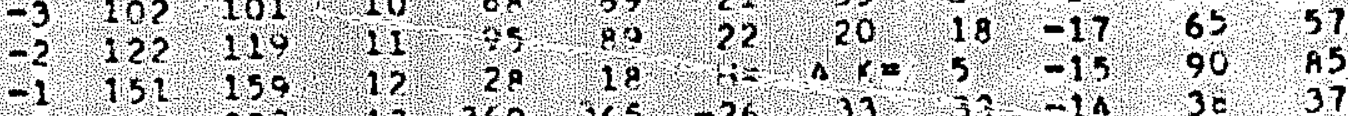

$\begin{array}{lllllllllllllll}0 & 29 ? & 302 & 13 & 360 & 565 & -26 & 33 & 32 & -11 & 36 & 37\end{array}$

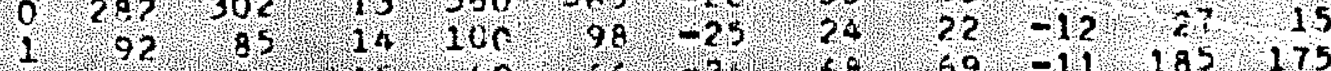

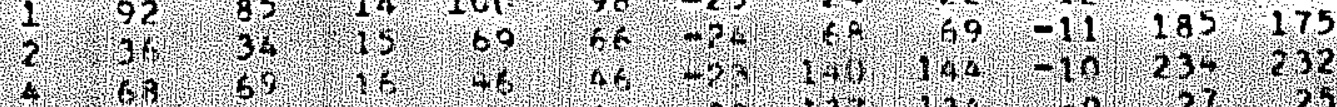

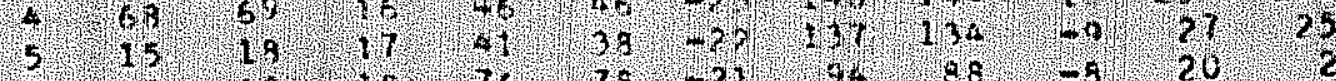

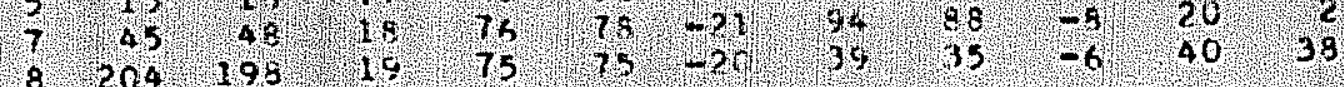




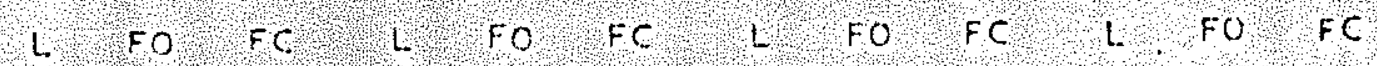

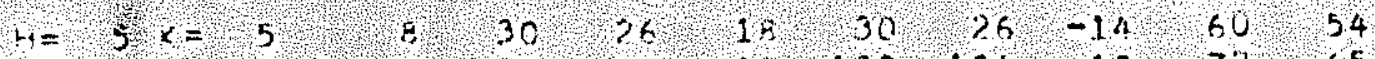

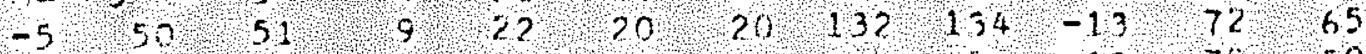

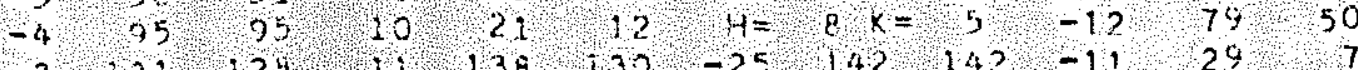

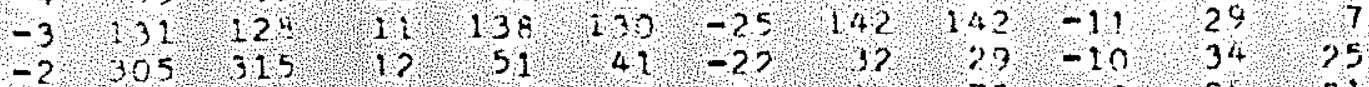

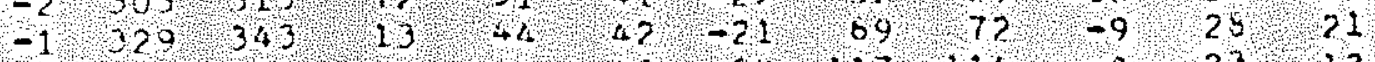

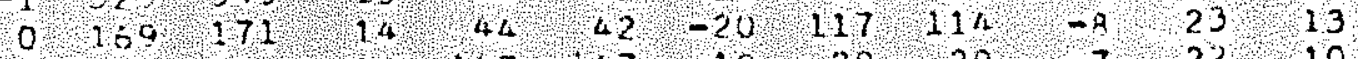

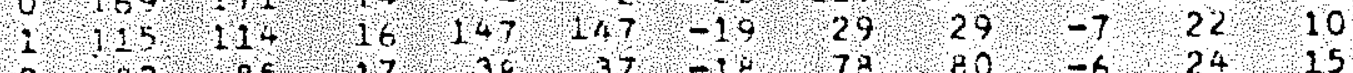

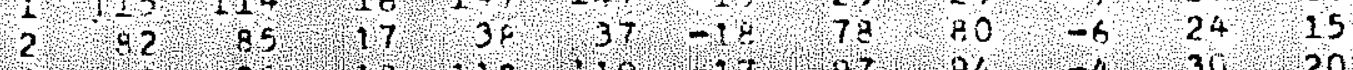

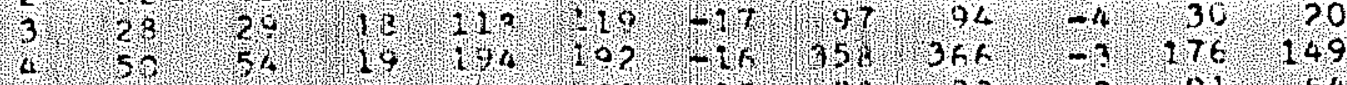

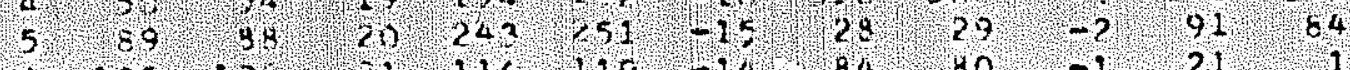

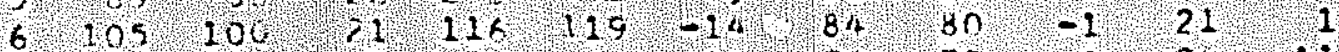

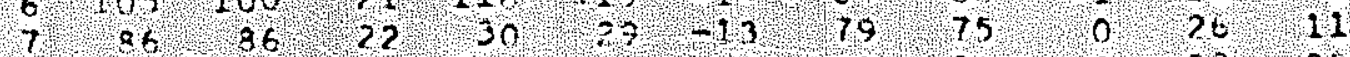

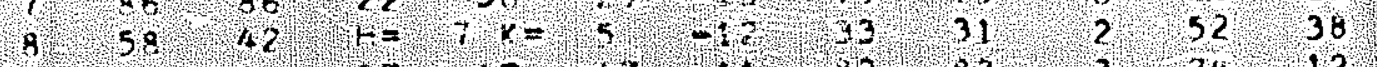

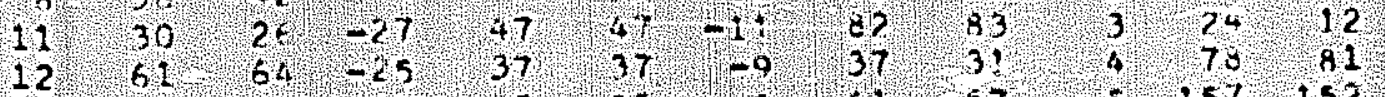

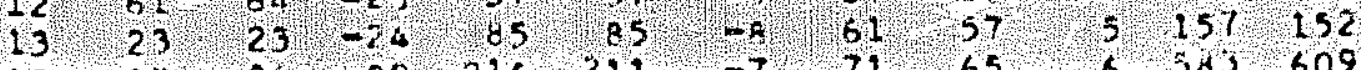

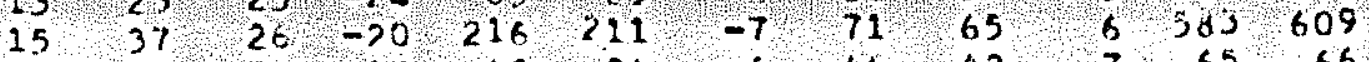

$\begin{array}{lllllllllllll}16 & 90 & 96 & -17 & 43 & 34 & -6 & 46 & 42 & 7 & 65 & 66\end{array}$

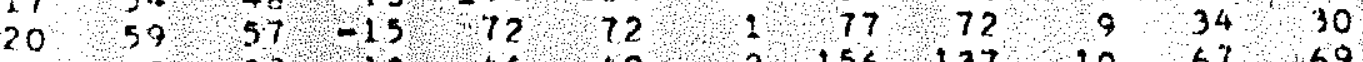

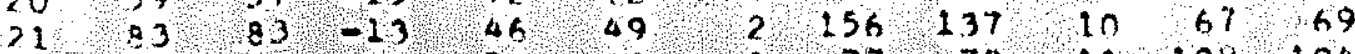

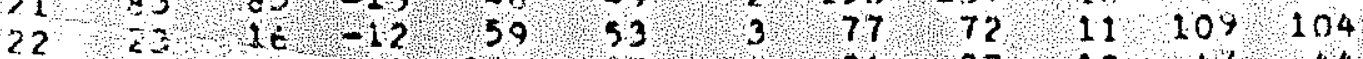

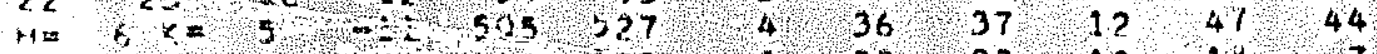

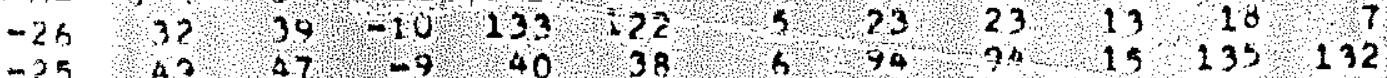

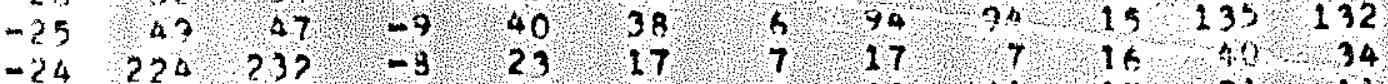

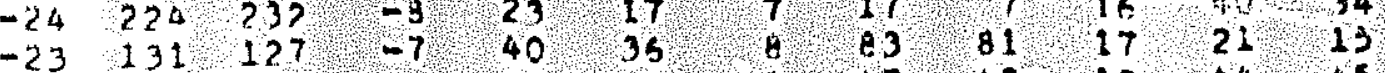

$\begin{array}{llllllllllll}-22 & 30 & 26 & -6 & 35 & 62 & 6 & 19 & 44 & 45\end{array}$

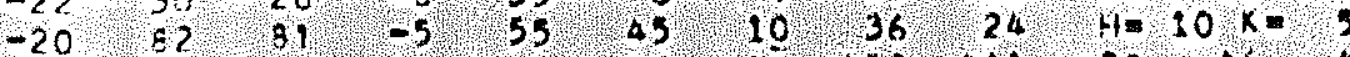

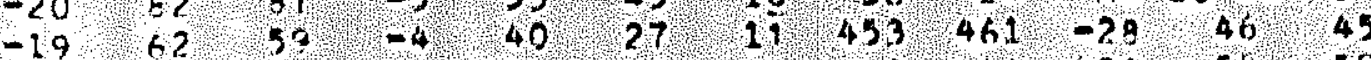

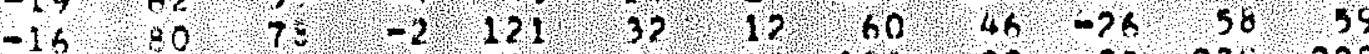

$\begin{array}{lllllllllllll}-15 & 207 & 204 & -1 & 39 & 25 & 15 & 100 & 99 & -25 & 3.30 & 229\end{array}$

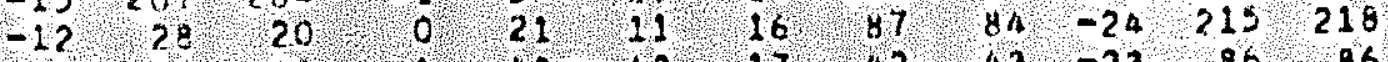

$\begin{array}{lllllllllllll}-11 & 93 & 93 & 1 & 4 A & 43 & 17 & 42 & 43 & -23 & 86 & 86\end{array}$

$\begin{array}{llllllllllllllll}-10 & 65 & 61 & 2 & 38 & 37 & 19 & 28 & 18 & -2 ? & 25 & 22\end{array}$

$\begin{array}{lllllllllllllll}-9 & 65 & 55 & 3 & 20 & 6 & 2 n & 103 & 101 & -21 & 77 & 76\end{array}$

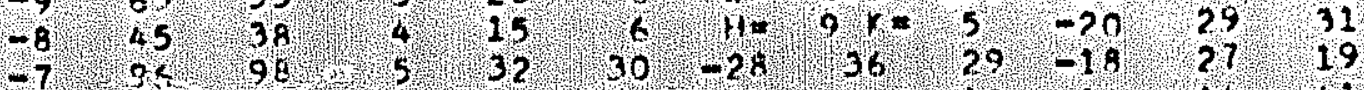

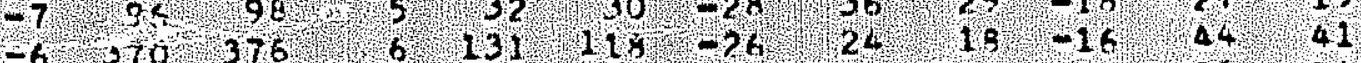

$\begin{array}{llllllllllllll}-5 & 182 & 180 & 7 & 95 & 76 & -25 & 132 & 132 & -15 & 55 & 54\end{array}$

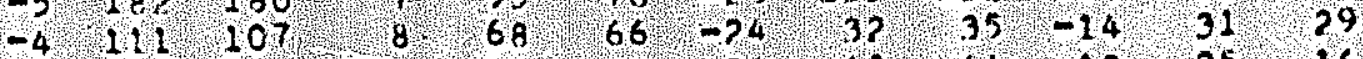

$\begin{array}{lllllllllllll}-3 & 70 & 7 ? & 9 & 5 & 53 & -23 & 63 & 64 & -13 & 25 & 16\end{array}$

$\begin{array}{lllllllllllll}-2 & 60 & 56 & 10 & 40 & 4 ? & -7 ? & 6 ? & 6 ? & -9 & 13\end{array}$

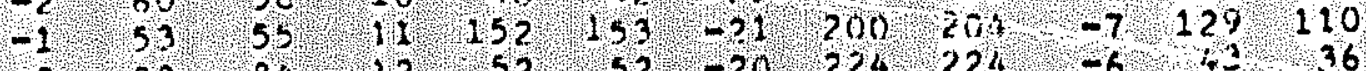

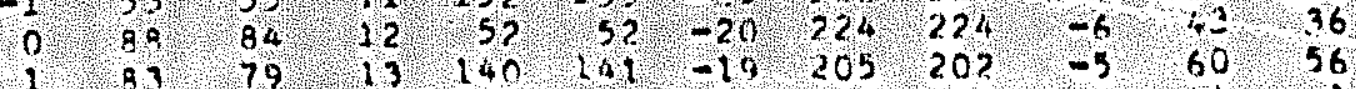

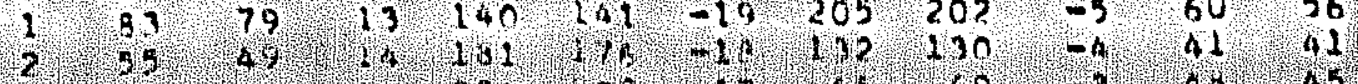

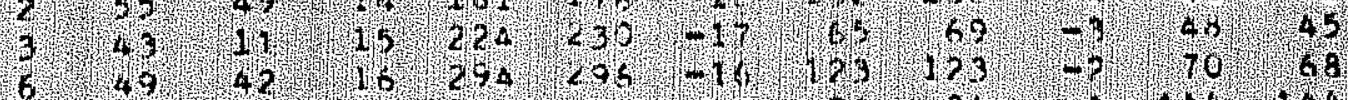

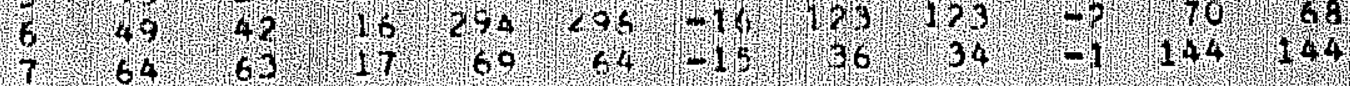




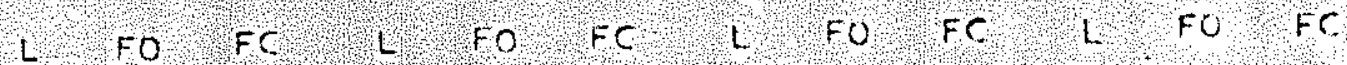

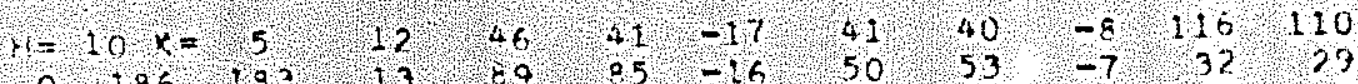

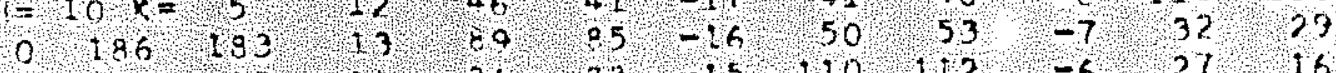

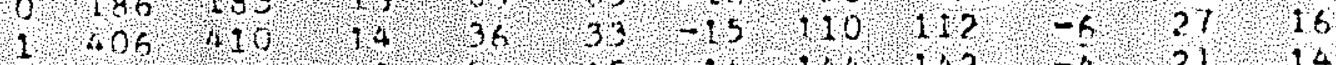

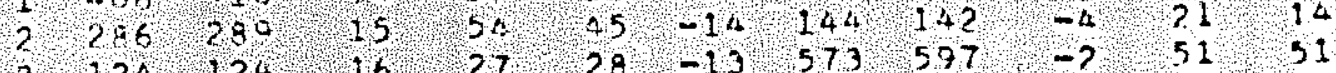

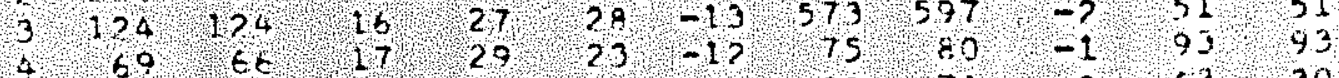

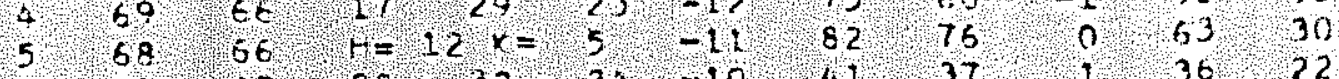

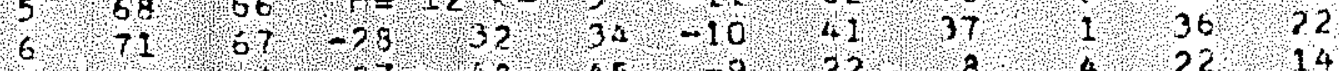

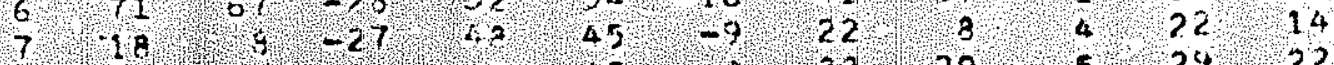

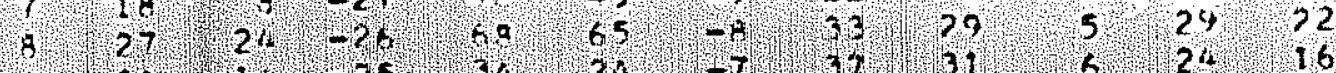

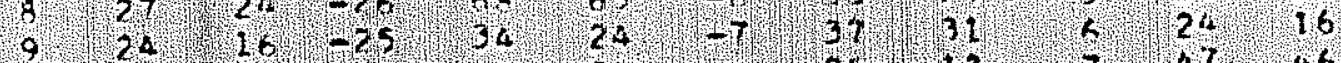

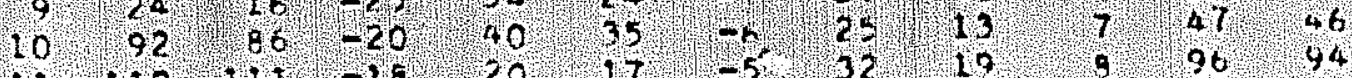

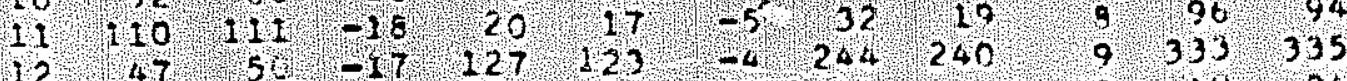

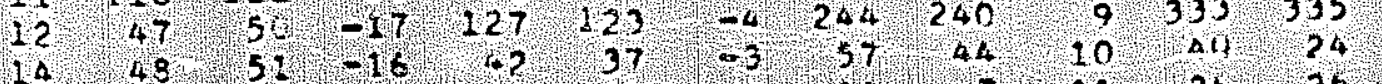

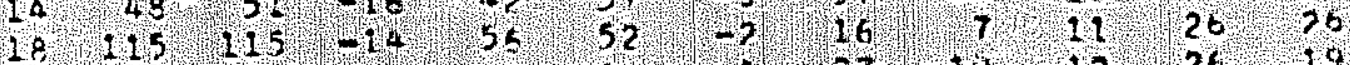

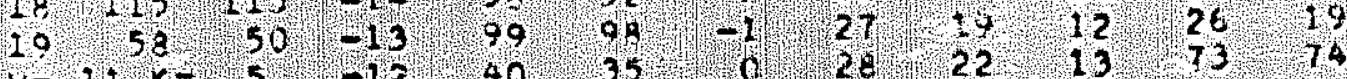

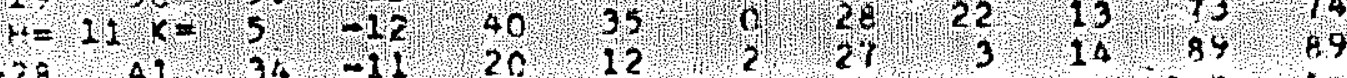

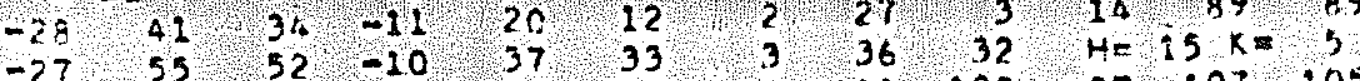

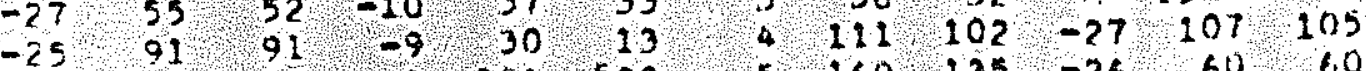

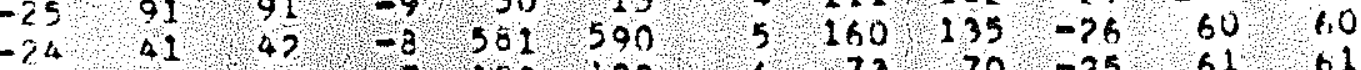

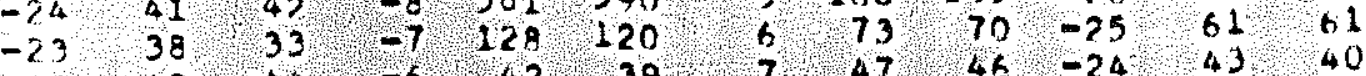

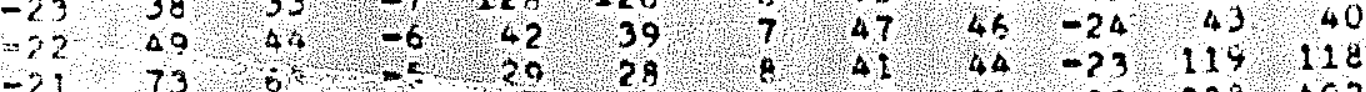

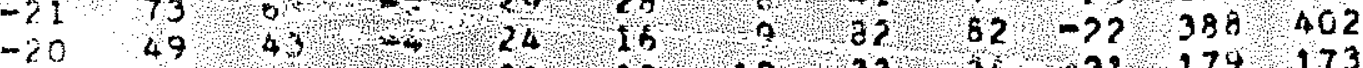

$\begin{array}{llllllllllll}-19 & 12 & 4 & -3 & 29 & 19 & 10 & 33 & 36 & 21 & 179 & 173\end{array}$

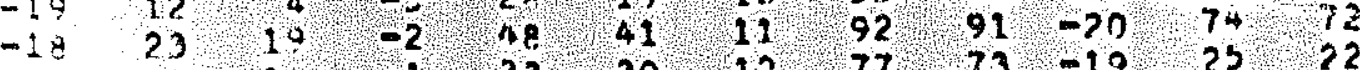

\begin{tabular}{llllllllllll}
-16 & 20 & 17 & -1 & 33 & 30 & 13 & 77 & 73 & -19 & 23 & 12 \\
\hline & 0 & 47 & 34 & 13 & 56 & 53 & -18 & 116 & 114
\end{tabular}

$\begin{array}{lllllllllll}-14 & 26 & 15 & 0 & 47 & 34 & 13 & 56 & 53 & -18 & 116 \\ -13 & 85 & 76 & 2 & 211 & 205 & 14 & 256 & 262 & -17 & 33\end{array}$

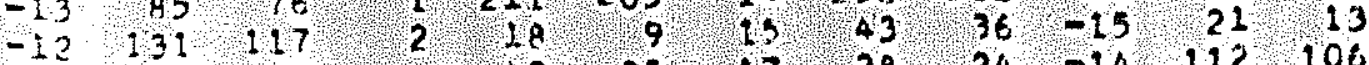

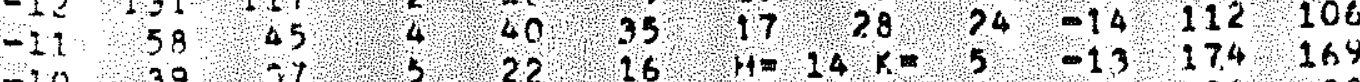

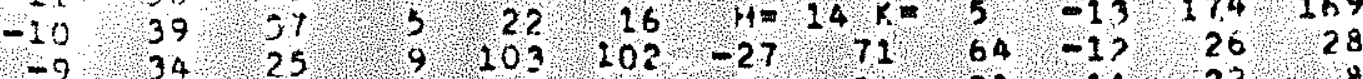

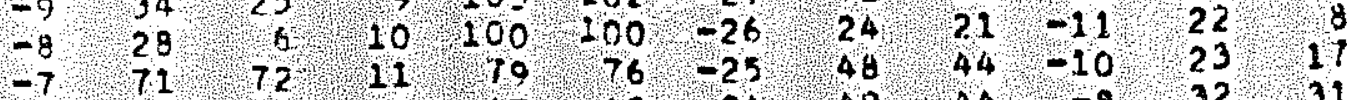

$\begin{array}{lllllllllllll}-6 & 109 & 106 & 12 & 67 & 65 & -24 & 49 & 44 & -8 & 32 & 31\end{array}$

$\begin{array}{lllllllllllllll}-5 & 114 & 11 ! & 13 & 61 & 59 & -23 & 46 & 41 & -7 & 57 & 56\end{array}$

$\begin{array}{llllllllllll}-4 & 238 & 245 & 14 & 110 & 11 & -27 & 103 & 100 & -5 & 56 & 36\end{array}$

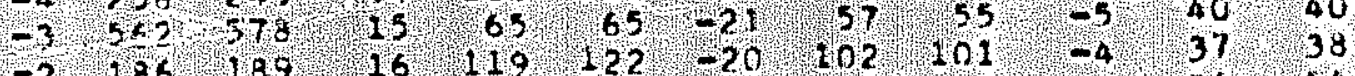

$\begin{array}{llllllllllllll}-2 & 186 & 189 & 16 & 119 & 122 & -20 & 112 & 11 & 3 & -3 & 56 & 54\end{array}$

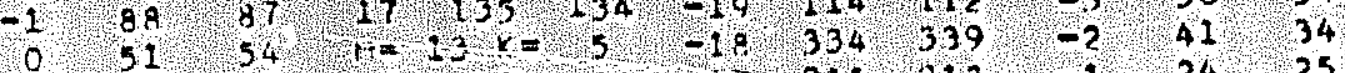

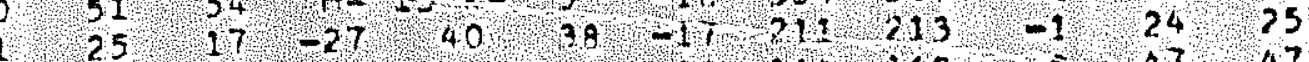

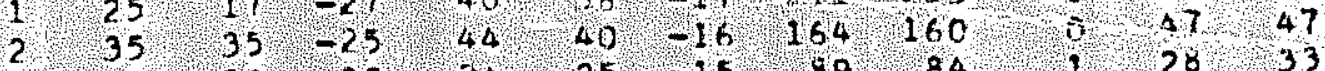

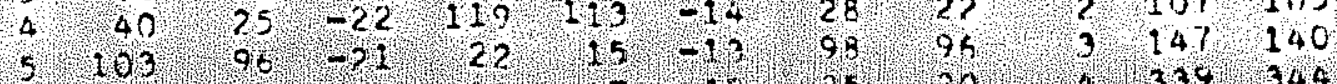

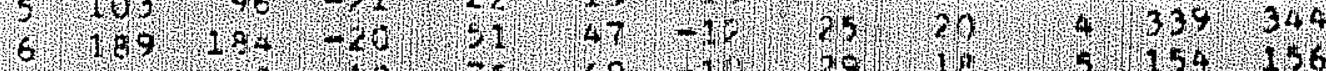

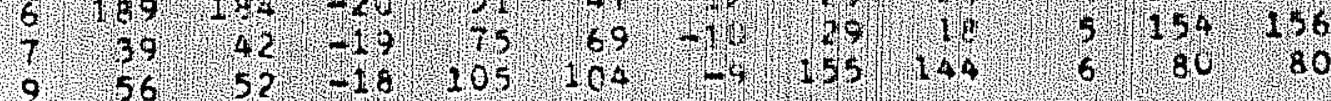




\begin{tabular}{|c|c|c|c|c|c|c|c|c|c|c|c|}
\hline$y=$ & $15 x=$ & 5 & -14 & 119 & 107 & 3 & 57 & 54 & -14 & $5 \%$ & \\
\hline$?$ & 40 & 32 & -13 & 100 & 97 & 3 & 28 & & -13 & 36 & \\
\hline 8 & 38 & 37 & $-1 ?$ & 30 & 16 & 5 & 31 & 36 & -11 & 199 & \\
\hline 3 & 107 & 108 & -11 & 36 & 34 & & 25 & & -10 & 222 & \\
\hline 10 & 21 & & -10 & 27 & 13 & & 40 & $5 \%$ & -9 & 41 & \\
\hline 13 & 169 & 16 & -9 & 50 & 4 & & 309 & & -8 & 27 & \\
\hline 1 & 83 & & -8 & 67 & 6 & 9 & 87 & & -5 & 47 & \\
\hline 15 & 33 & & -7 & 31 & 24 & 10 & 49 & & -4 & 105 & \\
\hline$M=$ & $16 . k=$ & 5 & -6 & 87 & 48 & 11 & 158 & & -3 & 131 & \\
\hline-27 & 310 & $\%$ & -5 & 163 & 441 & $H=$ & $10, k=$ & 5 & -2 & 240 & \\
\hline & 128 & 12 & -4 & 80 & 78 & -28 & 77 & & -1 & 154 & \\
\hline 4 & 23 & 1 & -3 & 12 & 証 & -24 & 17 & & 0 & 173 & \\
\hline & 75 & & -1 & 41 & 3,3 & -23 & 67 & & 1 & 41 & \\
\hline 2 & 93 & 9 & 0 & 32 & 33 & $=22$ & $1 \%$ & 17 & 2 & 42 & \\
\hline 19 & 66 & 62 & 1 & 21 & 26 & .21 & 107 & & 3 & 50 & \\
\hline 8 & 122 & 11 & 3 & 118 & 108 & -20 & 111 & & 6 & 60 & \\
\hline 4 & 21 & XI & 4 & 270 & 258 & -19 & 42 & 48 & 7 & 129 & \\
\hline 14 & 40 & 3 & 5 & 27 & 75 & -18 & 86 & 86 & $\mathrm{H}=$ & $21 \mathrm{r}=$ & \\
\hline 13 & 36 & 3 & 7 & 38 & 28 & -17 & 130 & & -26 & 10 & \\
\hline 12 & 39 & 12 & 8 & 85 & 83 & -16 & 136 & & -74 & 205 & \\
\hline 11 & 25 & 1 & 10 & $6 t$ & 63 & -15 & 342 & & $=3$ & 81 & \\
\hline 10 & 30 & 2 & 11 & 107 & 992 & -14 & 148 & $1 \%$ & -21 & 30 & \\
\hline$-r$ & 37 & 2 & 12 & 126 & 133 & -13 & 9 & & -20 & 10 & \\
\hline & 73 & 8 & 13 & $19 ?$ & 196 & -12 & 36 & & -19 & 73 & \\
\hline- & 63 & 60 & 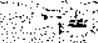 & 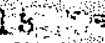 & 4 & -11 & 23 & & -17 & 25 & \\
\hline-6 & 51 & 5 & -19 & 36 & 30 & -9 & 36 & & -14 & 111 & \\
\hline-5 & 40 & 4 & $->9$ & 5 & 5. & -4 & 29 & $?$ & -15 & 275 & \\
\hline-1 & 55 & $\longdiv { 5 }$ & $=27$ & 3 & 28 & -6 & 311 & & -14 & 34 & \\
\hline$=$ & 116 & 11 & -26 & 22 & 14 & -5 & 141 & 13 & -13 & 32 & \\
\hline-7 & 109 & 10 & -23 & 8 & 82 & -4 & 47 & 4 & -10 & 64 & \\
\hline-1 & 189 & 13 & -21 & 2 & 19 & -2 & 43 & 4 & -9 & 85 & \\
\hline 0 & 300 & & -20 & 3 & 2 & 1 & 70 & & -9 & 72 & \\
\hline 1 & 99 & 9 & -10 & 5 & 4 & ? & 13 & 11 & -7 & $1 / 5$ & \\
\hline 2 & 36 & 3 & -18 & 6 & 6 & 3 & 397 & 40 & -6 & 34 & \\
\hline 3 & 19 & 1 & -17 & 4 & 4 & 6 & & & -3 & 143 & \\
\hline 4 & 880 & $\$ 7$ & -16 & 5 & 5 & 8 & & & -4 & $6^{4}$ & \\
\hline 8 & 149 & 13 & -15 & 75 & 7 & 9 & 68 & & -3 & 58. & \\
\hline 09 & 167 & 16 & -14 & 1 & 18 & 10 & 24 & 1 & -2 & $2 \%$ & \\
\hline 10 & 133 & 3 & -13 & 3 & 28 & $H=$ & $20 \mathrm{k}=$ & 5 & -1 & 34 & \\
\hline 12 & 34 & 3 & -12 & 85 & 8 & -24 & 69 & 5 & $n$ & & \\
\hline 13 & 00 & 3 & -11 & 9 & 87 & -27 & 131 & 11 & 1 & 81 & \\
\hline $17=$ & $17 \mathrm{x}=$ & 5 & -10 & 417 & 419 & -76 & 72 & 7 & 2 & 91 & \\
\hline 29 & 473 & 7 & -9 & 38 & 4 & $->5$ & 46 & n & 3 & 70 & \\
\hline 7 & 139 & 14 & -8 & 31 & 19 & 23 & 48 & & 6 & 21 & \\
\hline & 43 & 4 & -5 & 2 & 19 & -22 & 78 & 7 & 7 & 80 & \\
\hline & 30 & 2 & -4 & 3 & 29 & -21 & 64 & & $1 /=$ & $22,1=$ & \\
\hline 4 & 49 & 4 & -3 & 4 & 32 & -20 & 158 & 16 & -27 & 52 & \\
\hline 3 & 107 & 10 & -2 & 3, & $2 ?$ & -19 & 2.45 & 23 & -36 & 40 & \\
\hline & 51 & 4 & -1 & 310 & 301 & -18 & 173 & 12 & -25 & 17 & \\
\hline & 49 & 4 & 0 & 47 & 38 & -17 & 46 & & -24 & 89 & \\
\hline & 21 & 2 & 1 & 21 & 27 & -16 & 22 & & 72 & 19 & \\
\hline & 47 & 10 & 2 & 25 & 16 & - 19 & H67 & & 47 & 39 & \\
\hline
\end{tabular}




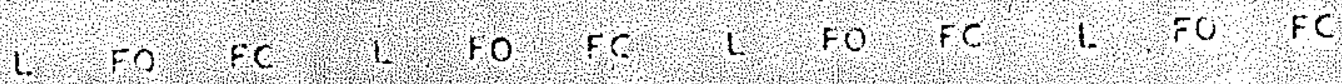

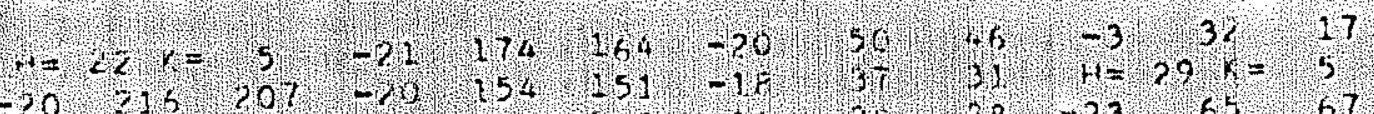

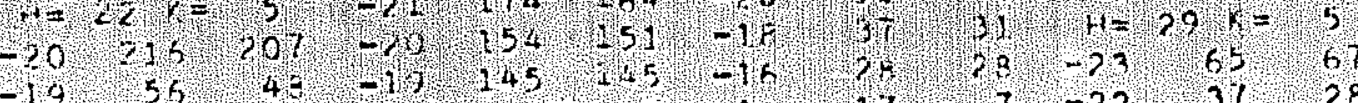

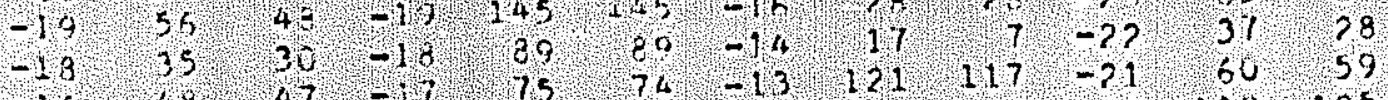

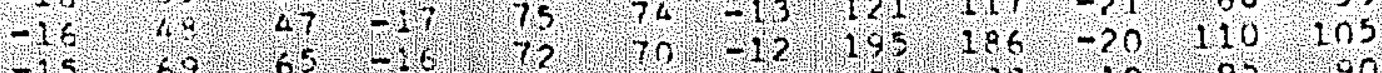

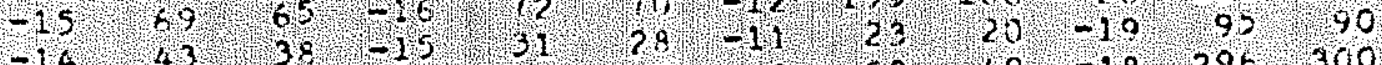

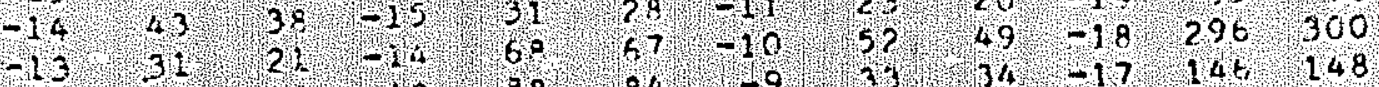

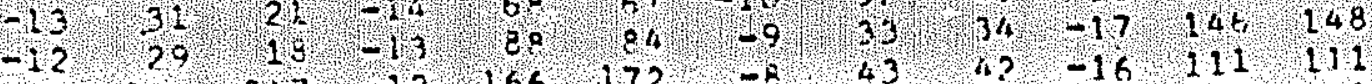

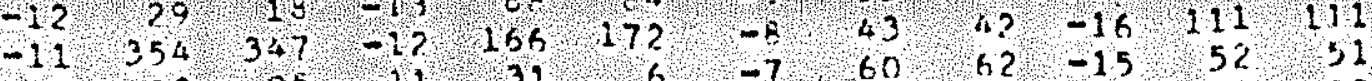

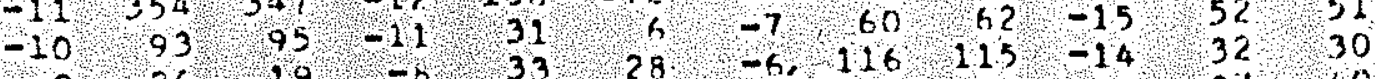

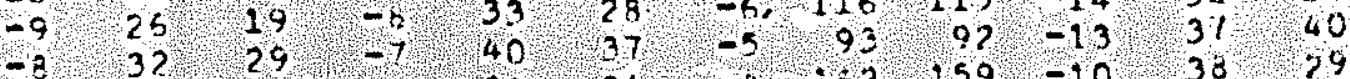

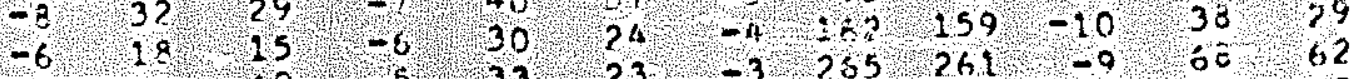

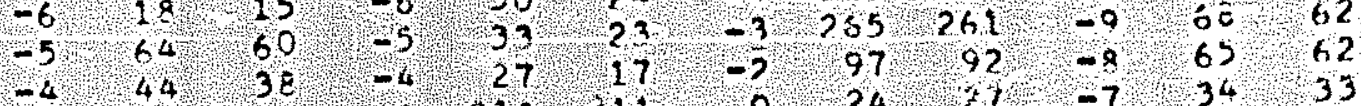

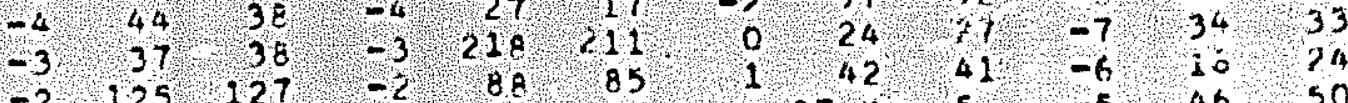

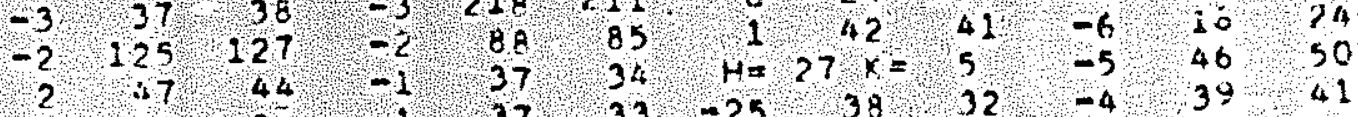

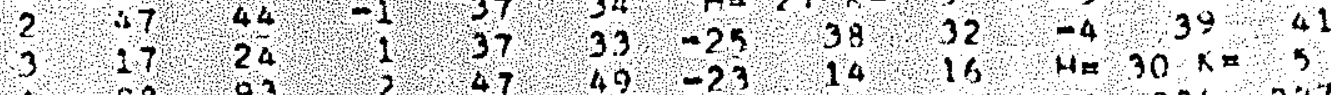

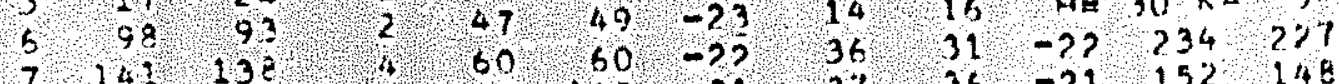

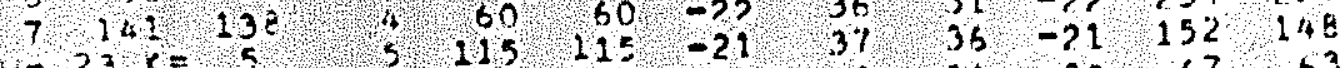

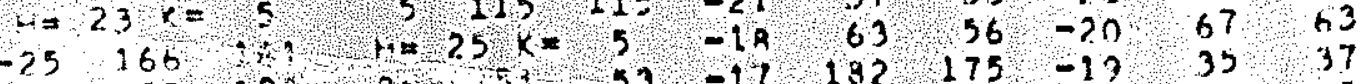

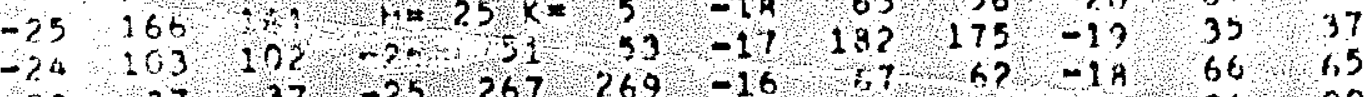

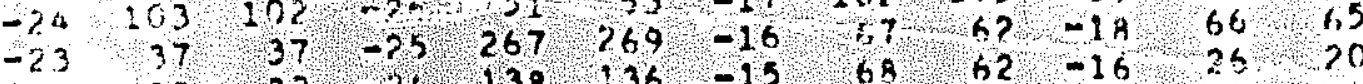

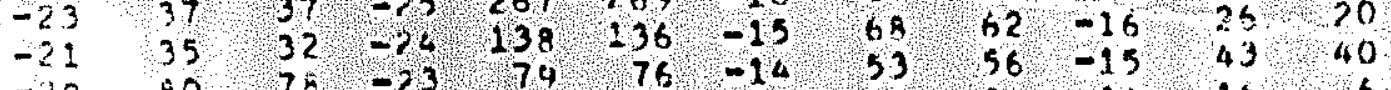

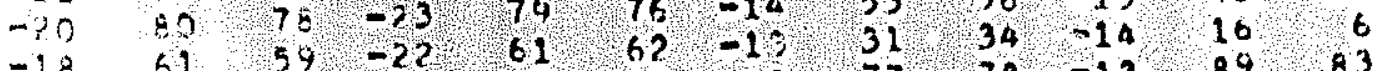

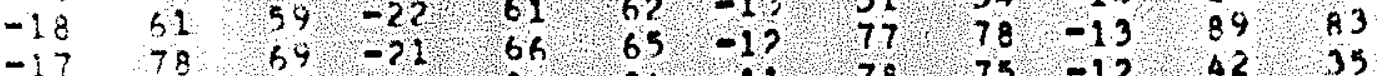

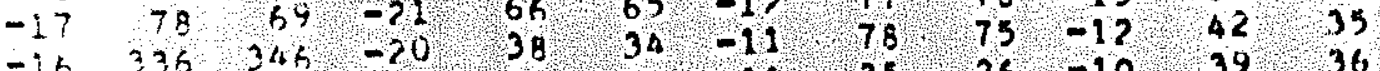

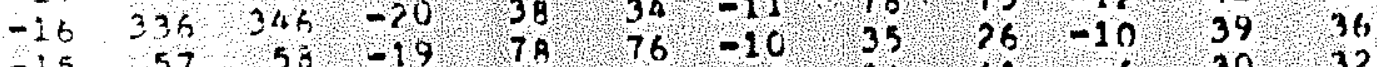

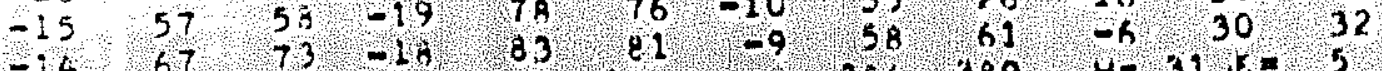

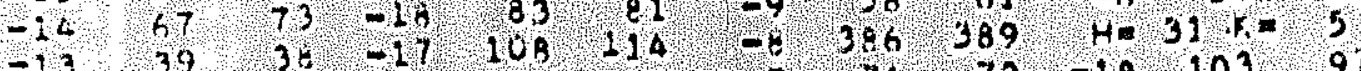

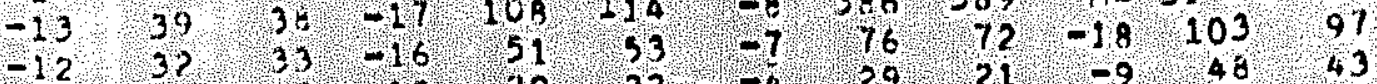

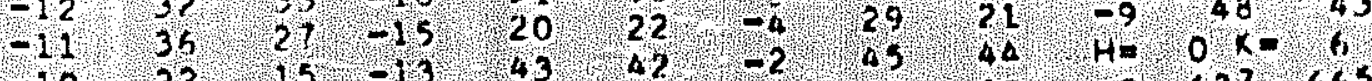

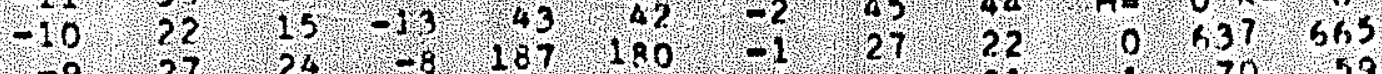

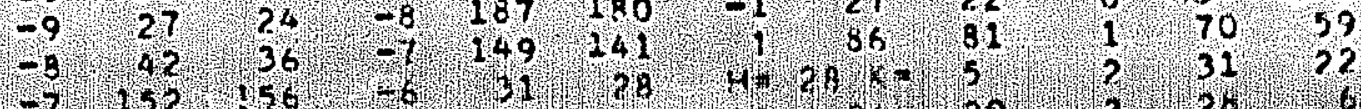

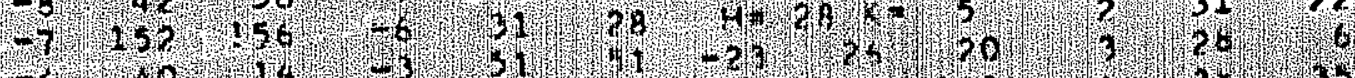

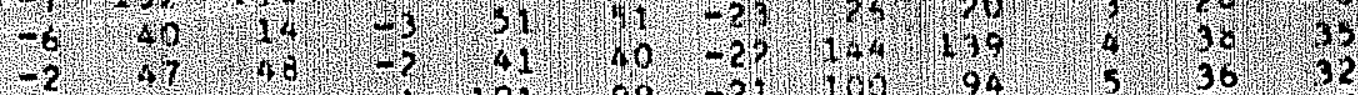

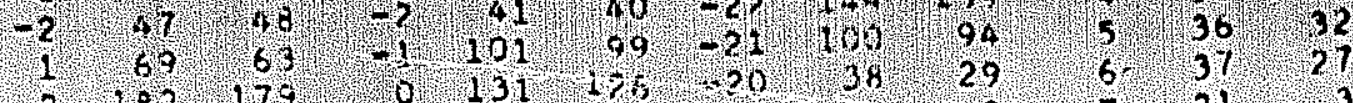

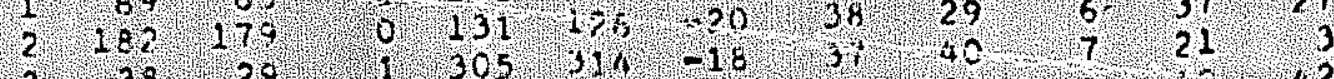

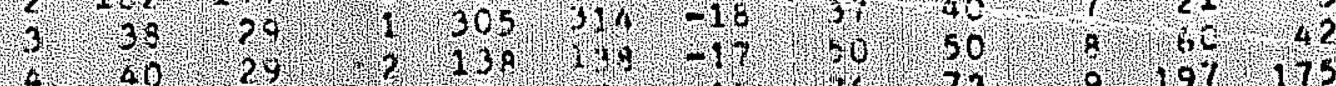

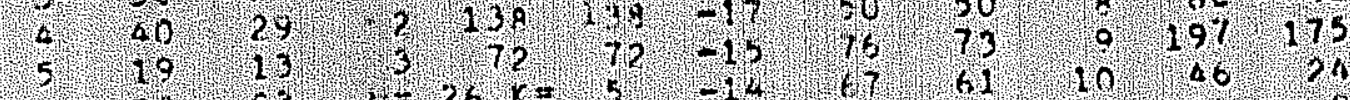

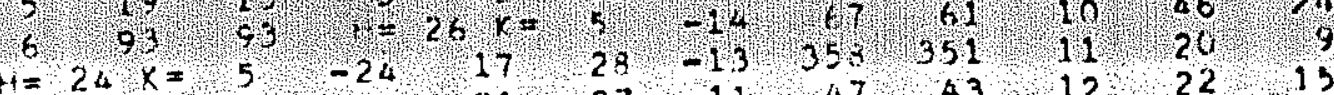

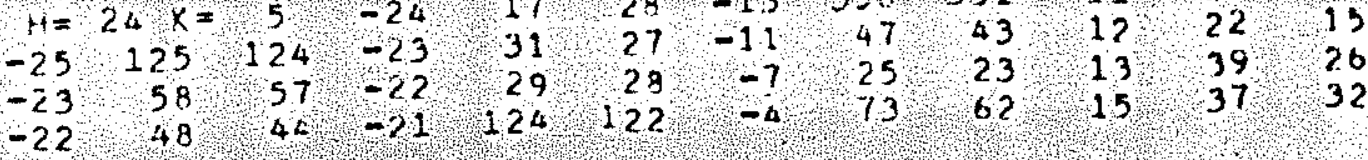




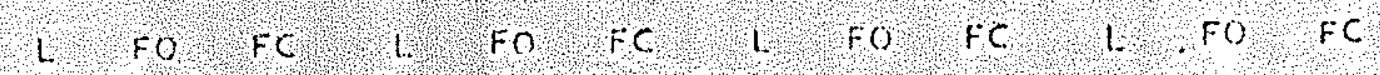

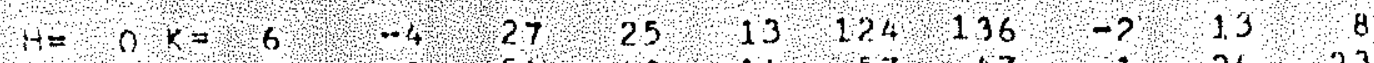

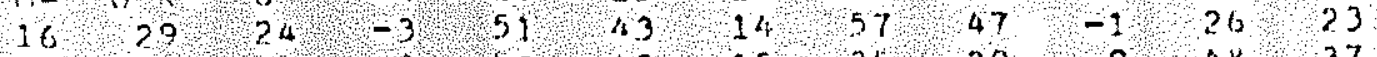

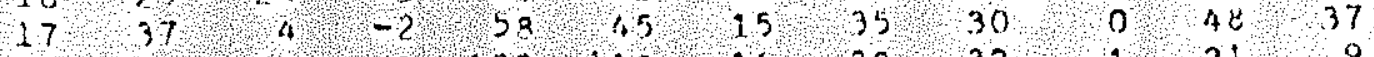

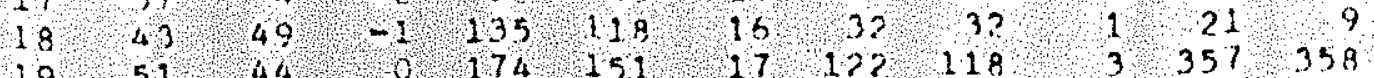

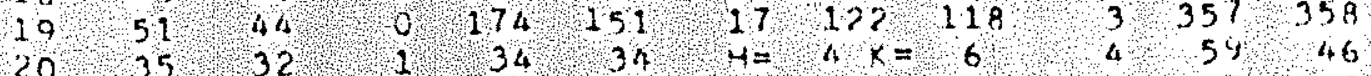

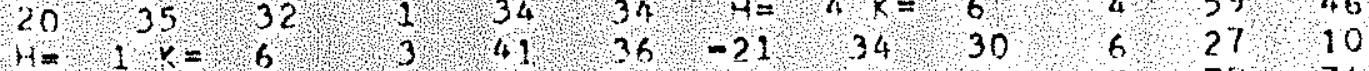

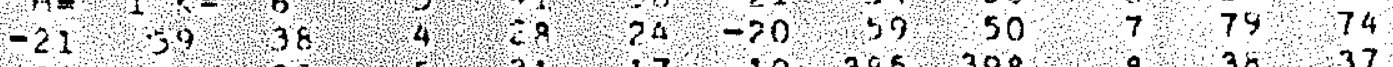

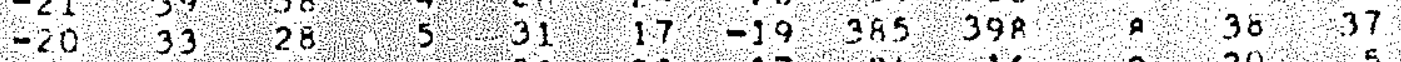

$\begin{array}{llllllllllll}-17 & 31 & 25 & 6 & 30 & 20 & -17 & 24 & 16\end{array}$

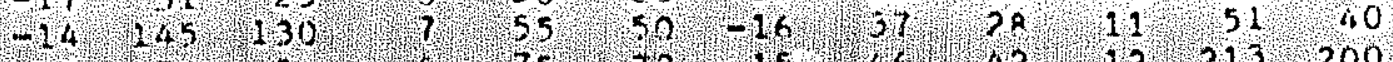

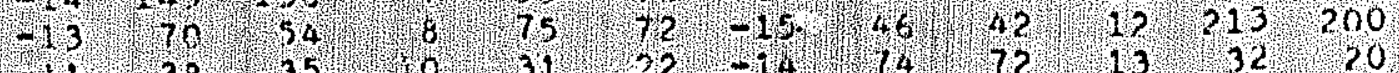

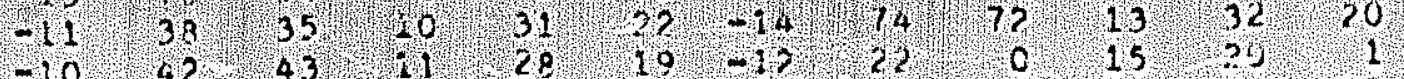

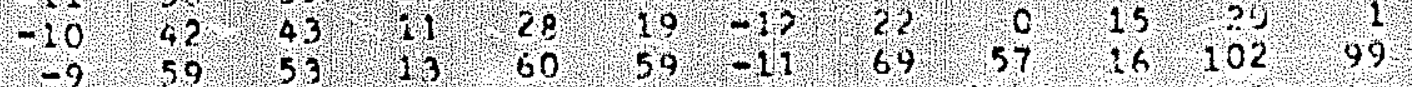

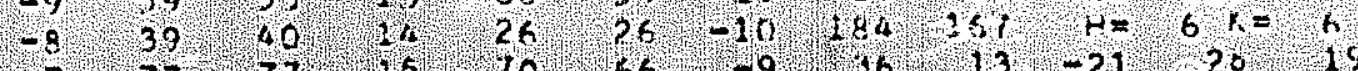

$\begin{array}{lllllllllll}-7 & 77 & 77 & 15 & 70 & 66 & -9 & 96 & 13 & -21 & 28\end{array}$

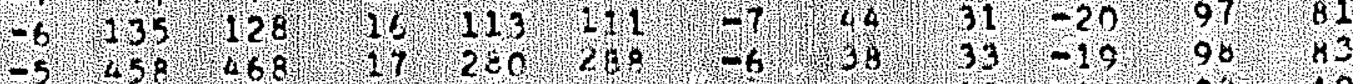

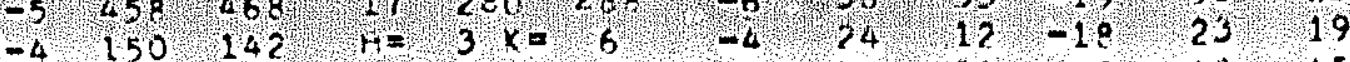

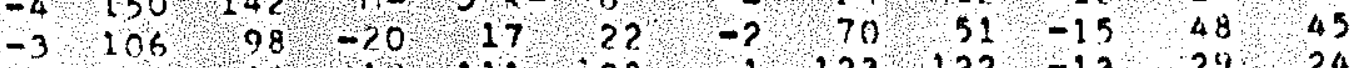

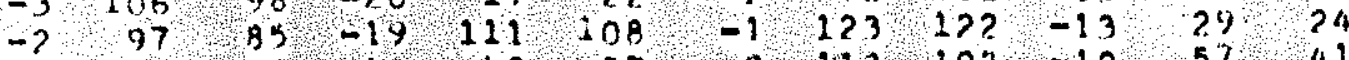

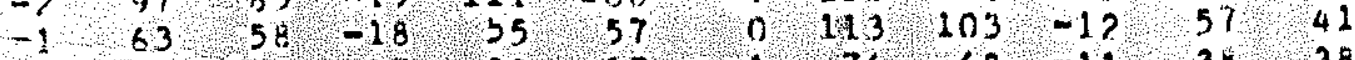

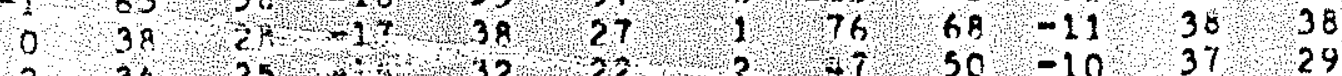

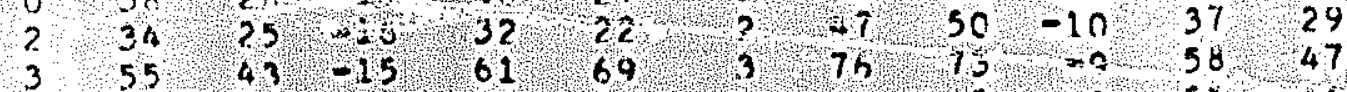

$\begin{array}{llllllllllll}4 & 152 & 136 & -110 & 399 & 409 & 4 & 64 & 65 & -8 & 55 & 40\end{array}$

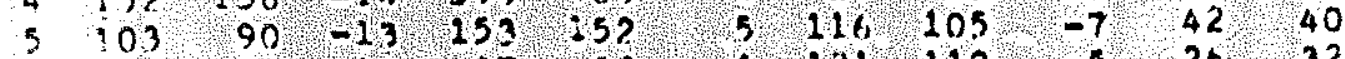

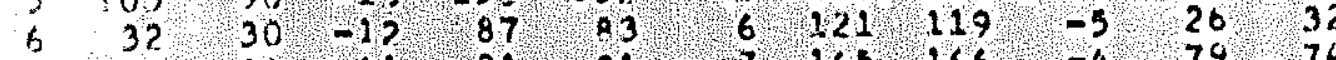

$\begin{array}{cccccccccccc}8 & 39 & 28 & -11 & 31 & 31 & 7 & 169 & 166 & -4 & 79 & 74 \\ 9 & 17 & 9 & -10 & 84 & 8 ? & 8 & 279 & 295 & -3 & 129 & 122\end{array}$

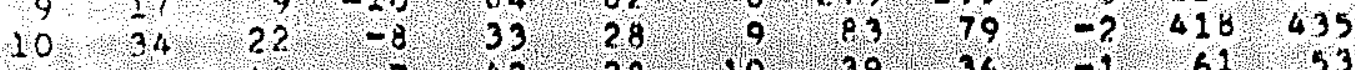

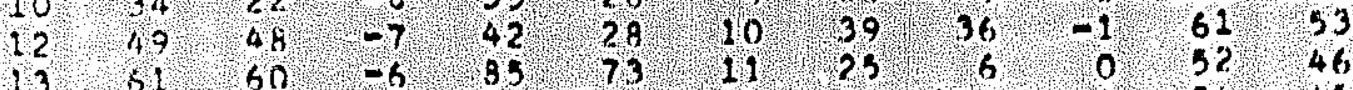

$\begin{array}{llllllllllllll}13 & 14 & 50 & -5 & 85 & 73 & 11 & 23 & 6 & 0 & 53 & 45\end{array}$

$\begin{array}{llllllllllll}15 & 29 & 23 & -4 & 30 & 19 & 15 & 38 & 34 & 2 & 41 & 45\end{array}$

\begin{tabular}{llllllllllllll}
17 & 70 & 72 & -3 & 21 & 13 & 16 & $12 ?$ & 109 & 3 & 39 & 33 \\
\hline
\end{tabular}

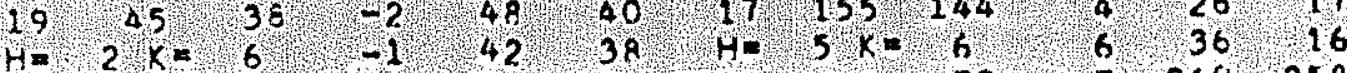

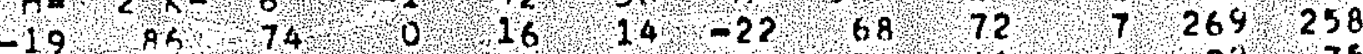

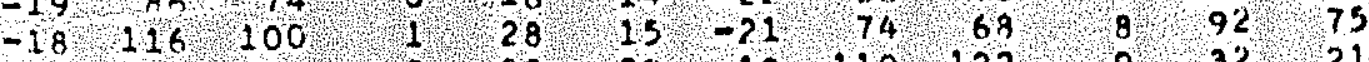

$\begin{array}{lllllllllllll}-16 & 22 & 16 & 2 & 33 & 20 & -19 & 119 & 122 & 9 & 32 & 21\end{array}$

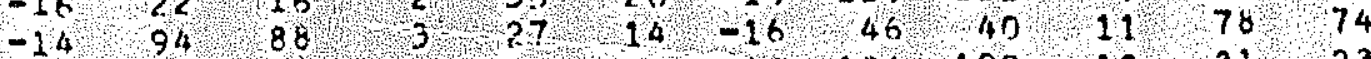

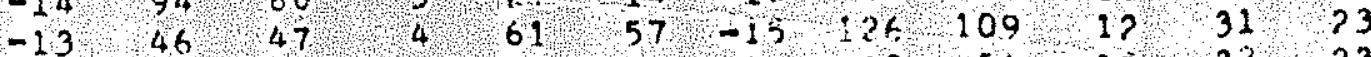

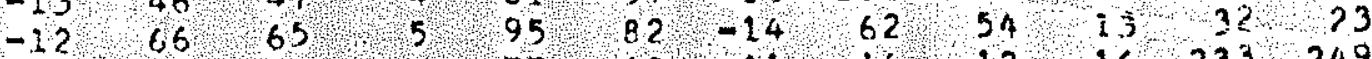

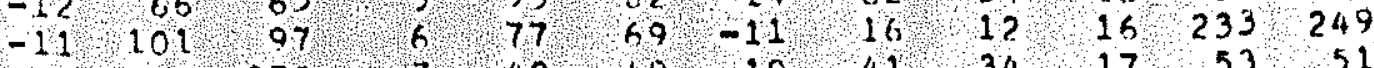

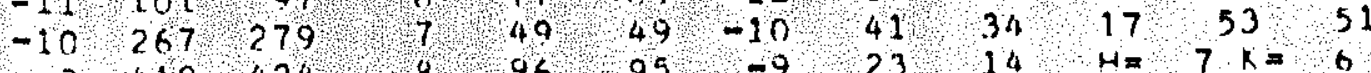

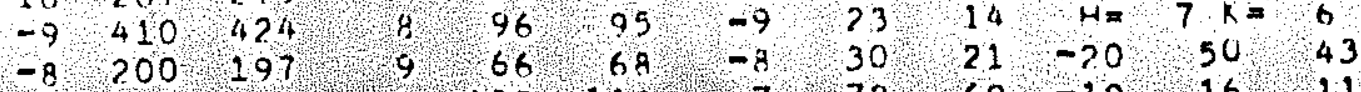

$\begin{array}{lllllllllll}-7 & 139 & 130 & 10 & 125 & 118 & -7 & 72 & 60 & -19\end{array}$

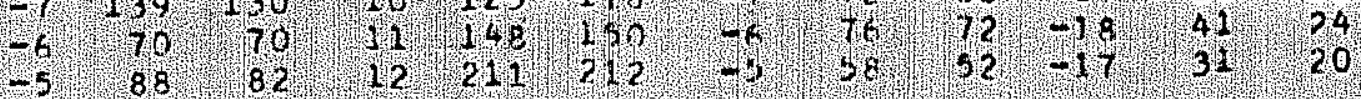




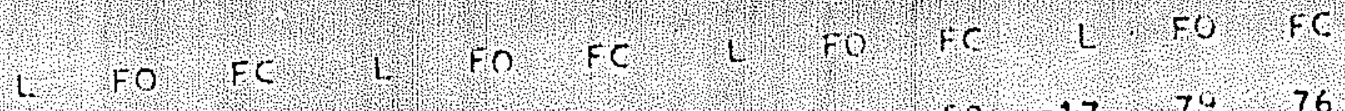

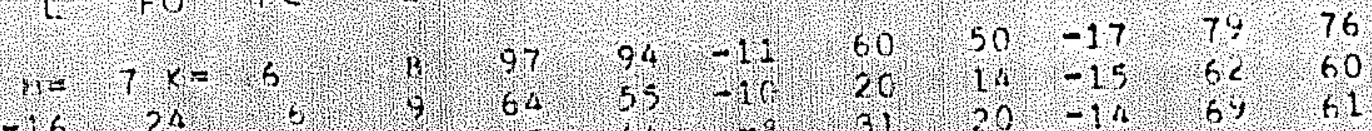

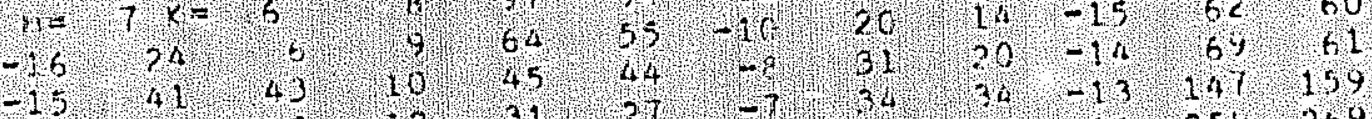

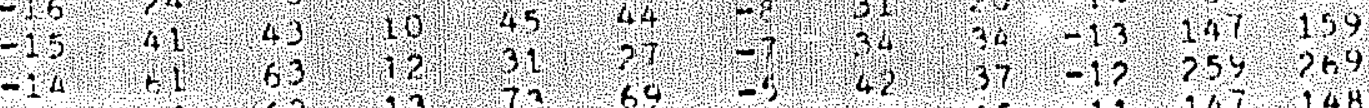

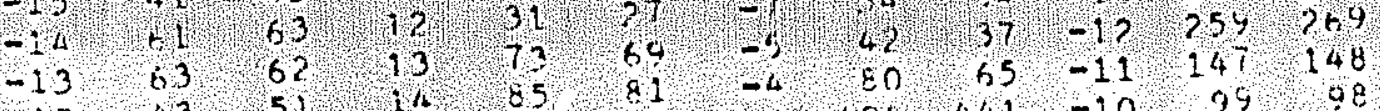

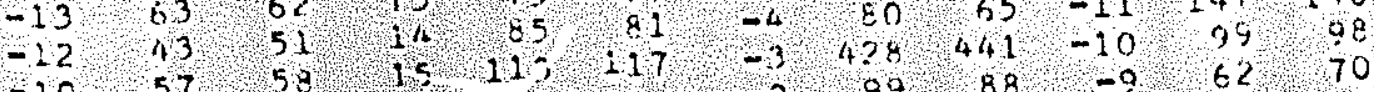

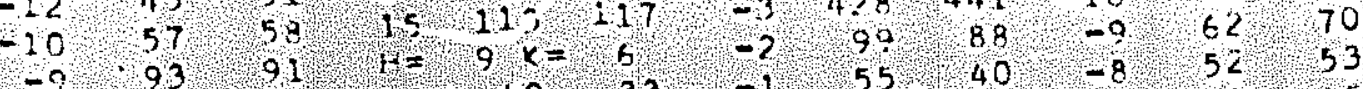

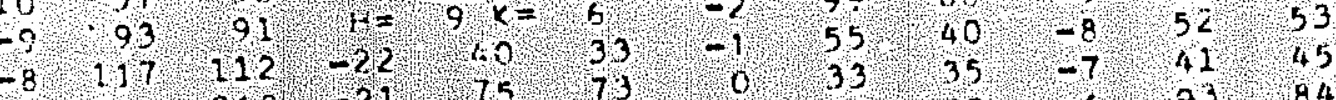

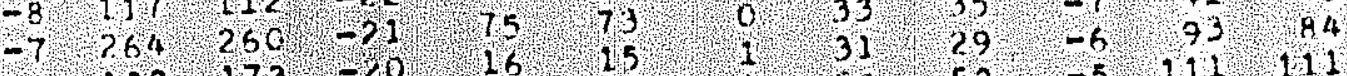

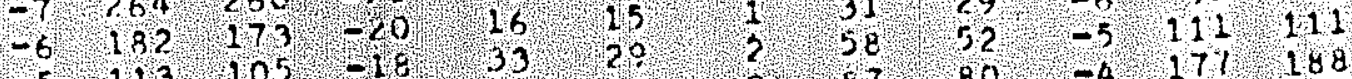

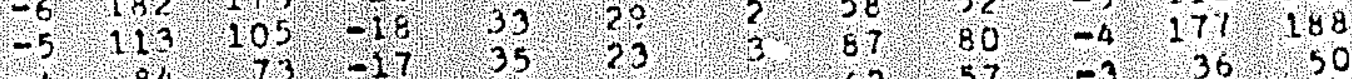

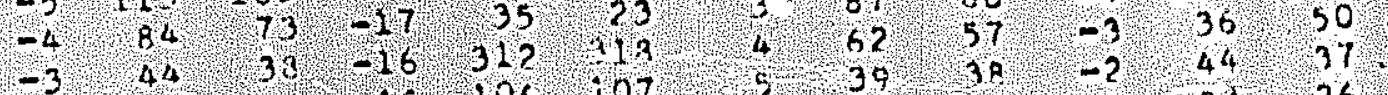

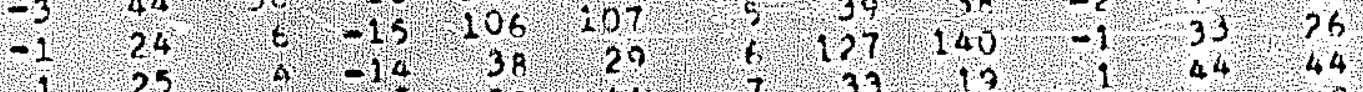

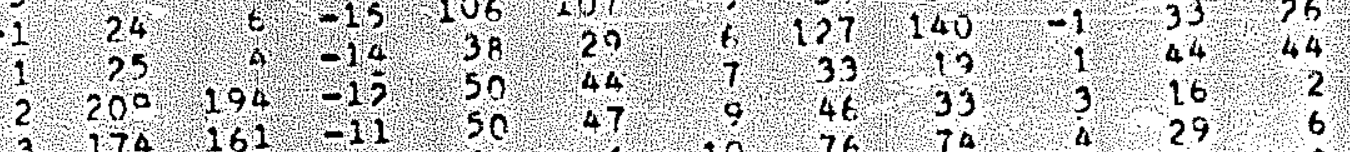

$\begin{array}{lllllllllllll}3 & 174 & 161 & -11 & 50 & 47 & 10 & 10 & 76 & 74 & 4 & 6\end{array}$

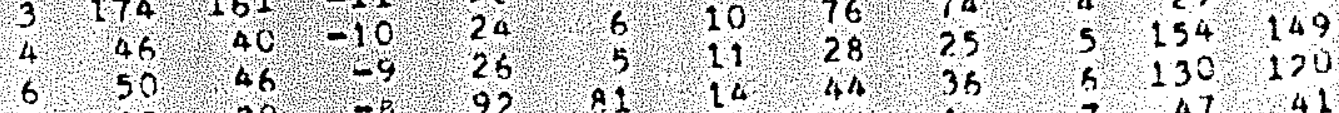

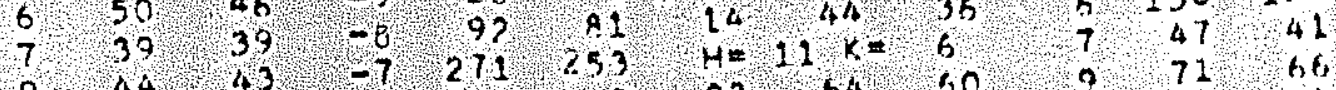

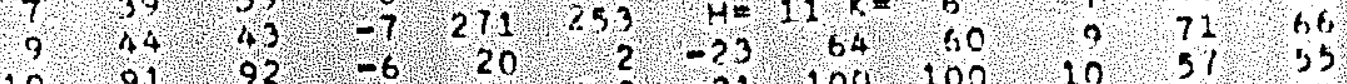

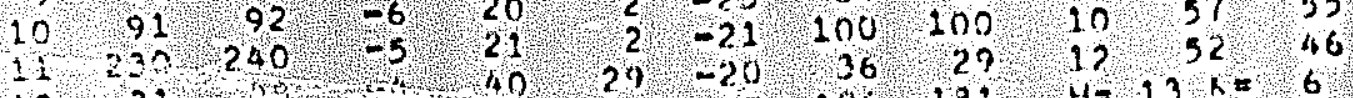

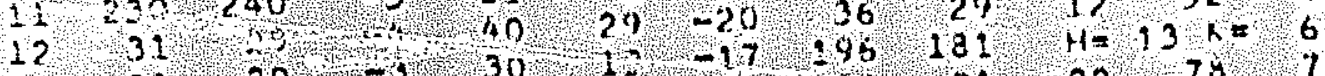

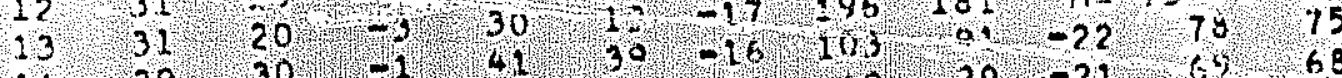

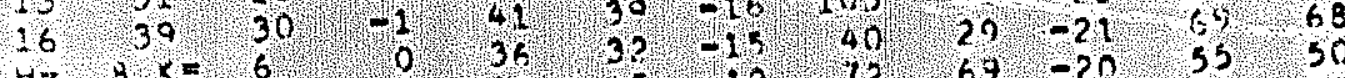

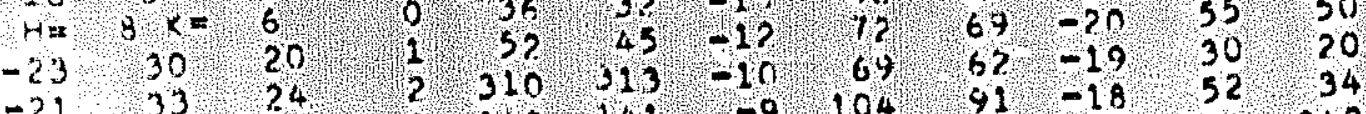

$\begin{array}{lllllllllll}-21 & 133 & 24 & 3 & 310 & 54 & 34\end{array}$

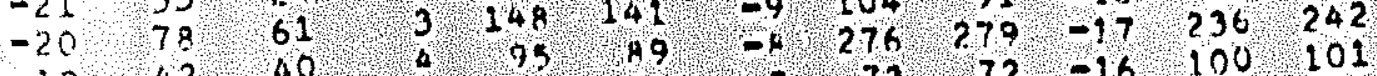

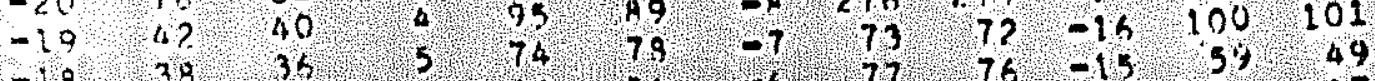

$\begin{array}{lllllllllllllll}-18 & 39 & 36 & 6 & 57 & 54 & -5 & 77 & 76 & -15 & 31 & 17\end{array}$

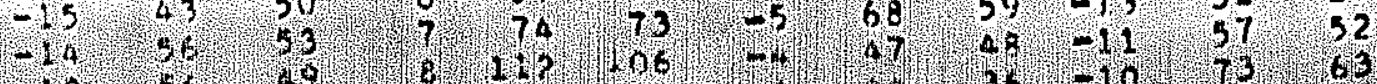

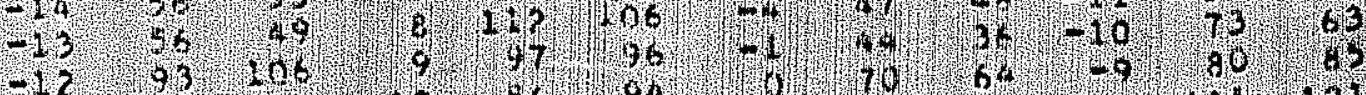

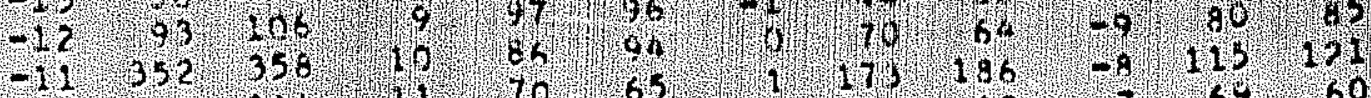

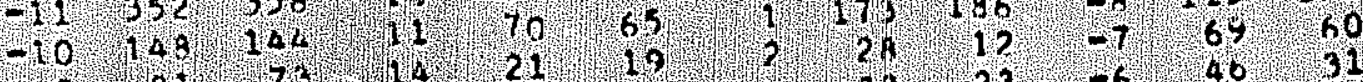

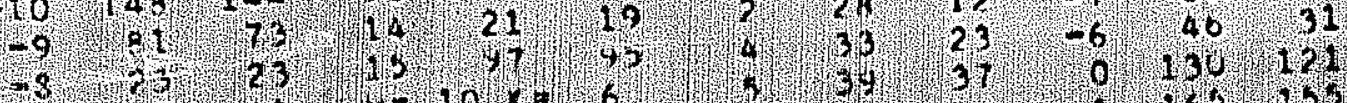

-7.

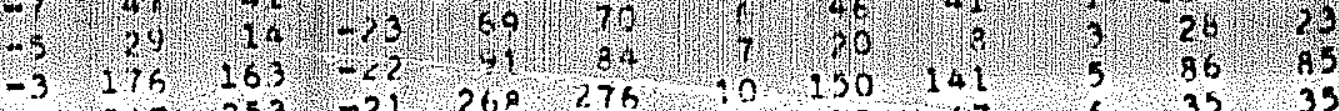

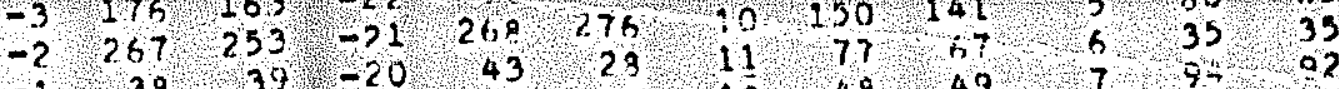

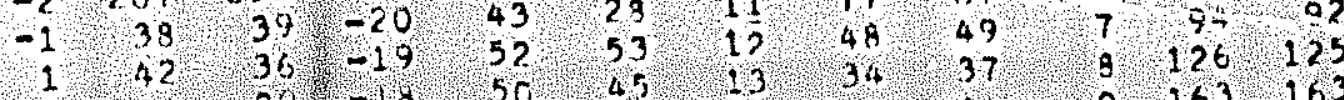

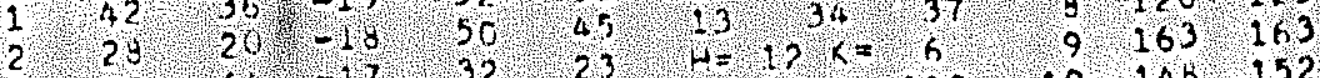

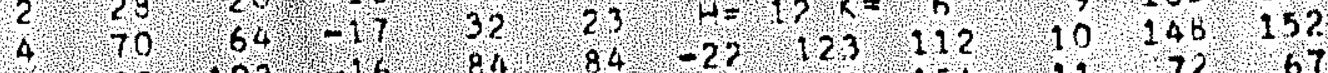

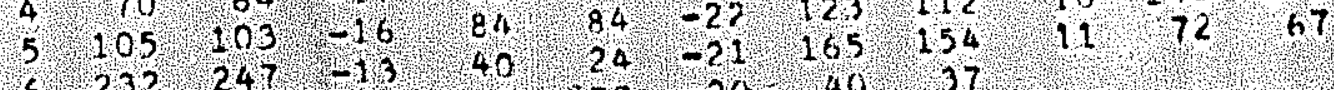

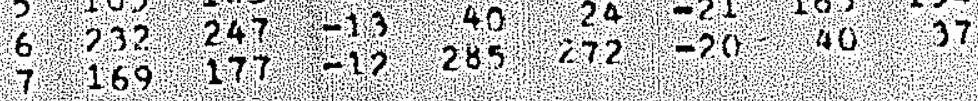




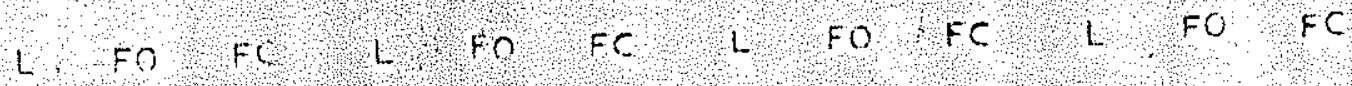

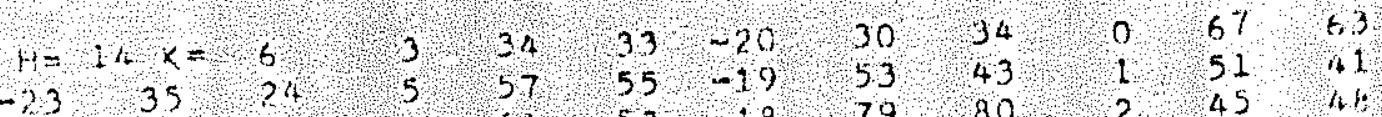

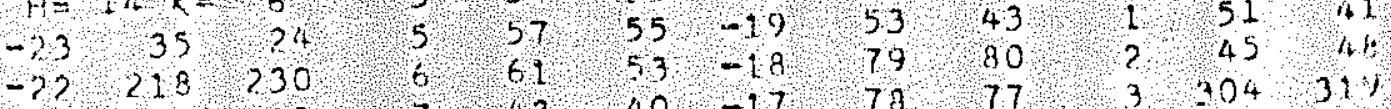

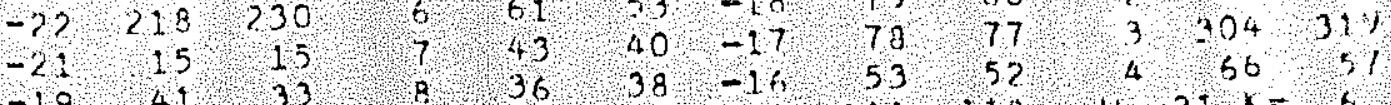

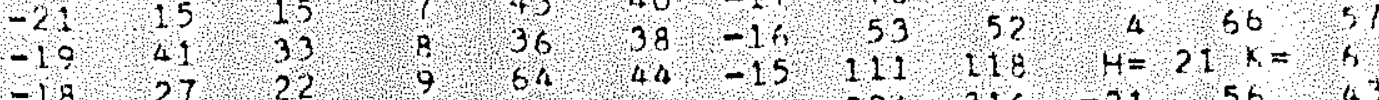

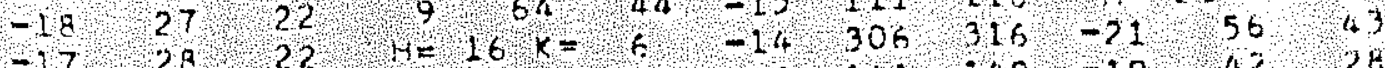

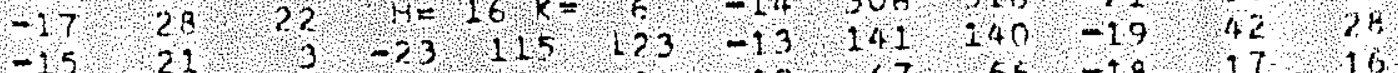

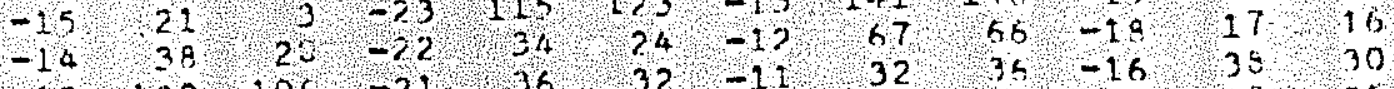

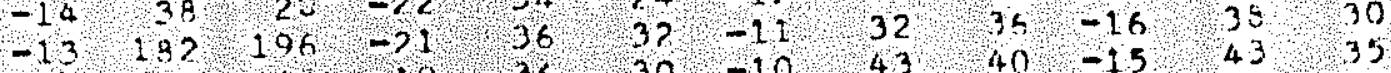

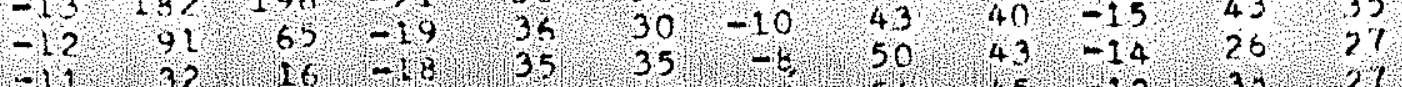

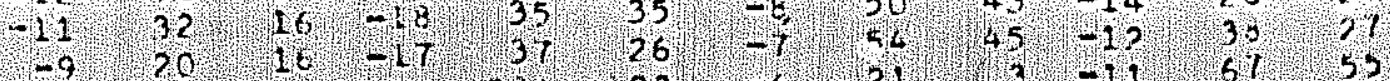

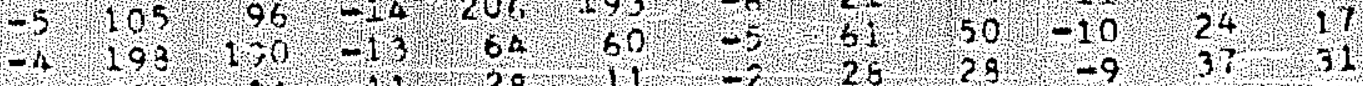

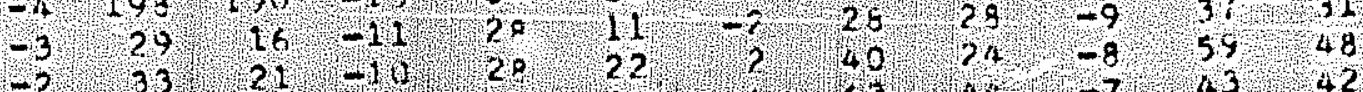

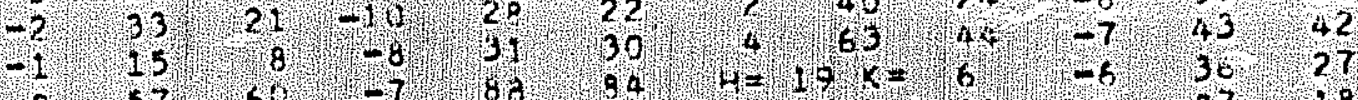

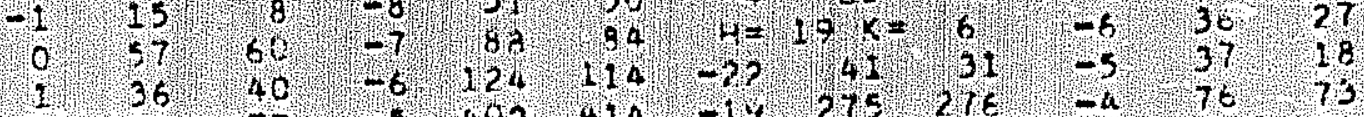

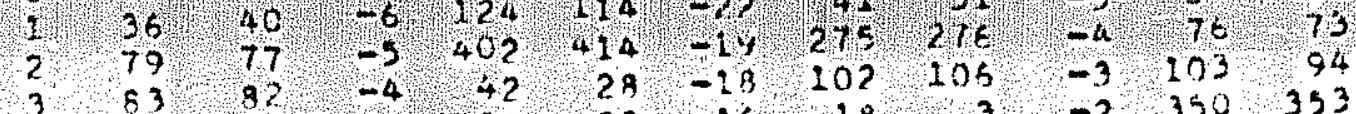

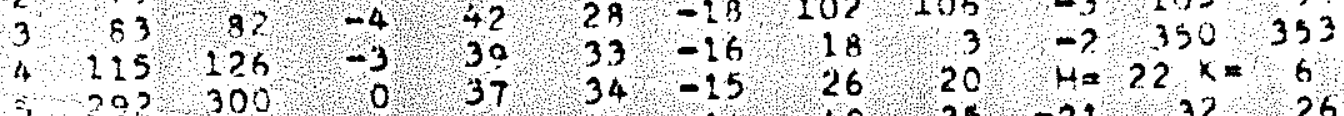

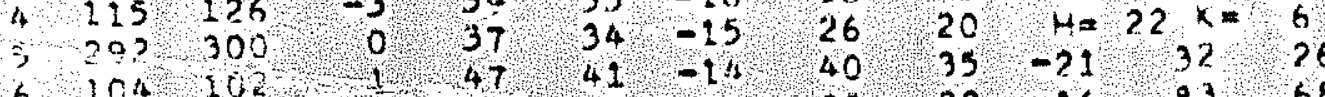

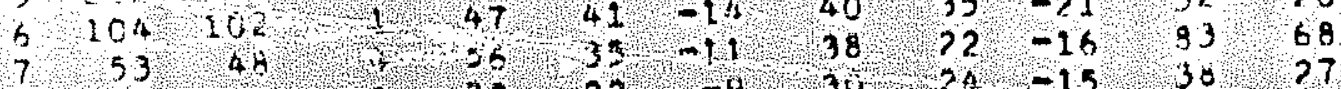

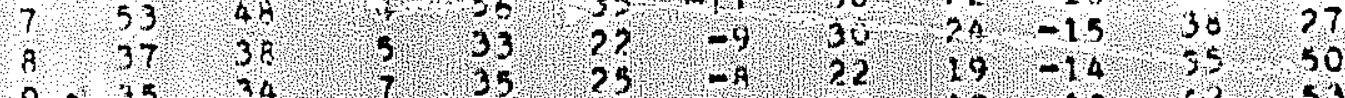

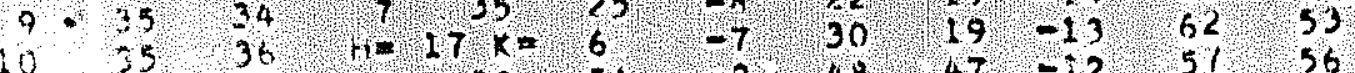

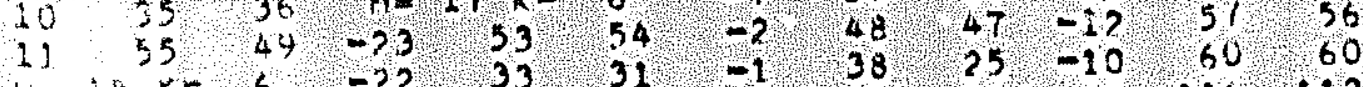

$\begin{array}{llllllllllll}-23 & 53 & 49 & -19 & 143 & 129 & 0 & 63 & 56 & -8 & 140 & 133\end{array}$

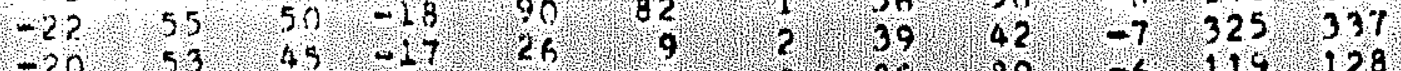

$\begin{array}{llllllllllll}-20 & 53 & 70 & -15 & 30 & 30 & 3 & 8 \% & 89 & -6 & 119 & 128\end{array}$

\begin{tabular}{llllllllll}
$-18 \mathrm{~d} 170,190$ & -14 & 51 & 44 & 4 & 47 & 47 & -5 & 73 & 69 \\
\hline
\end{tabular}

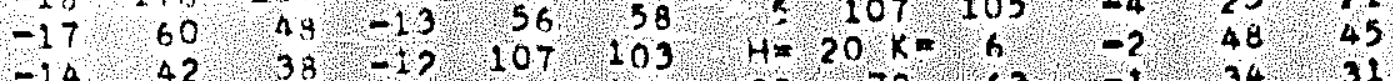

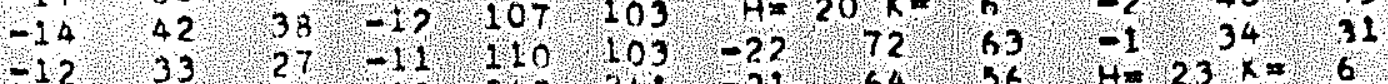

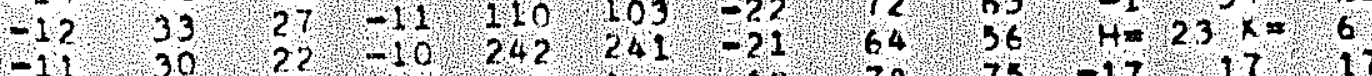

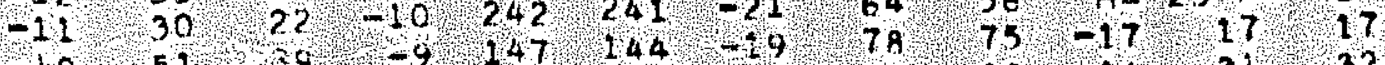

$\begin{array}{lllllllllllll}-10 & 51 & 69 & -9 & 147 & 10 & 10 & -17 & 28 & 22 & -16 & 31 & 32\end{array}$

$\begin{array}{llllllllllll}-9 & 204 & 194 & -7 & 10 & 63 & 56 & -16 & 59 & 48 & -15 & 54\end{array}$

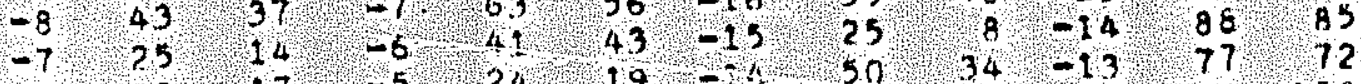

$\begin{array}{lllllllllllll}-6 & 30 & 17 & -5 & 24 & 19 & -1 & 50 & 34 & -13 & 158\end{array}$

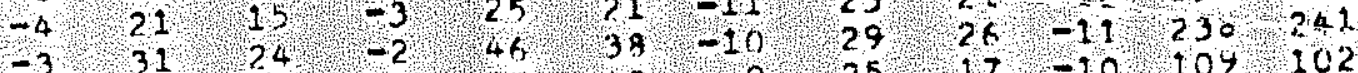

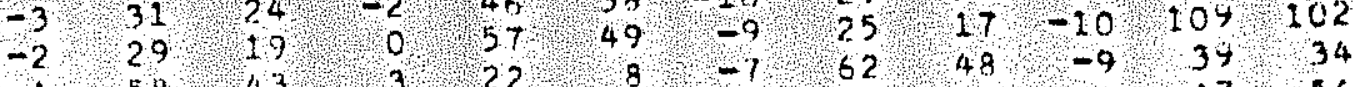

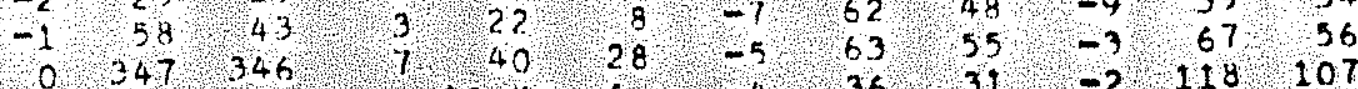

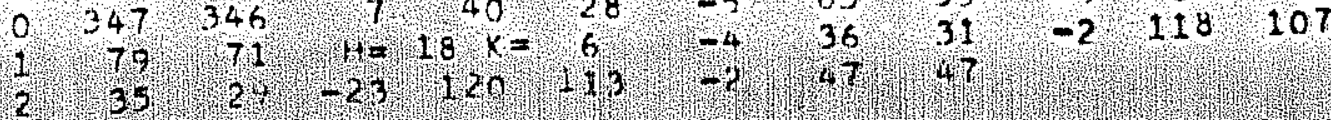




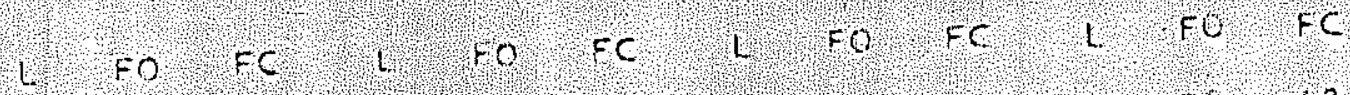

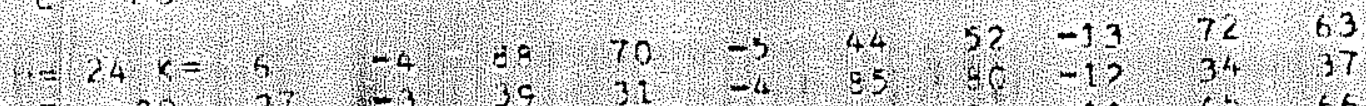

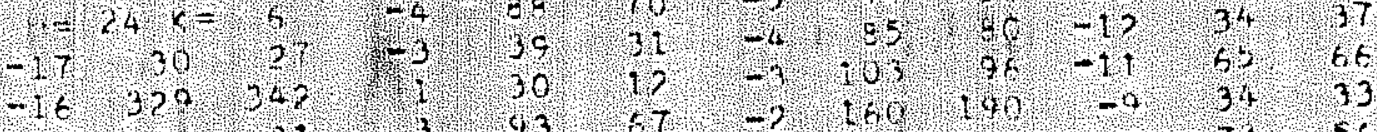

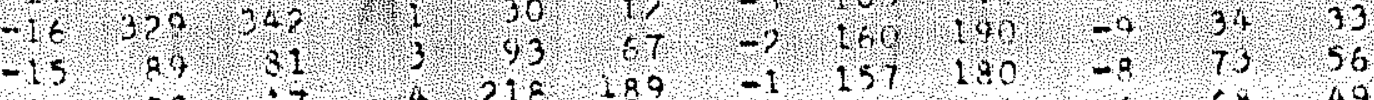

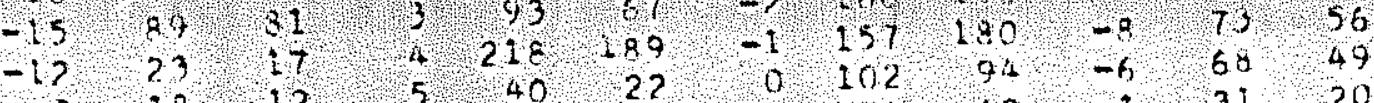

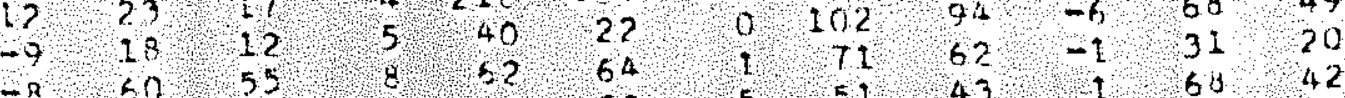

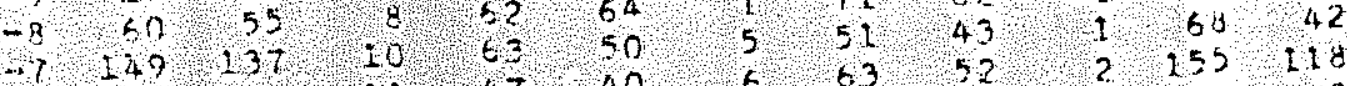

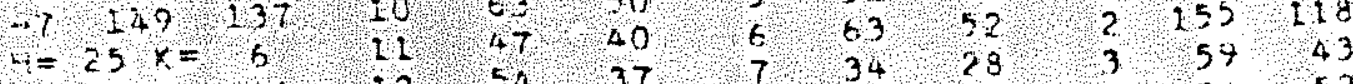

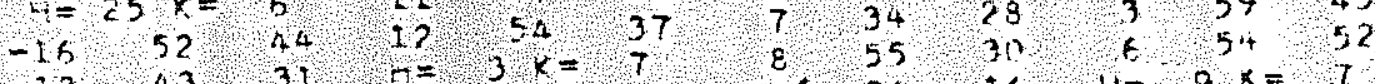

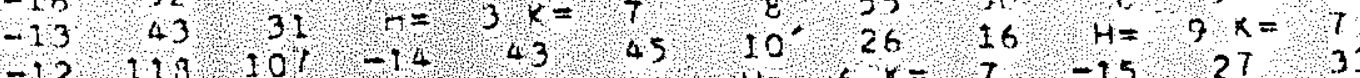

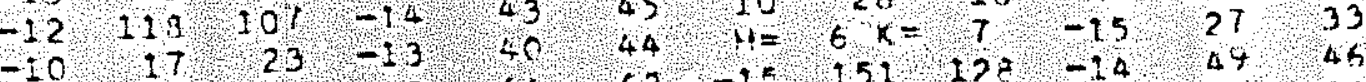

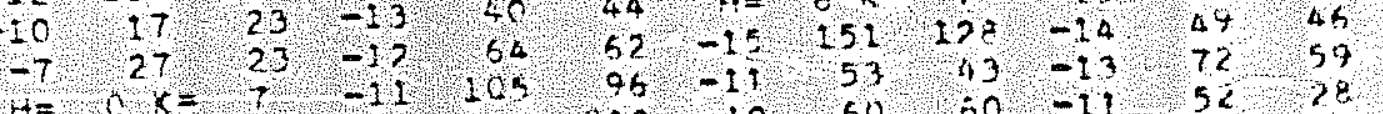

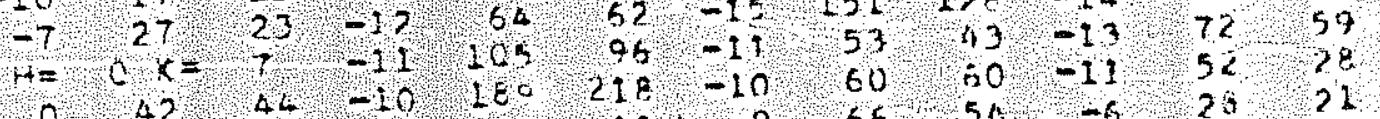

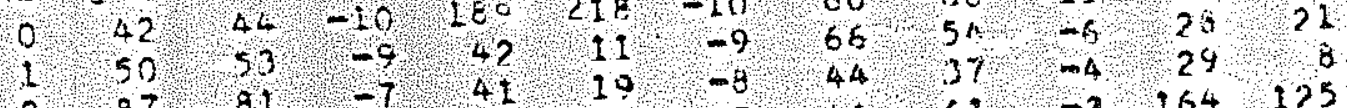

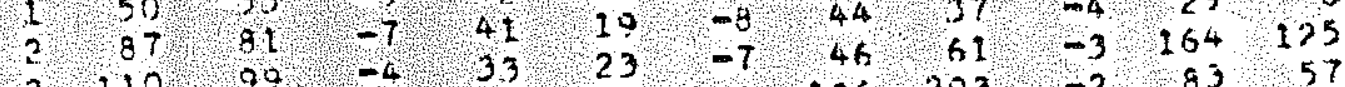

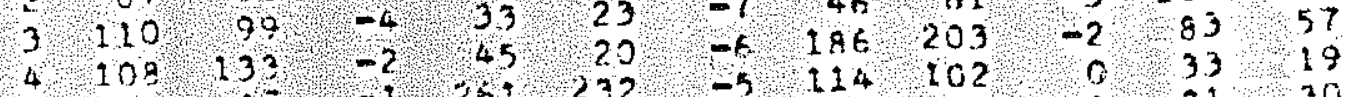

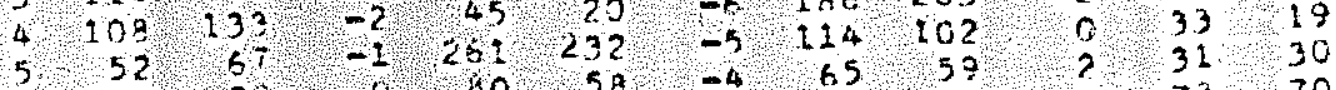

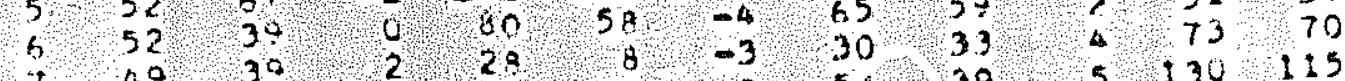

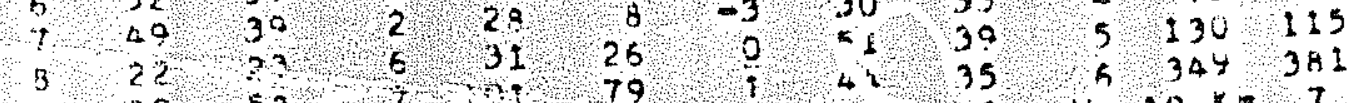

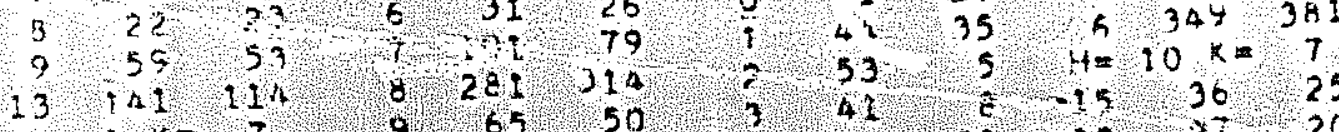

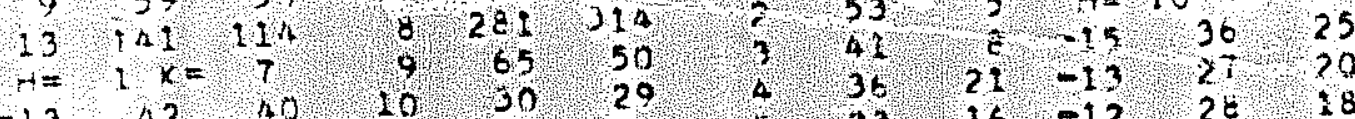

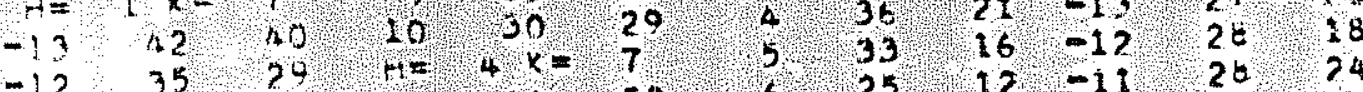

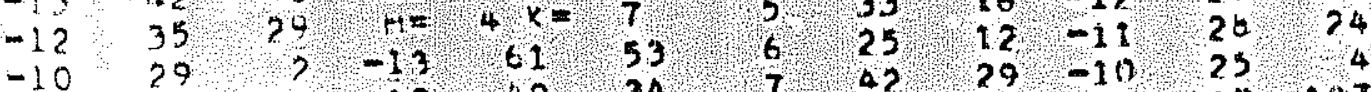

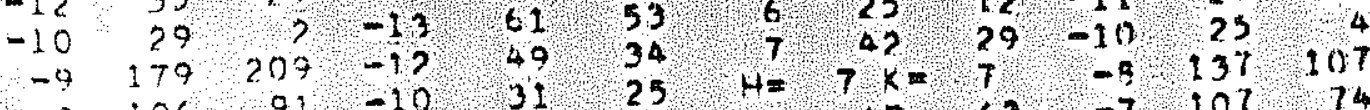

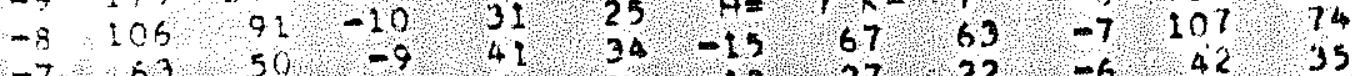

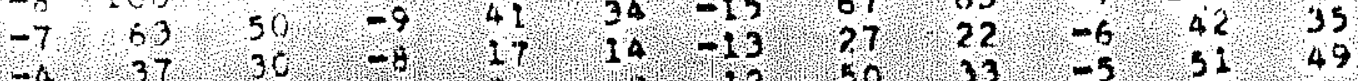

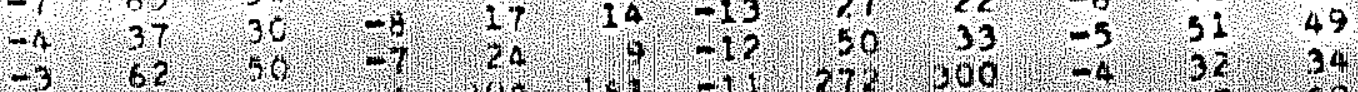

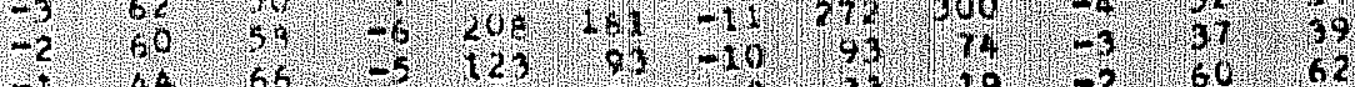

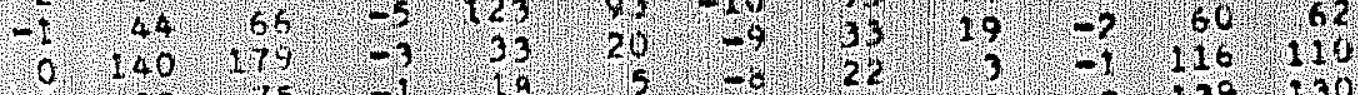

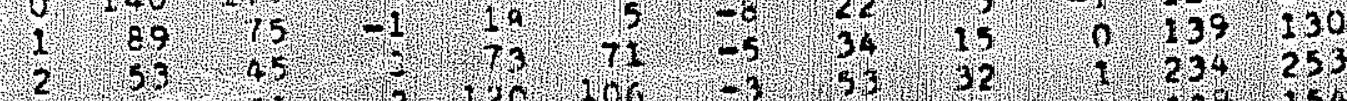

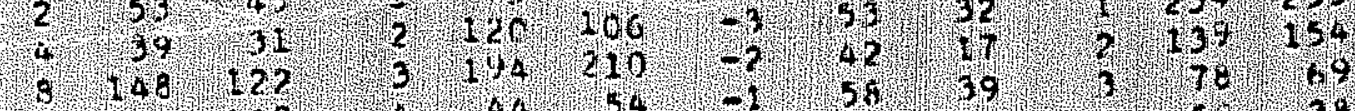

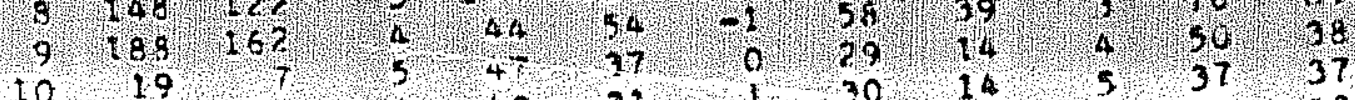

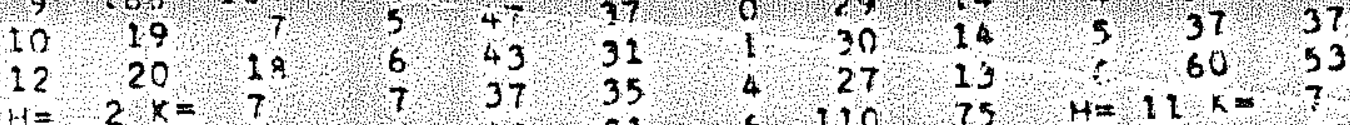

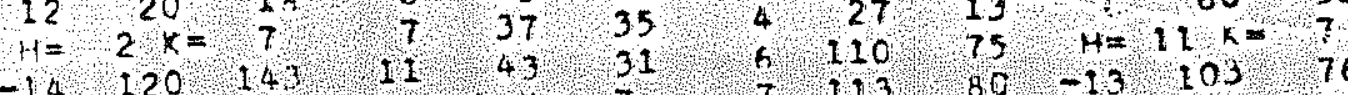

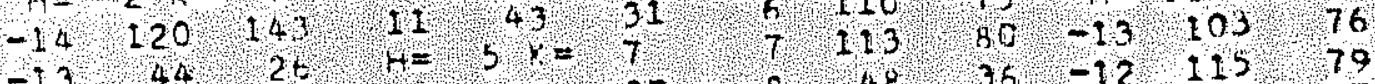

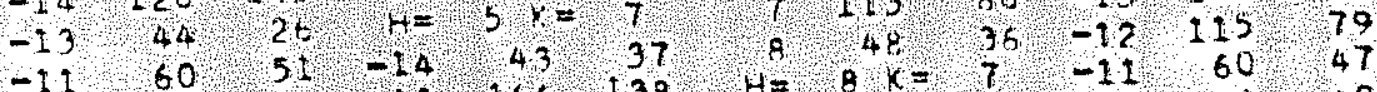

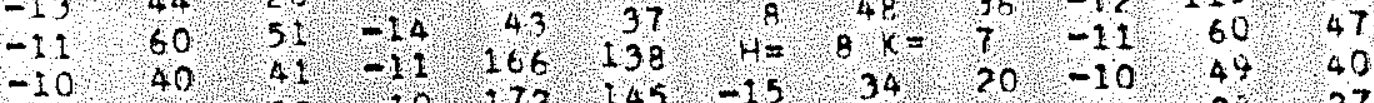

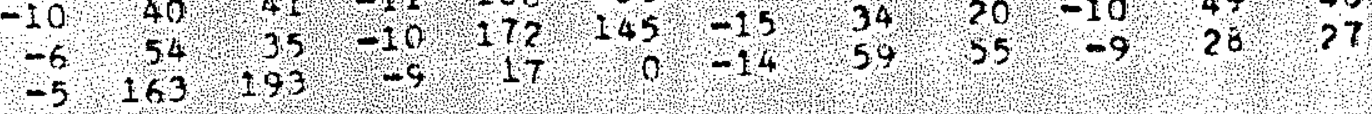




\begin{tabular}{|c|c|c|c|c|c|c|c|c|c|c|c|}
\hline$L$ & $F O$ & $F C$ & $L$ & Fo & $F C$ & $\mathrm{~L}$ & Fo & $F C$ & 1 & $F(j$ & $F C$ \\
\hline $\mathrm{H}=$ & $11 k=$ & 7 & -8 & 321 & 342 & -4 & 134 & 154 & -10 & 20 & 23 \\
\hline-7 & 50 & 65 & -7 & 85 & 171 & -3 & 77 & 51 & -8 & i7 & 13 \\
\hline-6 & 93 & 83 & -6 & 34 & 13 & -7 & 11 & 1 & -7 & 34 & 30 \\
\hline-5 & 89 & 82 & -1 & 25 & 10 & 1 & 29 & 25 & -6 & 38 & 8 \\
\hline-4 & 126 & 149 & -2 & 28 & 11 & 2 & 227 & 15 & -5 & $4 \%$ & 2 \\
\hline-3 & 301 & 333 & 9 & 69 & 94 & $4=$ & $1 / 4 x=$ & 7 & -3 & 50 & 45 \\
\hline-2 & 119 & 119 & 1 & $16 \pi$ & 131 & -13 & 468 & 72 & -2 & 48 & 34 \\
\hline-1 & 56 & 51 & 2 & ग 28 & 14 & -12 & 24 & 3 & $4=$ & $16 k=$ & 7 \\
\hline 1 & $\begin{array}{l}90 \\
34\end{array}$ & 26 & $1+=$ & $13 \times=$ & 7 \% & -10 & 41 & 28 & -10 & 451 & 38 \\
\hline$H=$ & $12^{34} x=$ & 21 & -14 & 96 & 97 & -9 & 125 & 94 & -9 & 11 & 11 \\
\hline-14 & ? ? & 22 & -13 & 929 & 350 & -8 & 116 & 92 & -9 & 80 & 70 \\
\hline-13 & 50 & 16 & -11 & 54 & 51. & -7 & 35 & 32 & -7 & 57 & 32 \\
\hline-12 & 44 & 41 & -10 & 53 & $\Delta$ & -0 & 3 & 3 & -6 & 41 & (2) \\
\hline-11 & 34 & 21 & -6 & 33 & (3) & -4 & 4 & 98 & -5 & 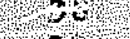 & 60 \\
\hline-10 & 19 & 12 & -6 & 21 & 1 & 10 & te & 35 & & 50 & \\
\hline-9 & 34 & 7 & -3 & 58 & 34 & $4=$ & $15 \mathrm{k}=$ & & & & \\
\hline
\end{tabular}

